\title{
Improving automatic processing of wildlife sound recordings
}

\author{
Julius Juodakis
}

\begin{abstract}
A thesis
submitted to the Victoria University of Wellington in fulfilment of the requirements for the degree of Doctor of Philosophy in Statistics
\end{abstract}

Victoria University of Wellington

Te Herenga Waka

School of Mathematics and Statistics

Te Kura Mātai Tatauranga 
Supervisors:

Prof. Stephen Marsland

Dr. Nirosha Priyadarshani 


\section{Abstract}

Acoustic monitoring of wildlife is emerging as a promising tool for animal conservation and research. Large amounts of natural sound recordings are routinely produced, but current use of this data is limited by lack of automatic analysis tools to process it efficiently. This thesis presents new methods for better detection of sound events, noise removal or robustness, and a framework for evaluating such improvements. Statistical and computational theory is used to support the generality of these tools, and also extended with results applicable outside of bioacoustics.

In the first study included in the thesis, we set out to establish a metric for bioacoustic population surveys that could be used to evaluate various design or analysis choices. Currently, a variety of metrics is used, such as the $F_{1}$ score or area under the curve for call detection. Using a combination of theoretical arguments and data examples, we show that rankings produced by these metrics depend on unobserved parameters, and do not necessarily correspond to overall survey performance in terms of ecological aims. These issues are avoided if the designs are ranked by the precision of spatial capture-recapture estimates. This framework covers a variety of practically important survey designs, and thus provides a single metric for general method evaluation.

The next study turns to the question of event detection. Both in acoustics and other fields, various problems feature series with temporary changes in a parameter. Estimating the onset and offset times of such events - changepoint detection - can be achieved in a principled and efficient way, but only if the background is stable, which is rare in practice. We build a two-type changepoint detector that is robust to nuisance dynamics, and demonstrate its use on different types of real data. The detector assumes that nuisance and signals events have different length, which allows distinguishing them even if the same parameter is affected. As part of this, we develop a faster algorithm for fixed-background changepoint detection, and analyse its properties in the case of changing mean of a Gaussian series.

The above changepoint detector is applied to acoustic events in the third paper of this thesis. We propose to combine it with a wavelet packet decomposition, thus producing a robust detector of frequency-specific energy increases. The theoretical analysis from Study II is extended to discuss the properties of this method. We 
test it on acoustic surveys using the evaluation framework developed in Study I, and observe consistently higher efficiency compared to other energy detectors. In a public challenge of household sound analysis, it was combined with a simple classifier to reach comparable performance to deep learning models with much less training data.

Study IV concerns wind and other transient broadband noises. They interfere with sound analysis, and their rapid dynamics counter most existing noise removal methods. We develop a short-term estimator of broadband noise level, based on polynomial models of wavelet packet spectrum. Two uses for the estimator are demonstrated: adjusting the detector from Study III to further reduce false alarms and improve survey efficiency, and restoring denoised sound by wavelet shrinkage. Various design choices are discussed, such as a robust alternative for noise estimation in rich soundscapes. 


\section{Acknowledgements}

This research was primarily supported by the New Zealand Marsden Fund, which is administered by the Royal Society of New Zealand Te Apārangi under grants 17-MAU-154 and 17-UOA-295. We also acknowledge funding from the National Science Challenge on Science for Technological Innovation; Victoria University of Wellington Doctoral Submission Scholarship; New Zealand Department of Conservation; Te Pūnaha Matatini, the New Zealand Centre of Research Excellence in Complex Systems; and MBIE Security for iconic species: Kiwi Rescue managed by Manaaki Whenua - Landcare Research.

The studies included in this thesis were made possible thanks to Zealandia, particularly Danielle Shanahan for allowing us to position recorders in the sanctuary, and Stephen Hartley, Paul Teal, and Heiko Wittmer for lending us acoustic recorders. We are grateful to Virginia Listanti, Alberto De Rosa, Rebecca Huistra, Emma Williams, Harry Caley, Tyler Jarman, and Alex White for their help in the field and in annotating the recordings. We also thank the reviewers and editors for their comments which helped improve the manuscripts during their production. 


\section{Contents}

1 Introduction $\quad 1$

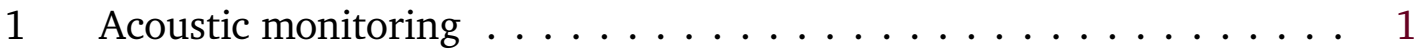

2 Bioacoustic data analysis workflows . . . . . . . . . . . . . 2

3 Recent developments in sound analysis methods . . . . . . . . . . . . 12

4 The need for statistically principled processing tools . . . . . . . . . 16

5 Avianz software . . . . . . . . . . . . . . . . . 17

6 Author contributions . . . . . . . . . . . . . . . . . . . . . . 19

2 Precision as a metric for acoustic survey design using occupancy or spa$\begin{array}{lr}\text { tial capture-recapture } & 29\end{array}$

1 Introduction . . . . . . . . . . . . . . . . . . . 30

2 Survey models and optimal design . . . . . . . . . . . . . . . 32

3 Simulation experiments . . . . . . . . . . . . . . . . 37

4 Kiwi survey methods . . . . . . . . . . . . . . . . . . . . . . . . 41

5 Kiwi survey results . . . . . . . . . . . . . . . . . . . 44

6 Discussion . . . . . . . . . . . . . . . . . . 47

7 Supplementary figures and tables $\ldots \ldots \ldots \ldots$

3 Epidemic changepoint detection in the presence of nuisance changes 61

1 Introduction . . . . . . . . . . . . . . . . . . . . . 62

2 Background ......................... 63

3 Detection of changepoints with unknown background level . . . . 66

4 Detecting changepoints with a nuisance process . . . . . . . . . . 69

5 Simulations . . . . . . . . . . . . . . . . . . 72

6 Real-world data . . . . . . . . . . . . . . . . . . . 76

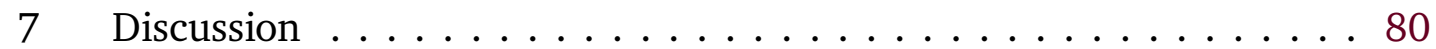

8 Supplementary material f . . . . . . . . . . . . . . . 84

4 A changepoint prefilter for sound event detection in long-term bioa$\begin{array}{lr}\text { coustic recordings } & 105\end{array}$ 1 Introduction . . . . . . . . . . . . . . . . . . . . 106 
2 Detection theory and new developments . . . . . . . . . . . . . 108

3 Experimental methods . . . . . . . . . . . . . . . . 113

4 Experimental results . . . . . . . . . . . . . . . . . . . . . 119

$5 \quad$ Discussion . . . . . . . . . . . . . . . . . . . . 122

6 Supplementary material . . . . . . . . . . . . . . . . 128

5 Wind-robust sound event detection and denoising for bioacoustics 145

1 Introduction . . . . . . . . . . . . . . . . . . . . . . . 146

2 Materials and methods . . . . . . . . . . . . . . . . . . . 148

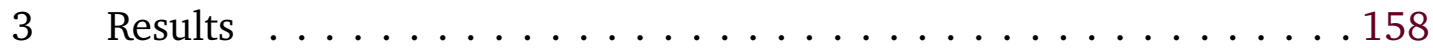

4 Discussion . . . . . . . . . . . . . . . . . . . 162

5 Supplementary material . . . . . . . . . . . . . . . . . 169

6 Conclusions $\quad 173$

1 Summary of the results . . . . . . . . . . . . . . 173

2 Future directions . . . . . . . . . . . . . . . . . . . . 174 


\section{Introduction}

\section{Acoustic monitoring}

Acoustic monitoring of wildlife is strikingly futuristic. Autonomous, programmable recorders are deployed in natural environments and collect sound data, which then provides insights into animal behaviour, ecology, and conservation management. Such projects can cover unprecedented spatial and temporal scales - such as the Australian Acoustic Observatory, planning to collect nearly 2000 years, or 2 petabytes, of sound data in total (Roe et al., 2021). Solar-powered, internetconnected networks of recorders in remote sites are becoming a reality, and still further developments are envisaged, including acoustic guides for poaching control (Wrege et al., 2017) or balloon-carried recorders for use in dangerous zones (Buehler et al., 2021). In New Zealand, where this thesis is written, bioacoustic monitoring is already routine, conducted in nation-wide projects and community groups, with locally developed recorders and analysis tools.

The acoustic monitoring data is recorded as a discrete-time sample of sound pressure - the waveform. A time-frequency visualisation of such data is shown in Figure 1. Various sounds and environmental noises are captured, as the recorders are not targeted, and have little shielding from wind or rain; in fact, interesting calls in practice are often rare and faint, unlike those seen in Figure 1. Such data is processed to detect, identify and describe the target sounds, remove noise, and fit ecological models. These practices could be informed by various statistical approaches for signal detection, estimation, and inference. (We note here that only single-channel data will be considered in this thesis. Multiple channel recording using synchronised microphones is also possible, and opens up more analysis options, such as cue localisation, but has rarely been available in bioacoustics so far (Rhinehart et al., 2020). The microphones are generally treated as omnidirectional, or nearly such, but in practice directional effects may be created by hardware or local microenvironment. Finally, our examples will mostly focus on terrestrial acoustics, although similar challenges are faced in marine bioacoustics.)

Yet, the processing of bioacoustic recordings is still often done either fully manually, or with large human involvement (Sugai et al., 2018; Kowarski and Moors- 


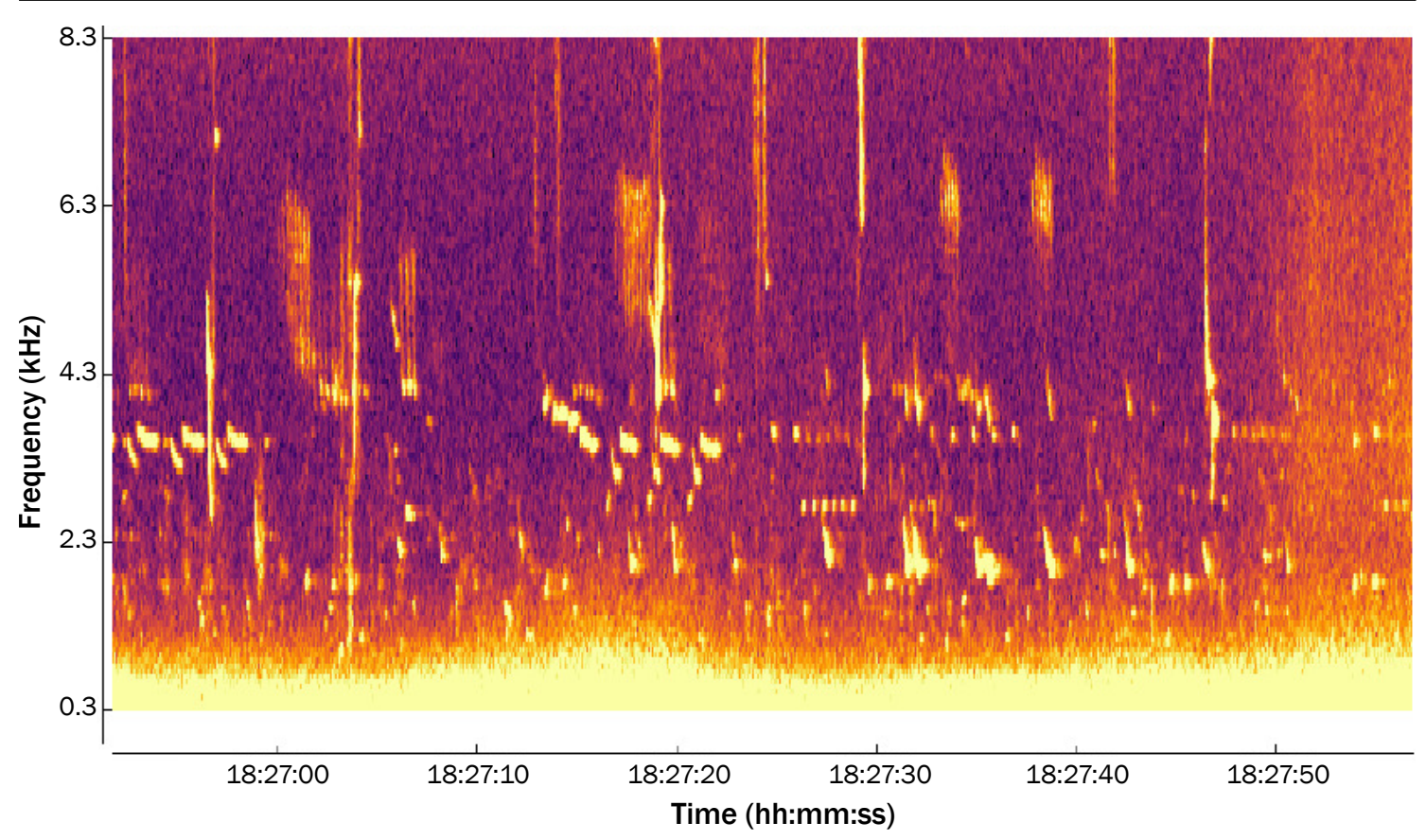

Figure 1: An evening at Zealandia: calls of various species are seen as bright shapes in this spectrogram, a time-frequency decomposition of a sound clip recorded in a wildlife reserve. Their detection, identification and separation from the environmental noise is the goal of this thesis.

Murphy, 2020). This is especially disappointing given the recent advances in commercial technologies for speech recognition, such as voice-controlled home assistants (Haeb-Umbach et al., 2019). The main goal of this work is thus to create statistically-principled methods that would allow automated, accurate and practically relevant analysis of bioacoustic data.

In the rest of this chapter, we will firstly review the inference models using bioacoustic data, as they determine the required data processing; then discuss some existing sound analysis methods and what limits their use in practice, and outline the approach that will be taken in the four studies comprising this thesis. The studies follow as chapters $2-5$. Chapter 6 will summarise the main conclusions, and provide some insights for further developments in this area.

\section{Bioacoustic data analysis workflows}

Sound recordings can be used to answer many different questions in ecology through various inference models (several example workflows are provided in Figure 2). We will review some of them, and specifically detail how their assumptions and requirements dictate the choice of methods for data processing. 


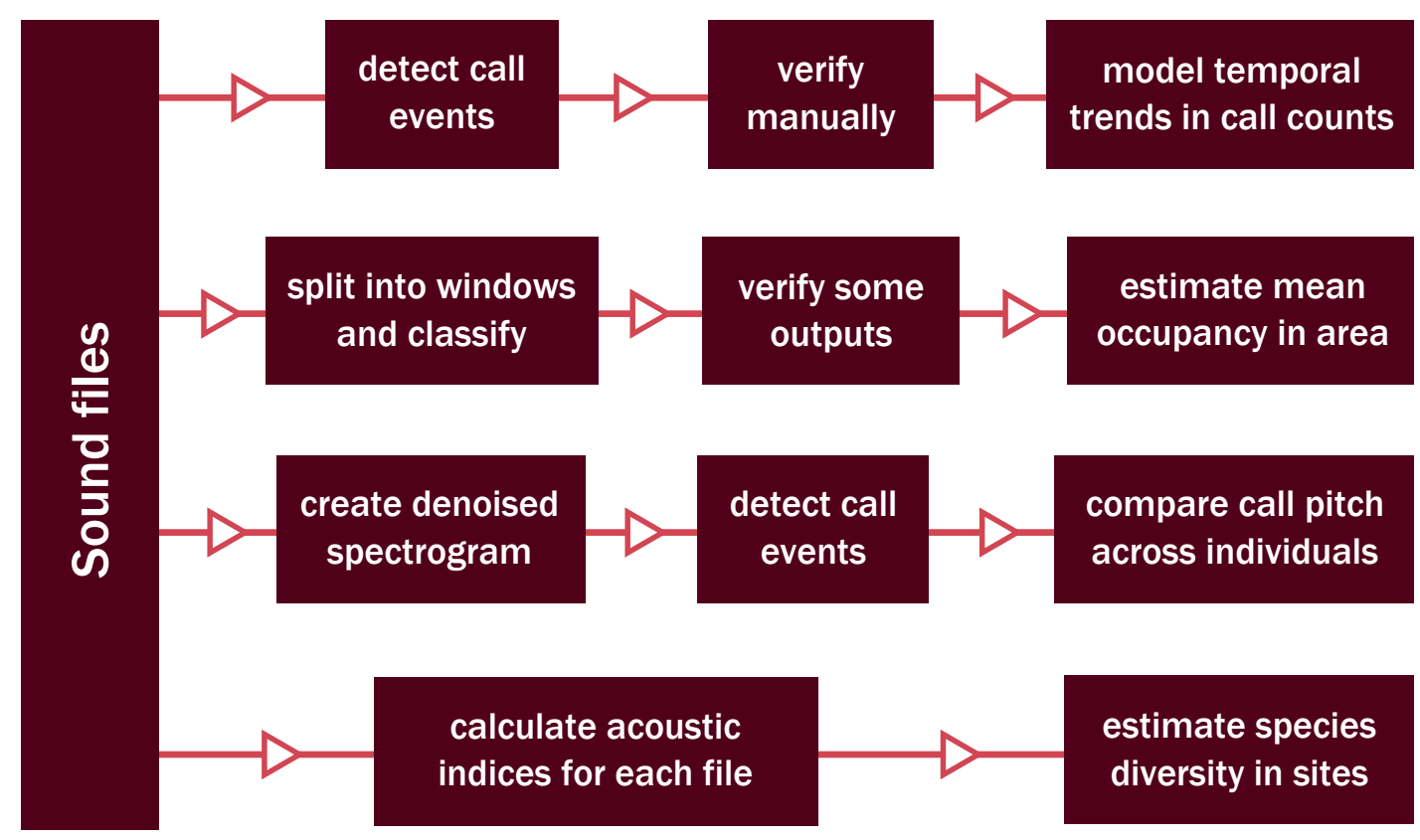

Figure 2: Some example schemes of data analysis workflows used in bioacoustics. The "windows" here are fixed-length segments covering the entire input, in contrast to event detection methods which estimate the start and end of each distinct event. Naturally, the appropriate input sound files may differ for each application.

\subsection{Inference based on call counts}

The simplest and most common way to use sound in monitoring is to count the number of cues associated with a target animal, and use that as a proxy for its abundance and activity. This proxy is tested for associations with various biological factors or interventions to identify behavioural responses, historic population trends, optimal conservation management, etc. Some examples among the many studies conducted in this framework are establishing bat activity at wind turbine height (Collins and Jones, 2009), measuring the effects of climate on cricket abundance (Jeliazkov et al., 2016), and evaluating bird response to urban light pollution (Watson et al., 2016). This is also the basis of 5 minute counts, a standard method of tracking New Zealand bird populations for the past 40 years (Dawson and Bull, 1975). Although originally conducted by experts in the field, such counts are increasingly often made from recordings, to allow replicability and more careful listening; either way, the inference framework is the same, and the number of calls (per time unit or a fixed-duration survey) remains the main analysis outcome.

A well known issue with the basic counting framework is that the number of cues captured in a recording or a field survey, where by captured we mean present at sufficient clarity for an expert to identify, depends not only on the number of 
animals in the area, but also their vocal behaviour and various factors influencing detection. In particular, environmental noise such as wind or rain greatly reduces the ability to detect target sounds, both by humans and by automatic methods (Priyadarshani et al., 2018a,b). Thus, comparing call counts obtained in different weather conditions is difficult, and more generally the inference of spatial or temporal trends will be confounded by environmental factors. One approach to solve this is by including various such factors as covariates in the (typically) generalised linear models used for inference, as in e.g. Digby (2013). However, relevant covariates for each species are typically unknown or unmeasured in historic data, the adjustment greatly increases the amount of data required, and even then does not allow identifying the true impact of the adjustment factors on the animal behaviour, which may be of interest.

Therefore, methods were devised that estimate detection probabilities from the data, at survey or even finer level. This is achieved by introducing a more formal description of the cue emission and observation processes. For example, in distance sampling, the distances from observer to each calling animal are recorded. The animal locations are assumed to be uniform with a density $D$ around the observer, and the probability to detect a call emitted at distance $x$ is modelled with a parametric function $g(x)$, for example, the half-normal:

$$
g(x)=\exp \left(\frac{-x^{2}}{2 \sigma^{2}}\right)
$$

The survey-level detection strength is thus represented by a single parameter $\sigma$, estimated by fitting $g(x)$ to the observed distance distribution. More generally, we can define this model by the likelihood $\mathscr{L}(n, \mathbf{x})$ of detecting $n$ cues at distances $\mathbf{x}=\left[x_{1}, \ldots, x_{n}\right]$ (Buckland et al., 2015):

$$
\mathscr{L}(n, \mathbf{x})=P(n) \prod_{i=1}^{n} f\left(x_{i}\right)=P(n) \prod_{i=1}^{n} \frac{g\left(x_{i}\right) D\left(x_{i}\right)}{P_{d}}
$$

where $P(n)$ is the probability of observing $n$ cues, based on a binomial or Poisson, $f$ is the pdf of distances, $D(x)$ is the true density of calls at $x$, and $P_{d}$ a normalising constant. This form can be readily extended to incorporate different sampling designs, known measurement errors, other types of recorded information etc., simply by choosing appropriate parametric models for the observations and adding those to the likelihood. (The full-likelihood form is presented here to emphasize this potential generalizability; typically in distance sampling the $P(n)$ component 
is conditioned out, and the density is estimated using the fitted $g(x)$ and design considerations.) The most general formulation of this framework is known as spatially explicit capture-recapture, SECR or SCR (Borchers et al., 2015; Dawson and Efford, 2009). Figure 3 shows an example of acoustic data analysis in this framework. We use it throughout this thesis, and provide a more detailed introduction in Chapter 2. The distance sampling-SCR connection is further presented in Buckland et al. (2015), and some other variations on this theme are reviewed in Dénes et al. (2015). In all these models, the main outcome is the density $D$ (or spatially varying $D(x)$ ), which can be used as a detection-adjusted replacement for the call counts in any ecological inference.

The summary above neglects an important distinction between animal and call counts. The main unit of all the models discussed so far is a call; converting their outputs into densities of animals, which are more ecologically relevant, requires either a known and constant call rate, or unsupervised assignment of calls to individuals, or a model relating animal movement to calling behavior. These solutions are discussed further in Stevenson et al. (2015, 2021), and many studies on individual identification, e.g. Cheng et al. (2010). Nonetheless, this issue is not specific to the count methods, and all acoustic measures are currently treated as relative indices of abundance.

Implications for the analysis workflow. As the models presented in this section rely on cue counts, the goal of sound processing is event detection - identifying the start and end times of each target call. (The terms segmentation, pre-filtering and event detection are used almost interchangeably in the literature, and will be used so in this thesis.) Missing a number of true calls during this process is not critical, as it is equivalent to reduced detection ability, which is estimated by the model anyway (Dénes et al., 2015). In contrast, false positives - for example, misclassifications of a similar species as the target - are not included in the model and may affect the estimation unpredictably. Combined with the sparsity of target calls in typical conservation settings, this suggests that a reasonable pipeline would consist of automatic segmentation and manual vetting (see Figure 2): automatic methods can effectively identify most non-target periods and mark putative segments, but expert review is currently still necessary to avoid false detections (Marsland et al., 2019; Buehler et al., 2021). The automatic tools then may involve denoising, peak detection and smoothing, segment classification or other stages, as appropriate to create an output that is suited for human verification.

The count models also enforce more specific requirements on the design of pro- 


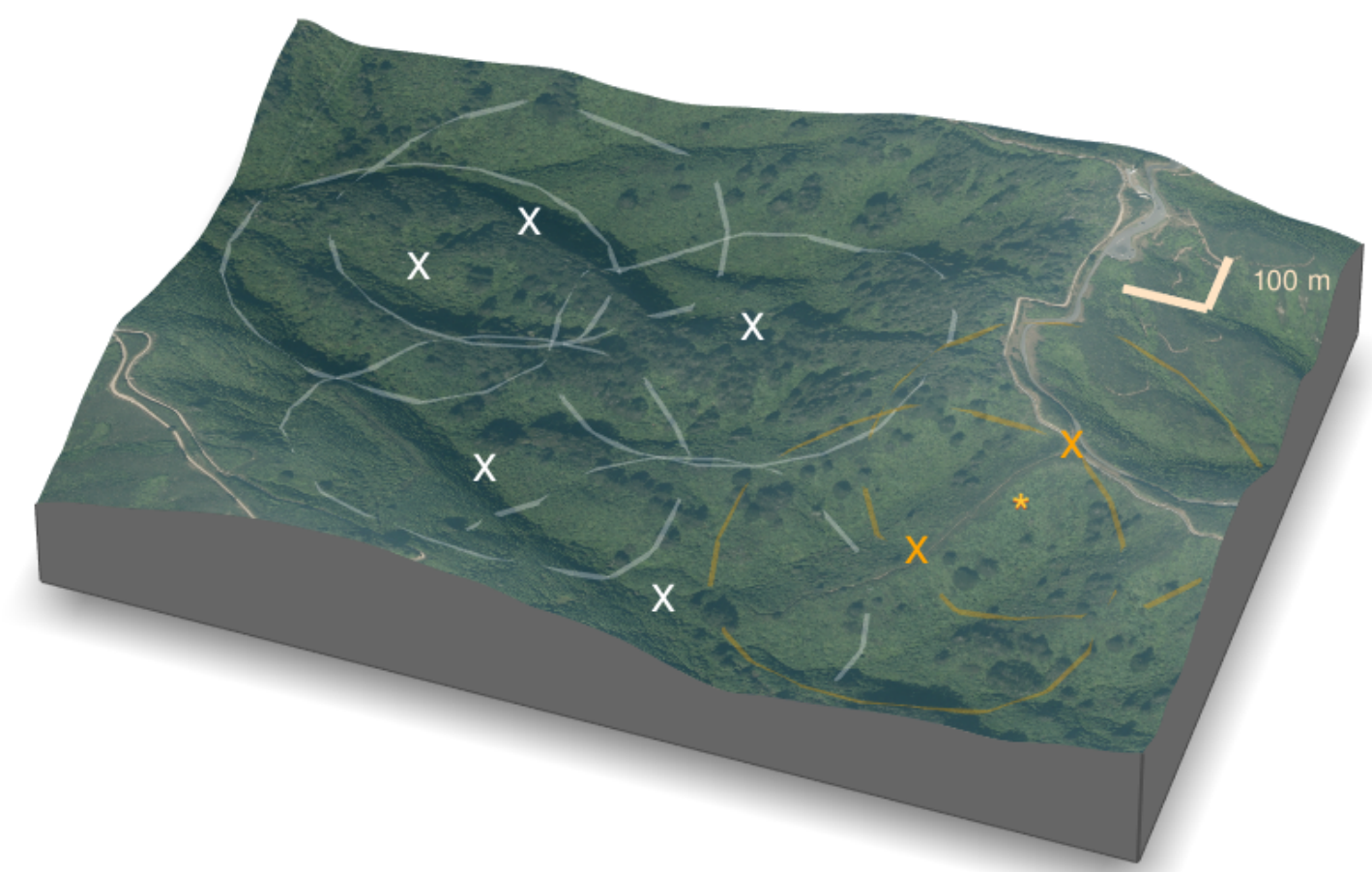

Figure 3: Illustration of acoustic survey modelling by SCR. X marks locations of 7 recorders in Zealandia sanctuary, used in this thesis. Based on capture histories of 335 kiwi calls, a call density and a detection function were fitted; the circles around each recorder show the radius at which the estimated detection probability is $50 \%$. An example call was detected on the two detectors highlighted in orange - from that, its most likely location can be estimated (marked with asterisk).

cessing tools. Ensuring that detection of each call is independent and with probability that monotonically decreases with distance is important, and can be easily overlooked. An instructive case is presented in Oedekoven et al. (2021), where observers inspected spectrograms from 7 recorders simultaneously, and therefore were likely to provide the same decision on all, resulting in 15-fold overestimated detection distances for SCR. Examples of automatic detectors that violate this condition are presented in Knight and Bayne (2018). The call detection must also be precise in time, because these models estimate the detection parameters using recaptures of a call across multiple recorders, or features extracted from the segments such as received sound pressure (Borchers et al., 2015). Notably, the very definition of a call is adjusted based on the inference framework. For example, if the cue locations are modelled as independent, repeated sequences from a single individual can be defined as a single "call", either by adjusting the detection stage or manually afterwards (Dawson and Efford, 2009). 


\subsection{Inference based on presence-absence}

Another group is comprised of studies that only record presence or absence of a target species calls in multiple sites. Like call counts, these measures are often directly used as a proxy for animal activity, to investigate associations with spatial or temporal covariates, e.g. Risch et al. (2019); Charish et al. (2021). And like call counts, these measures are made incomparable by varying detection probabilities, and also underestimate the true presence because of imperfect detection. To address these issues, the occupancy model was developed (MacKenzie et al., 2002). These models estimate the detection parameters by using repeated visits (occasions), which in acoustics are typically obtained by splitting the monitoring period into 10-30 min clips (Kalan et al., 2015; Metcalf et al., 2019; Doser et al., 2021). At each site $i$ on visit $t$, presence or absence is recorded as $x_{i, t}$. The detection then is modelled hierarchically:

$$
\begin{aligned}
y_{i} & \sim \operatorname{Bernoulli}(\Psi) \\
x_{i, t} \mid y_{i} & \sim \operatorname{Bernoulli}\left(y_{i} p\right)
\end{aligned}
$$

Here $y_{i}$ is the actual state of species in the site, $p$ is the detection probability given presence, and $\Psi$ is the occupancy. The latter is analogous to the density $D$ in count models, in that it is the main target of estimation and is used to test ecological hypothesis. Some examples are identifying habitats preferred by koalas (Law et al., 2018) or monitoring occupancy changes with dynamic models that include colonisation and extinction (Metcalf et al., 2019). The occupancy estimates, or the naïve presence-absence, can also be combined into species richness measures (Smith et al., 2020; Steel et al., 2019), which are then used in inference.

The site-level occupancy model can be integrated with more detailed information about the observed calls. If, instead of simple presence-absence, $x_{i, t}$ is the call count, then (1) is replaced with:

$$
\begin{aligned}
y_{i} & \sim \operatorname{Bernoulli}(\Psi) \\
x_{i, t} \mid y_{i} & =\left\{\begin{array}{l}
0, \text { if } y_{i}=0 \\
Z \sim \text { zero-truncated Poisson }(\lambda), \text { if } y_{i}=1
\end{array}\right.
\end{aligned}
$$

Note that a parameter $\lambda$ was introduced, which captures the number of animals in site, their call rate and the ability to detect them. This framework is used to explicitly model misclassifications in Wright et al. (2019). Another variant built on 
(2) is time-to-detection analysis, where this information can be used to increase occupancy estimate precision, or to allow modelling from single visit data (Whittington et al., 2019). Overall, occupancy models are popular, and a large literature is devoted to their analysis and extensions.

Implications for the analysis workflow. Models based strictly on presenceabsence allow considerable freedom in the analysis methods. Since precise timestamps are not important, it is possible to use detection-by-classification directly, i.e. to classify short windows of recording as target present/absent, without attempting to split or join them into distinct calls. Applying an "OR" operation over these outputs then produces a presence/absence decision for each visit. Indeed, it is tempting to use long input windows, closer to the duration of a visit, and analyse features summarised over the scale of a minute or more, such as the "long-duration spectrograms" (Towsey et al., 2018; Znidersic et al., 2020). Standard short-window detectors can also appear to perform better when evaluated over longer periods: Metcalf et al. (2019) presented detectors that can accurately determine bird presence/absence in each 15 min visit, even though they capture only 8-10\% of individual calls. However, this calls into question what such detectors are actually capturing, and also development and tuning of detectors becomes more complicated if visit-level properties such as temporal call clustering must be taken into account. Because of these reasons, sound analysis for presence-absence models still mostly uses standard detection workflow aimed at the call level.

Naturally, for procedures that need call-level annotations (Wright et al., 2019; Whittington et al., 2019), the workflow becomes similar to that presented for count models earlier.

There are also fewer requirements for the detection performance in the presenceabsence framework. Because the detection process is not modelled in a spatially explicit way, only with a site- or visit-specifc probability, strict decay with distance is not assumed. Importantly, occupancy models can allow false positives, if some of the sites are surveyed with a more certain method that does not produce them (it can still miss some true calls; Clement et al. (2014)). This is particularly applicable to the manual verification workflow, as then automatic detections can be reviewed for some sites, providing the "certain" reference, and used directly for others, saving expert time. Such workflows are actively studied in the occupancy literature: for example, Kalan et al. (2015) and Rempel et al. (2019) discussed optimal review effort allocation, and Doser et al. (2021) further extended the workflow to integrate field count data. In theory, manual review could be entirely bypassed if 
the detector's false positive rate is known. However, this rate entirely depends on what other similar species are present in the area, and so cannot be directly determined from external data. This can potentially be solved by using a more detailed model of counts and misclassification rate for all observed species (Wright et al., 2019).

It should be noted here that even expert identification of animal calls is far from certain (Clement et al., 2014; Mortimer and Greene, 2017). An interesting, but labor-intensive way of estimating misdetection rates with multiple reviewers is proposed in Rempel et al. (2019). In this thesis, we focus on species which are clearly identifiable from calls, and in particularly ambiguous cases choose to err on the side of false negatives in our annotations, so our positive annotations can be assumed to be largely correct.

\subsection{Extraction of call features}

Not only changes in call abundance can be relevant in ecology, but also changes in call qualities. The acoustic niche hypothesis states that vocalising species in a community will adapt their calls to avoid overlap (Ruppé et al., 2015; Schmidt et al., 2015). Similarly, increasing loudness, pitch or signal duration could be helpful for animals communicating in noisy environments (Wrege et al., 2017; Dunlop et al., 2014). To test these and other hypotheses about behaviour, features such as frequency bandwidth, call duration, number of sub-call elements, loudness, dominant or fundamental frequency, or others crafted specifically for the target sound, must be extracted from a large number of calls.

The goal of processing for these tasks is to estimate the call timestamps, and the features can then be calculated with several standard tools. This is similar to the call count workflow, but here the estimation of event times must be even more precise, as any captured non-target period may impact the measurements. The methods may be required to operate on short time windows as well, with features sometimes defined on the scale of $0.001 \mathrm{~s}$ (Schmidt et al., 2015). In addition to the timestamp, precise feature calculation may require a more detailed contour of each event in the time-frequency plane, or ideally full extraction from other signals, to prevent interference. This problem is known as blind source separation (Lin and Tsao, 2019); however, with single-channel data, it is ill-posed, and requires further assumptions about the possible signals to identify a solution. Denoising is also more important with these tasks, and introduced as a separated stage before the feature extraction (Mallawaarachchi et al., 2008). The main constraint in this workflow is 
that the original sound features must be faithfully preserved, which is not easy to ensure with more complex methods. All these methods may be computationally intense, so a basic pre-filtering followed by more precise analysis is a likely approach for this type of studies.

Call feature extraction is tightly linked to other inference types. The features are often used as input in automatic classifiers, for distinguishing species, call types or individuals, with the aim to use the results in density or occupancy models (Xie et al., 2018; Digby, 2013; Hahn et al., 2013). One reason to choose this workflow is that it reduces the dimensionality, compared to recognition directly from a spectrogram or waveform. Secondly, analysis based on parametric features may be more explainable, and in turn lead to mechanistic insights about animal perception. In this usage, precise detection and denoising are still important for accurate feature extraction, and the standard assumptions of the inference model must also be met, as described earlier.

\subsection{Real-time monitoring}

An emerging application for acoustics is real-time wildlife monitoring. Examples proposed so far include: adjusting maritime activities to avoid whales (Baumgartner et al., 2019), automatic warning or repellance systems for elephants approaching settlements and highways (Zeppelzauer et al., 2014; Wrege et al., 2017; Dampage et al., 2021), coordinating fast response to poaching (Kalmár et al., 2019; Wrege et al., 2017), observing farm animal behaviour (Chelotti et al., 2016), and real-time monitoring of ecological impacts during disaster response (Kobayashi et al., 2018). The distinguishing feature of this type of applications is that the sound analysis must be entirely automated, without involving human review. The computation complexity of detectors also becomes a major concern, since the processing is typically done by the recording device. Creating accurate sound event detectors within these constraints is difficult, and such applications are not yet widely employed; better data analysis algorithms, increasing connectivity in remote locations and more energy-efficient hardware can make these applications practical in the near future.

\subsection{Ecosystem evaluation by indices}

An alternative framework of ecological inference is provided by ecoacoustic indices. They are summary statistics, calculated directly from a time-frequency represen- 
tation of a recording, without segmenting it into calls. For example, the acoustic complexity index (ACI; Farina et al. (2011)) is the total variation of spectrogram intensities over a chosen window of size $\Delta f \times \Delta t$ :

$$
A C I_{f, t}=\sum_{i=f}^{f+\Delta f} \frac{\sum_{j=t}^{t+\Delta t}\left|I_{i, j}-I_{i, j-1}\right|}{\sum_{j=t}^{t+\Delta t} I_{i, j}}
$$

where $I_{i, j}$ is the magnitude of a spectrogram at row $i$ and column $j$. Another popular index is the normalised difference soundscape index (Kasten et al., 2012), which is simply the ratio of energies in two frequency bands $\left[f_{1}, f_{2}\right],\left[f_{3}, f_{4}\right]$, approximately chosen to represent "natural" and "anthropogenic" sounds:

$$
N D S I_{t}=\frac{B-A}{B+A} \text {, with } B=\sum_{i=f_{1}}^{f_{2}} I_{i, t}^{2}, A=\sum_{i=f_{3}}^{f_{4}} I_{i, t}^{2} \text {. }
$$

Several other indices are built on Shannon's entropy, applied to the distribution of spectrogram intensities (Eldridge et al., 2018). These and other index series can be further summarised over sites or longer periods by in turn calculating their own summary statistics or composite indices (Farina et al., 2011; Eldridge et al., 2018; Roca and Opzeeland, 2019).

Such indices are popular, as they provide a simple way to measure species diversity, avoiding the laborious call detection process (Bradfer-Lawrence et al., 2020), but also used in their own right for holistic description and classification of sound environments (Roca and Opzeeland, 2019). However, it is unclear how well they actually measure ecologically relevant properties. For some of the major indices, including ACI, it is currently not even established whether they should increase or decrease with species richness; studies of Mammides et al. (2017), Metcalf et al. (2020), Shamon et al. (2021) find associations in opposite directions to those reported in Eldridge et al. (2018) and Bradfer-Lawrence et al. (2020). Major confounding by site properties and time of day is observed, drastically changing the strength of the association or even reversing its direction within a single study (Eldridge et al., 2018; Metcalf et al., 2020). Possibly, intermediate-complexity frameworks could be developed in the future, that would allow partial separation of noise and so reduce confounding compared to indices, but still require less effort than complete detection of multiple species at the call level. 


\section{Recent developments in sound analysis methods}

\subsection{Machine learning literature}

Bioacoustic data analysis, primarily methods for sound event detection, is a major topic in the field of machine learning. Besides a huge number of publications, two series of public competitions are active: BirdCLEF 2014-2021 (www . imageclef . org) is primarily focused on bird sound detection, while DCASE 2016-2021 (www . dcase.community) is a general sound analysis competition which also features bioacoustic tasks. These challenges present a forum for standardised testing and reviewing the state-of-the-art methods every year.

Currently, the de facto standard in sound detection research is spectrogram classification by convolutional neural networks (CNNs), as seen in e.g. public competitions (Serizel et al., 2018). The spectrogram is a time-frequency representation of a sound recording (denoted here $x$ ), based on the discrete short-term Fourier transform:

$$
I_{f, t}=\left|S T F T_{x}(f, t)\right|^{2}=\left|\sum_{\tau=0}^{N-1} x(\tau) w(\tau-t H) e^{-i 2 \pi f \tau / L}\right|^{2}, \text { for } f \in 0, \ldots, L / 2
$$

here parameters $H, L$ (the hop and length) determine the window of samples used to calculate the transform, and $w$ is a windowing function, typically smoothly decaying away from 0 . The spectrogram intensities can be transformed in various ways before usage, such as changing the scale (Knight et al., 2019), creating color channels as a third dimension by mapping different parts of the scale to each (Dennis et al., 2011), or passing the spectrogram through a Mel filterbank, which in effect combines the nearby frequencies into larger bands (Cheng et al., 2010).

To detect sound, a slice of spectrogram $\left(I_{f, t}\right)_{f \leq L / 2, t_{1} \leq t<t_{2}}$, or an equivalent transformed representation, is provided as input to a CNN (Figure 4). The network applies successive layers of operations such as convolution with small 2D kernels (typically $3 \times 3$ units), pooling of adjacent kernel outputs, and batch normalisation, to produce a value for each trained class, representing the belief that the class is present in the input slice. The learned parameters include the kernel functions and the connection weights of a final layer which combines kernel outputs into class predictions. This example summary only skims the surface of the various network architectures and layers available, and for an in-depth review see e.g. Khan et al. (2020). 


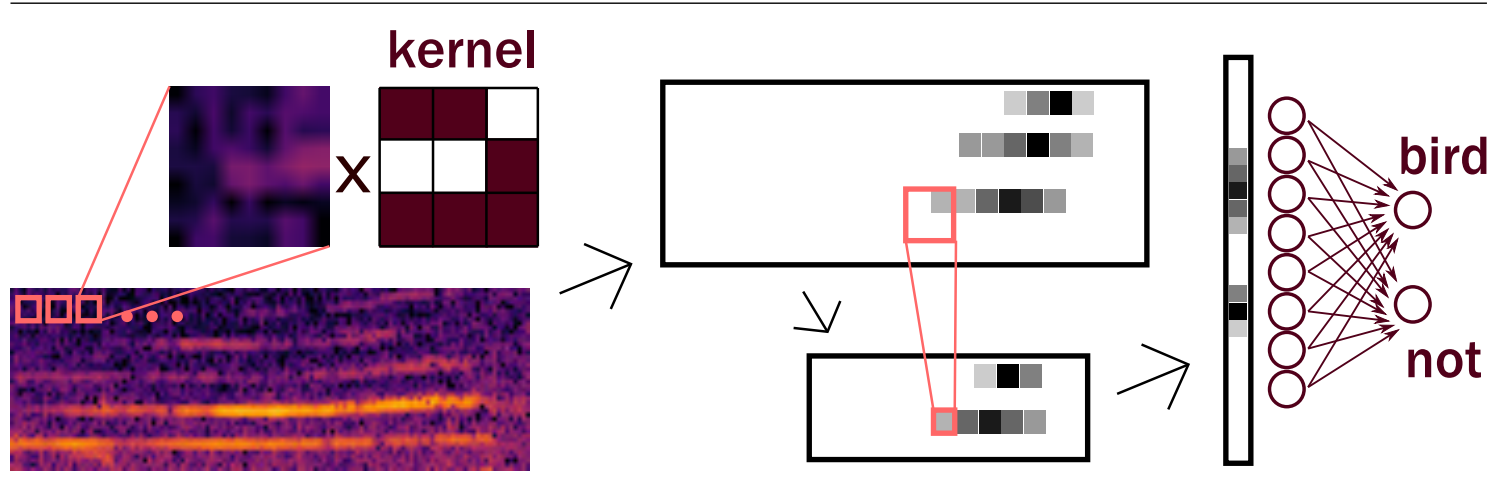

Figure 4: Scheme of a primitive CNN. A small kernel is convolved with a spectrogram (left), producing a 2D output (center), with darker pixels here indicating areas where the spectrogram matches the kernel's template better. To reduce its resolution, neighbouring pixels are pooled, e.g. by choosing their maximum value. The output is then treated as a vector and passed to a fully connected layer, which maps it to values for each class. Multiple kernels and layers are used in real CNNs, leading to millions of parameters.

The main focus of current developments is improving the detection accuracy in natural environments. While for clean, loud calls accurate species-level recognition has been possible for several years now, the performance dramatically drops when moving to noisy and sparse recordings (Goëau et al., 2016), and has remained a challenge since (Kahl et al., 2020). Some recently explored approaches to overcome this are: testing different network architectures and meta-structures (Ibrahim et al., 2021; Dufourq et al., 2021; Merchan et al., 2020), adding separate networks for noise learning and removal (Vickers et al., 2021), optimising spectrogram pre-processing for CNNs (Knight et al., 2019; Merchan et al., 2020), learning more robust features directly from the waveform (Sanchez et al., 2021). These studies have reported good performance on their respective target species.

However, achieving good results with CNNs so far has required thousands of labelled examples for each species; see e.g. Zhong et al. (2020); Dufourq et al. (2021). Collecting such datasets is difficult and expensive in bioacoustics. Possible ways to reduce this need include incorporating partially labelled data (Serizel et al., 2018; Zhong et al., 2020); transfer learning, i.e. starting with weights pre-trained with other data (Zhong et al., 2020; Kahl et al., 2021); augmenting the dataset with different perturbations of the annotated files (Nanni et al., 2019). Still, little is known about generalisability of such black-box models beyond the tested conditions, and what features are important for recognizing calls. This means that for studies in different environments, on different species, or at different levels of classification (e.g. distinguishing individuals) the training process currently must be repeated, requiring large labelling effort even with the data reduction methods. 
A second limitation of the CNN framework is that it detects by classifying. This is compatible with occupancy or presence-absence models, but any inference based on individual call timestamps will require additional post-processing, as in e.g. Dufourq et al. (2021). Procedures for this step have received less attention, and are commonly ad hoc, even in tasks that specifically evaluate timestamp accuracy (Serizel et al., 2018). Variations of neural networks that specifically focus on this aspect have been proposed (Rasmussen and Širović, 2021), and could be further explored in acoustics.

Despite the complexity of the development process, large data requirements, and concerns about generalisability, the research of neural networks in bioacoustics features good practices. Standardised tasks and public datasets, ablation studies investigating the importance of each step in the workflow, and open sharing of trained models such as the BirdNET (Kahl et al., 2021) allows easier comparisons and may lead to consensus methods and practices in bioacoustic analysis.

\subsection{Practical literature}

Many current studies that are using acoustics to answer biological or ecological questions are not using the complex neural network methods described above. In a typical workflow, target peaks are detected by simple thresholding of spectrogram or waveform intensity, such as:

$$
r(t)=\mathbb{1}\left(I_{f, t}^{2}>\mu_{I}+\lambda \sigma_{I}\right)
$$

where $\mathbb{1}$ is the indicator function, $\mu_{I}, \sigma_{I}$ are the mean and SD of the squared spectrogram $I_{f, t}^{2}$, and $\lambda$ a fixed threshold. This is followed by additional rules to combine the binary series $r(t)$ into presence segments of reasonable duration, which are then further checked by simple classifiers; some examples are Risch et al. (2019); Hendricks et al. (2021); Larsen et al. (2021). Variations of this framework differ in the pre-processing of spectrogram, denoising, selection of parameter $\lambda$, classifier features and complexity, and in the manner of converting the peaks into segments.

Template-based detection is also popular (Katz et al., 2016; Szymański et al., 2021; Buehler et al., 2021). A template spectrogram $\left(Z_{f, t}\right)_{f \leq L / 2, t<N}$ matched to the target is created, and cross-correlated with the analysed spectrogram $I_{f, t}$ :

$$
\rho(t)=\sum_{i=0}^{L / 2} \sum_{j=0}^{N-1} \frac{\left(Z_{i, j}-\mu_{Z}\right)\left(I_{i, j+t}-\mu_{I}\right)}{\sigma_{Z} \sigma_{I}}
$$


where $\mu, \sigma$ are the mean and SD of the relevant slices of each spectrogram. As with intensity, the score $\rho(t)$ is thresholded and peaks are merged into contiguous segments. A similar principle can be applied with binarised spectrograms (Katz et al., 2016). The template methods are more specific than generic intensity thresholding, but naturally require that the target sounds match the template well, and small variations in pitch or duration can drastically reduce the match score. (This is in fact a symptom of a more general problem - that pointwise differences between spectrograms do not represent the perceptual distance between their sounds (Turian and Henry, 2020).)

Performance of these methods, even combined with the classifiers, is not perfect (e.g. Marchal et al. (2021), Buehler et al. (2021)) and the outputs are still almost always verified manually. Nonetheless, their simplicity makes them attractive in practice: these methods can be trained with little data, and easy to implement or modify without extensive computational resources or skills.

However, comparisons between different methods in this type of studies are rare. As the methods here are often developed from the ground for a particular experiment, the procedures and parameters end up heavily tuned to a specific species, e.g. Nieukirk et al. (2020). Naturally, researchers prefer evaluation tasks, datasets, and metrics tailored to each application: general datasets such as the xeno-canto database, commonly used in machine learning, likely do not represent the target species or population with sufficient coverage (Kahl et al., 2021), and tasks from the public competitions may not match the practical goals, as for example the "any bird" detection task from DCASE that does not require any finer level of accuracy (Stowell et al., 2018). A variety of different metrics is also used, with ambiguous definitions (Knight et al., 2017). These factors make it very difficult to compare the methods based on published results: for example, see the variety of datasets and metrics reviewed in Priyadarshani et al. (2018b) or Kowarski and Moors-Murphy (2020).

There are several studies that specifically attempted to compare existing analysis methods by testing them in the same evaluation methodology (Knight et al., 2017; Marchal et al., 2021). Some performance differences were identified. But, since the compared tools are based on different detection frameworks with many unique processing steps and parameters, no insights into the causes of such differences could be made, and the results remain limited to the particular signals and noises tested. 


\section{The need for statistically principled processing tools}

Our goal in this thesis is to create sound processing tools that combine the best features from both groups of literature identified in the previous section. Namely, we seek methods for sound denoising, detection, classification, that:

- focus on solving ecological problems in practically realistic low-data settings, and

- are developed and evaluated in a principled way that allows to unambiguously identify improvements and limitations.

Furthermore, these methods must be compatible with the subsequent inference as reviewed in this chapter. To do this, we will develop our tools taking a statistically guided approach.

\subsection{Statistical signal theory}

Most existing processing methods stem from the theory of composite hypothesis testing, in which observations $\mathbf{X}=X(t), t=1, \ldots, T$ have different distributions under the noise-only and noisy-signal hypotheses (Levy, 2010):

$$
\begin{aligned}
& H_{0}: \mathbf{X} \sim f_{N}(\mathbf{x}), f_{N} \in \mathscr{F}_{N} \\
& H_{1}: \mathbf{X} \sim f_{S+N}(\mathbf{x}), f_{S+N} \in \mathscr{F}_{S+N}
\end{aligned}
$$

The families $\mathscr{F}_{N}, \mathscr{F}_{S+N}$ are not limited to a single distribution each. The presence of signal is determined by testing a chosen statistic $S(\mathbf{X})$ against an acceptance region $\mathscr{S}_{0}$. False positive and false negative error rates of this test are defined as, respectively, $P_{F P}\left(S, \mathscr{S}_{0}\right)=P\left(S \notin \mathscr{S}_{0} \mid f_{N}\right)$ and $P_{F N}\left(S, \mathscr{S}_{0}\right)=P\left(S \in \mathscr{S}_{0} \mid f_{S+N}\right)$. From these, criteria for an ideal test can be defined, such as uniformly most powerful testing of size $\alpha$ :

$$
\begin{aligned}
& \forall S^{\prime}, \mathscr{S}_{0}^{\prime}, f_{S+N}: P_{F N}\left(S, \mathscr{S}_{0}\right) \leq P_{F N}\left(S^{\prime}, \mathscr{S}_{0}^{\prime}\right) \\
& \quad \text { subject to } \max _{f_{N}} P_{F P}\left(S, \mathscr{S}_{0}\right) \leq \alpha, \max _{f_{N}} P_{F P}\left(S^{\prime}, \mathscr{S}_{0}^{\prime}\right) \leq \alpha
\end{aligned}
$$

For example, this criterion leads to thresholding $\sum_{i=1}^{T} X^{2}(t)$ for detecting increase in variance of a Gaussian (Lehmann and Romano, 2005). 
Relation of these principles specifically to acoustics has been discussed in the early literature (Altes, 1980; Middleton, 1987; Boashash and O'Shea, 1990). However, direct applications of such frameworks to sound are limited because the distributions involved are complex, dynamic, and often unknown. Furthermore, the hypothesis testing framework does not match many bioacoustic tasks, in particular those which require estimating precise positions of calls. As a result, modern analysis methods have become considerably more complicated, involving a number of ad hoc steps, and no longer based on an explicit model of signal or noise.

Meanwhile, considerable advances in the statistical signal processing are being made, such as algorithms for consistent detection of multiple transient signals (Truong et al., 2020) and adaptive time-frequency decompositions (Mallat, 2009). These advances could be used to build more effective, but still statisticallyprincipled sound analysis tools. Restoring the link to explicit models would make it easier to analyse and compare the resulting tools, clarify their constraints, generalisability and optimality conditions. Finally, this approach will likely limit us to simpler models, which matches the practical needs of small training data and low computational complexity.

\subsection{Objectives of this thesis}

The specific objectives of this work are:

1. Identify an evaluation framework, including a standard task and metric, that could be used to quantify differences between various sound processing methods with respect to ecological inference goals (Study I).

2. Design an improved method for detection and temporal localisation of sound events, within practical complexity and training data constraints (Studies II and III).

3. Create a method for estimating transient broadband noise, for use in robust sound event detection or denoising (Study IV).

These studies are presented as the next four chapters 2-5.

\section{AviaNZ software}

Central to all sound analyses presented in this thesis is the AviaNZ software, developed in our group (Marsland et al., 2019; Priyadarshani et al., 2020). Because 
human effort is still the main bottleneck for acoustic surveys - both in preparing annotated training data, and in reviewing automatic detections - creating an environment where these tasks can be done efficiently is crucial. This need has become more appreciated recently; Arce-Lopera et al. (2021) provide some examples of efficient user interface for these tasks. Therefore, a considerable part of work on this thesis was developing and maintaining the AviaNZ software, as well as providing support and workshops for using it.

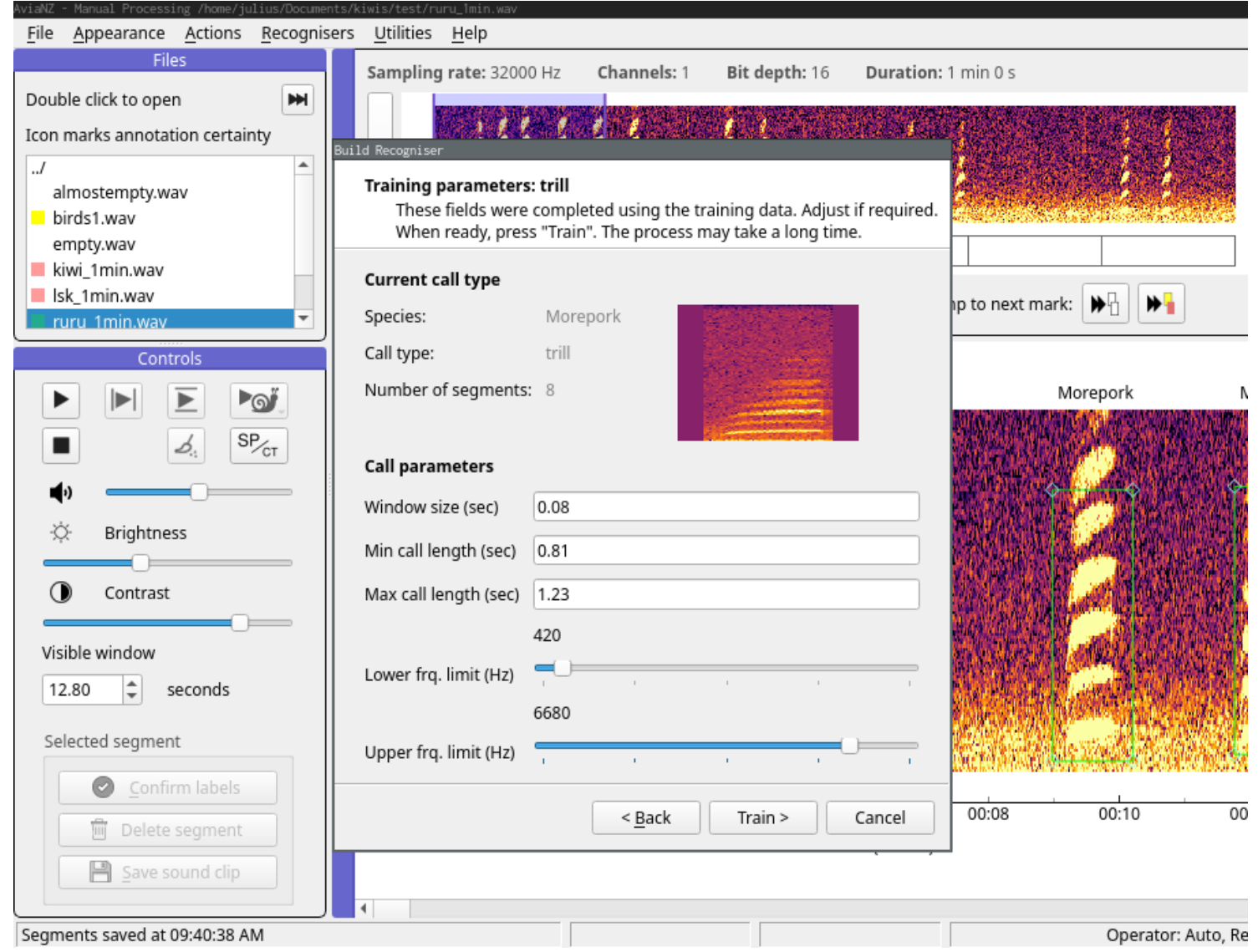

Figure 5: AviaNZ interface, showing the manually created annotations of calls (in the background) and a dialog for training an automated detector. Note that only interpretable parameters are required to be set by the user - remaining ones are trained automatically when "Train" is pressed.

AviaNZ provides a graphical interface for viewing, playing, analysing and annotating the sound files (Figure 5). Various detection methods are implemented, in a trainable form: users can tune the parameters to target any species, population or call type, using their own annotated data. It is free and open-source, available for common operating systems at www . avianz . net and github.com/smarsland/ AviaNZ. Its aim is to make the state-of-the-art tools accessible to practitioners, without requiring specialised knowledge, high-performance computing resources 
or expensive software.

The framework of this software also influenced our choice of analysis methods. For example, shifting the task of parameter tuning to the end user, who is not assumed to have intricate knowledge of the analysis mechanism, means that all such parameters must be either simple and interpretable (see Figure 5), or trainable without any user intervention. The latter case is surprisingly challenging, and requires optimisation methods that are largely independent of the nature of data at hand (Huang et al., 2020). Currently, key parameters in AviaNZ are determined by a combination of grid and iterative (stepwise) search (Priyadarshani et al., 2020). As before, these constraints favour small, interpretable models, so that the search space and optimal strategies could be determined in advance.

In terms of the included sound analysis tools, a particular feature distinguishing AviaNZ from similar software is its choice of wavelet packets as the main representation of sound. The wavelet packet is a basis comprised of compact functions that resemble short fragments of waves of various periods and centered at various times; projecting the sound waveform onto this basis decomposes it into a number of time-frequency components, and thus functions as an alternative to STFT (Mallat, 2009; Priyadarshani et al., 2016, 2020). While wavelet representations are used for sound detection throughout this work, the survey evaluation principles developed in Chapter 2 are not specific to this transform. Method developments in chapters 4 and 5 rely on wavelets more directly, and will present a more technical summary of their relevant properties.

\section{Author contributions}

The studies in chapters $2-5$ are joint works with I.C., N.P. and S.M. The contribution of J.J. to each of these manuscripts is in the capacity of the corresponding author, involving conception of ideas, manuscript drafting, implementing methods and proofs, contributing to data collection, designing and performing data analysis, and result interpretation. I.C. contributed to conception of ideas, result interpretation, and manuscript editing. N.P. contributed to conception of ideas, data collection, result interpretation, and manuscript editing. S.M. designed and conducted data collection, contributed to conception of ideas, designing data analysis, implementation of proofs, result interpretation, and manuscript editing. 


\section{Bibliography}

Altes, R. A. (1980) Detection, estimation, and classification with spectrograms. The Journal of the Acoustical Society of America, 67, 1232-1246.

Arce-Lopera, C., García-Muñoz, P., Restrepo-Quiceno, S., Gómez-Marín, D. and Londoño, G. A. (2021) Designing data visualization assistance for a bioacoustics labeling software. In HCI International 2021 - Posters (eds. C. Stephanidis, M. Antona and S. Ntoa), 475482. Cham: Springer International Publishing.

Baumgartner, M. F., Bonnell, J., Parijs, S. M. V., Corkeron, P. J., Hotchkin, C., Ball, K., Pelletier, L.-P., Partan, J., Peters, D., Kemp, J., Pietro, J., Newhall, K., Stokes, A., Cole, T. V. N., Quintana, E. and Kraus, S. D. (2019) Persistent near real-time passive acoustic monitoring for baleen whales from a moored buoy: System description and evaluation. Methods in Ecology and Evolution, 10, 1476-1489.

Boashash, B. and O'Shea, P. (1990) A methodology for detection and classification of some underwater acoustic signals using time-frequency analysis techniques. IEEE Transactions on Acoustics, Speech, and Signal Processing, 38, 1829-1841.

Borchers, D. L., Stevenson, B. C., Kidney, D., Thomas, L. and Marques, T. A. (2015) A unifying model for capture-recapture and distance sampling surveys of wildlife populations. Journal of the American Statistical Association, 110, 195-204.

Bradfer-Lawrence, T., Bunnefeld, N., Gardner, N., Willis, S. G. and Dent, D. H. (2020) Rapid assessment of avian species richness and abundance using acoustic indices. Ecological Indicators, 115, 106400.

Buckland, S. T., Rexstad, E. A., Marques, T. A. and Oedekoven, C. S. (2015) Modelling detection functions. In Distance Sampling: Methods and Applications, 53-103. Springer International Publishing.

Buehler, D. A., Fischer, R. A., Wilkerson, J. B., Worley, S., Smith, D. R., Hockman, E. and Prevost, S. (2021) Demonstration of autonomous aerial acoustic recording systems to inventory department of defense bird populations. Tech. rep., US Army Engineer Research and Development Center.

Charish, R., Berrow, S. and O'Brien, J. (2021) Acoustic monitoring of a bottlenose dolphin (Tursiops truncatus) population: Trends in presence and foraging beyond the limits of the lower river shannon SAC. Journal of Marine Science and Engineering, 9, 650.

Chelotti, J. O., Vanrell, S. R., Milone, D. H., Utsumi, S. A., Galli, J. R., Rufiner, H. L. and Giovanini, L. L. (2016) A real-time algorithm for acoustic monitoring of ingestive behavior of grazing cattle. Computers and Electronics in Agriculture, 127, 64-75.

Cheng, J., Sun, Y. and Ji, L. (2010) A call-independent and automatic acoustic system for the individual recognition of animals: A novel model using four passerines. Pattern Recognition, 43, 3846-3852. 
Clement, M. J., Rodhouse, T. J., Ormsbee, P. C., Szewczak, J. M. and Nichols, J. D. (2014) Accounting for false-positive acoustic detections of bats using occupancy models. Journal of Applied Ecology, 51, 1460-1467.

Collins, J. and Jones, G. (2009) Differences in bat activity in relation to bat detector height: Implications for bat surveys at proposed windfarm sites. Acta Chiropterologica, 11, 343350.

Dampage, U., Thajudeen, R., Jasenthuliyana, S. and Jayawardena, J. (2021) Automated virtual elephant fence based on detection, alarming, and coordinated redirection of wild elephants. Environmental Monitoring and Assessment, 193.

Dawson, D. and Bull, P. (1975) Counting birds in New Zealand forests. Notornis, 22, 101109.

Dawson, D. K. and Efford, M. G. (2009) Bird population density estimated from acoustic signals. Journal of Applied Ecology, 46, 1201-1209.

Dénes, F. V., Silveira, L. F. and Beissinger, S. R. (2015) Estimating abundance of unmarked animal populations: accounting for imperfect detection and other sources of zero inflation. Methods in Ecology and Evolution, 6, 543-556.

Dennis, J., Tran, H. D. and Li, H. (2011) Spectrogram image feature for sound event classification in mismatched conditions. IEEE Signal Processing Letters, 18, 130-133.

Digby, A. (2013) Whistling in the Dark: an Acoustic Study of Little Spotted Kiwi. Ph.D. thesis, Victoria University of Wellington.

Doser, J. W., Finley, A. O., Weed, A. S. and Zipkin, E. F. (2021) Integrating automated acoustic vocalization data and point count surveys for estimation of bird abundance. Methods in Ecology and Evolution, 12, 1040-1049.

Dufourq, E., Durbach, I., Hansford, J. P., Hoepfner, A., Ma, H., Bryant, J. V., Stender, C. S., Li, W., Liu, Z., Chen, Q., Zhou, Z. and Turvey, S. T. (2021) Automated detection of Hainan gibbon calls for passive acoustic monitoring. Remote Sensing in Ecology and Conservation.

Dunlop, R. A., Cato, D. H. and Noad, M. J. (2014) Evidence of a Lombard response in migrating humpback whales (Megaptera novaeangliae). The Journal of the Acoustical Society of America, 136, 430-437.

Eldridge, A., Guyot, P., Moscoso, P., Johnston, A., Eyre-Walker, Y. and Peck, M. (2018) Sounding out ecoacoustic metrics: Avian species richness is predicted by acoustic indices in temperate but not tropical habitats. Ecological Indicators, 95, 939-952.

Farina, A., Pieretti, N. and Piccioli, L. (2011) The soundscape methodology for long-term bird monitoring: A Mediterranean Europe case-study. Ecological Informatics, 6, 354-363.

Goëau, H., Glotin, H., Vellinga, W.-P., Planqué, R. and Joly, A. (2016) LifeCLEF bird identification task 2016: The arrival of deep learning. In CLEF (Working Notes) (eds. K. Balog, L. Cappellato, N. Ferro and C. Macdonald), vol. 1609 of CEUR Workshop Proceedings, 440-449. CEUR-WS.org. 
Haeb-Umbach, R., Watanabe, S., Nakatani, T., Bacchiani, M., Hoffmeister, B., Seltzer, M. L., Zen, H. and Souden, M. (2019) Speech processing for digital home assistants: Combining signal processing with deep-learning techniques. IEEE Signal Processing Magazine, 36, 111-124.

Hahn, A. H., Krysler, A. and Sturdy, C. B. (2013) Female song in black-capped chickadees (Poecile atricapillus): Acoustic song features that contain individual identity information and sex differences. Behavioural Processes, 98, 98-105.

Hendricks, B., Keen, E. M., Shine, C., Wray, J. L., Alidina, H. M. and Picard, C. R. (2021) Acoustic tracking of fin whales: Habitat use and movement patterns within a Canadian Pacific fjord system. The Journal of the Acoustical Society of America, 149, 4264-4280.

Huang, C., Li, Y. and Yao, X. (2020) A survey of automatic parameter tuning methods for metaheuristics. IEEE Transactions on Evolutionary Computation, 24, 201-216.

Ibrahim, A. K., Zhuang, H., Chérubin, L. M., Erdol, N., O'Corry-Crowe, G. and Ali, A. M. (2021) A multimodel deep learning algorithm to detect North Atlantic right whale upcalls. The Journal of the Acoustical Society of America, 150, 1264-1272.

Jeliazkov, A., Bas, Y., Kerbiriou, C., Julien, J.-F., Penone, C. and Viol, I. L. (2016) Large-scale semi-automated acoustic monitoring allows to detect temporal decline of bush-crickets. Global Ecology and Conservation, 6, 208-218.

Kahl, S., Clapp, M., Hopping, W., Goëau, H., Glotin, H., Planqué, R., Vellinga, W.-P. and Joly, A. (2020) Overview of BirdCLEF 2020: Bird sound recognition in complex acoustic environments. In CLEF 2020 - 11th International Conference of the Cross-Language Evaluation Forum for European Languages.

Kahl, S., Wood, C. M., Eibl, M. and Klinck, H. (2021) BirdNET: A deep learning solution for avian diversity monitoring. Ecological Informatics, 61, 101236.

Kalan, A. K., Mundry, R., Wagner, O. J., Heinicke, S., Boesch, C. and Kühl, H. S. (2015) Towards the automated detection and occupancy estimation of primates using passive acoustic monitoring. Ecological Indicators, 54, 217-226.

Kalmár, G., Wittemyer, G., Völgyesi, P., Rasmussen, H. B., Maróti, M. and Lédeczi, Á. (2019) Animal-borne anti-poaching system. In Proceedings of the 17th Annual International Conference on Mobile Systems, Applications, and Services. ACM.

Kasten, E. P., Gage, S. H., Fox, J. and Joo, W. (2012) The remote environmental assessment laboratory's acoustic library: An archive for studying soundscape ecology. Ecological Informatics, 12, 50-67.

Katz, J., Hafner, S. D. and Donovan, T. (2016) Tools for automated acoustic monitoring within the R package monitoR. Bioacoustics, 25, 197-210.

Khan, A., Sohail, A., Zahoora, U. and Qureshi, A. S. (2020) A survey of the recent architectures of deep convolutional neural networks. Artificial Intelligence Review, 53, 54555516. 
Knight, E., Hannah, K., Foley, G., Scott, C., Brigham, R. and Bayne, E. (2017) Recommendations for acoustic recognizer performance assessment with application to five common automated signal recognition programs. Avian Conservation and Ecology, 12.

Knight, E. C. and Bayne, E. M. (2018) Classification threshold and training data affect the quality and utility of focal species data processed with automated audio-recognition software. Bioacoustics, 28, 539-554.

Knight, E. C., Hernandez, S. P., Bayne, E. M., Bulitko, V. and Tucker, B. V. (2019) Preprocessing spectrogram parameters improve the accuracy of bioacoustic classification using convolutional neural networks. Bioacoustics, 29, 337-355.

Kobayashi, H. H., Kudo, H., Glotin, H., Roger, V., Poupard, M., Shimotoku, D., Fujiwara, A., Nakamura, K., Saito, K. and Sezaki, K. (2018) A real-time streaming and detection system for bio-acoustic ecological studies after the Fukushima accident. In Multimedia Tools and Applications for Environmental \& Biodiversity Informatics (eds. A. Joly, S. Vrochidis, K. Karatzas, A. Karppinen and P. Bonnet), 53-66. Cham: Springer International Publishing.

Kowarski, K. A. and Moors-Murphy, H. (2020) A review of big data analysis methods for baleen whale passive acoustic monitoring. Marine Mammal Science, 37, 652-673.

Larsen, A. S., Schmidt, J. H., Stapleton, H., Kristenson, H., Betchkal, D. and McKenna, M. F. (2021) Monitoring the phenology of the wood frog breeding season using bioacoustic methods. Ecological Indicators, 131, 108142.

Law, B. S., Brassil, T., Gonsalves, L., Roe, P., Truskinger, A. and McConville, A. (2018) Passive acoustics and sound recognition provide new insights on status and resilience of an iconic endangered marsupial (koala Phascolarctos cinereus) to timber harvesting. PLOS ONE, 13, e0205075.

Lehmann, E. L. and Romano, J. P. (2005) Uniformly most powerful tests. In Testing Statistical Hypotheses, 56-109. Springer New York.

Levy, B. C. (2010) Principles of Signal Detection and Parameter Estimation. Springer US.

Lin, T.-H. and Tsao, Y. (2019) Source separation in ecoacoustics: a roadmap towards versatile soundscape information retrieval. Remote Sensing in Ecology and Conservation, 6, $236-247$.

MacKenzie, D. I., Nichols, J. D., Lachman, G. B., Droege, S., Royle, J. A. and Langtimm, C. A. (2002) Estimating site occupancy rates when detection probabilities are less than one. Ecology, 83, 2248-2255.

Mallat, S. (2009) A Wavelet Tour of Signal Processing. Elsevier, Oxford.

Mallawaarachchi, A., Ong, S. H., Chitre, M. and Taylor, E. (2008) Spectrogram denoising and automated extraction of the fundamental frequency variation of dolphin whistles. The Journal of the Acoustical Society of America, 124, 1159-1170.

Mammides, C., Goodale, E., Dayananda, S. K., Kang, L. and Chen, J. (2017) Do acoustic 
indices correlate with bird diversity? Insights from two biodiverse regions in Yunnan province, south China. Ecological Indicators, 82, 470-477.

Marchal, J., Fabianek, F. and Aubry, Y. (2021) Software performance for the automated identification of bird vocalisations: the case of two closely related species. Bioacoustics, $1-17$.

Marsland, S., Priyadarshani, N., Juodakis, J. and Castro, I. (2019) AviaNZ: A future-proofed program for annotation and recognition of animal sounds in long-time field recordings. Methods in Ecology and Evolution, 10, 1189-1195.

Merchan, F., Guerra, A., Poveda, H., Guzmán, H. M. and Sanchez-Galan, J. E. (2020) Bioacoustic classification of Antillean manatee vocalization spectrograms using deep convolutional neural networks. Applied Sciences, 10, 3286.

Metcalf, O. C., Barlow, J., Devenish, C., Marsden, S., Berenguer, E. and Lees, A. C. (2020) Acoustic indices perform better when applied at ecologically meaningful time and frequency scales. Methods in Ecology and Evolution, 12, 421-431.

Metcalf, O. C., Ewen, J. G., McCready, M., Williams, E. M. and Rowcliffe, J. M. (2019) A novel method for using ecoacoustics to monitor post-translocation behaviour in an endangered passerine. Methods in Ecology and Evolution, 10, 626-636.

Middleton, D. (1987) Channel modeling and threshold signal processing in underwater acoustics: An analytical overview. IEEE Journal of Oceanic Engineering, 12, 4-28.

Mortimer, J. and Greene, T. (2017) Investigating bird call identification uncertainty using data from processed audio recordings. New Zealand Journal of Ecology, 41.

Nanni, L., Maguolo, G. and Paci, M. (2019) Data augmentation approaches for improving animal audio classification. arXiv preprint: 1912.07756.

Nieukirk, S. L., Mellinger, D. K., Dziak, R. P., Matsumoto, H. and Klinck, H. (2020) Multiyear occurrence of sei whale calls in North Atlantic polar waters. The Journal of the Acoustical Society of America, 147, 1842-1850.

Oedekoven, C. S., Marques, T. A., Harris, D., Thomas, L., Thode, A. M., Blackwell, S. B., Conrad, A. S. and Kim, K. H. (2021) A comparison of three methods for estimating call densities of migrating bowhead whales using passive acoustic monitoring. Environmental and Ecological Statistics.

Priyadarshani, N., Castro, I. and Marsland, S. (2018a) The impact of environmental factors in birdsong acquisition using automated recorders. Ecology and Evolution, 8, 5016-5033.

Priyadarshani, N., Marsland, S. and Castro, I. (2018b) Automated birdsong recognition in complex acoustic environments: a review. Journal of Avian Biology, 49, jav-01447.

Priyadarshani, N., Marsland, S., Castro, I. and Punchihewa, A. (2016) Birdsong denoising using wavelets. PLOS ONE, 11, e0146790.

Priyadarshani, N., Marsland, S., Juodakis, J., Castro, I. and Listanti, V. (2020) Wavelet filters for automated recognition of birdsong in long-time field recordings. Methods in Ecology and Evolution, 11, 403-417. 
Rasmussen, J. H. and Širović, A. (2021) Automatic detection and classification of baleen whale social calls using convolutional neural networks. The Journal of the Acoustical Society of America, 149, 3635-3644.

Rempel, R. S., Jackson, J. M., Wilgenburg, S. L. V. and Rodgers, J. A. (2019) A multiple detection state occupancy model using autonomous recordings facilitates correction of false positive and false negative observation errors. Avian Conservation and Ecology, 14.

Rhinehart, T. A., Chronister, L. M., Devlin, T. and Kitzes, J. (2020) Acoustic localization of terrestrial wildlife: Current practices and future opportunities. Ecology and Evolution, 10, 6794-6818.

Risch, D., Wilson, S. C., Hoogerwerf, M., van Geel, N. C. F., Edwards, E. W. J. and Brookes, K. L. (2019) Seasonal and diel acoustic presence of North Atlantic minke whales in the North Sea. Scientific Reports, 9.

Roca, I. T. and Opzeeland, I. V. (2019) Using acoustic metrics to characterize underwater acoustic biodiversity in the Southern Ocean. Remote Sensing in Ecology and Conservation, 6, 262-273.

Roe, P., Eichinski, P., Fuller, R. A., McDonald, P. G., Schwarzkopf, L., Towsey, M., Truskinger, A., Tucker, D. and Watson, D. M. (2021) The Australian Acoustic Observatory. Methods in Ecology and Evolution.

Ruppé, L., Clément, G., Herrel, A., Ballesta, L., Décamps, T., Kéver, L. and Parmentier, E. (2015) Environmental constraints drive the partitioning of the soundscape in fishes. Proceedings of the National Academy of Sciences, 112, 6092-6097.

Sanchez, F. J. B., Hossain, M. R., English, N. B. and Moore, S. T. (2021) Bioacoustic classification of avian calls from raw sound waveforms with an open-source deep learning architecture. Scientific Reports, 11.

Schmidt, A. K., Riede, K. and Römer, H. (2015) No phenotypic signature of acoustic competition in songs of a tropical cricket assemblage. Behavioral Ecology, 27, 211-218.

Serizel, R., Turpault, N., Eghbal-Zadeh, H. and Shah, A. P. (2018) Large-scale weakly labeled semi-supervised sound event detection in domestic environments. In Proceedings of the Detection and Classification of Acoustic Scenes and Events 2018 Workshop (DCASE2018), 19-23.

Shamon, H., Paraskevopoulou, Z., Kitzes, J., Card, E., Deichmann, J. L., Boyce, A. J. and McShea, W. J. (2021) Using ecoacoustics metrices to track grassland bird richness across landscape gradients. Ecological Indicators, 120, 106928.

Smith, D. G., Truskinger, A., Roe, P. and Watson, D. M. (2020) Do acoustically detectable species reflect overall diversity? A case study from Australia's arid zone. Remote Sensing in Ecology and Conservation, 6, 286-300.

Steel, Z. L., Campos, B., Frick, W. F., Burnett, R. and Safford, H. D. (2019) The effects of wildfire severity and pyrodiversity on bat occupancy and diversity in fire-suppressed forests. Scientific Reports, 9. 
Stevenson, B. C., Borchers, D. L., Altwegg, R., Swift, R. J., Gillespie, D. M. and Measey, G. J. (2015) A general framework for animal density estimation from acoustic detections across a fixed microphone array. Methods in Ecology and Evolution, 6, 38-48.

Stevenson, B. C., van Dam-Bates, P., Young, C. K. Y. and Measey, J. (2021) A spatial capture-recapture model to estimate call rate and population density from passive acoustic surveys. Methods in Ecology and Evolution, 12, 432-442.

Stowell, D., Wood, M. D., Pamuła, H., Stylianou, Y. and Glotin, H. (2018) Automatic acoustic detection of birds through deep learning: The first bird audio detection challenge. Methods in Ecology and Evolution, 10, 368-380.

Sugai, L. S. M., Silva, T. S. F., Ribeiro, J. W. and Llusia, D. (2018) Terrestrial passive acoustic monitoring: Review and perspectives. BioScience, 69, 15-25.

Szymański, P., Olszowiak, K., Wheeldon, A., Budka, M. and Osiejuk, T. S. (2021) Passive acoustic monitoring gives new insight into year-round duetting behaviour of a tropical songbird. Ecological Indicators, 122, 107271.

Towsey, M., Znidersic, E., Broken-Brow, J., Indraswari, K., Watson, D. M., Phillips, Y., Truskinger, A. and Roe, P. (2018) Long-duration, false-colour spectrograms for detecting species in large audio data-sets. Journal of Ecoacoustics, 2, 1-1.

Truong, C., Oudre, L. and Vayatis, N. (2020) Selective review of offline change point detection methods. Signal Processing, 167, 107299.

Turian, J. and Henry, M. (2020) I'm sorry for your loss: Spectrally-based audio distances are bad at pitch. In "I Can't Believe It's Not Better!" NeurIPS 2020 workshop.

Vickers, W., Milner, B., Risch, D. and Lee, R. (2021) Robust North Atlantic right whale detection using deep learning models for denoising. The Journal of the Acoustical Society of America, 149, 3797-3812.

Watson, M. J., Wilson, D. R. and Mennill, D. J. (2016) Anthropogenic light is associated with increased vocal activity by nocturnally migrating birds. The Condor, 118, 338-344.

Whittington, J., Shepherd, B., Forshner, A., St-Amand, J., Greenwood, J. L., Gillies, C. S., Johnston, B., Owchar, R., Petersen, D. and Rogala, J. K. (2019) Landbird trends in protected areas using time-to-event occupancy models. Ecosphere, 10.

Wrege, P. H., Rowland, E. D., Keen, S. and Shiu, Y. (2017) Acoustic monitoring for conservation in tropical forests: examples from forest elephants. Methods in Ecology and Evolution, 8, 1292-1301.

Wright, W. J., Irvine, K. M., Almberg, E. S. and Litt, A. R. (2019) Modelling misclassification in multi-species acoustic data when estimating occupancy and relative activity. Methods in Ecology and Evolution, 11, 71-81.

Xie, J., Indraswari, K., Schwarzkopf, L., Towsey, M., Zhang, J. and Roe, P. (2018) Acoustic classification of frog within-species and species-specific calls. Applied Acoustics, 131, 7986. 
Zeppelzauer, M., Hensman, S. and Stoeger, A. S. (2014) Towards an automated acoustic detection system for free-ranging elephants. Bioacoustics, 24, 13-29.

Zhong, M., LeBien, J., Campos-Cerqueira, M., Dodhia, R., Ferres, J. L., Velev, J. P. and Aide, T. M. (2020) Multispecies bioacoustic classification using transfer learning of deep convolutional neural networks with pseudo-labeling. Applied Acoustics, 166, 107375.

Znidersic, E., Towsey, M., Roy, W., Darling, S. E., Truskinger, A., Roe, P. and Watson, D. M. (2020) Using visualization and machine learning methods to monitor low detectability species-the least bittern as a case study. Ecological Informatics, 55, 101014. 


\title{
Precision as a metric for acoustic survey design using occupancy or spatial capture- recapture
}

\author{
Julius Juodakis ${ }^{1}$, Isabel Castro, Stephen Marsland
}

Published in Environmental and Ecological Statistics, 28, 587-608 (2021), by Springer Nature. Reproduced with permission from Springer Nature

\begin{abstract}
Passive acoustic surveys provide a convenient and cost-effective way to monitor animal populations, and methods for conducting and analysing such surveys are undergoing rapid development. However, no standard metric exists to evaluate the proposed changes. Furthermore, the metrics that are commonly used are specific to a single stage of the survey workflow, and may not reflect the overall effects of a design choice.

Here, we attempt to define the effectiveness of acoustic surveys conducted in two common frameworks of population inference - occupancy modelling and spatially explicit capture-recapture (SCR). Specifically, we investigate precision as a possible metric of survey performance, but we observe that it does not lead to generally optimal designs in occupancy modelling. In contrast, precision of the SCR density estimate can be optimised with fewer experiment-specific parameters. We illustrate these issues using simulations.

We further demonstrate how SCR precision can be used to evaluate design choices on a field survey of little spotted kiwi (Apteryx owenii). We compare call

\footnotetext{
${ }^{1}$ Corresponding author. Email: julius. juodakis@sms . vuw. ac.nz. J. Juodakis and S. Marsland at School of Mathematics and Statistics, Victoria University of Wellington, New Zealand; I. Castro at Wildlife and Ecology Group, Massey University, Palmerston North, New Zealand.
} 
recognition by software and human experts. The resulting tradeoff between missed calls and faster data throughput was accurately captured with the proposed metric, while common metrics failed to identify optimal improvements and could be inflated by deleting data.

Due to the flexibility of SCR framework, the approach presented here can be applied to a wide range of different survey designs. As the precision is directly related to the power of subsequent inference, this metric evaluates design choices at the application level and captures tradeoffs that are missed by stage-specific metrics, enabling reliable comparison of survey methods.

Keywords Spatial capture-recapture; Occupancy modelling; Passive acoustics; Optimal survey design

\section{Introduction}

Sound has been used to monitor vocalising animal populations for many years. Vocal cues are counted in field surveys, and the counts are used directly as an index of population size, or as input to more complex models for inferring species spatial distribution, temporal trends, community diversity, or other properties (reviewed in Gibb et al. (2018)). Over the past two decades, this use of acoustics has been changed drastically by the development of autonomous recording units, ARUs (Brandes, 2008; Blumstein et al., 2011; Shonfield and Bayne, 2017; Darras et al., 2019).

In their most basic usage, the recorders act as a replacement for field observers in order to collect survey data at a lower cost (Lemckert et al., 2005; Williams et al., 2018), but they have great potential to open up entirely new monitoring possibilities, such as ultrasound range analysis for bats (Sugai et al., 2018), localisation of calling animals (Collier et al., 2010), or rapid processing with automated sound detectors (Potamitis et al., 2014). Even for simple surveys, the methodology of using ARUs is actively developed: studies have investigated the optimal number and placement of recorders (e.g., Pérez-Granados et al. (2018)), compared hardware (Rempel et al., 2013; Pérez-Granados et al., 2019), and optimised recording time and duration (Cook and Hartley, 2018; Hagens et al., 2018; Pérez-Granados et al., 2018).

The large amounts of data generated by ARUs has also sparked interest in algorithms to perform call denoising, detection and recognition (Acevedo et al., 2009; Priyadarshani et al., 2018; Stowell et al., 2018). However, no unified metric to 
evaluate these various developments exists. For example, denoising success is usually measured by signal-to-noise ratio (Priyadarshani et al., 2016) or by successful species classification after denoising (Connor et al., 2012). In turn, the species classification methods are evaluated by measuring their agreement with manual annotations, i.e., area under the receiver operating characteristic curve, accuracy, sensitivity, or specificity (see further below or Knight et al. (2017) for definitions). These measures can vary hugely between datasets: methods with claimed accuracy of $>90 \%$ can turn out to be only $40 \%$ accurate on a different dataset (Priyadarshani et al., 2018). Further complications arise because the target is not inherently binary - agreement can be measured per time unit, at the syllable level, call level, or file level, with different results (cf. Acevedo et al. (2009); Swiston and Mennill (2009); Towsey et al. (2012); Potamitis et al. (2014)). In fact, a perfect match with human annotations may not be achievable at all, given the variability in expert annotations (Mortimer and Greene, 2017). This lack of standardisation hampers any attempt to compare or improve the methods.

Another issue that complicates survey method comparison is that improvement in one particular stage of the process does not necessary correspond to more accurate or reliable monitoring overall. Compared to field observations, sound recorders have lower detection radius (Yip et al., 2017) and do not capture visual cues, but having recorded data provides many other benefits (Darras et al., 2019). Automated methods for annotation unavoidably miss some calls and likely introduce false positives, in return for faster processing, while additional data sources, such as radio tracking, may come at an extra cost and require sacrifices in sample size. These tradeoffs are not captured by stage-specific metrics. As summarised in Blumstein et al. (2011), "there is a growing need for a common framework in which to develop, run and fully evaluate new bioacoustic recognition systems. Such a framework would include standard performance metrics".

For single-species, single-population surveys, which estimate one quantity of ecological interest, a natural choice of metric is the final precision (i.e., the variance) of this estimate. This metric corresponds directly with the power of subsequent inference, such as detecting abundance changes or identifying predictors of species distribution (Stevenson et al., 2015; Metcalf et al., 2019), and thus would allow evaluating changes to any part of the survey protocol at the final level of ecological application. We focus on two popular statistical survey frameworks: occupancy models (which aim to estimate the fraction of area occupied by the population) and acoustic spatial capture-recapture (SCR), which estimates the density 
of animals in the survey area; these approaches are summarised in the next section. We investigate how the variance of such estimates can be used to evaluate changes in survey design.

Specifically, we describe the concept of optimal design in these two frameworks, based on the precision of the final estimate. In each case, we investigate how the optimum depends on unobserved parameters, using simulations and approximations recently developed by Efford and Boulanger (2019). In the second part of this paper, we focus on SCR. We use field data from a bird survey to demonstrate how the precision of density estimate can be used to evaluate design tradeoffs. We apply SCR to compare automatic call detection software with human listeners, and also show that other commonly-used metrics of classification accuracy can favour detrimental design choices. Finally, we discuss the remaining challenges that need to be overcome to enable standardised survey evaluation.

\section{Survey models and optimal design}

\subsection{Occupancy modelling}

Occupancy models (MacKenzie et al., 2002) seek to determine what fraction $\Psi$ of the survey area is occupied by the species of interest, based on $S$ monitoring devices (sites), such as acoustic recorders, placed in the area. To allow imperfect detection, a probability $p=P$ (species detected in site $s \mid$ species present in site $s$ ) is introduced; this can be interpreted as the sensitivity of the monitoring. To estimate both $p$ and $\Psi$, the survey is repeated $K$ times. The result is a binary detection matrix $\boldsymbol{\Omega}=\left\{\omega_{s k}\right\}$, indicating whether animals were detected at site $s$ on occasion $k$. It is summarised by two statistics: $s_{k}$, the number of sites where the species was detected on occasion $k$, and $\bar{s}$, the number of sites where the species was detected on at least one occasion (i.e., was present, assuming no false positives). The occupancy model then corresponds to this likelihood:

$$
L\left(\Psi, p \mid \bar{s}, s_{1}, \cdots, s_{k}\right)=\Psi^{\bar{s}} \prod_{k=1}^{K} p^{s_{k}}(1-p)^{\bar{s}-s_{k}} \times\left(\Psi(1-p)^{K}+1-\Psi\right)^{S-\bar{s}} .
$$

Various extensions to this model have been proposed, allowing, for example, different numbers of surveys at each site, and site-specific detection covariates (MacKenzie et al., 2002; Mackenzie and Royle, 2005; Clement, 2016). One such extension that will be useful here is to separate the detection probability into a 
detectability component $p_{d}$ and an availability component $p_{a}$, so that $p=p_{d} p_{a}$ (Riddle et al., 2010). Availability summarises behavioural factors that cannot be controlled by the researcher, such as the call rate of the target species, while detectability is the probability to capture emitted cues: for example, an ideal listener may be described with $p_{d}=1$ and still miss animals that did not vocalise during the survey. It is assumed here that both are constant over the survey, although spatial and temporal variations are likely in practice.

The ultimate aim of this framework is to enable inference about the occupancy parameter $\Psi$, hence a natural metric for evaluating survey efficacy is the sampling variance of this parameter, $\operatorname{Var}(\hat{\Psi})$ (throughout, we use $\hat{\cdot}$ to denote estimates). The researcher can then choose the experimental design that minimises this variance, and thus maximises the power for subsequent inference, while keeping the estimate unbiased. This approach is well-known in experimental design (Atkinson and Donev, 1992), and in occupancy modelling in particular (Mackenzie and Royle, 2005; Clement, 2016; Gálvez et al., 2016).

Formally, let $\boldsymbol{m} \in M$ be a vector containing all study parameters that are in control of the researcher. This may include allocation of sampling effort, i.e., $S$ and $K$, but also less obvious design choices, such as different monitoring device suppliers or observation protocols (which may result in different $p_{d}$ ). The researcher's goal is to determine the optimal study design, i.e.: $\operatorname{argmin}_{m \in M} \operatorname{Var}(\hat{\Psi})$.

This problem is made non-trivial by practical considerations, which constrain the space $M$. For example, often the total observer effort $S K$ is assumed to be fixed (Mackenzie and Royle, 2005), or budgetary constraints may force a choice between few good sensors (high $p_{d}$, low $S$ ) or many, but worse, sensors (low $p_{d}$, high $S$ ).

As the true values of the parameters $\Psi$ and $p_{a}$ are not controlled or known in advance, ideally the optimal design should be insensitive to them, i.e.:

$$
\underset{m \in M}{\operatorname{argmin}} \operatorname{Var}\left(\hat{\Psi} \mid \Psi=\Psi_{1}\right)=\underset{m \in M}{\operatorname{argmin}} \operatorname{Var}\left(\hat{\Psi} \mid \Psi=\Psi_{2}\right) \forall \Psi_{1}, \Psi_{2}
$$

which is true if and only if:

$$
\operatorname{Var}\left(\hat{\Psi} \mid \Psi=\Psi_{2}\right)=g\left(\operatorname{Var}\left(\hat{\Psi} \mid \Psi=\Psi_{1}\right)\right)
$$

for some strictly monotonic $g(x)>0$.

However, the occupancy model does not allow these parameters to be isolated as monotonic functions. Namely, by substituting $p=p_{a} p_{d}$ into the asymptotic variance 
formula from Mackenzie and Royle (2005), we obtain:

$$
\operatorname{Var}(\hat{\Psi})=\frac{\Psi K}{S K}\left(1-\Psi+\frac{\left(1-p_{a} p_{d}\right)^{K}}{1-\left(1-p_{a} p_{d}\right)^{K}-K p_{a} p_{d}\left(1-p_{a} p_{d}\right)^{K-1}}\right)
$$

Now, if we seek optimal $S, K$ given fixed $S K$, we have:

$$
\underset{m \in M}{\operatorname{argmin}} \operatorname{Var}(\hat{\Psi})=\underset{K}{\operatorname{argmin}} \operatorname{Var}(\hat{\Psi})=\underset{K}{\operatorname{argmin}}\left(K-K \Psi+K f\left(p_{a}, p_{d}, K\right)\right)
$$

where $f$ is a function that depends on its arguments in a complex way and is nonnegligible, unless $p_{a} p_{d} \rightarrow 1$. (Note that even then $\operatorname{Var}(\hat{\Psi})>0$ as it captures the variance over possible realisations of the occupancy.) Thus, the optimal choice of $K$ depends on the true values of $\Psi$ and $p_{a}$.

Similar results hold for other constraints. For example, a tradeoff between improved detection and more sampling effort can be represented by $M=\left\{\left(S, K, p_{d}\right) \mid S K p_{d}=\right.$ const $\}$. Then the minimisation target will take the form $-K p_{d} \Psi+K p_{d} f\left(p_{a}, p_{d}, K\right)$, again meaning that there is no general solution without prior knowledge of values of $p_{a}$ and $\Psi$.

In practice, estimating $\operatorname{Var}(\hat{\Psi})$ is further complicated by several issues. Formula (1) may underestimate the true variance due to differences between full and composite likelihood (Varin et al., 2011), as discussed in detail below. It could be replaced with empirical variance of parametric bootstrap replicates, but then boundary issues may still arise at low sample sizes or probabilities close to 0 or 1 . In the remainder of this paper, we assume that the asymptotic variance is accurate, to show that the results are inherent to the model and not a consequence of the estimation method.

\subsection{Spatial capture-recapture}

Spatially explicit capture-recapture (SCR, or SECR) is an alternative framework for data analysis of detections (Efford, 2004; Efford et al., 2009; Dawson and Efford, 2009; Borchers et al., 2015). The classic SCR model consists of three components. Density $D$ is the main inference target, and defines the density of animals in the survey area; it is taken to be constant over the space and time, or have some parameterised functional form. Animal home range centres $X=\left[\boldsymbol{x}_{1}, \cdots, \boldsymbol{x}_{N}\right]$ are random locations, generated based on $D$. These locations are unobserved. The observed data is a capture history $\boldsymbol{\Omega}=\left\{\omega_{s k n}\right\}$ where each $\omega_{s k n}=1$ if animal $n$ was detected at sensor $s$ on occasion $k$. It is assumed that the probability to observe an animal 
depends only on the distance $d_{s}(\boldsymbol{x})$ between its home range centre $\boldsymbol{x}$ and the detector $s$ - reasoning that the animal is less likely to be in proximity of detectors that are far from its home centre. An example of such detectors are hair snares: animals are detected if they wander into the trap, and multiple captures of the same individual can be identified based on DNA comparison (Boulanger et al., 2018).

Specifically, the locations $X$ are assumed to be realisations of a homogeneous Poisson process with intensity $D$, and the detection probability is chosen to be a function $p\left(d_{s}(\boldsymbol{x}) \mid \boldsymbol{\theta}\right)$ with some parameters $\boldsymbol{\theta}$. The resulting likelihood is of the form:

$$
L(D, \boldsymbol{\theta} \mid \boldsymbol{\Omega})=P(N \mid D, \boldsymbol{\theta}) \times \int_{\mathbb{R}^{2 N}} f_{X}(\boldsymbol{X} \mid D) P(\boldsymbol{\Omega} \mid \boldsymbol{X}, \boldsymbol{\theta}) d \boldsymbol{X},
$$

where $N$ is the number of animals observed, and $f_{X}$ the Poisson process pdf. The parameters $D$ and $\boldsymbol{\theta}$ are estimated by maximising (an approximation of) this likelihood. To make this feasible, the $2 N$-dimensional integral is replaced with a product of $N$ integrals of 2 dimensions, by assuming independence between $x$. This assumption is likely violated in practice, as territorial interactions between animals occur, but the resulting composite likelihood then still produces consistent estimates under some standard regularity conditions (Varin et al., 2011). However, it is less efficient, and the standard Hessian method in that case underestimates the sampling variance, requiring either adjusted variance estimators or bootstrap. More details on composite likelihood and the resulting variance estimation can be found in (Varin et al., 2011; Bai et al., 2012; Zhao et al., 2019). The integration is also approximated by summing over an 'integration mask', a grid of likely $\boldsymbol{x}$ values, but this can be chosen to be sufficiently large and dense to avoid approximation issues (Stevenson et al., 2015).

The basic SCR form is very flexible and can be extended to analyse diverse experimental designs and incorporate non-homogeneous density, various additional information collected by the sensors, and environment and behaviour covariates (Dawson and Efford, 2009; Reich and Gardner, 2014; Stevenson et al., 2015; Borchers et al., 2015; Boulanger et al., 2018).

For analysing acoustic surveys, the above model is not immediately applicable. The basic unit of acoustic SCR is a call: i.e., $\left\{\omega_{s k n}\right\}=1$ if call $n$ is detected on recorder $s$ on occasion $k$. Thus, two recordings of the same call comprise a 'capture' and 'recapture'. Such matches across recorders are usually identified based on temporal overlap. From this data, the density of calls $D$ and detection parameters are estimated by numeric likelihood maximisation, as before (see also Supplementary Figure S5 for an example of SCR input). 
Turning a call density into the underlying animal density $D_{a}$ requires either the ability to identify individuals in an unsupervised way, or prior knowledge of their call rate $\mu$, i.e., mean number of calls per individual per hour. The first is generally extremely difficult, and the second may well be hard to identify (see e.g., Digby (2013) for the little spotted kiwi, the species we focus on in the experimental section). However, if the true call rate $\mu$ was known, the animal density would be estimated as $\hat{D}_{a}=\hat{D} / \mu \tau$, where $\tau$ is the survey duration (Stevenson et al., 2015). Therefore, call density trends are used as proxy for population trends, assuming that the underlying call rate remains unchanged. We will review alternative solutions to this problem in the Discussion.

In designing SCR studies, precision of estimates has been used occasionally (e.g., Sun et al. (2014)), but without investigating the theoretical or empirical grounds for it, likely because no closed form expression for the variance of the estimates was available. For standard SCR, Efford and Boulanger (2019) recently derived an approximation to the sampling variance of $\hat{D}$, assuming Poisson-distributed number of unique captures $N$, and number of recapture events $R$, as:

$$
\begin{aligned}
\operatorname{Var}(\hat{D}) & \approx D^{2} / \min \{E(N), E(R)\}, \text { where } \\
E(N) & =\int_{\mathbb{R}^{2}}\left(1-\prod_{s=1}^{S} \prod_{k=1}^{K} p\left(d_{s}(\boldsymbol{x}) \mid \boldsymbol{\theta}\right)\right) D(\boldsymbol{x}) d \boldsymbol{x} \\
E(R) & =\int_{\mathbb{R}^{2}} \sum_{s=1}^{S} \sum_{k=1}^{K} p\left(d_{s}(\boldsymbol{x}) \mid \boldsymbol{\theta}\right) D(\boldsymbol{x}) d \boldsymbol{x}-E(N) .
\end{aligned}
$$

As previously, here $S$ and $K$ are the number of sensors and survey occasions, respectively, and $p$ is the detection function. Note that the formulation also allows inhomogeneous density $D(\boldsymbol{x})$.

To apply the above equations to acoustics, we assume that the density of calls is homogeneous and equal to $\mu D_{a} \tau$. We also drop the $K$ variable, as acoustic surveys are usually treated as single-occasion data, to obtain:

$$
\begin{aligned}
& E(N)=\int_{\mathbb{R}^{2}}\left(1-\prod_{s=1}^{S} p\left(d_{s}(\boldsymbol{x}) \mid \boldsymbol{\theta}\right)\right) \mu D_{a} \tau d \boldsymbol{x} \\
& E(R)=\int_{\mathbb{R}^{2}} \sum_{s=1}^{S} p\left(d_{s}(\boldsymbol{x}) \mid \boldsymbol{\theta}\right) \mu D_{a} \tau d \boldsymbol{x}-E(N) .
\end{aligned}
$$

Of the parameters discussed in this section, only $S, \tau$ and $\boldsymbol{\theta}$ may be controlled by the researcher. As before, practical constraints, such as total budget, limit their 
possible values to some space $M$. Thus, the task of optimising the study design $\boldsymbol{m}=(S, \tau, \boldsymbol{\theta})$ for acoustic SCR can be written as:

$$
\underset{\boldsymbol{m} \in M}{\operatorname{argmin}} \operatorname{Var}(\hat{D})=\underset{\boldsymbol{m} \in M}{\operatorname{argmin}} \frac{\left(\mu D_{a}\right)^{2}}{\mu D_{a} f(S, \tau, \boldsymbol{\theta})}=\underset{\boldsymbol{m} \in M}{\operatorname{argmin}} 1 / f(S, \tau, \boldsymbol{\theta}),
$$

and so the optimal design is invariant to the true density $D$ or call rate $\mu$ (or any other parameters that have a constant and uniform effect on the call density). Thus, in this respect, the design for acoustic surveys in SCR framework can be optimised without prior knowledge of behavioural parameters. As this result relies on several approximations, it will be verified by simulations next.

\section{Simulation experiments}

We use numeric examples to illustrate the different effects of unobserved parameters in both occupancy studies and SCR models. For the latter, the variance of the density estimator, discussed in the previous section, cannot be calculated analytically. We will therefore estimate it by the standard error of $\hat{D}$ calculated from simulated (this section) or real (further below) datasets.

\subsection{Setup}

To illustrate that the optimal design for occupancy studies depends on the values of population-specific parameters, we calculated the standard error of $\hat{\Psi}$ over several values of $p_{a}, p_{d}, \Psi$ and $K$. (Recall that $p_{d}$ captures the effect of design choices, such as a threshold for call detection, while $p_{a}$ cannot be manipulated by the observer.) We assumed fixed sampling effort of 120 sites, i.e., $S=120 / K$, and then calculated the asymptotic variance by substituting the true parameter values into Eq. (1).

As our results concerning SCR rely on the approximate variance formula, as well as independent call locations, we verified that they are valid for acoustic surveys using simulation. We used the ascr package to generate binary detection histories, as outlined in Stevenson et al. (2015). In summary, the user defines a survey by providing recorder positions and the detection function, as well as the true call density $D$. Call locations are then generated based on this density: in the independent case, locations are drawn from a homogeneous spatial Poisson process with intensity $D$. Alternatively, animals can be modelled to call repeatedly from the same position, with a user-set call rate $\mu$; then locations are drawn from a Poisson process with intensity $D / \mu$, and each location duplicated $\mu$ times. To create 'observed' 
histories at each detector, these locations are then filtered based on the distance to each detector and the provided detection parameters.

We used a half-normal detection function $p(d \mid \sigma)=g_{0} \exp \left(-d^{2} /\left(2 \sigma^{2}\right)\right)$, and a $3 \times 3,4 \times 4$ or $5 \times 5$ grid of detectors $(S)$ spaced at $200 \mathrm{~m}$. The integration mask was set to a $200 \mathrm{~m}$ buffer around the detectors. The design parameters corresponding to such a survey are $\boldsymbol{m}=\left(g_{0}, \sigma, S\right)$; note that $\sigma$ is aparameter controlling the detection function, but its changes can equivalently be interpreted as varying the spacing between detectors. We tested several combinations of values for these parameters, excluding cases where all three are at their best or worst values (to simulate realistic constraints on $M$, such as a tradeoff between more or better recorders). We did not vary the survey duration $\tau$ in the simulation - as the capture histories do not include actual call times, a longer survey at lower call rate would be indistinguishable from a shorter survey with higher call rate.

We initially modelled the call locations as independent. Simulations were conducted with different values of $D$ between 100 and 500. For each tested combination of design parameters and $D$ values, 100 survey datasets were generated, an SCR model fitted to each dataset producing estimates $\hat{D}^{* b}$ for $b=1, \ldots, 100$, and the observed standard deviation of $\hat{D}^{* b}$ used as an estimate of the corresponding SE. Relative bias was calculated as $\frac{1}{100} \sum_{b} \frac{\hat{D}^{* b}-D}{D}$.

To evaluate sensitivity to the independence assumption, we conducted a similar set of simulations with dependent locations. Several values of $\mu$ were tested, with a fixed call density $D=300,100$ replicates simulated, and each detection history analysed similarly to the independent case.

An example of a simulated detection history and other inputs to SCR is provided in Supplementary Figure S5.

\subsection{The effect of population parameters on experimental design}

It is generally known that optimal design for occupancy studies depends on the true occupancy (Mackenzie and Royle, 2005). We replicated this result (Figure 1). Separating the availability and detectability components also revealed that the optimal design depends on the availability component $p_{a}$. For example, designs with lower replication effort $(K=4)$ were optimal when $p_{a}=0.8, \Psi=0.5$, but they were the worst of the six tested designs when $p_{a}=0.4, \Psi=0.7$. Design ranking was not consistent across the other conditions either, and relatively small differences in parameter values were sufficient to cause drastic changes in design ranking. 


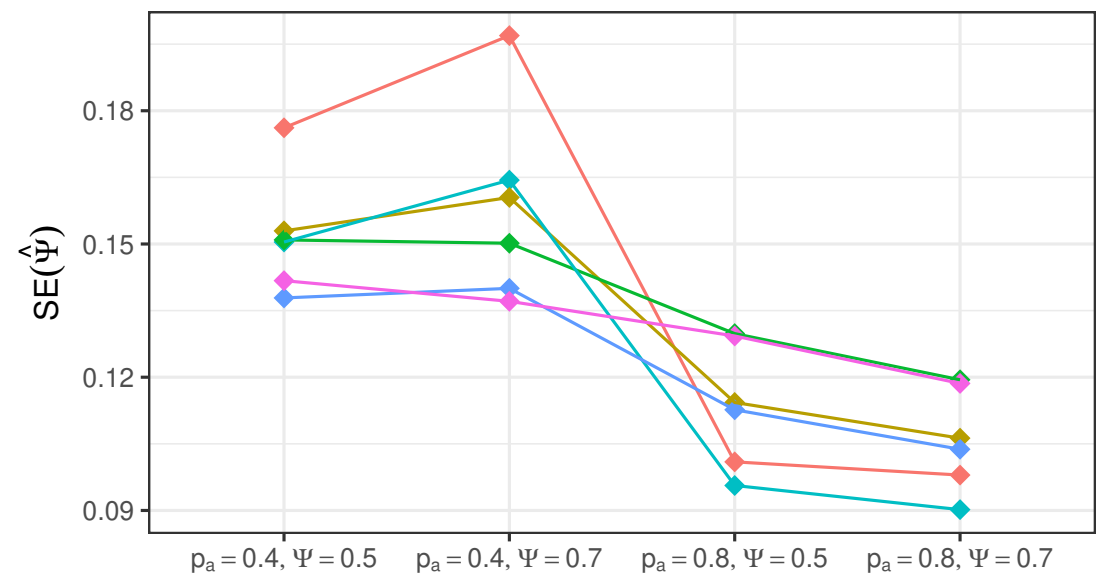

Design

$\neg p_{d}=0.6, K=4$

$\neg p_{d}=0.6, K=6$

$\neg p_{d}=0.6, K=8$

$\neg \mathrm{p}_{\mathrm{d}}=0.7, \mathrm{~K}=4$

$\neg p_{d}=0.7, K=6$

$\rightarrow p_{d}=0.7, K=8$

Figure 1: Standard error of the occupancy estimate $\hat{\Psi}$ for different combinations of unobserved parameters (availability $p_{a}$, true occupancy $\Psi$ ) and design choices (detectability $p_{d}$, survey occasions $K$ ). Note that the SEs are calculated analytically and thus no error bars are shown.

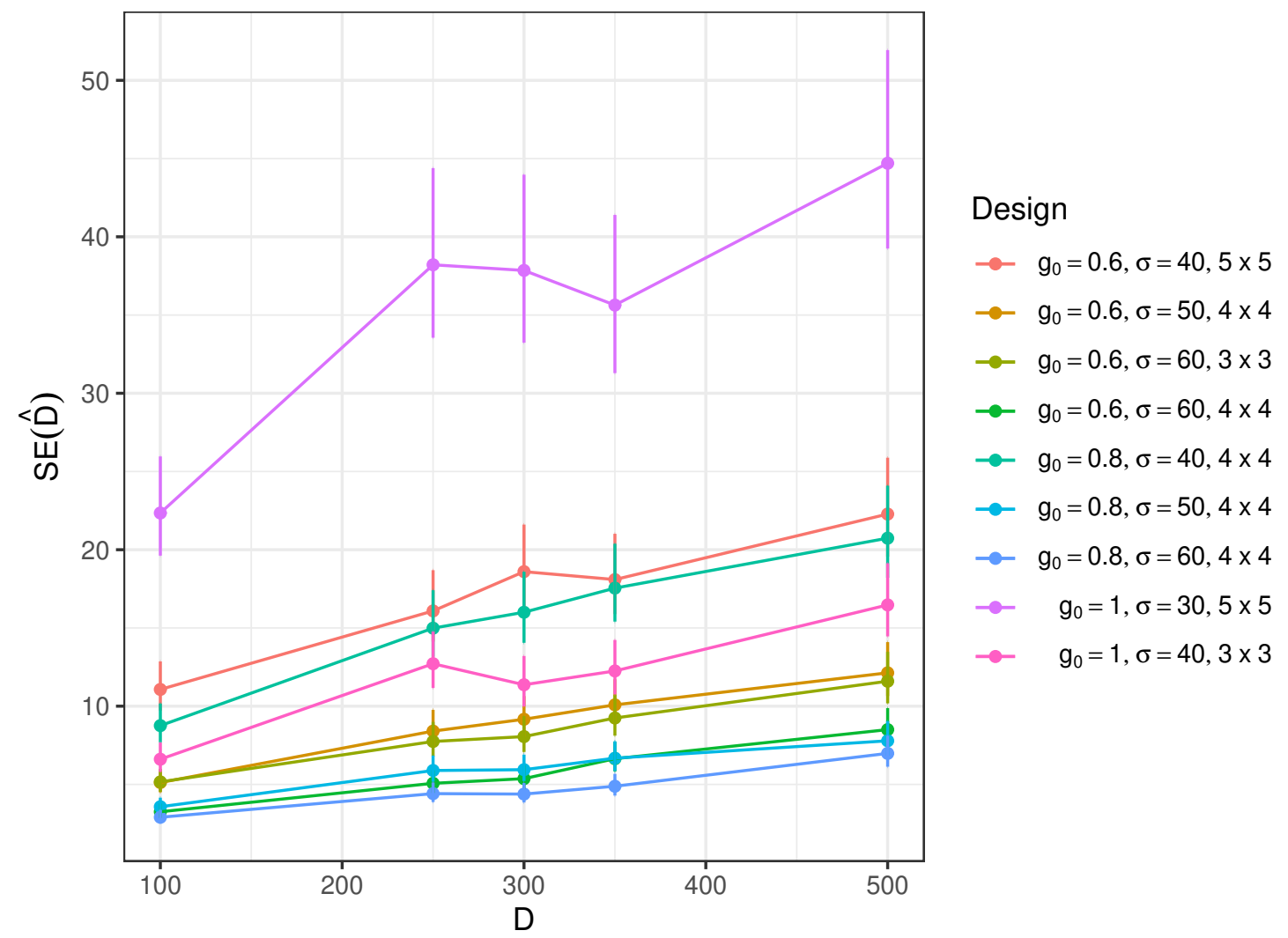

Figure 2: Standard error of SCR density estimate $\hat{D}$ for different values of true density $D$ and various design choices. Design choices are represented by different numbers of sensors and different parameters of the detection function. For each setting, capture histories were simulated, assuming independent call locations, and density estimated with SCR. Dots are the standard errors based on 100 replicates, and vertical bars indicate $95 \%$ confidence intervals based on $\chi^{2}$ distribution of the SE. Density units are calls per hectare per survey. 
In contrast, designs identified as optimal in the SCR framework are expected to remain optimal under a broad range of density values. Figure 2 shows the estimated precision obtained under 9 different study designs. The design with $g_{0}=0.8, \sigma=60$, and a $4 \times 4$ detector grid was optimal under all tested values of $D$; in general, the ranking of designs remained stable across the entire range of $D$ values tested. The design with $g_{0}=1, \sigma=30$ and a $5 \times 5$ grid was the worst in all conditions, and produced biased estimates (up to $15 \%$ lower; Supplementary Figure S10). All other designs estimated densities within $3 \%$ of the true value.

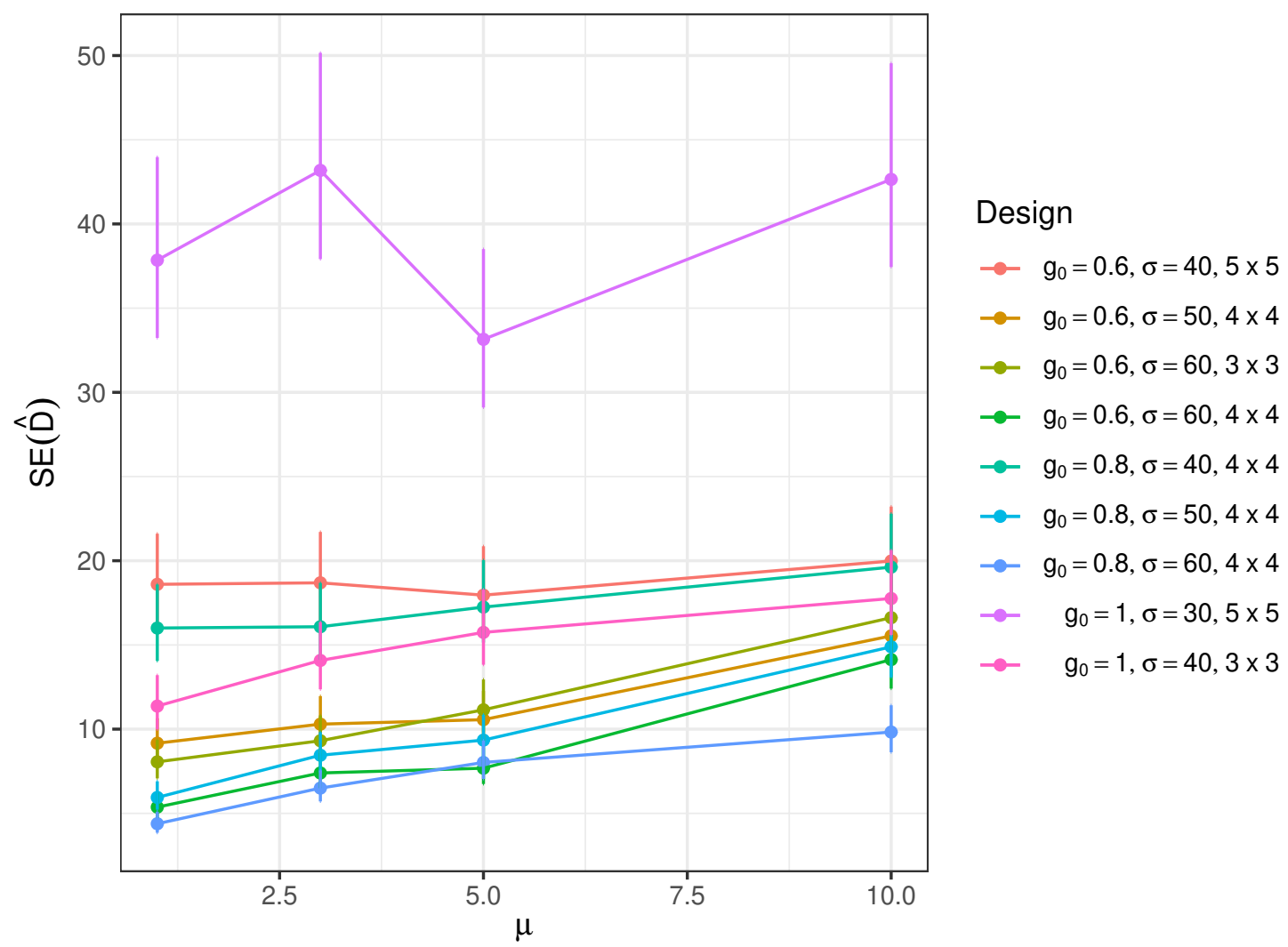

Figure 3: Standard error of SCR density estimate $\hat{D}$ for different values of true call rate $\mu$ and various design choices. Design choices are represented by different numbers of sensors and different parameters of the detection function. Capture histories were simulated for each setting, with $D=300$ and calls repeated $\mu$ times in each position. Dots are the standard errors based on 100 replicates, and vertical bars indicate $95 \%$ confidence intervals based on $\chi^{2}$ distribution of the SE. Density units are calls per hectare per survey.

Similar results were observed when dependency in call locations was introduced: relative efficiency of study designs for SCR remained stable across various values of call rate $\mu$ (Figure 3 ). Some minor deviations were observed, likely attributable to sampling variation in the standard error itself. Bias was also similar to the independent case, at most 3.4\%, apart from the pathological design, which 
had 5-10\% bias (Supplementary Figure S10).

Given the results in this section we chose to examine SCR precision as a metric further in the analysis of a real-world acoustic bird survey.

\section{Kiwi survey methods}

\subsection{Survey data and annotation}

To show how SCR can be used to evaluate design tradeoffs in a practical setting, we conducted an acoustic survey of little spotted kiwi (Apteryx owenii) in Zealandia, a 225 hectare wildlife sanctuary in Wellington, New Zealand. The likely population size is around 200 kiwi, with 40 kiwi translocated to this site in 2001, and approximately 100 estimated in 2010 (Digby, 2013). This species is nocturnal, and both sexes call intermittently throughout the night, sometimes in male-female duets. The calls consist of many repeated syllables slowly rising in pitch, with fundamental frequency around $2-3 \mathrm{kHz}$ for males and $1-2 \mathrm{kHz}$ for females (Supplementary Figure S7). The number of syllables and exact pitch vary between calls. Further details on LSK acoustics can be found in Digby (2013) and Digby et al. (2014). There are other night callers at the study site, some of which can be confused with the kiwi pukupuku, particularly ruru (Ninox novaeseelandiae, a native owl).

We used data collected by 7 autonomous sound recorders (Song Meter SM2, Wildlife Acoustics Inc., USA) over two nights in 2018, Oct 6 18:00-Oct 7 06:00 and Oct 7 18:00-Oct 8 06:00. The recorders were attached to trees at about $1.5 \mathrm{~m}$ height and spread to cover an area of $600 \times 800 \mathrm{~m}$ (Supplementary Figure S6), with an average spacing of about $300 \mathrm{~m}$, a distance chosen based on rough estimates of call propagation distance. In total, 10080 minutes of audio was recorded across seven recorders.

Data was processed in the AviaNZ software (Marsland et al., 2019). The software was first trained on a small amount of manually annotated data from the same location, collected in September 2018. During this process (see Marsland et al. (2019) for detailed description), the sound is decomposed into a wavelet representation: this basis of compact functions allows the quantification of the energy in different frequency bands (nodes) at discrete time points. The software identifies an optimal set of wavelet nodes for automated call detection. We trained two filters for the kiwi, using 'high' and 'low' calls (fundamental frequency above or below $2 \mathrm{kHz}$, approximately separating males and females) separately. 
The two nights studied were automatically annotated using these filters: as in training, the software reconstructed the signal from selected wavelet nodes and marked putative calls when high energy in the reconstructed signal was detected. All the potential 'calls' detected were reviewed by a human observer to ensure that final output contained only true positives. Detection thresholds were set to obtain $90 \%$ sensitivity on the training data; this is a conservative value, and can be relaxed further to increase the analysis throughput at the risk of missing more calls.

The audio data, automatic recogniser settings used for detection in this paper, and AviaNZ format annotations are available at https ://doi .org/10.5061/ dryad.m70p89d (Oct 6th data) and http://doi .org/10.5281/zenodo. 4057094 (Oct 7th data). The AviaNZ software is available at http://www . avianz.net.

\subsection{Data preparation for SCR}

We converted the call timestamps into binary capture histories across the 7 recorders, to be used in all subsequent SCR models. Firstly, to adjust for recorder clock drift, we measured how many 1 -second blocks had calls present on two neighbouring recorders, and adjusted the clocks in 1-second steps until this overlap was maximised (Supplementary Figure S8). We visually inspected the spectrograms to confirm that most overlaps after adjustment reflected the same call (Supplementary Figure S9). For some pairs of recorders, no clear adjustment was identified, and most overlapping annotations appeared to be different calls. In effect, the 7 recorders were split into 3 non-overlapping groups, ZB-ZI-ZG; ZE; ZA-ZH-ZJ. Annotations separated by $<5$ seconds were merged into a single call, to avoid doublecounting fragmented calls or female responses to male calls. The call timestamps were then converted into a binary capture history for each recorder, formatted as shown in Supplementary Figure S5. Recorder positions were projected to metres easting and northing using Universal Transverse Mercator (UTM) zone 60G.

All statistical analyses were conducted using R. For SCR modelling, we used the ascr package, which is available from https://github.com/b-steve/ascr. All other code used in the analysis is available at https://github.com/jjuod/ acoustic-survey-evaluation.

\subsection{Measuring survey precision on data subsets}

To inspect the relationship between SCR precision and acoustic survey length, we applied SCR models to subsamples of the survey. We fitted acoustic SCR mod- 
els using automatically annotated (human-reviewed) data from the two nights, or subsets of $75 \%, 50 \%, 25 \%$, or $12.5 \%$ of it, by using only annotations within the respective fraction of each hour (e.g., for the $25 \%$ fraction all annotations outside 18:00-18:15, 19:00-19:15, etc., were ignored).

In all SCR models, we used the half-normal function to model detection probability. The area of integration was defined by setting $700 \mathrm{~m}$ radius circles around each recorder, as the estimated detection probability in our setup decayed to $<0.005$ at this distance. As a sensitivity analysis, we also tested a smaller integration mask, restricted to the fenced area of the sanctuary. The main outputs of interest were the call rate density $\hat{D}$, and effective survey area (ESA) in hectares, derived from the detection parameters; the effective detection radius was then calculated as $\sqrt{E S A / \pi}$. The standard errors were calculated using the Hessian or by parametric bootstrap and converted to units of calls/hectare/night. The bootstrap consisted of 200 replicate surveys, generated as in the Simulations section: call locations were drawn from a homogeneous spatial Poisson process, and capture histories were obtained by filtering these. Since the true $D, g_{0}, \sigma$ required for this process are unknown, their estimates $\hat{D}, \hat{g}_{0}, \hat{\sigma}$ from the original model were used instead. The positions of recorders, integration mask and survey length remained the same as in the original model. An SCR model was fitted to each replicate, and density SE estimated as the SD of the bootstrapped estimates $\hat{D}^{* b}$ over the replications $b=1, \ldots, 200$ (Stevenson et al., 2015).

\subsection{Comparison of human and software call detection}

In the next experiment, we used the SCR framework to compare automatic and manual call annotation processes. Manual annotations were made for data from the Oct 6 night, using audio playback and visual inspection of the spectrogram in AviaNZ. The processing was split between two observers, with each annotating approximately half of the dataset. Automatic (human-reviewed) annotations for this night were already obtained as described previously. We fitted two SCR models using either the manual or automatic (reviewed) annotations, and calculated the precision of the final density estimate using bootstrap as above.

For comparison, we also calculated metrics based on agreement between automatic and manual annotations. Using 1 second time resolution, we calculated the numbers of seconds corresponding to true/false negatives/positives (treating manual annotations as the ground truth), and derived sensitivity (or recall, $T P /(T P+$ $F N))$, specificity $(T N /(T N+F P))$, and accuracy $((T P+T N) /(T P+T N+F P+F N))$, 
using standard definitions (Knight et al., 2017; Priyadarshani et al., 2018). Similar calculations were performed using $15 \mathrm{~s}$ windows. In each case, a window that overlaps one or more annotations is considered a positive for the source of annotations.

Finally, we investigated the effect of recorder removal in a similar setup, using data from the night of Oct 6 . We excluded two recorders with the highest levels of noise (ZB and ZH) or worst agreement with human annotations (ZA and ZI). SCR models were then re-fitted using the remaining data, and all metrics recalculated.

\section{$5 \quad$ Kiwi survey results}

\subsection{Precision accounts for changes in sampling effort}

In the first experiment based on real data, we investigated how SCR estimates respond to changes in the amount of data processed.

Automatic detection using a pre-trained wavelet filter in the AviaNZ software identified 5117 minutes of putative calls, of these 3372 were suggested by the 'high' (male) and 1745 by the 'low' (female) call filter. Subsequent human review rejected most of these as false positives, leaving 514 min of calls as the final result from this method of processing (370 from 'high' and 144 from 'low' filter detections). By fitting an SCR model to this dataset, a call density of 2.86 calls per hectare per night was obtained (bootstrapped SE 0.146 calls per hectare per night), based on an effective survey area of 103.4 ha, or equivalently an effective detection radius of $256 \mathrm{~m}$.

When the data was subsampled to contain only a fraction of each hour, the observed precision closely matched a $1 / \sqrt{n}$ relation (Figure 4 ). The asymptotic and bootstrapped standard errors were very similar, as we did not assume any dependency structure in the call locations. This indicates that the sampling effort is reliably captured by the precision measure.

\subsection{Comparing recognition software with human detection}

As a case study demonstrating the usage of the proposed metric, we compare automated call detection by software vs. fully manual processing of recordings. In manual analysis of the first (Oct 6) night of recordings, 915 annotations were made, comprising a total call duration of 232 min (Supplementary Figure S11). Excluding annotations $<5$ seconds, which usually indicate fragments of a single call, 720 calls remained. Processing 42 hours of audio took about 320 minutes of active user 


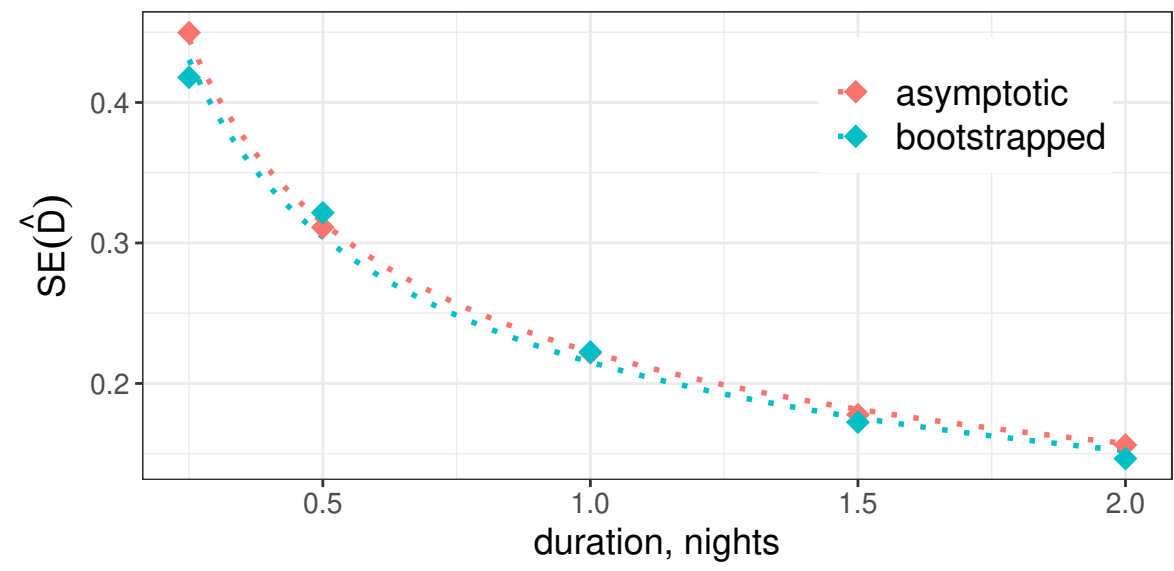

Figure 4: Standard error of estimated call density using a full two-night dataset from a kiwi survey, or subsamples of it. Diamonds are measured SEs (obtained from asymptotic approximations or by bootstrapping), the lines are best-fit curves of the form $y=1 / \sqrt{x}$.

time. The distribution of calls detected by different recorders varied, in particular fewer calls were detected by recorders placed near to a stream (ZE) and on a windy hilltop (ZB).

The automatic processing results (after review) largely matched the manual annotations. Using the human annotation as ground truth, at $1 \mathrm{~s}$ resolution, 3.5\% of the dataset was marked as positives by both methods, and $94.2 \%$ as negatives by both. $1.6 \%$ of the recordings were 'false negatives', i.e., manually annotated as calls, but missed by the automated detection, and the remaining $0.7 \%$ were detected by the automated, but not the manual, processing. Some of these mismatches were due to imprecise annotation boundaries (automatic detection can miss part of a call, or include a few seconds outside the call, both of which are penalised), others represent genuine missed calls.

Using SCR, we obtained similar density estimates for the two processing methods, with slightly lower precision when calls were marked automatically (Table 1, top rows). Density values were well within one SE of each other, and no systematic difference was apparent ( 2.93 vs. 2.89 calls per hectare per night). The automatic processing was worse at detecting faint calls, which in SCR corresponds to a smaller survey area and $8 \%$ larger $\operatorname{Var}(\hat{D})$. In terms of the detection function, human processing resulted in $61 \%$ detection probability at $200 \mathrm{~m}$, in contrast to $54 \%$ when processing automatically. Changing the integration mask to match the sanctuary shape had little influence on the estimated detection parameters, and the precision remained similar (SE 0.258 for the manual annotations, and 0.291 or 13\% worse for the automatic ones), while the estimated density increased to reflect the 
smaller area (3.58 and 3.38 calls/ha/night for the manual and automatic methods, respectively).

\begin{tabular}{l|lllll}
\hline $\begin{array}{l}\text { Recorders / an- } \\
\text { notations }\end{array}$ & $\begin{array}{l}\text { Density } \pm \text { SE, } \\
\text { calls/ha/night }\end{array}$ & ESA, ha & Sensitivity & Specificity & Accuracy \\
\hline $\begin{array}{l}\text { all / manual } \\
\text { all / auto }\end{array}$ & $2.93 \pm 0.215$ & 114.3 & & & \\
\hline -ZA -ZI / manual & $3.89 \pm 0.227$ & 99.3 & $68.90 \%$ & $99.26 \%$ & $97.71 \%$ \\
-ZA -ZI / auto & $3.91 \pm 0.600$ & 57.2 & $69.59 \%$ & $99.40 \%$ & $98.10 \%$ \\
\hline -ZB -ZH / manual & $3.57 \pm 0.307$ & 82.9 & & & \\
-ZB -ZH / auto & $3.39 \pm 0.340$ & 73.2 & $70.00 \%$ & $99.23 \%$ & $97.74 \%$ \\
\hline
\end{tabular}

Table 1: Changes in concordance and SCR measures when using different sets of recorders (all 7, excluding ZA and ZI, or excluding ZB and ZH) or different detection methods (manual or automatic). SCR models were fitted to Oct 6 night data, and estimated call density and effective survey area (ESA) were calculated. Standard error is the standard deviation of bootstrapped density estimates. Sensitivity, specificity, accuracy obtained by comparing manual and automatic annotations over 1 second blocks.

However, manual processing was considerably more labour-intensive. Review of software outputs took 132 minutes, making the automated processing with review 2.4 times faster (in user time) than the fully manual workflow. Equivalently, given fixed total effort, 2.4 times more data could be processed in the automated workflow, with almost all user time spent on the review of the detections. Based on Figure 4, this would correspond to a $60 \%$ gain in precision, far outweighing the $8 \%$ loss due to missed calls.

\subsection{Standard agreement measures do not reflect optimal design choices}

To provide direct evidence of the shortcomings of standard concordance measures, we show that the match between manual and automatic annotations can be improved by reducing the dataset. When using data from all 7 recorders, the overall sensitivity of the automatic detection method was $68.9 \%$, specificity $99.3 \%$, and accuracy $97.7 \%$ (Table 1, Supplementary Table S2). Recorder ZA had the lowest sensitivity (59.7\%), and ZI the lowest accuracy (96.2\%); on these grounds, data from these two recorders was excluded, and all measures recalculated using data from the 5 remaining recorders. Despite the obvious loss of information, all agreement measures increased: sensitivity to $69.6 \%$, specificity to $99.4 \%$, accuracy to 
98.1\%. Similarly, the ZB and ZH recordings were found to have a great deal of wind noise in them. Excluding these instead gave an even larger increase in sensitivity and almost no change in specificity or accuracy. In contrast, data loss decreased the SCR estimate precision in both cases: standard errors were increased by $40-75 \%$, while the density estimate itself was increased by about $20 \%$.

We also note that the agreement measures depended on the resolution at which presence/absence was determined. Using larger windows (15 s instead of $1 \mathrm{~s}$ ), we obtained considerably higher sensitivity for every recorder combination tested (increase to around 75\% from 69\%), with specificity still remaining above $99 \%$ (Supplementary Table S3). A drop in accuracy to $95.4 \%$ was observed, as would be expected when the fraction of positives in the ground truth (or true species prevalence) increases.

\section{Discussion}

Many studies over the last two decades have suggested improvements to the methodology of performing and analysing acoustic surveys for conservation purposes. In this paper we have highlighted some of the issues that can lead to incorrect decisions when evaluating such improvements: (1) optimal design in certain models strongly depends on unknown parameters, such as the true occupancy, and (2) the success of a single stage of the survey process does not necessarily reflect the overall performance.

The former issue is known in the occupancy modelling literature (Mackenzie and Royle, 2005; Gálvez et al., 2016), but the latter has received less attention. For example, call recognition is mainly evaluated using agreement metrics, and even though some problems stemming from them have been discussed (Knight et al., 2017; Priyadarshani et al., 2018; Sofaer et al., 2019), no alternatives have been proposed. In the main comparison presented here - manual versus automatic call detection - we have shown that the standard metrics rate the detection software poorly, since they do not account for the gain in processing speed, nor accurately reflect the importance of missed detections. We also observed that detrimental changes to the survey (deleting large portion of data in this case) can produce no effect, or even appear as improvements, on a single stage of the processing pipeline. In addition, the standard performance metrics depend on the measurement resolution, as seen in the literature, e.g., Metcalf et al. (2019).

We propose that these issues could be alleviated by using the precision of the 
SCR density estimate as a metric for evaluating acoustic survey design. As it has a close relationship with the inferential power, this metric corresponds to the desired ecological outputs at the application level. Our application demonstrates that this approach properly accounts for changes in effective sample size, and correctly identifies that automatic processing provides an overall improvement to survey efficiency.

To conduct such a comparison in practice, researchers would identify a set of possible designs to be compared, and then estimate the (occupancy or SCR) precision obtained with each design to identify the more efficient one. Ideally, this would use a real pilot dataset corresponding to each option. This is easier when the compared options are differences in data processing, as in our kiwi study; to compare different hardware, for example, two sets of recordings from the same location would be needed. The data then would be annotated, SCR or occupancy model applied, and the resulting standard errors compared; packages that implement either analysis, such as ascr (Stevenson et al., 2015) or unmarked (Fiske and Chandler, 2011), also include bootstrap routines for calculating SEs even with complicated designs. Alternatively, the researcher can simulate detection histories corresponding to each of the design options and then analyse them by occupancy or SCR, or even calculate the precision directly with the approximation formulas (Mackenzie and Royle, 2005; Efford and Boulanger, 2019). Still, these cases will require some experimental data to obtain the detection parameters, and assumed values for true $D$ or $\Psi$. While the evaluation process is similar with either SCR or occupancy, we observed here that in the latter case, optimal choices depend on the unmeasured parameters, and thus occupancy cannot be directly applied to identify general improvements such as better call detectors.

Even for SCR, some knowledge of the target species ecology is still required. Our metric does not evaluate whether or not the model was specified correctly. For example, spatial dependency in detections across recorders or territorial behaviour will impact the true variance of the density estimator, but will not be reflected in the SE if it is calculated from a simpler model. This is still an issue even if the precision is estimated by study-level bootstrap, as it is still model-based, although it can be used to test the effects of suspected deviations. Expected call propagation distance is needed for choosing the spacing between detectors, as the estimates will be biased if the captures and recaptures of calls are very unbalanced. There is some evidence that SCR is robust to moderate deviations in this regard (Sun et al., 2014), and pilot tests with playback should be sufficient to establish appropriate 
spatial design.

Additionally, knowledge of the calling behaviour is needed to convert the density of calls into actual population counts. The simplest approach is to assume a constant call rate, so that $D_{a}$ would be proportional to $D$, but even then its value for a specific survey is rarely known: for kiwi, like many other species, calling is correlated with many factors such as season, temperature, and light levels (Keast, 1994; Digby, 2013; Watson et al., 2016). Current monitoring protocols further rely on these factors being equal in all surveys compared, so population trends are measured by changes in cue density or count, e.g., Colbourne and Digby (2016). Alternatively, Stevenson et al. (2021) recently proposed an extension of the SCR model that simultaneously estimates the survey-specific call rate, by assuming that individuals do not move between calls. Within-survey variation in the environmental factors may also be a problem. If measured, the factors can be set as covariates on an inhomogeneous density $D(\boldsymbol{x})$, thus in effect allowing spatial variations in call rate. Ideally, this issue can be largely bypassed if recognition of individuals from their calls becomes possible.

More generally, practitioner and analyst expertise is still required to ensure appropriate match between data and processing, which is not easily captured by simple summary metrics. Behavioural knowledge can be applied to define cue units (whether for manual annotation or automatic detector training) in a way that helps satisfy various SCR assumptions, such as removing responses from call duets (Dawson and Efford, 2009). Choice of the time periods for recording and processing will influence the interpretation of the data, and also determine more appropriate modelling; e.g. peak calling times may lead to higher apparent precision, and may represent the population more completely, but may be difficult to time across years (Colbourne and Digby, 2016). Ignoring these issues may lead to bias in the SCR models, which will only be detected if specifically tested for in simulations. However, these problems are not specific to SCR - e.g. temporal or spatial variations would appear as varying availability $p_{a}$ in the occupancy models, and appropriate adjustments would still be required.

As an indication of overall validity of call rates estimated here, our estimates can be roughly compared to the literature. Approximate rates can be obtained from the data in Colbourne and Digby (2016) to be between 0.64-2.8 calls/night for six sites with two other kiwi species. Assuming kiwi density in our study area was about 1 individual per hectare (Digby, 2013), a reasonably similar call rate of 2.9 calls/night was obtained. Our estimates of the detection parameters also 
match a previous playback experiment for the same kiwi species, which reported effective detection radius of about 300-400 m, or an area of about 25 hectares for a single recorder (Digby et al., 2013). We emphasise that this comparison is only for illustrative purposes, and typically longer observation periods are needed to reliably establish a call rate, as weather conditions and seasonal effects can cause large variation in nightly behaviour.

Although we demonstrated the issues in evaluating two particular types of survey - occupancy models and grid-based SCR - various other study designs are common in ecoacoustics, and can be evaluated using similar ideas, providing they result in a single estimate of abundance and its precision. Soundscape studies (Pijanowski et al., 2011; Sueur et al., 2014), for example, use acoustic indices to characterise a community directly, without estimating the abundance of each species. Recently, Bradfer-Lawrence et al. (2019) showed how a similar framework, focusing on the overall precision of the index estimation, can be used to optimise the design of soundscape studies. Even if we consider only single-species, single-population surveys using autonomous recorders, different ways to use the recorders are possible, leading to different design issues.

The overlapping grid setup used in this study is uncommon: Sugai et al. (2020) found that $71 \%$ of passive acoustic monitoring studies used only a single recorder per site. However, many different designs, including single-recorder ones, can be expressed as special cases of SCR, providing that some source of information about the detection function is available, such as the estimated distance to each cue for distance sampling (Borchers et al., 2015). As an example, Sebastián-González et al. (2018) estimated the relationship between distance and recorded power of a bird call in a separate experiment, and then used this function to allow distance sampling from single-recorder sites. Another option is to calibrate the detection function using playback (Darras et al., 2016; Hagens et al., 2018), although this is not trivial because it requires knowing the true loudness at which the animal vocalises naturally. In any case, the framework presented here can be used with such surveys, and even allows the user to compare between the configurations, e.g., to evaluate the cost-benefit tradeoff of using a recorder grid. The latter task would require measuring the uncertainty in the pilot estimates of the detection function; incorporating that into the current approach could be a useful future development.

Another development that could greatly extend the use of this framework is quantifying the effects of false positive detections. These are unavoidable when using automated call recognisers (Knight et al., 2017; Priyadarshani et al., 2018), but 
violate SCR assumptions with unknown consequences. In this study, all detections were manually reviewed, so that instead of trying to optimise the balance of false negatives and false positives, we optimise false negatives vs. the review effort - a tradeoff that can be solved with the proposed precision metric. For some species, even expert identification may be unreliable (Mortimer and Greene, 2017), and estimating the effects of misdetection is necessary before the proposed framework can be applied. In the future, such measures could even open up the possibility of fully automated population monitoring pipelines. Further experimental work is also needed to establish the validity of various aspects of SCR: true assignment of calls to individuals, for example, could be used to verify the overall population estimates and evaluate if the current calling behaviour models are sufficiently realistic.

Occupancy studies avoid many of these issues and ultimately may be easier to conduct in practice. The popularity and ease of use of the occupancy framework also means that more data is available for historical comparisons, and it will likely remain a common approach in monitoring. However, guiding occupancy studies by their precision relies on knowing study-specific parameters such as the true occupancy, which are typically unavailable at the onset of a study. In contrast, final precision of SCR allows the experimenter to identify universally optimal design choices, which is necessary for developing methods such as call detection software. The SCR framework can be readily extended to incorporate other types of information available to the researcher, e.g., time of arrival, telemetry data, or spatial covariates (Borchers et al., 2015; Linden et al., 2018), and applied to any vocalising species. Because of this flexibility, a large variety of management decisions can be evaluated following the approach presented here. We hope that the established SCR methods, combined with our baseline results and field dataset, which allows the testing of new analysis methods on standardised data, will provide a consistent way to evaluate new developments in acoustic survey design and analysis.

\section{Bibliography}

Acevedo, M. A., Corrada-Bravo, C. J., Corrada-Bravo, H., Villanueva-Rivera, L. J. and Aide, T. M. (2009) Automated classification of bird and amphibian calls using machine learning: A comparison of methods. Ecological Informatics, 4, 206-214.

Atkinson, A. C. and Donev, A. N. (1992) Optimum Experimental Designs. Oxford University Press.

Bai, Y., Song, P. X.-K. and Raghunathan, T. E. (2012) Joint composite estimating functions 
in spatiotemporal models. Journal of the Royal Statistical Society: Series B (Statistical Methodology), 74, 799-824.

Blumstein, D. T., Mennill, D. J., Clemins, P., Girod, L., Yao, K., Patricelli, G., Deppe, J. L., Krakauer, A. H., Clark, C., Cortopassi, K. A., Hanser, S. F., McCowan, B., Ali, A. M. and Kirschel, A. N. G. (2011) Acoustic monitoring in terrestrial environments using microphone arrays: applications, technological considerations and prospectus. Journal of Applied Ecology, 48, 758-767.

Borchers, D. L., Stevenson, B. C., Kidney, D., Thomas, L. and Marques, T. A. (2015) A Unifying Model for Capture-Recapture and Distance Sampling Surveys of Wildlife Populations. Journal of the American Statistical Association, 110, 195-204.

Boulanger, J., Nielsen, S. E. and Stenhouse, G. B. (2018) Using spatial mark-recapture for conservation monitoring of grizzly bear populations in Alberta. Scientific Reports, 8, 5204.

Bradfer-Lawrence, T., Gardner, N., Bunnefeld, L., Bunnefeld, N., Willis, S. G. and Dent, D. H. (2019) Guidelines for the use of acoustic indices in environmental research. Methods in Ecology and Evolution, 10, 1796-1807.

Brandes, T. S. (2008) Automated sound recording and analysis techniques for bird surveys and conservation. Bird Conservation International, 18, S163-S173.

Clement, M. J. (2016) Designing occupancy studies when false-positive detections occur. Methods in Ecology and Evolution, 7, 1538-1547.

Colbourne, R. and Digby, A. (2016) Call rate behaviour of brown kiwi (Apteryx mantelli) and great spotted kiwi (A. haastii) in relation to temporal and environmental parameters. DOC Research and Development Series, 22.

Collier, T. C., Kirschel, A. N. G. and Taylor, C. E. (2010) Acoustic localization of antbirds in a Mexican rainforest using a wireless sensor network. The Journal of the Acoustical Society of America, 128, 182-189.

Connor, E. F., Li, S. and Li, S. (2012) Automating identification of avian vocalizations using time-frequency information extracted from the Gabor transform. The Journal of the Acoustical Society of America, 132, 507-517.

Cook, A. and Hartley, S. (2018) Efficient sampling of avian acoustic recordings: intermittent subsamples improve estimates of single species prevalence and total species richness. Avian Conservation and Ecology, 13.

Darras, K., Batáry, P., Furnas, B. J., Grass, I., Mulyani, Y. A. and Tscharntke, T. (2019) Autonomous sound recording outperforms human observation for sampling birds: a systematic map and user guide. Ecological Applications, 29.

Darras, K., Pütz, P., Fahrurrozi, Rembold, K. and Tscharntke, T. (2016) Measuring sound detection spaces for acoustic animal sampling and monitoring. Biological Conservation, 201, 29-37. 
Dawson, D. K. and Efford, M. G. (2009) Bird population density estimated from acoustic signals. Journal of Applied Ecology, 46, 1201-1209.

Digby, A. (2013) Whistling in the Dark: an Acoustic Study of Little Spotted Kiwi. Ph.D. thesis, Victoria University of Wellington.

Digby, A., Bell, B. D. and Teal, P. D. (2014) Vocal individuality of little spotted kiwi (Apteryx owenii). Emu - Austral Ornithology, 114, 326-336.

Digby, A., Towsey, M., Bell, B. D. and Teal, P. D. (2013) A practical comparison of manual and autonomous methods for acoustic monitoring. Methods in Ecology and Evolution, 4, 675-683.

Efford, M. (2004) Density estimation in live-trapping studies. Oikos, 106, 598-610.

Efford, M. G. and Boulanger, J. (2019) Fast evaluation of study designs for spatially explicit capture-recapture. Methods in Ecology and Evolution, 10, 1529-1535.

Efford, M. G., Dawson, D. K. and Borchers, D. L. (2009) Population density estimated from locations of individuals on a passive detector array. Ecology, 90, 2676-2682.

Fiske, I. and Chandler, R. (2011) unmarked: An R package for fitting hierarchical models of wildlife occurrence and abundance. Journal of Statistical Software, 43.

Gálvez, N., Guillera-Arroita, G., Morgan, B. J. and Davies, Z. G. (2016) Cost-efficient effort allocation for camera-trap occupancy surveys of mammals. Biological Conservation, 204, 350-359.

Gibb, R., Browning, E., Glover-Kapfer, P. and Jones, K. E. (2018) Emerging opportunities and challenges for passive acoustics in ecological assessment and monitoring. Methods in Ecology and Evolution, 10, 169-185.

Hagens, S. V., Rendall, A. R. and Whisson, D. A. (2018) Passive acoustic surveys for predicting species' distributions: Optimising detection probability. PLOS ONE, 13, e0199396.

Keast, A. (1994) Temporal vocalisation patterns in members of a eucalypt forest bird community: the effects of weather on song production. Emu - Austral Ornithology, 94, 172180.

Knight, E., Hannah, K., Foley, G., Scott, C., Brigham, R. and Bayne, E. (2017) Recommendations for acoustic recognizer performance assessment with application to five common automated signal recognition programs. Avian Conservation and Ecology, 12.

Lemckert, Penman and Mahony (2005) A cost-benefit analysis of automated call recorders. Applied Herpetology, 2, 389-400.

Linden, D. W., Sirén, A. P. K. and Pekins, P. J. (2018) Integrating telemetry data into spatial capture-recapture modifies inferences on multi-scale resource selection. Ecosphere, 9, e02203.

MacKenzie, D. I., Nichols, J. D., Lachman, G. B., Droege, S., Royle, J. A. and Langtimm, C. A. (2002) Estimating site occupancy rates when detection probabilities are less than one. Ecology, 83, 2248-2255. 
Mackenzie, D. I. and Royle, J. A. (2005) Designing occupancy studies: general advice and allocating survey effort. Journal of Applied Ecology, 42, 1105-1114.

Marsland, S., Priyadarshani, N., Juodakis, J. and Castro, I. (2019) AviaNZ: A future-proofed program for annotation and recognition of animal sounds in long-time field recordings. Methods in Ecology and Evolution, 10, 1189-1195.

Metcalf, O. C., Ewen, J. G., McCready, M., Williams, E. M. and Rowcliffe, J. M. (2019) A novel method for using ecoacoustics to monitor post-translocation behaviour in an endangered passerine. Methods in Ecology and Evolution, 10, 626-636.

Mortimer, J. and Greene, T. (2017) Investigating bird call identification uncertainty using data from processed audio recordings. New Zealand Journal of Ecology, 41.

Pérez-Granados, C., Bota, G., Giralt, D., Albarracín, J. and Traba, J. (2019) Costeffectiveness assessment of five audio recording systems for wildlife monitoring: Differences between recording distances and singing direction. Ardeola, 66, 311.

Pérez-Granados, C., Bota, G., Giralt, D. and Traba, J. (2018) A cost-effective protocol for monitoring birds using autonomous recording units: a case study with a night-time singing passerine. Bird Study, 65, 338-345.

Pijanowski, B. C., Villanueva-Rivera, L. J., Dumyahn, S. L., Farina, A., Krause, B. L., Napoletano, B. M., Gage, S. H. and Pieretti, N. (2011) Soundscape ecology: The science of sound in the landscape. BioScience, 61, 203-216.

Potamitis, I., Ntalampiras, S., Jahn, O. and Riede, K. (2014) Automatic bird sound detection in long real-field recordings: Applications and tools. Applied Acoustics, 80, 1-9.

Priyadarshani, N., Marsland, S. and Castro, I. (2018) Automated birdsong recognition in complex acoustic environments: a review. Journal of Avian Biology, 49, jav-01447.

Priyadarshani, N., Marsland, S., Castro, I. and Punchihewa, A. (2016) Birdsong Denoising Using Wavelets. PLOS ONE, 11, e0146790.

Reich, B. J. and Gardner, B. (2014) A spatial capture-recapture model for territorial species. Environmetrics, 25, 630-637.

Rempel, R. S., Francis, C. M., Robinson, J. N. and Campbell, M. (2013) Comparison of audio recording system performance for detecting and monitoring songbirds. Journal of Field Ornithology, 84, 86-97.

Riddle, J. D., Stanislav, S. J., Pollock, K. H., Moorman, C. E. and Perkins, F. S. (2010) Separating components of the detection process with combined methods: An example with northern bobwhite. Journal of Wildlife Management, 74, 1319-1325.

Sebastián-González, E., Camp, R. J., Tanimoto, A. M., de Oliveira, P. M., Lima, B. B., Marques, T. A. and Hart, P. J. (2018) Density estimation of sound-producing terrestrial animals using single automatic acoustic recorders and distance sampling. Avian Conservation and Ecology, 13.

Shonfield, J. and Bayne, E. (2017) Autonomous recording units in avian ecological research: current use and future applications. Avian Conservation and Ecology, 12. 
Sofaer, H. R., Hoeting, J. A. and Jarnevich, C. S. (2019) The area under the precision-recall curve as a performance metric for rare binary events. Methods in Ecology and Evolution, 10, 565-577.

Stevenson, B. C., Borchers, D. L., Altwegg, R., Swift, R. J., Gillespie, D. M. and Measey, G. J. (2015) A general framework for animal density estimation from acoustic detections across a fixed microphone array. Methods in Ecology and Evolution, 6, 38-48.

Stevenson, B. C., van Dam-Bates, P., Young, C. K. Y. and Measey, J. (2021) A spatial capture-recapture model to estimate call rate and population density from passive acoustic surveys. Methods in Ecology and Evolution, 12, 432-442.

Stowell, D., Wood, M. D., Pamuła, H., Stylianou, Y. and Glotin, H. (2018) Automatic acoustic detection of birds through deep learning: The first bird audio detection challenge. Methods in Ecology and Evolution, 10, 368-380.

Sueur, J., Farina, A., Gasc, A., Pieretti, N. and Pavoine, S. (2014) Acoustic indices for biodiversity assessment and landscape investigation. Acta Acustica united with Acustica, 100, 772-781.

Sugai, L. S. M., Desjonquères, C., Silva, T. S. F. and Llusia, D. (2020) A roadmap for survey designs in terrestrial acoustic monitoring. Remote Sensing in Ecology and Conservation, 6, 220-235.

Sugai, L. S. M., Silva, T. S. F., Ribeiro, J. W. and Llusia, D. (2018) Terrestrial passive acoustic monitoring: Review and perspectives. BioScience, 69, 15-25.

Sun, C. C., Fuller, A. K. and Royle, J. A. (2014) Trap configuration and spacing influences parameter estimates in spatial capture-recapture models. PLoS ONE, 9, e88025.

Swiston, K. A. and Mennill, D. J. (2009) Comparison of manual and automated methods for identifying target sounds in audio recordings of pileated, pale-billed, and putative ivory-billed woodpeckers. Journal of Field Ornithology, 80, 42-50.

Towsey, M., Planitz, B., Nantes, A., Wimmer, J. and Roe, P. (2012) A toolbox for animal call recognition. Bioacoustics, 21, 107-125.

Varin, C., Reid, N. and Firth, D. (2011) An overview of composite likelihood methods. Statistica Sinica, 21, 5-42.

Watson, M. J., Wilson, D. R. and Mennill, D. J. (2016) Anthropogenic light is associated with increased vocal activity by nocturnally migrating birds. The Condor, 118, 338-344.

Williams, E. M., O’Donnell, C. F. J. and Armstrong, D. P. (2018) Cost-benefit analysis of acoustic recorders as a solution to sampling challenges experienced monitoring cryptic species. Ecology and Evolution, 8, 6839-6848.

Yip, D. A., Leston, L., Bayne, E. M., Sólymos, P. and Grover, A. (2017) Experimentally derived detection distances from audio recordings and human observers enable integrated analysis of point count data. Avian Conservation and Ecology, 12.

Zhao, Z., Ma, T. F., Ng, W. L. and Yau, C. Y. (2019) A composite likelihood-based approach for change-point detection in spatio-temporal process. arXiv preprint: 1904.06340. 


\section{Supplementary figures and tables}

captures:

recorders

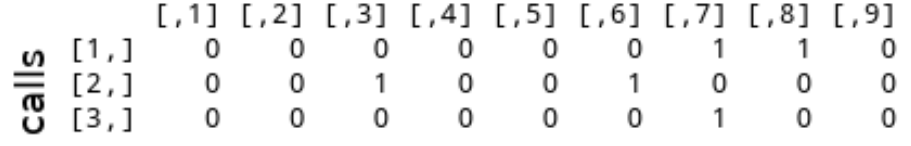

\section{locations:}

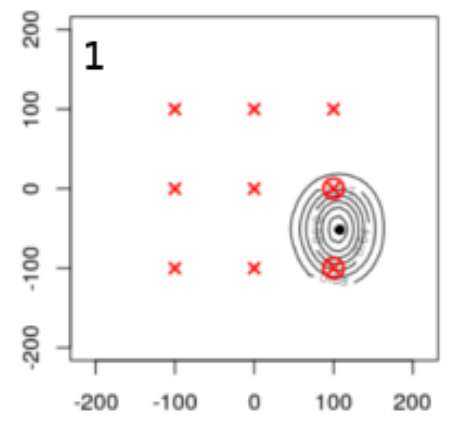

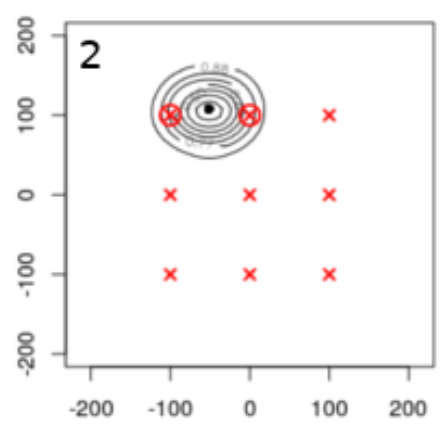

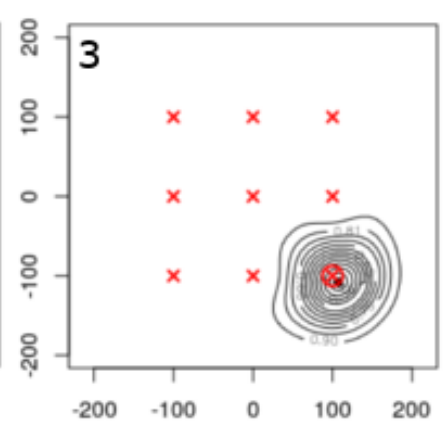

Figure S5: Example of simulated inputs and outputs of an SCR model. Capture history is a binary matrix of calls $\times$ recorders, assuming a single occasion (first 3 calls are shown here). "Traps" are positions of 9 acoustic recorders, using relative units from the grid center in this example. The images show, for each of the 3 calls, the grid of all recorders (crosses), recorders that detected this call (circled), and post-hoc estimate of the call location (black dots and probability contours). 


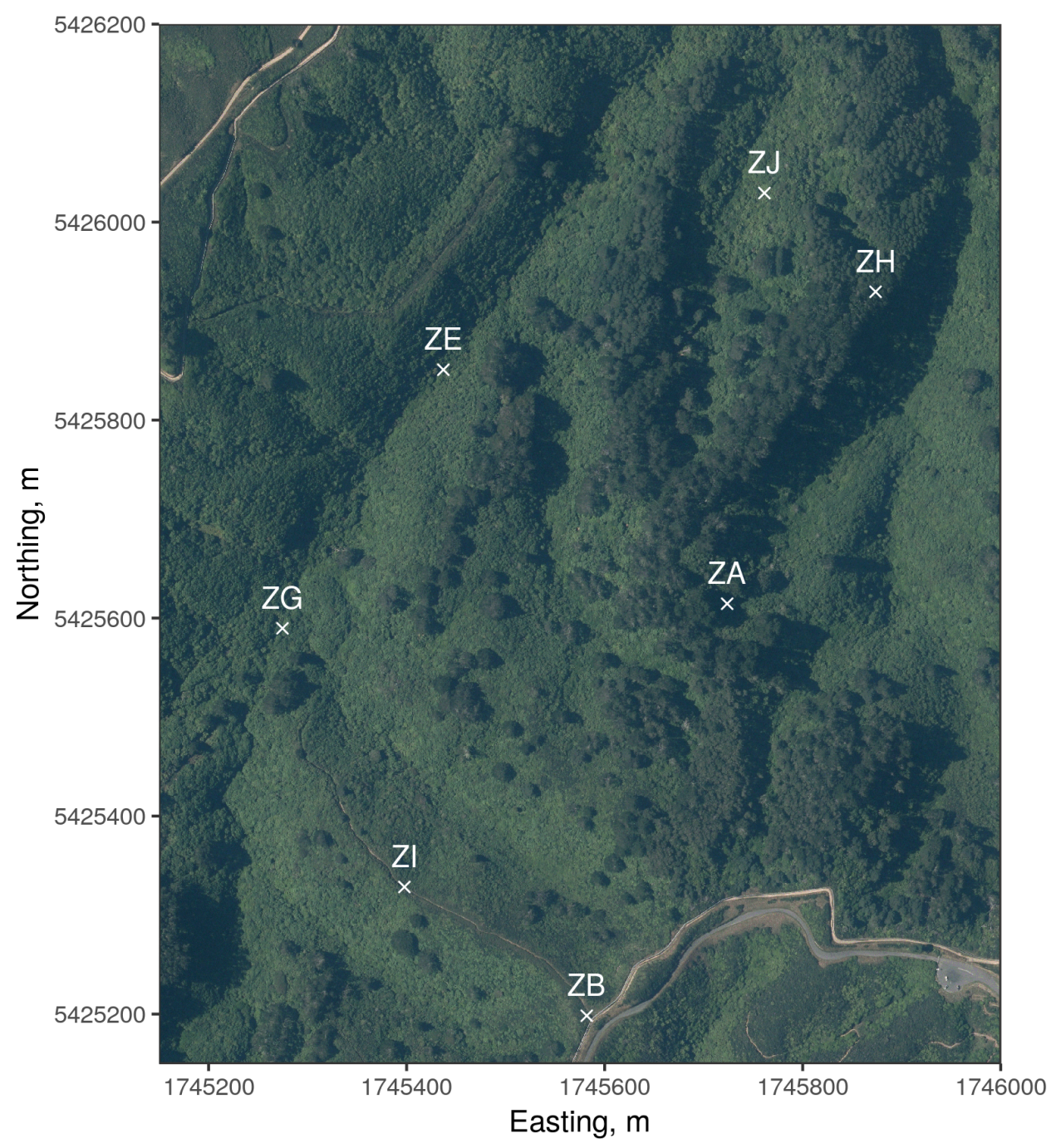

Figure S6: Positions of the 7 recorders used in this study within Zealandia wildlife sanctuary in Wellington, New Zealand. Northing and easting coordinates obtained by UTM projection, zone 60 South. Satellite photography sourced from the LINZ Data Service. 


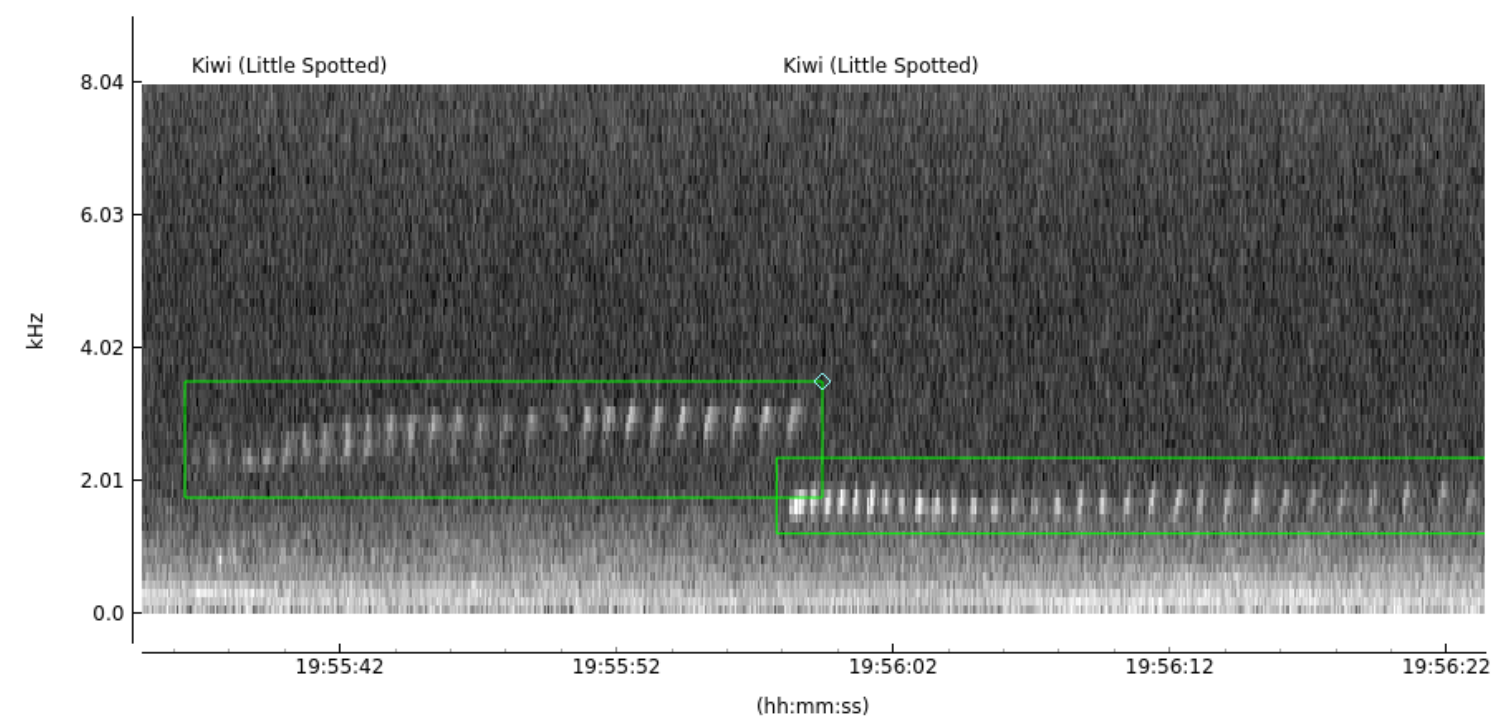

Figure S7: Spectrogram representation of two kiwi pukupuku calls - male followed by female. Spectrogram and annotations produced using the AviaNZ software.

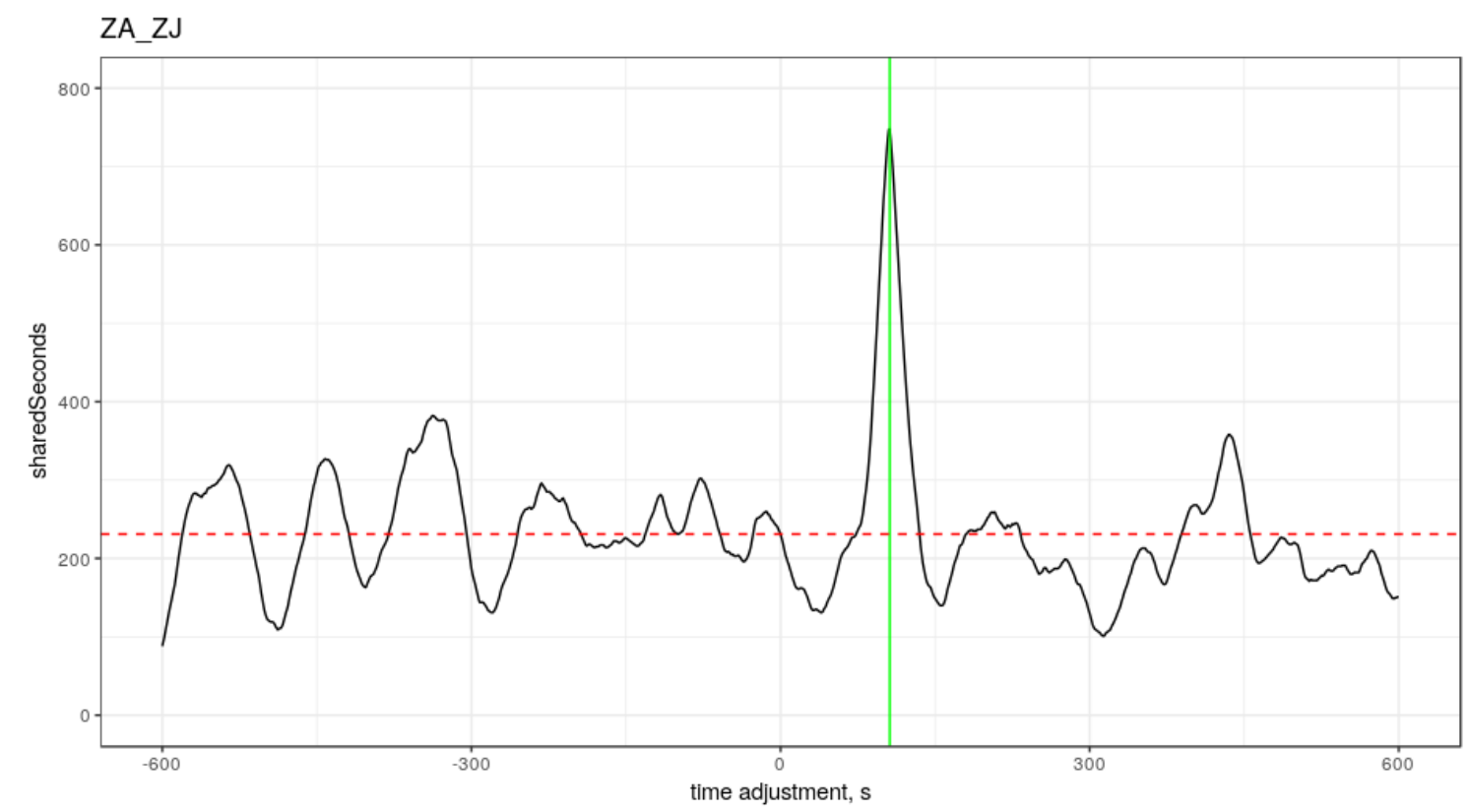

Figure S8: Total duration of overlapping calls across ZA-ZJ pair of recorders for various clock shifts. ZA clock is shifted between $600 \mathrm{~s}$ lag (left) to $600 \mathrm{~s}$ lead (right). Clear peak is identified, marked with a green line. Call overlap without clock adjustment (i.e. at adjustment 0 ) is marked with a red dashed line. 


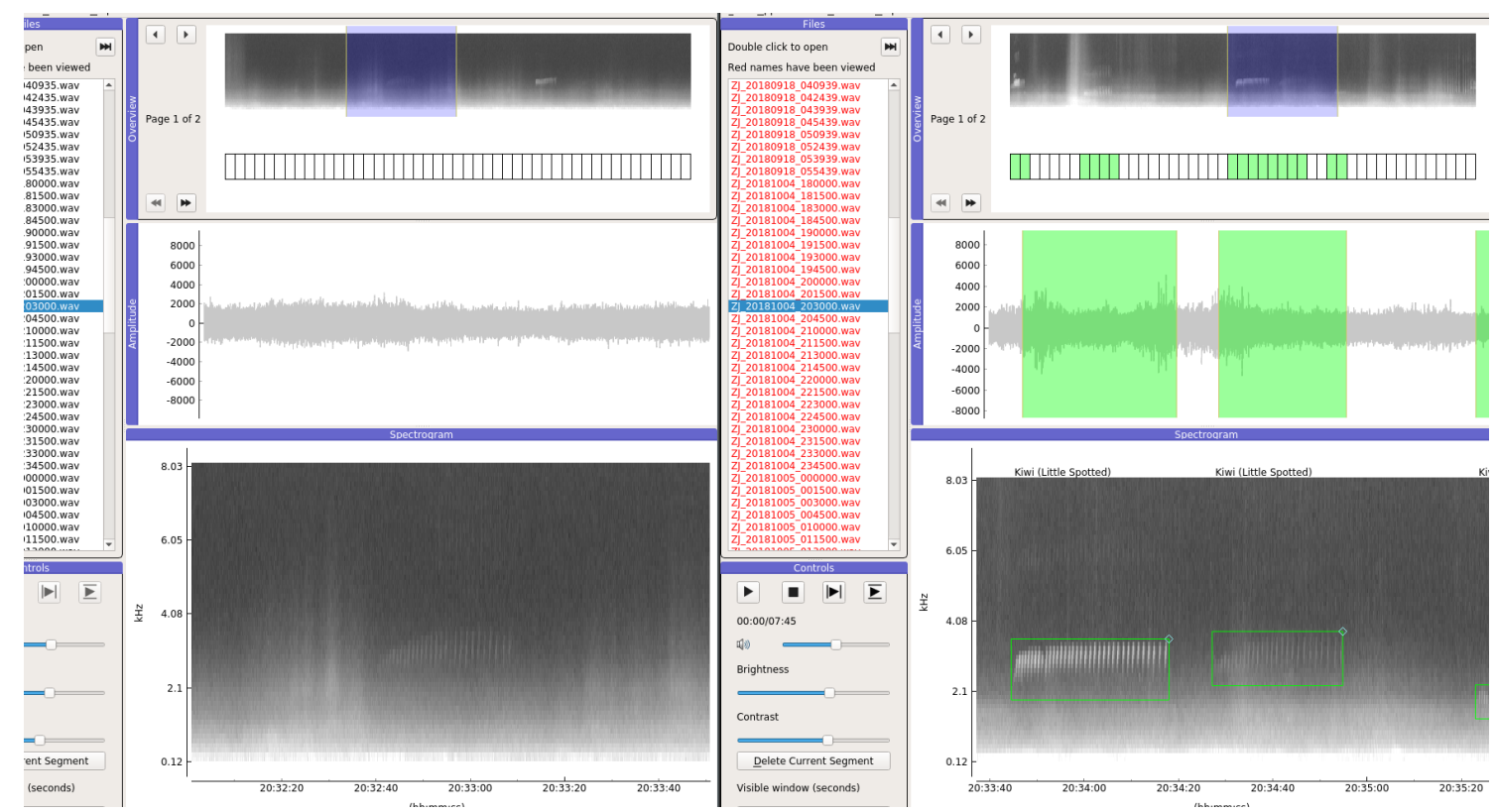

Figure S9: Example of overlapping calls as seen in AviaNZ. Spectrogram positions aligned based on identified clock lag (about $100 \mathrm{~s}$ lag on ZA). Both ZA (left) and ZJ (right) recorders capture the same call around 20:34:40 on ZJ. Call at 20:34:00 appears loud on ZJ, but not detected on ZA, indicating that it is close to the $\mathrm{ZJ}$ recorder.
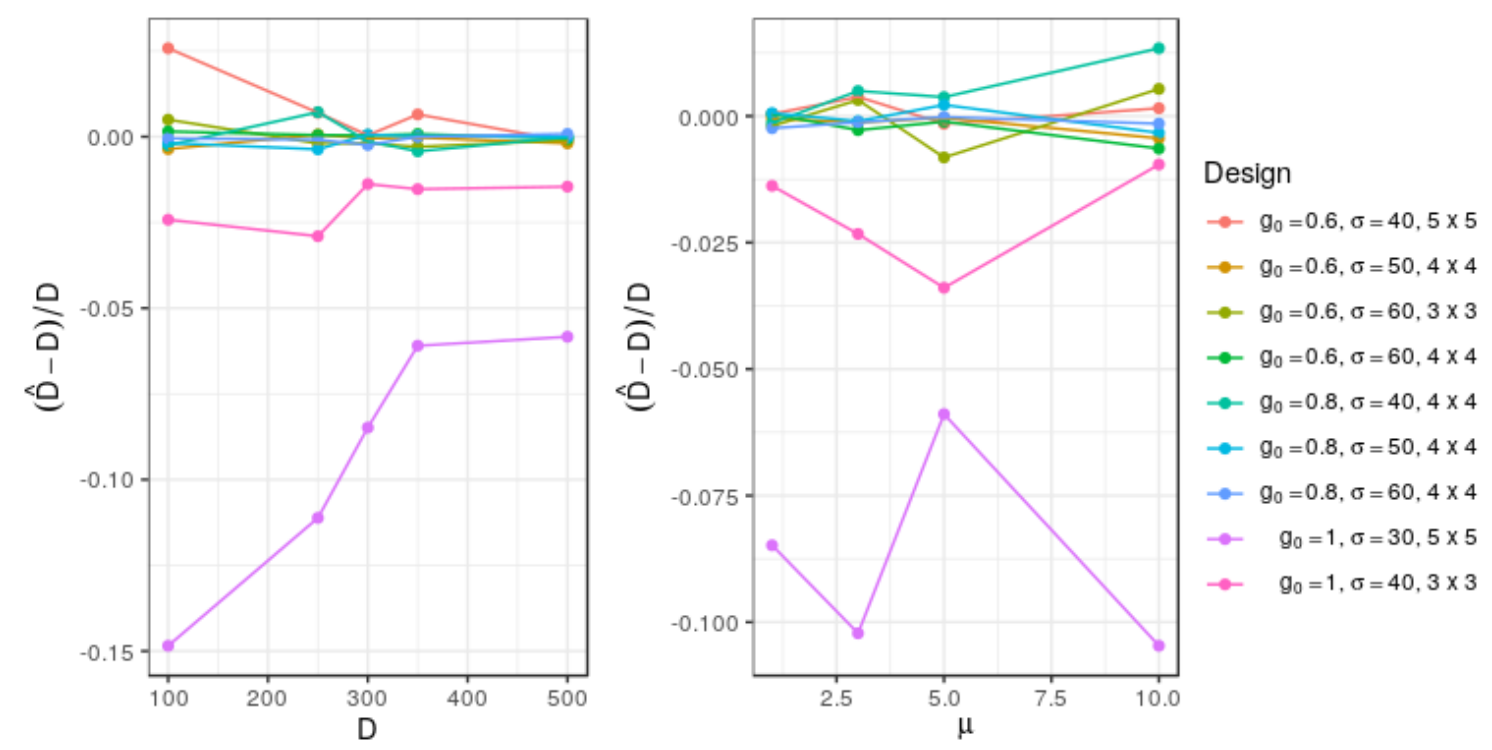

Figure S10: Relative bias of SCR estimates from different study designs, obtained from simulations. Call locations were simulated to be independent with density $D$ (left), or dependent, replicated at the same location $\mu$ times, with overall density of 300 (right). For each design setting (colors), 100 datasets were simulated. 


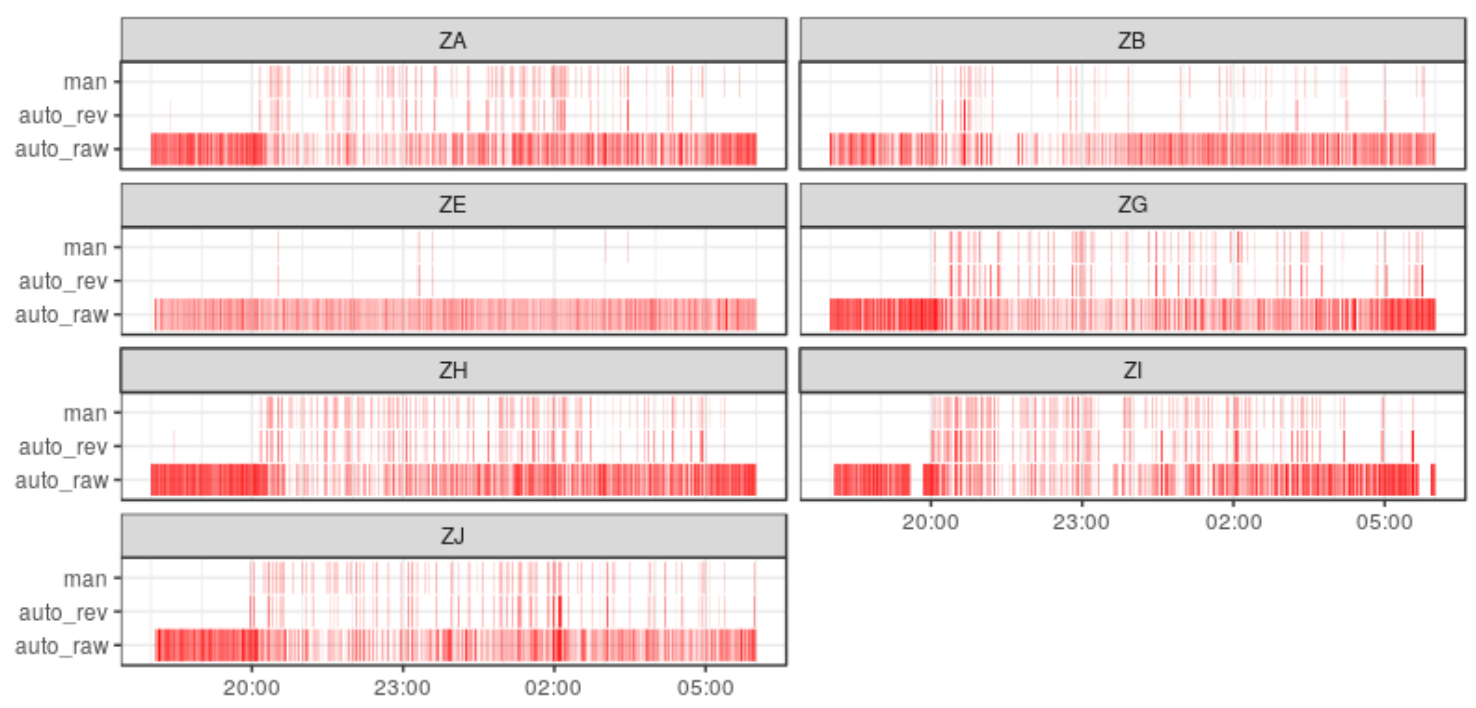

Figure S11: Automated annotations before review (auto_raw), automated annotations after review (auto_rev), and manual annotations (man) for Oct 6 night across 7 recorders. Red segments correspond to sections of audio marked as calls.

\begin{tabular}{lllll}
\hline Recorder & Sensitivity & Precision & Specificity & Accuracy \\
\hline ZA & $59.67 \%$ & $90.38 \%$ & $99.60 \%$ & $97.26 \%$ \\
ZB & $62.07 \%$ & $79.25 \%$ & $99.59 \%$ & $98.68 \%$ \\
ZE & $65.93 \%$ & $91.75 \%$ & $99.98 \%$ & $99.88 \%$ \\
ZG & $72.85 \%$ & $82.97 \%$ & $99.18 \%$ & $97.80 \%$ \\
ZH & $67.42 \%$ & $86.48 \%$ & $99.10 \%$ & $96.61 \%$ \\
ZI & $73.63 \%$ & $78.95 \%$ & $98.23 \%$ & $96.20 \%$ \\
ZJ & $72.91 \%$ & $83.16 \%$ & $99.08 \%$ & $97.53 \%$ \\
\hline
\end{tabular}

Table S2: Concordance between manual and automatic (human-reviewed) annotations for Oct 6 18:00-Oct 7 06:00 for the 7 recorders. Concordance measures (sensitivity, false positive rate, precision, specificity, and accuracy) calculated following standard definitions, after discretizing annotations into 1 second intervals.

\begin{tabular}{llll}
\hline Recorders / annotations & Sensitivity & Specificity & Accuracy \\
\hline all / manual & $74.87 \%$ & $99.09 \%$ & $95.38 \%$ \\
-ZA -ZI / manual & $74.79 \%$ & $99.23 \%$ & $96.06 \%$ \\
-ZB -ZH / manual & $76.84 \%$ & $99.02 \%$ & $95.67 \%$ \\
\hline
\end{tabular}

Table S3: Changes in concordance and SCR measures using all 7 recorders, excluding ZA and ZI, or excluding ZB and ZH, based on Oct 6 night data. Sensitivity, specificity, accuracy obtained by comparing manual and automatic annotations over 15 second blocks: presence of call (automatically or manually annotated) in a $15 \mathrm{~s}$ block was considered as positive for that detection method. 


\title{
Epidemic changepoint detection in the presence of nuisance changes
}

\author{
Julius Juodakis ${ }^{1}$, Stephen Marsland
}

Manuscript under review

\begin{abstract}
Many time series problems feature epidemic changes - segments where a parameter deviates from a background baseline. Detection of such changepoints can be improved by accounting for the epidemic structure, but this is currently difficult if the background level is unknown. Furthermore, in practical data the background often undergoes nuisance changes, which interfere with standard estimation techniques and appear as false alarms.

To solve these issues, we develop a new, efficient approach to simultaneously detect epidemic changes and estimate unknown, but fixed, background level, based on a penalised cost. Using it, we build a two-level detector that models and separates nuisance and signal changes. The analytic and computational properties of the proposed methods are established, including consistency and convergence. We demonstrate via simulations that our two-level detector provides accurate estimation of changepoints under a nuisance process, while other state-of-the-art detectors fail. In real-world genomic and demographic datasets, the proposed method identified and localised target events while separating out seasonal variations and experimental artefacts.
\end{abstract}

Keywords Changepoint detection; Piecewise stationary time series; Segmentation; Stochastic gradient methods

\footnotetext{
${ }^{1}$ Corresponding author. Email: julius.juodakis@sms.vuw.ac.nz. J. Juodakis and S. Marsland at School of Mathematics and Statistics, Victoria University of Wellington, New Zealand.
} 


\section{Introduction}

The problem of identifying when the probability distribution of a time series changes - changepoint detection - has been studied since the middle of the 20th century. Early developments stemmed from operations research (Page, 1954). However, as automatic and continuous data collection became more common, many new use cases for changepoint detection have arisen, such as seismic events (Li et al., 2016), epidemic outbreaks (Texier et al., 2016), gravity wave search (McNabb et al., 2004), and network traffic spikes (Hochenbaum et al., 2017). Stimulated by such practical interest, the growth of corresponding statistical theory has been rapid, as reviewed in Aminikhanghahi and Cook (2016), Niu et al. (2016) and Truong et al. (2020).

Different applications pose different statistical challenges. If a single drastic change may be expected, such as when detecting machine failure, the goal is to find a method with minimal time to detection and a controlled false alarm rate (Lau and Tay, 2019). More often, both the number and locations of changepoints must be estimated; the challenge then is to achieve this in a computationally efficient way. Some problems, such as peak detection in sound (Mesaros et al., 2017) or genetic data (Hocking et al., 2017), feature epidemic segments - changepoints followed by a return to the background level - and incorporating this constraint can improve detection or simplify post-processing of ouputs.

Current detection methods that do incorporate a background level assume it to be stable throughout the data (e.g., Fisch et al., 2018; Zhao and Yau, 2019). However, this is not realistic in common applications. In genetic analyses such as measurements of protein binding along DNA there may be large regions where the background level is shifted due to structural variation in the genome or technical artefacts (Zhang et al., 2008). Similarly, a standard task in sound processing is to detect speech in the presence of dynamic background chatter (Mesaros et al., 2017). In various datasets from epidemiology or climatology, such as wave height measurements (Killick et al., 2012), seasonal effects are observed as recurring background changes and will interfere with detection of shorter events. Methods that assume a constant background will be inaccurate in these cases, while ignoring the epidemic structure entirely would cost detection power and complicate the interpretation of outputs.

Our goal is to develop a general method for detecting epidemic changepoints in the presence of nuisance changes in the background. Furthermore, we assume 
that the only available feature for distinguishing the two types of changes is their duration: this would allow analysis of the examples above, which share the property that the nuisance process is slower. The closest research to ours is that of Lau and Tay (2019) for detecting failure of a machine that can be switched on, and thus undergo an irrelevant change in the background level. However, the setting there concerned a single change with known background and nuisance levels; in contrast, we are motivated by the case when multiple changes may be present, with only duration distinguishing their types. Detection then requires two novel developments: 1) rapid estimation of local background level, 2) modelling and distinguishing the two types of potentially overlapping segments.

These developments are presented in this paper as follows: after a background section we present a new algorithm that simultaneously detects epidemic changepoints and estimates the unknown background level (Section 3). The convergence and consistency of this algorithm are proved. While this algorithm is of its own interest, we use it to build a detector that allows local variations in the background, i.e., nuisance changes, in Section 4. In Section 5 we investigate the algorithms with simulations, before showing how the proposed nuisance-robust detector can be applied in two problems: detecting histone modifications in human genome, while ignoring structural variations, and detecting the effects of the COVID-19 pandemic in mortality data, robustly to seasonal effects. Compared to state-of-the-art methods, the proposed detector produced lower false-alarm rates (or more parsimonious models), while retaining accurate detection of true signal peaks.

\section{Background}

In the general changepoint detection setup, the data is a sequence $x_{0: n}=\left\{x_{0}, \ldots, x_{n}\right\}$, split by changepoints $0<\tau_{1}<\tau_{2}<\ldots \tau_{k}<n$ into $k+1$ segments. The observations within each segment are drawn from a distribution $f(x ; \theta)$, with potentially different values of parameter $\theta$ for each segment. The most common example is the change in mean of a Gaussian, i.e., for each $t \in\left[\tau_{i}, \tau_{i+1}\right), x_{t} \sim \mathcal{N}\left(\mu_{i}, \sigma^{2}\right)$, for known, fixed $\sigma^{2}$. (We assume $\theta \in \mathbb{R}^{1}$ to keep notation clearer, but multidimensional problems are also common.)

The aim is to estimate the number and position of all changepoints $\left\{\tau_{i}\right\}$ in the data. A common approach is to use a penalised likelihood cost: define a segment cost function $C\left(x_{a: b} ; \theta\right)=-\log f\left(x_{a: b} ; \theta\right)$, and a penalty $p(k)$ for the number of changepoints $k$. The full cost of $x_{\tau_{0}+1: \tau_{k+1}}$ (where $\tau_{0}=-1$ and $\tau_{k+1}=n$ ) with 
segment parameters $\boldsymbol{\theta}$ then is (here and further vectors are denoted in bold):

$$
F(n ; \tau, \boldsymbol{\theta}, k)=\sum_{i=0}^{k} C\left(x_{\tau_{i}+1: \tau_{i+1}} ; \theta_{i}\right)+p(k) .
$$

Changepoint number and positions are estimated by finding $F(n)=\min F(n ; \tau, \boldsymbol{\theta}, k)$. Such estimation has been shown to be consistent for a range of different data generation models (Fisch et al., 2018; Zheng et al., 2019).

For this problem, computing the true minimum is hard - a naïve brute force approach would require $\mathscr{O}\left(2^{n}\right)$ tests. Approaches to reducing this fall in two broad classes: 1) simplifying the search by memoisation and pruning of paths (Jackson et al., 2005; Killick et al., 2012); 2) using greedy methods to find approximate solutions faster (Fryzlewicz, 2014; Baranowski et al., 2019). In both classes, there are methods that can consistently estimate changepoints in linear time under certain conditions.

The first category is more relevant here. It is based on the Optimal Partitioning (OP) algorithm (Jackson et al., 2005). Let the data be partitioned into discrete blocks $B_{i}: \bigcup_{i} B_{i}=x_{0: n}$, so $B_{i} \cap B_{j}=\emptyset, \forall i \neq j$. A function $V$ that maps each set of blocks $P_{j}=\left\{B_{i}\right\}$ to a cost is block-additive if:

$$
\forall P_{1}, P_{2}, V\left(P_{1} \cup P_{2}\right)=V\left(P_{1}\right)+V\left(P_{2}\right) .
$$

If each segment incurs a fixed penalty $\beta=p(k) / k$, then the cost $F$ defined in (1) is block-additive over segments, and can be defined recursively:

$$
F(s)=\min _{t}\left(F(t)+C\left(x_{t+1: s}\right)+\beta\right) .
$$

In OP, this cost is calculated for each $s \leq n$, and thus its minimisation requires $\mathscr{O}\left(n^{2}\right)$ evaluations of $C$. Furthermore, when the cost function $C$ is such that for all $a \leq b<c$ :

$$
C\left(x_{a: c}\right) \geq C\left(x_{a: b}\right)+C\left(x_{b+1: c}\right)
$$

then at each $s$ it can be determined that some candidate segmentations cannot be "rescued" by further segments, and so they can be pruned from the search space. This approach further reduces the complexity, and gave rise to the family of algorithms called PELT, Pruned Exact Linear Time (Killick et al., 2012). Note that OP and PELT do not rely on any probabilistic assumptions and find the exact minimum. Other pruning schemes are available as well, with different constraints (Maidstone 
et al., 2016).

In this paper, we focus on the epidemic variation of the basic changepoint model - here, a change in regime appears for a finite time and then the process returns to the background level. The pairs of changepoints $s_{i}, e_{i}$ define segments, outside of which the data is drawn from a background distribution $f_{B}$. The data model then becomes:

$$
f\left(x_{t}\right)= \begin{cases}f_{S}\left(x_{t} ; \theta_{i}\right) & \text { if } \exists i: s_{i} \leq t \leq e_{i} \\ f_{B}\left(x_{t}\right) & \text { otherwise. }\end{cases}
$$

Various methods for multiple changepoint detection in this setting have been proposed (Jeng et al., 2010; Fisch et al., 2018; Zhao and Yau, 2019). These use a cost function that includes a separate term $C^{0}$ for the background points:

$$
F\left(n ;\left\{\left(s_{i}, e_{i}\right)\right\}_{k}, \boldsymbol{\theta}, k\right)=\sum_{i=1}^{k} C\left(x_{s_{i}: e_{i}} ; \theta_{i}\right)+C^{0}\left(\left\{x_{t}: t \notin \bigcup_{i}\left[s_{i} ; e_{i}\right]\right\} ; \theta_{0}\right)+p(k)
$$

A common choice for the background distribution is some particular "null" case of the segment distribution family, so that $f_{B}(x)=f_{S}\left(x ; \theta_{0}\right)$ and $C^{0}(\cdot)=-\log f_{B}(\cdot)=$ $C\left(\cdot ; \theta_{0}\right)$. However, while the value of $\theta_{0}$ is known in some settings (such as two copies of DNA in genetic data), often it needs to be estimated. Since $\theta_{0}$ is shared across all background points, the cost function is no longer block-additive as in (2), and OP and PELT algorithms cannot be directly applied.

One solution is to substitute the unknown parameter with some robust estimate of it, based on the unsegmented series $x_{0: n}$. The success of the resulting changepoint estimation then relies on this estimate being sufficiently close to the true value, and so the non-background data fraction must be small (Fisch et al., 2018). This is unlikely to hold in our motivating case, when nuisance changes in the background level are possible.

Another option is to define:

$$
F\left(n ; \theta_{0}\right)=\min _{k,\left\{\left(s_{i}, e_{i}\right)\right\}_{k},\left\{\theta_{i}\right\}_{k}} F\left(n ;\left\{\left(s_{i}, e_{i}\right)\right\}_{k},\left\{\theta_{i}\right\}_{k}, \theta_{0}, k\right),
$$

which can be minimised using OP or PELT, and then minimise it over $\theta_{0}$ using gradient descent (Zhao and Yau, 2019). The main drawback of this approach is an increase in computation time proportional to the number of steps needed for the outer optimisation (typically $>20$ ). 


\section{Detection of changepoints with unknown background level}

To solve the epidemic model in (4) efficiently when the background is unknown and large proportion of non-background data is possible, we introduce Algorithm 1. Similarly to other epidemic changepoint detectors, the algorithm seeks to minimise the penalised likelihood-based cost (5). This is done using OP in steps 3-12, which involves recalculating the optimal segmentation for each new data point.

The main innovation is that we update the estimate of $\theta_{0}$ after each point (step 6): if the new data point is determined to come from the background distribution, the estimate is recalculated from the current background points. For full generality, the algorithm is presented with any estimator $g$ for the background parameter, although we will focus on the case where $g$ is the sample mean or variance. These can be updated using their recursive formulas, which is particularly fast, requiring only $\mathscr{O}(1)$ operations at each point.

The iterative updating produces a precise estimate in a single pass over the data, and so is more computationally efficient than aPELT (Zhao and Yau, 2019), which repeats the entire segmentation with many possible $\theta_{0}$ values. Because the proposed algorithm simultaneously segments and estimates the background, it is also more accurate and robust than methods that attempt to estimate $\theta_{0}$ from unsegmented data, such as CAPA (Fisch et al., 2018).

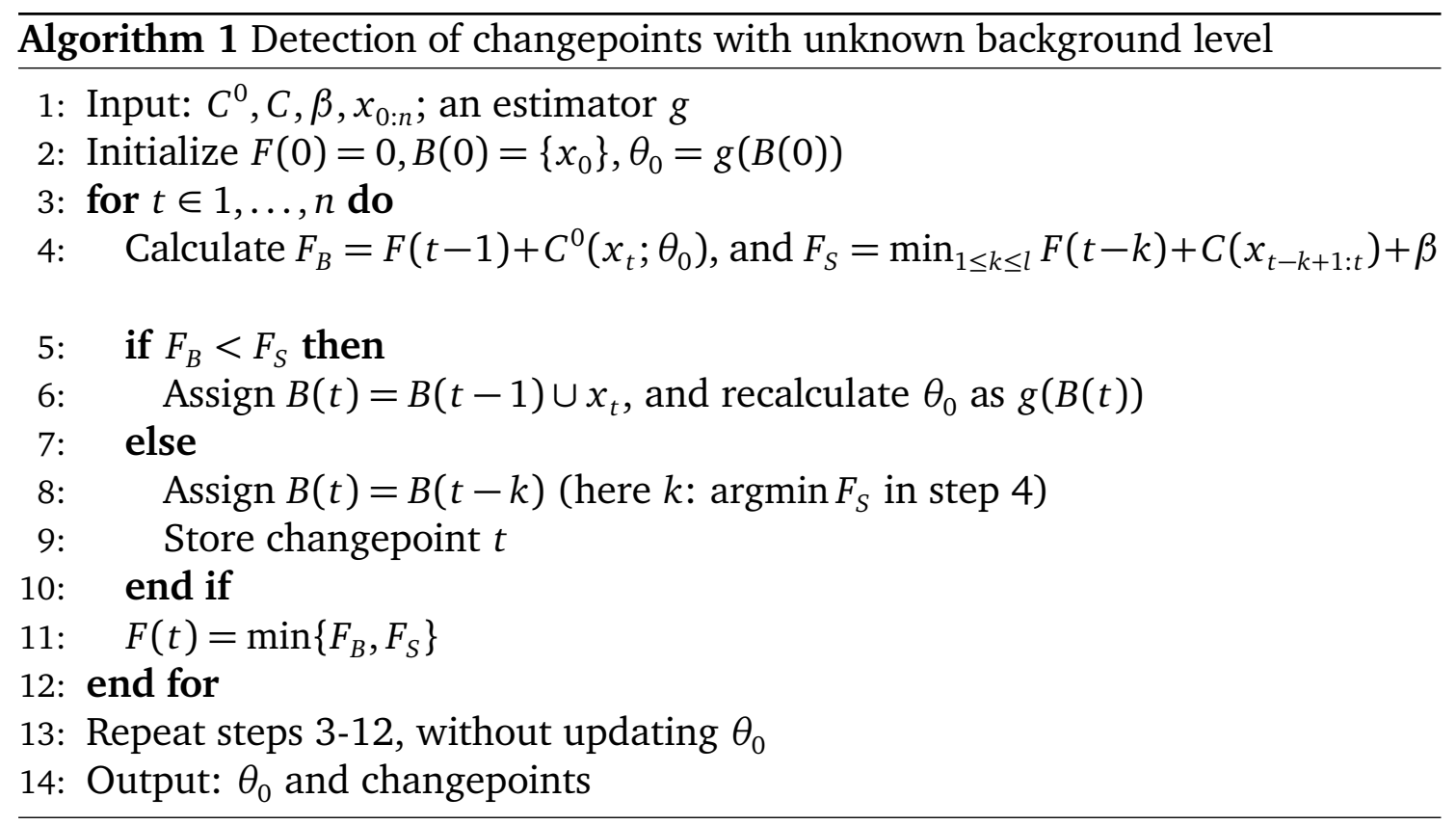


The algorithm as stated here includes a second pass over the data (step 13), repeating the segmentation with the final estimate of $\theta_{0}$. The purpose is to update the changepoint positions that are close to the start of the data and so had been determined using less precise estimates of $\theta_{0}$. This simplifies the theoretical performance analysis, but an attractive option is to use this algorithm in an online manner, without this step. We evaluate the practical consequences of this omission explicitly in Section 5.

The algorithm also includes a search length parameter $l$ : this may be set to limit segment duration (as will be used later in the paper for signal segments), or kept unlimited, as $l=n$.

We demonstrate some theoretical properties of Algorithm 1 next.

\subsection{Convergence}

The changepoint model can be understood as a function over an interval that is sampled to obtain $n$ observed points. We explore the properties of the algorithm as the sampling density increases: in this setting, the number and strength of changes are fixed, but the length of segments grows as $o(n)$.

Theorem 1. Consider the problem of an epidemic change in mean, with data $x_{0: n}$ generated as in (4). Assume the $p d f f_{B}(x)$ and marginal pdf of segment points $f_{S}(x)$ are symmetric and strongly unimodal, with unknown background mean $\theta_{0}$, and that data points within each segment are iid. Denote by $w_{t}$ the estimate of $\theta_{0}$ obtained by analysing $x_{0: t}$ by Algorithm 1. The sequence $\left\{w_{t}\right\}$ converges:

1. to the true background value $\theta_{0}$ almost surely, if $\int_{-\infty}^{\infty} x f_{S}(x) d x=\theta_{0}$.

2. to a neighbourhood $\left(\theta_{0}-\epsilon, \theta_{0}+\epsilon\right)$ almost surely, where $\epsilon \rightarrow 0$ as the number of background points $n$ between successive segments $n \rightarrow \infty$.

We refer the reader to Supplementary Material A for the proof. It is based on a result by Bottou (1998), who established conditions in which an online minimisation algorithm almost surely converges to the optimum. We show that in the first case, the updating process in our algorithm satisfies these conditions directly. (This case could arise with various combinations of fixed $\theta_{i}$, or, for example, if the segment means are modelled as coming from a Gaussian prior centred around $\theta_{0}$.) In the second case, weak changes may be missed and cause $w_{t}$ to deviate from $\theta_{0}$. Then the conditions can be satisfied by defining update cycles comprising points between successive misclassifications. As $n$ increases, weaker changes get detected accurately, so the frequency of misclassification drops and convergence improves. 


\subsection{Consistency for Gaussian data}

As the sampling density increases, more accurate estimation of the number and locations of changepoints is expected; this property is formalised as consistency of the detector. Fisch et al. (2018) showed that detectors based on minimising penalised cost are consistent for Gaussian data, and their result can be adapted to prove consistency of Algorithm 1. The strengthened SIC penalty $\alpha \log (n)^{1+\delta}$ is used. Additionally, following Fisch et al. (2018), we set the following minimum signal strength bound:

$$
\forall i,\left(e_{i}-s_{i}\right) \Delta_{i}>\log (n)^{1+\delta},
$$

where $\Delta_{i}$ represents the strength of change associated with segment $i$, relative to the background parameters $\mu_{0}, \sigma_{0}$ :

$$
\Delta_{i}=\min \left(\tilde{\Delta}_{i}, \tilde{\Delta}_{i}^{2}\right), \text { with } \tilde{\Delta}_{i}=\log \left(\frac{\left(\mu_{0}-\mu_{i}\right)^{2}+2\left(\sigma^{2}+\sigma_{i}^{2}\right)}{4 \sigma_{0} \sigma_{i}}\right)
$$

Theorem 2. Let the data $x_{0: n}$ be generated from the epidemic changepoint model (4), with $f_{B}$ and $f_{S}$ Gaussian, and the changing parameter $\theta$ is either its mean or variance (assume the other parameter is known). Further, assume (6) holds for $k$ changepoints. Analyse the data by Algorithm 1 with penalty $\beta=\alpha \log (n)^{1+\delta}, \alpha, \delta>0$. The estimated number and position of changepoints will be consistent, i.e. $\forall \epsilon>0, n>B$ :

$$
P\left(\hat{k}=k, \max \left\{\left|\hat{s}_{i}-s_{i}\right|,\left|\hat{e}_{i}-e_{i}\right|\right\}<\frac{A}{\Delta_{i}} \log (n)^{1+\delta}, \forall 1 \leq i \leq k\right) \geq 1-C n^{-\epsilon},
$$

for some A, B, C that do not increase with $n$.

The proof is given in Supplementary Material B. We use the connection between Algorithm 1 and stochastic gradient descent to establish error bounds on the background parameter estimates. These bounds then allow us to apply a previous consistency result (Fisch et al., 2018) to our case.

Remark. Theorem 2 still holds if the other parameter is not known, but estimated to a precision of $\mathscr{O}(\sqrt{\log (n) / n})$. As shown in Fisch et al. (2018), if the nonbackground contamination is limited, this is satisfied by robust estimators such as the interquartile range for scale.

Standard PELT-style pruning can be applied to Algorithm 1, with most likelihoodbased costs. We detail the corresponding implementation and show that it does not change the optimisation result in Supplementary Material C. 


\section{Detecting changepoints with a nuisance process}

\subsection{Problem setup}

In this section, we consider the changepoint detection problem when there is an interfering nuisance process. We assume that this process, like the signal, consists of segments, which we denote by $s_{j}^{N}, e_{j}^{N}$. Data within these segments is generated from a nuisance-only distribution $f_{N}$, or from some distribution $f_{N S}$ if a signal occurs at the same time. In total, four states are possible, so the overall model of data is:

$$
f\left(x_{t}\right)= \begin{cases}f_{N S}\left(x_{t} ; \theta_{i}, \theta_{j}^{N}\right) & \text { if } \exists i, j: t \in\left[s_{i} ; e_{i}\right] \cap\left[s_{j}^{N}, e_{j}^{N}\right] \\ f_{S}\left(x_{t} ; \theta_{i}\right) & \text { if } \exists i: t \in\left[s_{i}, e_{i}\right], t \notin \cup_{j}\left[s_{j}^{N}, e_{j}^{N}\right] \\ f_{N}\left(x_{t} ; \theta_{j}^{N}\right) & \text { if } \exists j: t \in\left[s_{j}^{N}, e_{j}^{N}\right], t \notin \cup_{i}\left[s_{i}, e_{i}\right] \\ f_{B}\left(x_{t}\right) & \text { otherwise }\end{cases}
$$

We add two more conditions to ensure identifiability:

1. The nuisance process evolves more slowly than the signal process, so $\min \left(e_{j}^{N}-\right.$ $\left.s_{j}^{N}\right)>\max \left(e_{i}-s_{i}\right)$.

2. Signal segments are either entirely contained within a nuisance segment, or entirely out of it:

$$
\forall i, j \text {, either }\left[s_{i}, e_{i}\right] \subset\left(s_{j}^{N}, e_{j}^{N}\right) \text {, or }\left[s_{i}, e_{i}\right] \cap\left[s_{j}^{N}, e_{j}^{N}\right]=\emptyset
$$

Remark. In practice, there should be a sufficient gap between these lengths to allow for error in $s, e$ estimates, typically of order $o(\log n)$. This is satisfied in our setting where the changepoint positions are fixed. Violating the second condition produces several shorter segments which cannot be unambiguously resolved, but in practice will result in one or more detections near the true signal segment and thus is not a major obstacle.

To define the penalised cost of such a model, let $X_{S}=\bigcup_{i} x_{s_{i}: e_{i}}, X_{N}=\bigcup_{j} x_{s_{j}^{N}: e_{j}^{N}}$, set penalties $p(k)=\beta k, p^{\prime}(m)=\beta^{\prime} m$ for the numbers of signal and nuisance segments, respectively, and cost functions $C^{N S}, C^{S}, C^{N}, C^{0}$ corresponding to the log- 
likelihoods of each of the distributions in (8). Then the full cost is:

$$
\begin{aligned}
& F\left(n ;\left\{\left(s_{i}, e_{i}\right)\right\}_{k}, k,\left\{\left(s_{j}^{N}, e_{j}^{N}\right)\right\}_{m}, m, \boldsymbol{\theta}\right)=C^{0}\left(x_{0: n} \backslash\left(X_{S} \cup X_{N}\right)\right)+\sum_{i=0}^{k} C^{S}\left(x_{s_{i}: e_{i}} \backslash X_{N} ; \theta_{i}\right)+ \\
& \quad+\sum_{j=0}^{m}\left(C^{N}\left(x_{s_{j}^{N}: e_{j}^{N}} \backslash X_{S} ; \theta_{j}^{N}\right)+\sum_{i=0}^{k} C^{N S}\left(x_{s_{j}^{N}: e_{j}^{N}} \cap x_{s_{i}: e_{i}} ; \theta_{i}, \theta_{j}^{N}\right)\right)+\beta k+\beta^{\prime} m .
\end{aligned}
$$

\subsection{Proposed method}

To minimise the cost in (10), first notice that it can also be expressed, using $k_{j}$ as the number of signal segments that overlap a nuisance segment $j$ and $k_{0}=k-\sum k_{j}$, as:

$$
\begin{aligned}
F\left(n ;\left\{\left(s_{i}, e_{i}\right)\right\}_{k}, k,\left\{\left(s_{j}^{N}, e_{j}^{N}\right)\right\}_{m},\right. & m, \boldsymbol{\theta})=C^{0}\left(x_{0: n} \backslash\left(X_{S} \cup X_{N}\right)\right)+ \\
& +\sum_{i=0}^{k_{0}} C^{S}\left(x_{s_{i}: e_{i}} \backslash X_{N} ; \theta_{i}\right)+\beta k_{0}+\sum_{j=0}^{m}\left(C^{\prime}\left(x_{s_{j}^{N}: e_{j}^{N}}\right)+\beta^{\prime}\right) .
\end{aligned}
$$

with $C^{\prime}(\cdot)=F\left(\cdot ;\left\{\left(s_{i}, e_{i}\right)\right\}_{k_{j}},\{\theta\}_{k_{j}}, k_{j}\right)$, the standard epidemic cost in (5). That is, over each proposed nuisance segment $s_{j}^{N}: e_{j}^{N}$, an epidemic changepoint problem with unknown background parameter must be solved. Condition (9) ensures that $C^{\prime}, C^{S}, C^{0}$ are independent (do not share any points or parameters), so $F$ is blockadditive and can be minimised by $\mathrm{OP}$.

A method to minimise this cost is outlined in Algorithm 2. In it, an outer loop proceeds over the data to identify segments by the usual OP approach. Length bound $l$ determines which type of cost is applied: signal segment $\operatorname{cost} C^{S}$, or nuisance (with potential signals) $C^{\prime}$. If $C^{\prime}$ is needed, it is minimised in an inner loop using Algorithm 1. This is a key difference from related methods: evaluating more complex costs $C^{\prime}$ is only possible because of the efficiency of Algorithm 1.

By Theorem 2, this process will estimate the number and positions of true segments consistently given accurate assignment of $s_{j}^{N}, e_{j}^{N}$. However, the latter event is subject to a complex set of assumptions on relative signal strength, position, and duration of the segments. Therefore, we do not attempt to describe these in full here, but instead investigate the performance of the method by extensive simulations in Section 5.3.

Algorithm 2 is stated assuming that a known or estimated value of the parameter $\theta_{0}$, corresponding to the background level without the nuisance variations, is available. In practice, it may be known when there is a technical noise floor or 
a meaningful baseline that can be expected after removing the nuisance changes. Alternatively, $\theta_{0}$ may be substituted by a robust estimate, or the method can be modified to estimate it simultaneously with segmentation, using a principle similar to Algorithm 1.

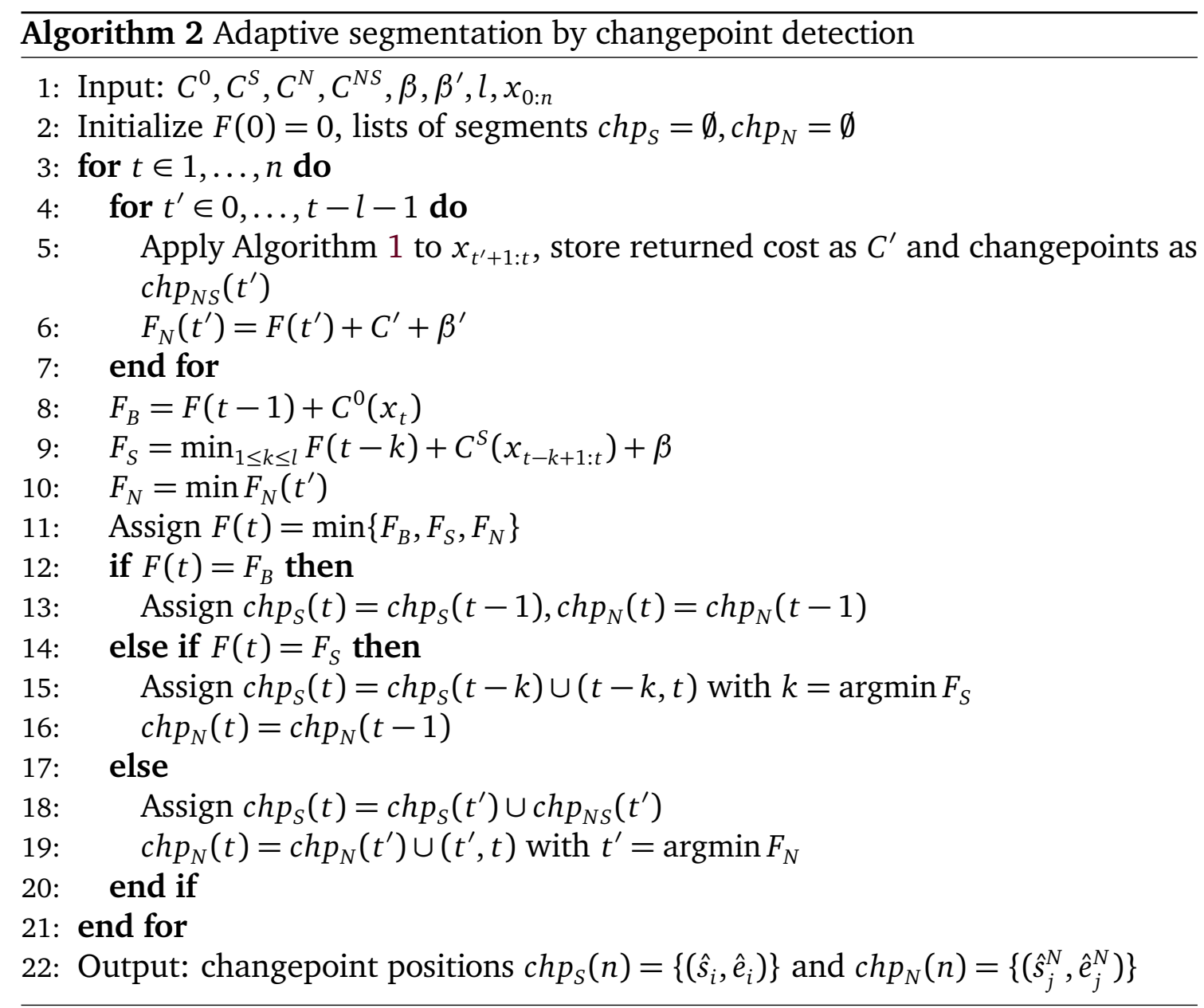

\subsection{Pruning}

In the proposed method, the estimation of the mean of segment $j$ is sensitive to the segment length, therefore the cost $C^{\prime}$ is not necessarily block-additive (3), and so it cannot be guaranteed that PELT-like pruning will be exact. However, we can establish a local pruning scheme that retains the exact optimum with probability $\rightarrow 1$ as $n \rightarrow \infty$.

Proposition 1. Assume data $x_{0: n}$ is generated from a Gaussian epidemic changepoint model, and that the distance between changepoints is bounded by some function $A(n)$ :

$$
\forall i, j, j^{\prime}: \min \left\{\left|s_{j}^{N}-e_{j^{\prime}}^{N}\right|,\left|s_{i}-s_{j}^{N}\right|,\left|e_{i}-e_{j}^{N}\right|\right\}>A(n)
$$


At time $t$, the solution space is pruned by removing:

$$
\boldsymbol{k}_{p r, t}=\left\{k: F(k-1)+C^{\prime}\left(x_{k: t}\right) \geq \min _{m} F(m-1)+C^{\prime}\left(x_{m: t}\right)+\alpha \log (n)^{1+\delta}\right\} .
$$

Here $m \in(t-A(n) ; t], k \in(t-A(n) ; t], k \neq m$. Then $\forall \epsilon>0$, there exist constants $B, n_{0}$, such that when $n>n_{0}$, the true nuisance segment positions are retained with high probability:

$$
P\left(\forall j: s_{j}^{N} \notin \bigcup_{t} \boldsymbol{k}_{p r, t}, e_{j}^{N} \notin \bigcup_{t} \boldsymbol{k}_{p r, t}\right) \geq 1-B n^{-\epsilon} .
$$

The proof is given in Supplementary Material D. The assumed distance bound serves to simplify the detection problem: within each window $(t-A(n), t]$, at most 1 true changepoint may be present, and the initial part of Algorithm 2 is identical to a standard epidemic changepoint detector. It can be shown that other candidate segmentations in the pruning window are unlikely to have significantly lower cost than the one associated with $s_{j}^{N}, e_{j}^{N}$, and therefore $s_{j}^{N}, e_{j}^{N}$ are likely to be retained in pruning.

This scheme only prunes within windows of user-set size $A(n)$ and so is less efficient. By choosing large $A(n)$, the efficiency can be increased, but that may violate the data generation model and cause some true changepoints to be lost. However, assuming that the overall estimation of nuisance changepoints is consistent, Proposition 1 extends to standard pruning over the full dataset. We show that this holds empirically in Section 5.3. The overall computational complexity of this algorithm then is similar to PELT, $\mathscr{O}(n)$ in the best case.

\section{Simulations}

In this section, we present the results of simulations used to evaluate the performance of the methods. Further details of simulation settings are presented in Supplementary Material E. The corresponding $\mathrm{R}$ code is available at https: //github.com/jjuod/changepoint-detection.

\subsection{Algorithm 1 estimates the background level consistently}

Firstly, we tested that Algorithm 1 estimates the background parameter accurately (Theorem 1). Three different scenarios with changes in mean were investigated: Gaussian data with one segment (one segment), multiple segments (multiple), or 
non-Gaussian data (heavy tail). Background $\theta_{0}$ was estimated using Algorithm 1 , or by median of all points (as in Fisch et al. (2018)), or by an oracle estimator. The proposed algorithm was applied with the Gaussian cost throughout this section. No nuisance segments were present.

As seen in Figure 1, Algorithm 1 produced consistent and efficient estimates of the background level. Estimation by the median of the non-segmented data was biased (since it includes segment points), although it may be preferrable at low $n$, where our estimate showed large variance. At $n>400$, our algorithm provides the lowest total error in all tested scenarios, even with the mis-specified cost function in the heavy tail scenario.

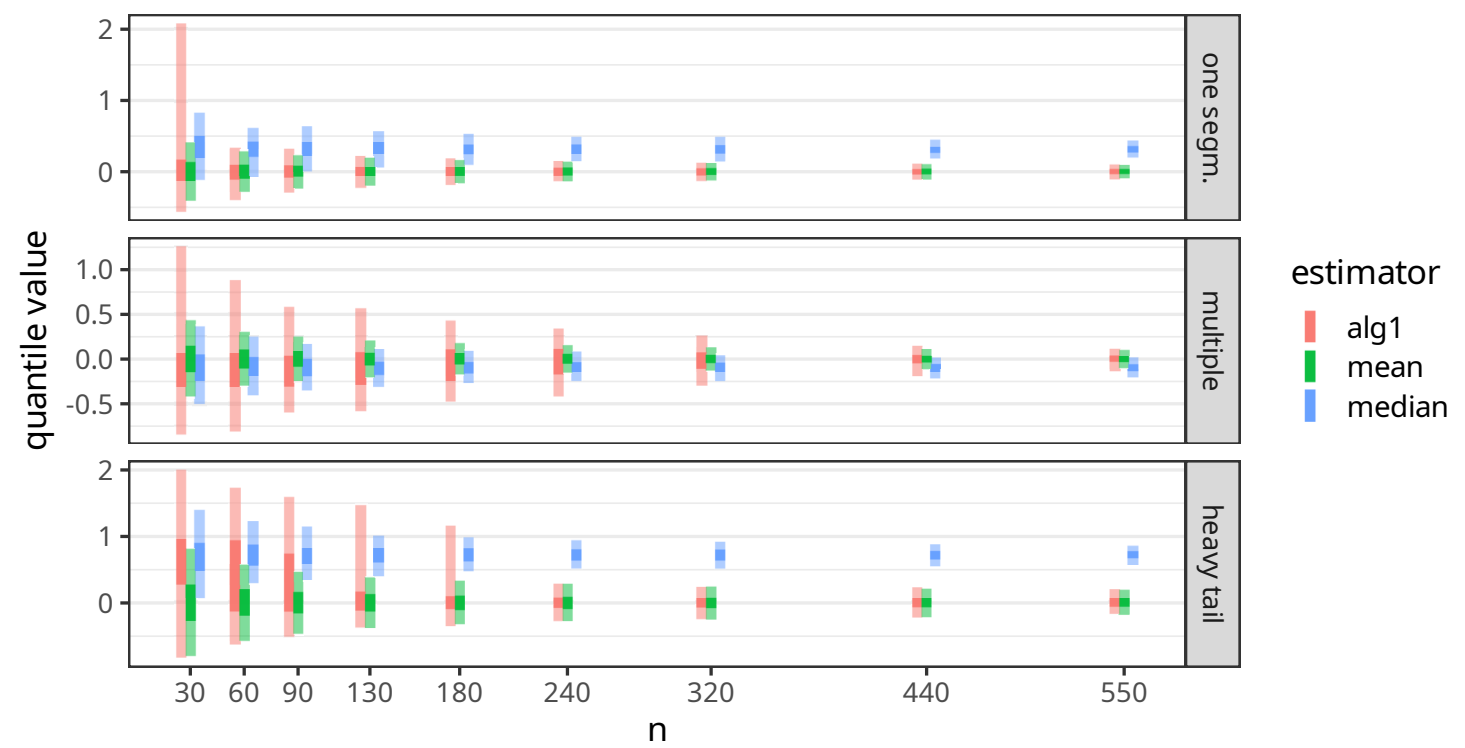

Figure 1: Consistency of the background level estimation. Time series simulated in three different scenarios were analysed by Algorithm 1 (shown in red). Lines are the interquartile range (solid) and 2.5-97.5\% range (faint) of the background parameter estimates observed in 500 replications. For comparison, we show ranges of an oracle estimator (mean of true background points; green) and median of all points (blue).

\subsection{Segment positions are accurately estimated by Algorithm 1}

The same setup was used to evaluate the estimation of segment number and positions. In all three scenarios, the true positive rate (fraction of simulations in which all changes were detected) approaches 1 (Table 1 ). When the signal is strong (one segment scenario), the segmentation was accurate even at $n=30$. In scenario heavy tail, the algorithm correctly detected changes at the true segment start and end, but tended to fit the segment as multiple ones, due to the heavy tails of the $t$ 
distribution.

Table 1: Consistency of the estimated changepoints. Time series simulated in three different scenarios were analysed using Algorithm 1, in 500 replications for each $n$. The mean number of reported segments and the TPR (fraction of replications when a changepoint was detected within $0.05 n$ of each true changepoint) are shown. The number of true segments was 1,3 , and 1 for the one, multiple and heavy scenarios, respectively.

\begin{tabular}{lrrrrrr}
\hline & \multicolumn{2}{c}{ one segment } & \multicolumn{2}{c}{ multiple } & \multicolumn{2}{c}{ heavy tail } \\
$n$ & Mean \# seg. & TPR & Mean \# seg. & TPR & Mean \# seg. & TPR \\
\hline 30 & 1.112 & 0.932 & 0.552 & 0.000 & 0.644 & 0.142 \\
90 & 1.040 & 0.998 & 1.096 & 0.008 & 1.426 & 0.592 \\
180 & 1.054 & 1.000 & 1.762 & 0.086 & 1.930 & 0.878 \\
440 & 1.032 & 1.000 & 2.900 & 0.824 & 2.798 & 0.998 \\
750 & 1.028 & 1.000 & 3.010 & 0.992 & 3.692 & 1.000 \\
\hline
\end{tabular}

We also retrieved the changepoint positions that were estimated in step 12 . This corresponds to the online usage of the algorithm, in which segmentation is not repeated after the first pass over the data. This had very little impact on the result accuracy (Table S3 in Supplementary Material F), suggesting that this simplification can be safely used.

\subsection{Algorithm 2 recovers true segments under interference}

Algorithm 2 was also evaluated in three different scenarios of Gaussian data with changes in mean. Scenarios 1 and 2 had both nuisance and signal segments, while scenario 3 contained many weak signal segments. The series were analysed by Algorithm 2 (proposed), three alternative epidemic detectors, and the narrowestover-threshold detector not. The latter is a state-of-the-art non-epidemic detector, and so does not incorporate a shared background structure (Baranowski et al., 2019).

We also compared Algorithm 2 when applied without pruning, or with global pruning as in (11), with $m \in(0 ; t-l)$ at each $t$. In scenarios 1 and 2 , pruning changed the result in only 3 out of 5000 runs (Table S5 in Supplementary Material F), although some more differences were observed when many weak changes were present. As the results were mostly identical, we only present results obtained with pruning from here on.

We observed that the proposed method (with pruning) successfully detected true signal segments in scenarios 1 and 2 (Figure 2 and Table 2). The number of nuisance detections was accurate in scenario 1 , and slightly underestimated in 
favour of more signal segments in scenario 2, most likely because the set nuisance length was close to the cutoff $l$.
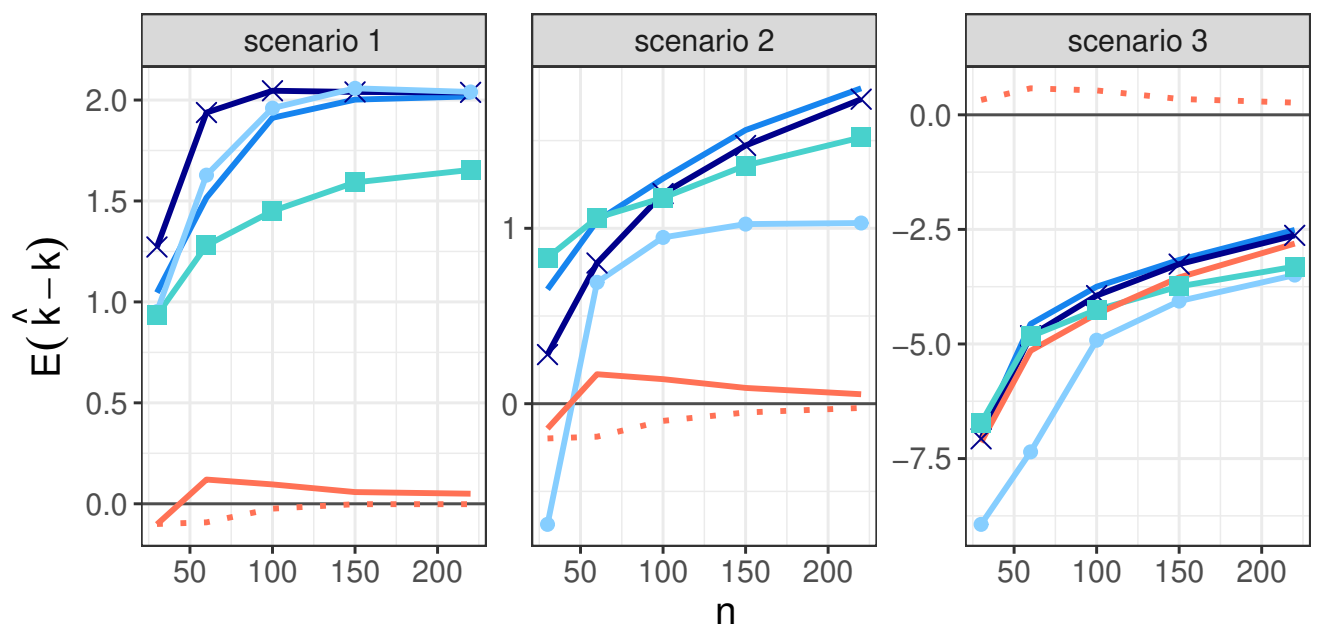

$$
\begin{aligned}
\text { detections: } & - \text { anomaly }=\text { sparse }- \text { proposed } \\
* \text { aPELT } & - \text { not }
\end{aligned}
$$

Figure 2: Relative bias in the number of changepoints estimated by the proposed Algorithm 2 (pruned), and four alternative detectors. Data simulated in 500 replications. For the proposed algorithm, bias is calculated separately in signal (solid line) and nuisance (dashed) segments.

As expected, the reference methods which do not include nuisance segments in the model identify them as multiple changepoints; as a result, the number of segments was over-estimated up to 3-fold. These models are also unable to capture the signal-specific change in mean $\theta^{S}$ : anomaly estimated $\hat{\theta}^{S}=4.00$, and not estimated $\hat{\theta}^{S}=3.96$ in scenario 1 at $n=220$. These values correspond to the sum of the signal and nuisance effects. While the estimation is accurate and could be used to recover the signal-specific change by post-hoc analysis, our proposed method estimated it directly, as $\hat{\theta}^{S}=1.99$ in scenario 1 at $n=220$.

Scenario 3 presented signal changes that were weak $(<\sigma$ in $25 \%$ of the segments) and short (1-point long at $n=30$ ). This imposed a fundamental limit on detection by penalised cost: all epidemic detectors missed a similar number of segments, as seen in Figure 2. However, not performed consistently worse, showing the benefits of utilising the background structure, especially at smaller sampling densities. While there were no nuisance segments here, the ability to estimate them did not harm the proposed method, and it was on par with the best detectors. For all methods, larger $n$ would be needed to detect the weakest segments in this setup. 
Table 2: The true positive rate of changepoint estimation by the proposed Algorithm 2 and alternative detectors. Data simulated in 500 replications. The true positive rate is the fraction of iterations when a (signal) changepoint was detected within $0.05 n$ of each true signal changepoint.

\begin{tabular}{ccccccc}
\hline Scenario & $n$ & Proposed & anomaly & aPELT & sparse & not \\
\hline 1 & 30 & 0.460 & 0.436 & 0.616 & 0.180 & 0.464 \\
& 60 & 0.732 & 0.536 & 0.820 & 0.072 & 0.706 \\
& 150 & 0.994 & 0.992 & 0.992 & 0.054 & 0.988 \\
& 220 & 0.998 & 1.000 & 0.998 & 0.018 & 1.000 \\
\hline 2 & 30 & 0.644 & 0.930 & 0.858 & 0.964 & 0.128 \\
& 60 & 0.894 & 0.984 & 0.974 & 0.982 & 0.824 \\
& 150 & 0.998 & 1.000 & 1.000 & 1.000 & 0.998 \\
& 220 & 0.994 & 1.000 & 1.000 & 1.000 & 1.000 \\
\hline 3 & 30 & 0.000 & 0.000 & 0.000 & 0.000 & 0.000 \\
& 60 & 0.002 & 0.008 & 0.002 & 0.006 & 0.000 \\
& 150 & 0.022 & 0.028 & 0.014 & 0.024 & 0.006 \\
& 220 & 0.058 & 0.068 & 0.054 & 0.034 & 0.018 \\
\hline
\end{tabular}

\section{Real-world data}

In this section, we present the results of real data analysis using the proposed method. Data sources and additional experiment details are presented in Supplementary Material E. The corresponding R code is available at https://github. com/jjuod/changepoint-detection.

\subsection{ChIP-seq}

As an example application of the algorithms proposed in this paper, we demonstrate peak detection in chromatin immunoprecipitation sequencing (ChIP-seq) data. The goal of ChIP-seq is to identify DNA locations where a particular protein of interest binds, by precipitating and sequencing bound DNA. This produces a density of binding events along the genome that then needs to be processed to identify discrete peaks. Typically, some knowledge of the expected peak length is available to the researcher. Furthermore, the background level may contain local shifts of various sizes, caused by sequencing bias or true structural variation in the genome (Zhang et al., 2008). The method proposed in this paper is designed for such cases, and can potentially provide more accurate and more robust detection.

We used two binding density series obtained in ChIP-seq experiments (see Sup- 
plementary Material E for details). Broad Institute H3K27ac data is not annotated, but includes a control track which reveals the presence of some nuisance variation (Figure 3, top). UCI/McGill dataset was previously used for evaluating peak detectors (Hocking et al., 2017, 2018). It includes annotations based on visual inspection. These are weak in the sense that they indicate peak presence or absence in a region, not their exact positions, to acknowledge the labelling subjectivity. The series were analysed by the proposed Algorithm 2 and three alternative changepoint detectors, including PeakSegDisk (Hocking et al., 2018), developed specifically for ChIP-seq data processing. Note that the proposed algorithm was used with a cost based on Gaussian likelihood, as presented so far, even though the data are positive counts.

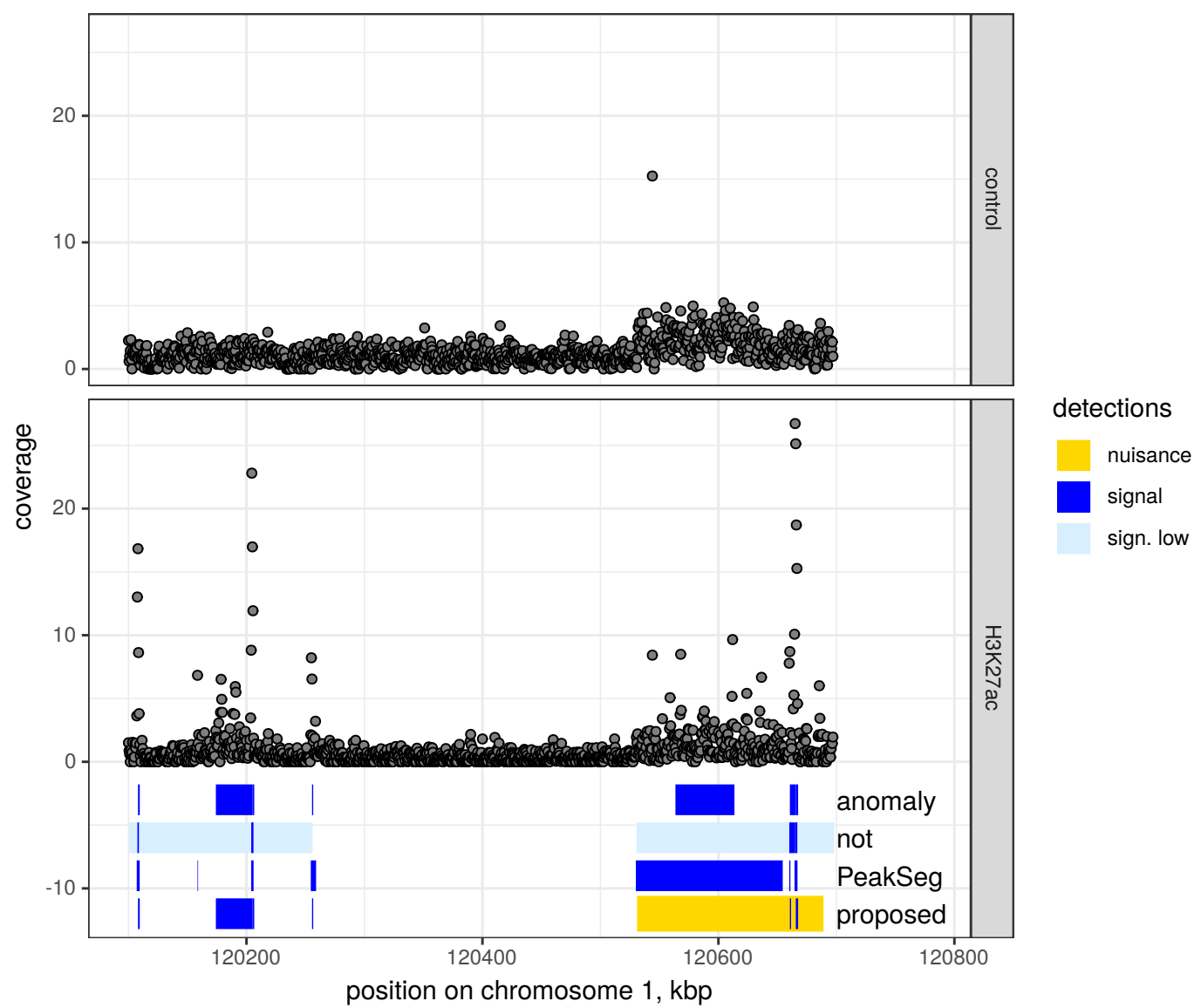

Figure 3: ChIP-seq read counts and analysis results. Counts provided as mean coverage in $500 \mathrm{bp}$ windows for a non-specific control sample (top) and H3K27ac histone modification (bottom), chromosome 1. Segments detected in the H3K27ac data by the method proposed here (Algorithm 2) and three other detectors are shown under the counts. Note that the proposed method can also produce longer nuisance changes overlapped by signal segments. not does not specifically identify background segments; we show the ones with relatively low mean in light colour. 
In the H3K27ac data, all methods detected the three most prominent peaks, but produced different results for smaller peaks and more diffuse change areas (Figure 3, bottom). Both PeakSegDisk and anomaly marked a broad segment in the area around 120,600,000 bp. Based on comparison with the control data, this change is spurious, and it exceeds the $50 \mathrm{kbp}$ bound set for target segments. While this bound was provided to the anomaly detector, it does not include an alternative way to model these changes, and therefore still reports one or more shorter segments. In contrast, our method accurately modelled the area as a nuisance segment with two overlapping sharp peaks, even with data clearly deviating from the assumed Gaussian model.

Using not, the data was partitioned into 10 segments. By defining segments with low mean $\left(\theta<\hat{\mu}_{0}+\hat{\sigma}_{0}\right)$ as background, we could reduce this to 4 signal segments; while this removed the spurious background change, it also discarded the shorter change around 120,200,000 bp, which fits the definition of a signal peak ( $<50 \mathrm{kbp}$ ) and was retained by the proposed method. This data illustrates that choosing the post-processing required for most approaches is not trivial, and can have a large impact on the results. In contrast, the parameters required for our method have a natural interpretation and may be known a priori or easily estimated, and the outputs are provided in a directly useful form.

In the UCI data, segment detections also generally matched the visually determined labels (Figure 4). However, our method produced the most parsimonious models to explain the changes, reporting two nuisance segments and a single sharp peak around $62,750,000 \mathrm{bp}$. The nuisance segments correspond to broad regions of mean shift, which were also detected by anomaly and not, but using 6 and 16 segments, respectively. Notably, PeakSeg differed considerably: as this method does not incorporate a single background level, but requires segments to alternate between background and signal, the area around 62,750,000 bp was defined as background, despite having a mean of $4.5 \hat{\mu}_{0}$. In total, 12 segments were reported by this method. This shows that the ability to separate nuisance and signal segments helps produce more parsimonious models, and in this way minimises the downstream efforts such as experimental replication of the peaks.

The visual annotations provided for this region are shown in the first row in Figure 4. Note that they do not distinguish between narrow and broad peaks (single annotations in this sample range up to $690 \mathrm{~kb}$ in size). Furthermore, comparison with such labels does not account for finer segmentation, coverage in the peak area, or the number of false alarms outside it. For these reasons we are unable to use the 
labels in a quantitative way.

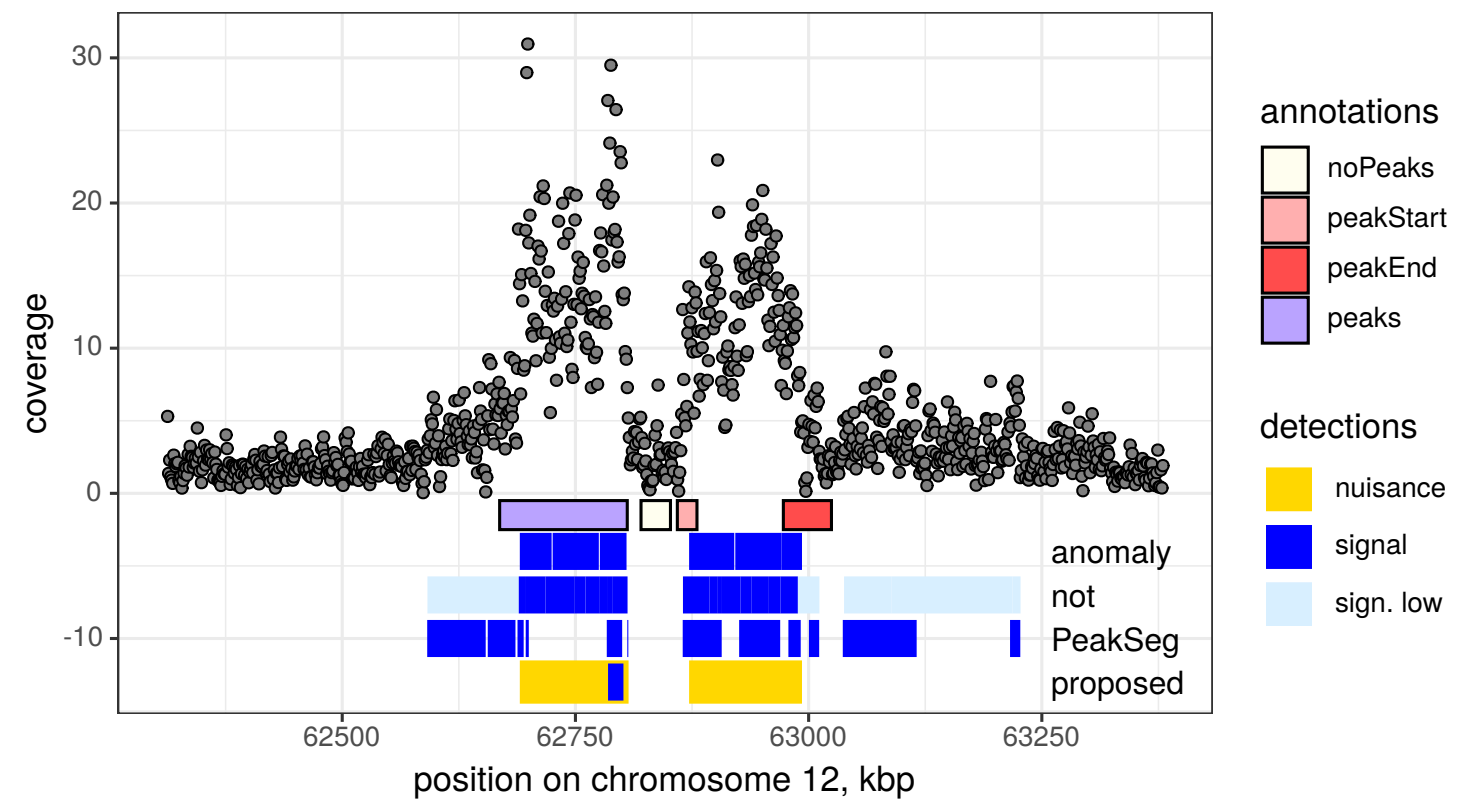

Figure 4: UCI/McGill ChIP-seq data: read coverage in 1100 bp windows, black points, and manual annotations of peaks (boxes). Detection results using Algorithm 2 proposed in this paper, as well as three state-of-the-art methods are shown at the bottom.

For a quantitative comparison of the detectors, we use SIC. The proposed method is favoured in both datasets, producing SIC of 4285.8 in the Broad data, while PeakSeg, not and anomaly had an SIC of 4447.4, 4532.2, and 4311.6, respectively. The smallest SIC values in UCI data were also produced by our method (4664.5) and by anomaly (4664.8), while not resulted in an SIC of 4705.7 and PeakSeg 6886.7. This indicates that in addition to the practical benefits, the nuisance-signal structure provides a better fit to these series than models that allow only one type of segments.

\subsection{European mortality data}

The recent pandemic of coronavirus disease COVID-19 prompted a renewed interest in early outbreak detection and quantification. In particular, analysis of mortality data provided an important resource for guiding the public health responses to it (e.g. Baud et al., 2020; Yuan et al., 2020).

We analysed weekly mortality in 60-64 year age group in Spain over a three year period, using the four methods introduced earlier. Besides the impact of the pandemic, these data contain longer nuisance increases corresponding to winter seasons. As before, Gaussian likelihood was assumed for the proposed Algorithm 2. 


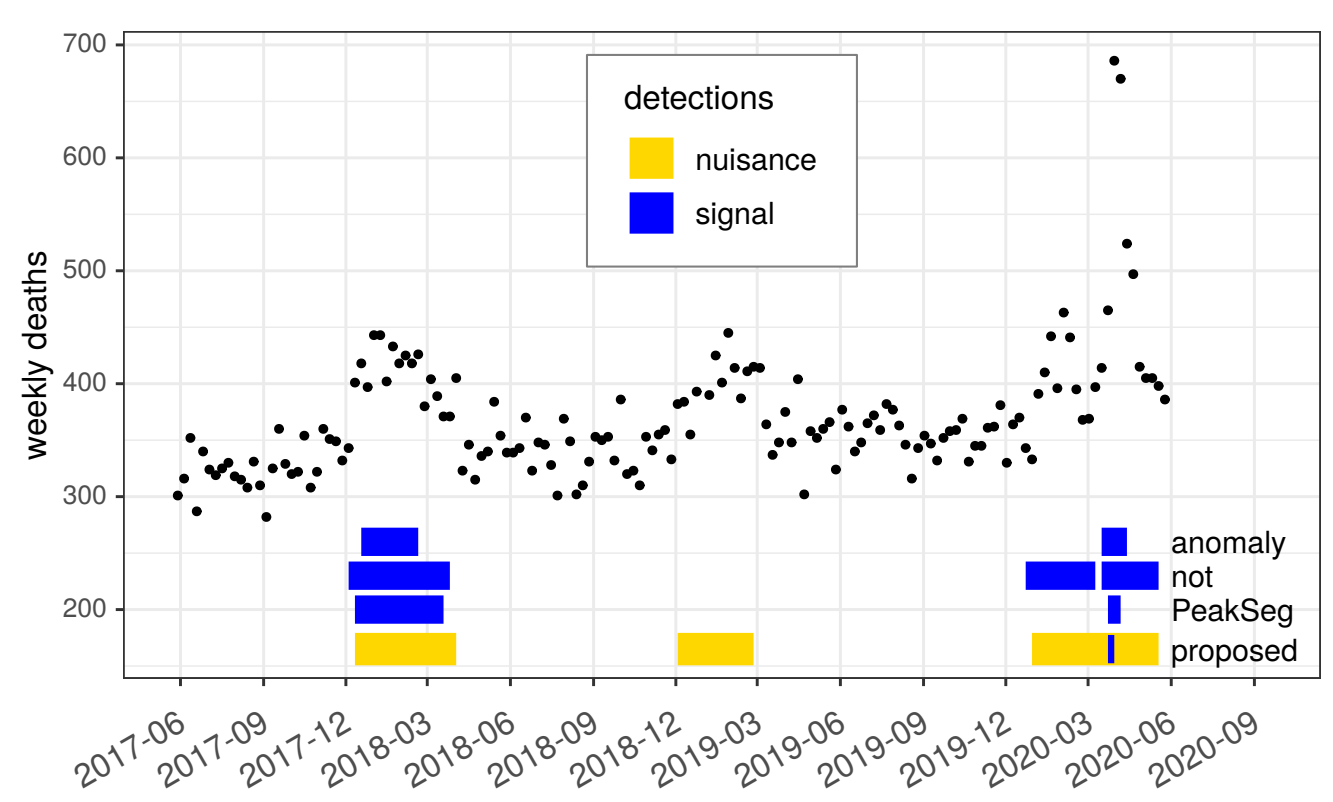

Figure 5: Weekly deaths in Spain, in the 60-64 years age group, over 2017-2020 (black points). Detection results using the method proposed in this paper and three alternative methods shown as lines below.

The detected segments are shown in Figure 5. Three of the methods, anomaly, PeakSeg, and Algorithm 2, detected a sharp peak around the pandemic period. However, anomaly and PeakSeg also marked one winter period as a signal segment, while ignoring the other two. Four segments were created by not, including a broad peak continuing well past the end of the pandemic spike. In contrast, the proposed method marked the pandemic spike sharply, while also labelling all winter periods as nuisance segments. The resulting detection is again parsimonious and flexible: if only short peaks are of interest, our method reports those with lower false alarm rate than the other methods, but broader segments are also marked accurately and can be retrieved if relevant.

As in the ChIP-seq data, comparing the results by SIC identifies our method as optimal for this dataset (SIC of 1568.2 for Algorithm 2 vs. 1629.3, 1648.2, and 1626.3 for the other methods). Note that the SIC penalizes both signal and nuisance segments, so in this case our model still appears optimal despite having more parameters.

\section{Discussion}

In this paper, we have presented a pair of algorithms for improving detection of epidemic changepoints. Similarly to stochastic gradient descent, the iterative updating 
of the background estimate in Algorithm 1 leads to fast convergence while allowing large fraction of non-background points. This is utilised in Algorithm 2 to analyse nuisance-signal overlaps. We have shown in the simulations that the algorithms outperform state-of-the-art methods, producing better background estimates and fewer false positives. While the simulations and theoretical analysis focus on the specific model of Gaussian data and step-like changes, practical results show that the detection is robust and can be usefully applied to data that strongly deviates from these assumptions.

The computational complexity of both algorithms presented here is $\mathscr{O}(n)$ in the best case, which is similar to state-of-the-art methods (Killick et al., 2012; Hocking et al., 2018). However, this is stated in the number of required evaluations of $C$, which here is done by Algorithm 1. It is usually implicitly assumed that each evaluation is recursive, so that the total number of operations is also linear. This would not be achievable with methods that require many passes over the data, such as aPELT (Zhao and Yau, 2019) or even more complex detectors (Ma et al., 2020). Therefore, development of Algorithm 1 was essential to create the overlap detector.

While many simpler methods for classic changepoint detection are available, models incorporating the epidemic structure are more accurate in weak-signal settings, as seen in our simulations. Furthermore, the epidemic methods directly output periods of interest without manual post-processing. This is necessary, for example, in speech detection (Mesaros et al., 2017) or observatory data analysis (McNabb et al., 2004), where the identified segments are further processed automatically. However the major practical benefit of this framework is the ability to define and separate non-target segments, as used in this paper. The model may be extended to accommodate other features distinguishing different segment types as well.

We anticipate that the nuisance-signal separation will aid downstream processing, reducing the false alarm rate or the manual load if the detections are reviewed. Despite that, it is difficult to evaluate this benefit at present: while there are recent datasets prepared specifically for testing changepoint detection (Hocking et al., 2017; van den Burg and Williams, 2020), they are based on labelling all visually apparent changes. In future work, further application-specific comparisons could measure the impact of neutralising the nuisance process. 


\section{Bibliography}

Aminikhanghahi, S. and Cook, D. J. (2016) A survey of methods for time series change point detection. Knowledge and Information Systems, 51, 339-367.

Baranowski, R., Chen, Y. and Fryzlewicz, P. (2019) Narrowest-over-threshold detection of multiple change points and change-point-like features. Journal of the Royal Statistical Society: Series B (Statistical Methodology), 81, 649-672.

Baud, D., Qi, X., Nielsen-Saines, K., Musso, D., Pomar, L. and Favre, G. (2020) Real estimates of mortality following COVID-19 infection. The Lancet Infectious Diseases, 20, 773.

Bottou, L. (1998) Online algorithms and stochastic approximations. In Online Learning and Neural Networks (ed. D. Saad). Cambridge, UK: Cambridge University Press.

van den Burg, G. J. J. and Williams, C. K. I. (2020) An evaluation of change point detection algorithms. arXiv preprint.

Fisch, A. T. M., Eckley, I. A. and Fearnhead, P. (2018) A linear time method for the detection of point and collective anomalies. arXiv preprint.

Fryzlewicz, P. (2014) Wild binary segmentation for multiple change-point detection. The Annals of Statistics, 42, 2243-2281.

Hochenbaum, J., Vallis, O. S. and Kejariwal, A. (2017) Automatic anomaly detection in the cloud via statistical learning. arXiv preprint.

Hocking, T. D., Goerner-Potvin, P., Morin, A., Shao, X., Pastinen, T. and Bourque, G. (2017) Optimizing ChIP-seq peak detectors using visual labels and supervised machine learning. Bioinformatics, 33, 491-499.

Hocking, T. D., Rigaill, G., Fearnhead, P. and Bourque, G. (2018) Generalized functional pruning optimal partitioning (gfpop) for constrained changepoint detection in genomic data. arXiv preprint.

Jackson, B., Scargle, J., Barnes, D., Arabhi, S., Alt, A., Gioumousis, P., Gwin, E., San, P., Tan, L. and Tsai, T. T. (2005) An algorithm for optimal partitioning of data on an interval. IEEE Signal Processing Letters, 12, 105-108.

Jeng, X. J., Cai, T. T. and Li, H. (2010) Optimal sparse segment identification with application in copy number variation analysis. Journal of the American Statistical Association, 105, 1156-1166.

Killick, R., Fearnhead, P. and Eckley, I. A. (2012) Optimal detection of changepoints with a linear computational cost. Journal of the American Statistical Association, 107, 15901598.

Lau, T. S. and Tay, W. P. (2019) Quickest change detection in the presence of a nuisance change. IEEE Transactions on Signal Processing, 67, 5281-5296. 
Li, S., Cao, Y., Leamon, C., Xie, Y., Shi, L. and Song, W. (2016) Online seismic event picking via sequential change-point detection. In 2016 54th Annual Allerton Conference on Communication, Control, and Computing (Allerton). IEEE.

Ma, L., Grant, A. J. and Sofronov, G. (2020) Multiple change point detection and validation in autoregressive time series data. Statistical Papers, 61, 1507-1528.

Maidstone, R., Hocking, T., Rigaill, G. and Fearnhead, P. (2016) On optimal multiple changepoint algorithms for large data. Statistics and Computing, 27, 519-533.

McNabb, J. W. C., Ashley, M., Finn, L. S., Rotthoff, E., Stuver, A., Summerscales, T., Sutton, P., Tibbits, M., Thorne, K. and Zaleski, K. (2004) Overview of the BlockNormal event trigger generator. Classical and Quantum Gravity, 21, S1705-S1710.

Mesaros, A., Heittola, T., Diment, A., Elizalde, B., Shah, A., Vincent, E., Raj, B. and Virtanen, T. (2017) DCASE 2017 challenge setup: Tasks, datasets and baseline system. In Proceedings of the Detection and Classification of Acoustic Scenes and Events 2017 Workshop, 85-92.

Niu, Y. S., Hao, N. and Zhang, H. (2016) Multiple change-point detection: A selective overview. Statistical Science, 31, 611-623.

Page, E. S. (1954) Continuous inspection schemes. Biometrika, 41, 100.

Texier, G., Farouh, M., Pellegrin, L., Jackson, M. L., Meynard, J.-B., Deparis, X. and Chaudet, H. (2016) Outbreak definition by change point analysis: a tool for public health decision? BMC Medical Informatics and Decision Making, 16.

Truong, C., Oudre, L. and Vayatis, N. (2020) Selective review of offline change point detection methods. Signal Processing, 167, 107299.

Yuan, J., Li, M., Lv, G. and Lu, Z. K. (2020) Monitoring transmissibility and mortality of COVID-19 in Europe. International Journal of Infectious Diseases, 95, 311-315.

Zhang, Y., Liu, T., Meyer, C. A., Eeckhoute, J., Johnson, D. S., Bernstein, B. E., Nussbaum, C., Myers, R. M., Brown, M., Li, W. and Liu, X. S. (2008) Model-based analysis of ChIPseq (MACS). Genome Biology, 9, R137.

Zhao, Z. and Yau, C. Y. (2019) Alternating pruned dynamic programming for multiple epidemic change-point estimation. arXiv preprint.

Zheng, C., Eckley, I. A. and Fearnhead, P. (2019) Consistency of a range of penalised cost approaches for detecting multiple changepoints. arXiv preprint. 


\section{Supplementary material}

\section{A Proof of Theorem 1 (Convergence of Algorithm 1)}

Bottou (1998) analyses the case of an online algorithm iteratively minimising some function $f(x, w)$ (where $x$ represents the complete data and $w$ the parameters). Data points $\left\{x_{t}\right\}$ arrive sequentially, and at each iteration an estimate $w_{t}$ of the location of the minimum $w^{*}$ is obtained using some update function $H(x, w)$ and learning rate $\gamma_{t}$ as:

$$
w_{t+1}=w_{t}-\gamma_{t} H\left(x_{t+1}, w_{t}\right) .
$$

This updating mechanism gives rise to stochastic gradient descent if $\mathbb{E} H\left(x_{t+1}, w_{t}\right)=$ $\nabla_{w} f(x, w)$, but for the following argument this is not required.

To make the link with Algorithm 1 explicit, the update equation applied by this algorithm can be written as:

$$
w_{t+1}=w_{t}+\gamma_{t}\left(x_{t+1}-w_{t}\right)
$$

Then $w^{*}=\theta_{0}$ (i.e., the background mean that is to be estimated), and we ask whether the sequence of updates converges $w_{t} \rightarrow w^{*}$. It was shown by Bottou (1998) that this occurs almost surely if the following three conditions are met:

1. "convexity" - a single optimum $w^{*}$ exists and the expected value of the updates always points towards it:

$$
\forall \epsilon>0, \inf _{\left(w-w^{*}\right)^{2}>\epsilon}\left(w-w^{*}\right) \mathbb{E} H(x, w)>0 ;
$$

2. learning rate convergence:

$$
\sum_{i=1}^{\infty} \gamma_{t}=\infty, \sum_{i=1}^{\infty} \gamma_{t}^{2}<\infty
$$

3. bounded variance of the updates:

$$
\mathbb{E} H(x, w)^{2} \leq A+B\left(w-w^{*}\right)^{2}, A, B \geq 0 .
$$

Thus, proof of convergence of our algorithm reduces to showing that these requirements are satisfied. We start with the assumption that the global mean of segment points is also $\theta_{0}$, and then relax this requirement. 
The following lemma will be needed:

Lemma 1. Let $f$ be a unimodal pdf, symmetric around a point $\mu$ (so that $f\left(x_{1}\right)<$ $f\left(x_{2}\right)$ when $x_{1}<x_{2} \leq \mu$ and $f\left(x_{1}\right)>f\left(x_{2}\right)$ when $\left.\mu \leq x_{1}<x_{2}\right)$, such as a Gaussian. Consider a truncated random variable $X$ with $p d f$ :

$$
g(x)= \begin{cases}0 & \text { if } x<m-a \\ \frac{f(x)}{P(m-a \leq x \leq m+a)} & \text { if } m-a \leq x \leq m+a \\ 0 & \text { if } x>m+a\end{cases}
$$

for some $a>0, m$. Then for any $\epsilon>0, \inf _{|m-\mu|>\epsilon}(\mathbb{E} X-m)(\mu-m)>0$.

Proof.

$$
\begin{aligned}
\mathbb{E} X-m & =\int_{m-a}^{m+a}(x-m) f(x) d x \\
& =\int_{-a}^{0} y f(y+m) d y+\int_{0}^{a} y f(y+m) d y \\
& =\int_{0}^{a} y(f(m+y)-f(m-y)) d y .
\end{aligned}
$$

When $m+a<\mu, f$ is increasing throughout the integration range, and $\mathbb{E} X-m>0$; the opposite is true for $m-a>\mu$. If $m-a<m<\mu<m+a$, split the integral in (S16) as:

$$
\mathbb{E} X-m=\int_{0}^{\mu-m} y(f(m+y)-f(m-y)) d y+\int_{\mu-m}^{a} y(f(m+y)-f(m-y)) d y .
$$

The first integral covers the range where $f$ is increasing, and thus is positive. Since $\mu-m>0,|m+y-\mu|<|m-y-\mu|$ for $y>0$, and $f(m+y)>f(m-y)$ by symmetry of $f$ around $\mu$ and monotonicity, so the second interval is positive as well. Similarly, $\mathbb{E} X-m<0$ for $m-a<\mu<m<m+a$.

\section{When the global mean of segments matches the background mean}

Consider the case that the background points are independent draws from $\mathscr{N}\left(w^{*}, \sigma^{2}\right)$, and the points within each segment are $\mathscr{N}\left(\theta_{i}, \sigma^{2}\right)$, with $\sigma^{2}$ known, and $\theta_{i}$ such that the marginal pdf $f_{S}$ is symmetric around $w^{*}$. Let $w_{t}$ be the value of the background mean estimated by Algorithm 1 after processing $t$ data points. In this case $w_{t} \stackrel{\text { a.s. }}{\longrightarrow} w^{*}$. 
Proof. Denote the true class of the next data point $x_{t+1}$ by $\delta_{t+1}$ ( 1 for background points, 0 for signal). Algorithm 1 estimates this as:

$$
\hat{\delta}_{t+1}= \begin{cases}1 & \text { if } F(t)+C^{0}\left(x_{t+1} ; w_{t}\right)<\min _{1 \leq k \leq l} F(t+1-k)+C\left(x_{t+2-k: t+1}\right)+\beta \\ 0 & \text { otherwise. }\end{cases}
$$

Initially, assume for simplicity that the true maximum segment length is 1 (and so only $k=1$ is tested). When $\hat{\delta}_{t+1}=1$, the background estimate is updated as:

$$
w_{t+1}=w_{t}+\frac{1}{\sum_{i=1}^{t} \hat{\delta}_{i}+1}\left(x_{t+1}-w_{t}\right)
$$

(otherwise $\left.w_{t+1}=w_{t}\right)$. So $\gamma_{t}=1 /\left(\sum_{i=1}^{t} \hat{\delta}_{i}+1\right)$, and hence the learning rate convergence conditions (S14) are satisfied.

Substituting in the costs based on a one-dimensional Gaussian pdf $\phi, \hat{\delta}_{t+1}=1$ if:

$$
\begin{aligned}
& -\log \phi\left(x_{t+1} ; w_{t}, \sigma^{2}\right)<-\log \phi\left(x_{t+1} ; x_{t+1}, \sigma^{2}\right)+\beta \\
\Rightarrow & \frac{1}{\sigma \sqrt{2 \pi}} \exp \frac{-\left(x_{t+1}-w_{t}\right)^{2}}{2 \sigma^{2}}>\frac{1}{\sigma \sqrt{2 \pi}} e^{-\beta} \\
\Rightarrow & \left|x_{t+1}-w_{t}\right|<\sqrt{2 \beta \sigma^{2}} \\
\Rightarrow & x_{t+1} \in\left(w_{t}-\sqrt{2 \beta \sigma^{2}} ; w_{t}+\sqrt{2 \beta \sigma^{2}}\right) .
\end{aligned}
$$

By construction, the distribution of true segment data points $f\left(x_{t+1} \mid \delta_{t+1}=0\right)$ is symmetric with mean $w^{*}$, and the same applies to the background point distribution. Thus, the overall distribution of the points used to update the $w_{t}$ estimate is a truncation of a symmetric unimodal distribution. In the present case, it is a truncated normal with limits $\left(w_{t}-\sigma \sqrt{2 \beta}, w_{t}+\sigma \sqrt{2 \beta}\right)$, based on (S17); more generally it is a truncated variant of the parent distribution with symmetric limits of the form $w_{t} \pm a$, and parent mean $w^{*}$ (that the acceptance set is an interval follows from the unimodality of $f$ ).

This means that $f\left(x_{t+1} \mid \hat{\delta}_{t+1}=1\right)$ satisfies the requirements for Lemma 1 with $\mu=w^{*}$, which implies the "convexity" condition (S13):

$$
\inf _{\left(w-w^{*}\right)^{2}>\epsilon}\left(w-w^{*}\right) \mathbb{E} H\left(x_{t+1}, w\right)=\inf _{\left(w-w^{*}\right)^{2}>\epsilon}\left(w-w^{*}\right)\left(w-\mathbb{E}\left(x_{t+1} \mid \hat{\delta}_{t+1}=1\right)\right)>0
$$

Following a similar approach - conditioning on $\delta_{t+1}-$ and using the law of total 
variance it can be shown that the variance of $\mathbb{E}\left(w-x_{t+1}\right)$ is finite, as required for the condition (S15), and so $w_{t} \stackrel{\text { a.s. }}{\longrightarrow} w^{*}$.

Remark. So far, we assumed that segment length $k=1$. If segments occur and are tested in non-overlapping windows of any fixed size $k \geq 2$, the result is similar: $\forall j \in[t-k+1 ; t], \hat{\delta}_{j+1}=1$ if:

$$
-\sum_{i=t-k+1}^{i=t} \log \phi\left(x_{i+1} ; w_{t-k}, \sigma^{2}\right)<-\sum_{i=t-k+1}^{i=t} \log \phi\left(x_{i+1} ; \bar{x}_{k}, \sigma^{2}\right)+\beta
$$

where $\bar{x}_{k}=\sum_{i=t-k+1}^{t} x_{i+1} / k$. This can be expressed as truncation limits for accepted $\bar{x}_{k}$, analogously to (S17):

$$
\begin{aligned}
\beta & >-\sum_{i=t-k+1}^{t} \log \phi\left(x_{i+1} ; w_{t-k}, \sigma^{2}\right)+\sum_{i=t-k+1}^{i=t} \log \phi\left(x_{i+1} ; \bar{x}_{k}, \sigma^{2}\right) \\
& =\sum_{i=t-k+1}^{t} \frac{\left(x_{i+1}-w_{t-k}\right)^{2}}{2 \sigma^{2}}-\frac{\left(x_{i+1}-\bar{x}_{k}\right)^{2}}{2 \sigma^{2}} \\
& =\frac{1}{2 \sigma^{2}} \sum_{i=t-k+1}^{t}\left(w_{t-k}-\bar{x}_{k}\right)^{2} \\
& \Rightarrow \sqrt{2 \beta \sigma^{2} / k}>\left|w_{t-k}-\bar{x}_{k}\right| .
\end{aligned}
$$

The pdf of $\bar{x}_{k}$ is a $k$-fold convolution of $f(x)$. Since $f$, as shown earlier, is symmetric unimodal, so is their convolution, and hence the distribution of $\bar{x}_{k}$, with a mean $\mu=w^{*}$ (Purkayastha, 1998). In the special case when $f$ is the normal pdf, this can also be shown directly from Gaussian properties. Then Lemma 1 implies condition (S13), and the rest of the proof follows as before.

When all segment positions (overlapping or not) are tested, the background point acceptance rule is:

$\hat{\delta}_{t}=1$ if $x_{t} \in \bigcap_{1 \leq k \leq l} S_{k}$, with $S_{k}=\left\{x_{t}: F(t-1)+C^{0}\left(x_{t}\right)<F(t-k)+C\left(x_{t-k+1: t}\right)+\beta\right\}$.

As demonstrated earlier, $S_{1}=\left(w_{t}-\sqrt{2 \beta \sigma^{2}}, w_{t}+\sqrt{2 \beta \sigma^{2}}\right)$. Define three sets of $x_{t}$ based on which rules they pass: $X_{1}=x_{t} \in S_{1}, X_{a}=x_{t} \in S_{1} \cap S_{\geq 2}, X_{r}=x_{t} \in S_{1} \backslash S_{\geq 2}$, and let $P_{a}$ and $P_{r}$ be the probabilities of the corresponding $x_{t}$ sets. Clearly, $X_{1}=$ $X_{a} \cup X_{r}$. We are interested in the mean of the points accepted as background, i.e. $\mathbb{E} X_{a}$. Assume w.l.o.g. $\mu=0, \sigma=1, w_{t}>\mu$, as the other case is symmetric. We will now show that for sufficiently large $n, \mathbb{E} X_{a}<w_{t}$, satisfying (S13). 
Using the conditional mean formula:

$$
\begin{aligned}
& \mathbb{E} X_{1}=P_{r} \mathbb{E} X_{r}+P_{a} \mathbb{E} X_{a} \\
& \mathbb{E} X_{a}=\mathbb{E} X_{1} /\left(1-P_{r}\right)-P_{r} \mathbb{E} X_{r} /\left(1-P_{r}\right) .
\end{aligned}
$$

Assume for now $\mathbb{E} X_{r}=\mu=0$. Then, to obtain $\mathbb{E} X_{a}<w_{t}$, we need:

$$
\mathbb{E} X_{1} / w_{t}<1-P_{r}
$$

Denote $p=\sqrt{2 \beta}$, which is an increasing function of $n$, and consider the growth of both sides of (S20) as $n$ increases. For the Gaussian or other distributions in the exponential family with mean $\mu$, truncated to a symmetric region $(-a, a)$, it is known that $\operatorname{Var}(X \mid S ; \mu)=\frac{d}{d \mu} \mathbb{E}(X \mid S ; \mu)$ (Zidek and van Eeden, 2003). Then (denot$\left.\operatorname{ing} S_{1}^{\prime}=(-p ;+p)\right)$ :

$$
\begin{aligned}
\mathbb{E} X_{1} & =w_{t}+\mathbb{E}\left(X \mid S_{1}^{\prime} ;-w_{t}\right) \\
& =w_{t}+\mathbb{E}\left(X \mid S_{1}^{\prime} ; 0\right)+\int_{0}^{w_{t}} \frac{d \mathbb{E}\left(X \mid S_{1}^{\prime} ;-a\right)}{d a} d a \\
& =w_{t}+0-\int_{0}^{w_{t}} \frac{d \mathbb{E}\left(X \mid S_{1}^{\prime} ; a\right)}{d a} d a \\
& <w_{t}-w_{t} \min _{0 \leq a \leq w_{t}} \operatorname{Var}\left(X \mid S_{1}^{\prime} ; a\right) .
\end{aligned}
$$

Hence:

$$
\mathbb{E} X_{1} / w_{t}<1-\operatorname{Var}\left(X \mid S_{1}^{\prime} ; w_{t}\right)=1-\int_{w_{t}-p}^{w_{t}+p} x^{2} f(x) d x,
$$

where $f(x)$ is the pdf of $x_{t}$ given $x \in S_{1}$. Hence, this side grows with $p$ as $-x^{2} f(x)$.

To analyse $P_{r}$, we first simplify the background acceptance condition. For any $k \geq 2$, by definition of $F$ and additivity of $C$, we have:

$$
\begin{aligned}
F(t-k)+C\left(x_{t-k+1: t}\right)+\beta & =F(t-k)+C\left(x_{t-k+1: t-1} ; \bar{x}_{k}\right)+C\left(x_{t} ; \bar{x}_{k}\right)+\beta \\
& =F(t-k)+C\left(x_{t-k+1: t-1} ; \bar{x}_{-t}\right)+(k-1) d\left(\bar{x}_{k}, \bar{x}_{-t}\right)+d\left(\bar{x}_{k}, x_{t}\right)+\beta \\
& =F(t-1)+A(t-1)+(k-1) d\left(\bar{x}_{k}, \bar{x}_{-t}\right)+d\left(\bar{x}_{k}, x_{t}\right),
\end{aligned}
$$

where $d$ is some distance function, $\bar{x}_{-t}$ is the mean of points $x_{t-k+1: t-1}$, and $A(t) \geq 0$ is a constant depending only on $x_{1: t}$. 
It is also helpful to note that $F(t-1) \leq F(t-k)+C^{0}\left(x_{t-k+1: t-1}\right)$, hence:

$$
\begin{aligned}
A(t-1) & =F(t-k)+C\left(x_{t-k+1: t-1} ; \bar{x}_{-t}\right)+\beta-F(t-1) \\
& \geq C\left(x_{t-k+1: t-1} ; \bar{x}_{-t}\right)+\beta-C^{0}\left(x_{t-k+1: t-1}\right) \\
& \geq \beta-(k-1) d\left(\bar{x}_{-t}, w_{t-1}\right) .
\end{aligned}
$$

This corresponds to the case when all $x_{t-k+1: t-1}$ were identified as background.

Using the Gaussian cost, i.e. $d(a, b)=(a-b)^{2} / 2$, and recursive formula for the mean, the acceptance condition for $k$ becomes:

$$
\begin{aligned}
& F(t-1)+C^{0}\left(x_{t}\right)<F(t-1)+A(t-1)+\frac{k-1}{2 k^{2}}\left(x_{t}-\bar{x}_{-t}\right)^{2}+\frac{(k-1)^{2}}{2 k^{2}}\left(x_{t}-\bar{x}_{-t}\right)^{2} \\
& \Rightarrow\left(x_{t}-w_{t-1}\right)^{2}<2 A(t-1)+\frac{k-1}{k}\left(x_{t}-\bar{x}_{-t}\right)^{2} .
\end{aligned}
$$

By substituting in the value of $A(t-1)$ from (S21), we obtain the following lower bound for $P\left(x \in S_{k} \mid x\right)$ :

$$
\begin{aligned}
P\left(x \in S_{k} \mid x\right) & \geq P\left(\left(x_{t}-w_{t-1}\right)^{2}<2 \beta-(k-1)\left(\bar{x}_{-t}-w_{t-1}\right)^{2}+\frac{k-1}{k}\left(x_{t}-\bar{x}_{-t}\right)^{2}\right) \\
& =P\left(\left(x_{t}-w_{t-1}\right)^{2}-\frac{k-1}{k}\left(x_{t}-\bar{x}_{-t}\right)^{2}+(k-1)\left(\bar{x}_{-t}-w_{t-1}\right)^{2}<2 \beta\right) \\
& \geq P\left(\left(x_{t}-w_{t-1}\right)^{2}+(k-1)\left(\bar{x}_{-t}-w_{t-1}\right)^{2}<2 \beta\right) \\
& \left.\geq 1-\mathbb{E}\left(\left(x_{t}-w_{t-1}\right)^{2}\right)+(k-1)\left(\bar{x}_{-t}-w_{t-1}\right)^{2}\right) /(2 \beta) \\
P\left(x \notin S_{k} \mid x\right) & \left.\leq \mathbb{E}\left(\left(x_{t}-w_{t-1}\right)^{2}\right)+(k-1)\left(\bar{x}_{-t}-w_{t-1}\right)^{2}\right) /(2 \beta) .
\end{aligned}
$$

Thus, we have the following bound for $P_{r}$ at any $k$ :

$$
\begin{aligned}
P_{r} & =\int_{w_{t}-p}^{w_{t}+p} P\left(x \notin S_{k} \mid x\right) f(x) d x \\
& \leq \int_{w_{t}-p}^{w_{t}+p} O\left(x^{2} / p^{2}\right) f(x) d x .
\end{aligned}
$$

Therefore, as $N$ increases, $1-P_{r}$ grows faster than $1-\mathbb{E} X_{1} / w_{t}=1-\int_{w_{t}-p}^{w_{t}+p} x^{2} f(x) d x$. This means that $\exists p_{0}$, and thus $\exists n_{0}$, such that for $n>n_{0}$, and thus $p>p_{0}$, (S20) holds.

So far we assumed $\mathbb{E} X_{r}=\mu$. Clearly, for larger values of $\mathbb{E} X_{r}, \mathbb{E} X_{a}$ is even smaller and (S13) is satisfied.

For the case when $\mathbb{E} X_{r}<\mu$, consider the worst case scenario $\mathbb{E} X_{r}=w_{t}-p$ (this 
is the bound to $X_{1}$, and thus to $X_{r}$, imposed by $S_{1}$ ). Similarly to (S19), we need:

$$
\begin{aligned}
& \mathbb{E} X_{a}<w_{t} \\
\Rightarrow & \mathbb{E} X_{1} /\left(1-P_{r}\right)-P_{r}\left(w_{t}-p\right) /\left(1-P_{r}\right)<w_{t} \\
\Rightarrow & \mathbb{E} X_{1} / w_{t}-P_{r}+P_{r} p / w_{t}<1-P_{r} \\
\Rightarrow & P_{r} p / w_{t}<1-\mathbb{E} X_{1} / w_{t} \\
\Rightarrow & P_{r} p / w_{t}<\int_{w_{t}-p}^{w_{t}+p} x^{2} f(x) d x .
\end{aligned}
$$

However, based on (S23), $P_{r} p / w_{t} \leq \int_{w_{t}-p}^{w_{t}+p} O\left(x^{2} / p\right) f(x) d x$, so again $\exists n>n_{0}$ such that $\mathbb{E} X_{a}<w_{t}$, and condition (S13) holds.

And so overall $\mathbb{E}\left(x_{t} \mid \hat{\delta}_{t}=1\right)$ satisfies condition (S13). Since the distribution of accepted $x_{t}$ still has bounded support imposed by $S_{1}$, condition (S15) still holds, and the learning rate condition (S14) holds as before, implying convergence.

\section{When the global mean of segments does not match the background mean}

Consider now that the overall mean of segment points $\mu_{S}=\int_{-\infty}^{\infty} x f_{S}(x) d x \neq w^{*}$, in particular $\mu_{S}>w^{*}$. Then if $w^{*}<w_{t}<\mu_{S}$, any segment points that were misclassified as background will (on average) push the estimates away from the background mean, in violation of the "convexity" condition (S13).

We assume that each segment point is followed by no less than $n$ background points. Then, as $n \rightarrow \infty, w_{t} \stackrel{a . s}{\longrightarrow} w^{*}$. For every finite $n, \exists \epsilon>0$ such that $P\left(\left|w_{t}-w^{*}\right|>\right.$ $\epsilon)=0$.

Proof. Suppose that a misclassification at time $T$ is followed by $n$ correctly classified background points: $\delta_{T}=0, \delta_{t}=1$ for $t \in[T+1 ; T+n], \hat{\delta}_{t}=1$ for $t \in[T ; T+n]$. For the points $t \in[T+1 ; T+n]$, almost sure convergence of $w_{t}$ was established above, i.e. for all $\epsilon>0$, there exists a $t_{0}$ such that $\forall t: n \geq t \geq t_{0}, P\left(\left|w_{T+t}-w^{*}\right|<\epsilon\right)=1$. Therefore, given $n \geq t_{0}$ :

$$
\begin{aligned}
& P\left(\left|w_{T+n}-w^{*}\right|<\left|w_{T-1}-w^{*}\right|\right)=1 \\
& \Rightarrow\left\{\begin{array}{l}
P\left(w_{T+n}-w_{T-1}<0\right)=1, \text { if } w_{T-1}-w^{*}>0 \\
P\left(w_{T+n}-w_{T-1}>0\right)=1, \text { if } w_{T-1}-w^{*}<0
\end{array}\right. \\
& \Rightarrow \inf _{w_{T-1} \neq w^{*}}\left(w_{T-1}-w^{*}\right) \mathbb{E}\left(w_{T+n}-w_{T-1}\right)<0 .
\end{aligned}
$$


Indexing the segment-background cycles by $i$, denote the first estimate of that segment by $w_{i}^{\prime}$, so the set of these estimates are:

$$
\left\{w_{i}^{\prime}\right\}=\left\{w_{1}, \ldots, w_{T-2-n}, w_{T-1}, w_{T+n}, w_{T+1+2 n}, \ldots\right\} .
$$

The elements of this sequence can be expressed recursively as:

$$
w_{i+1}^{\prime}=w_{i}^{\prime}-\gamma_{i}^{\prime} H^{\prime}\left(\left\{x_{i}^{\prime}\right\}, w_{i}^{\prime}\right)
$$

with $\left\{x_{i}^{\prime}\right\}=\left\{x_{t}: i(n+1) \leq t \leq i(n+1)+n\right\}$.

From (S24), $\mathbb{E}\left(w_{i}^{\prime}-w_{i+1}^{\prime}\right)=\gamma_{i}^{\prime} \mathbb{E} H^{\prime}\left(\left\{x_{i}^{\prime}\right\}, w_{i}^{\prime}\right)$ is "convex" as defined in (S13), and because $\gamma_{i}^{\prime}>0$ so is $\mathbb{E} H^{\prime}\left(\left\{x_{i}^{\prime}\right\}, w_{i}^{\prime}\right)$.

Let $\gamma_{i}^{\prime}=\frac{1}{i(n+1)+1}$. Then:

$$
\begin{aligned}
H^{\prime}\left(\left\{x_{i}^{\prime}\right\}, w_{i}^{\prime}\right) & =\sum_{t=i(n+1)}^{i(n+1)+n} \gamma_{t}\left(w_{t-1}-x_{t}\right) / \gamma_{i}^{\prime} \\
& =\sum_{t=i(n+1)}^{i(n+1)+n} \frac{i(n+1)+1}{t+1}\left(w_{t-1}-x_{t}\right) \\
& <(n+1)\left(w_{t-1}-x_{t}\right) .
\end{aligned}
$$

So for $n<\infty$, conditions (S14)-(S15) are satisfied as well, and $w_{i}^{\prime} \stackrel{\text { a.s. }}{\rightarrow} w^{*}$. (When $n \rightarrow \infty$, the convergence conditions are satisfied directly without using the sequence $\left\{w_{i}^{\prime}\right\}$.)

\section{Martingale Approach}

We can also describe the update process over the background points using martingales. The algorithm estimates are random variables $w_{t}$; let $\left\{\mathscr{W}_{t}\right\}$ be the sequence of $\sigma$-algebras such that for each $t, w_{t}$ is measurable with respect to $\mathscr{W}_{t}$. Using Lemma 1 , and assuming $w^{*}<w_{t}$ again, within each cycle the estimates comprise a supermartingale $\mathbb{E}\left(w_{t+1} \mid \mathscr{W}_{t}\right)<w_{t}$ over the points $T \leq t<\min \left(T+n, F H T_{w}\left(w^{*}\right)\right)$, here $F_{H T}(a)=\inf \left\{t: x_{t} \leq a\right\}$ is the first hitting time of the process realisation $\left\{x_{t}\right\}$ to value $a$.

Consider again the problematic case when the global mean does not match the background mean and misclassification pushes the estimate away from the background mean, i.e. $w^{*}<w_{T-1}<w_{T}<\mu_{S}$. In order for $w_{i}^{\prime}$ to converge, we need the perturbed estimates to return to a value below $w_{T-1}$ in each cycle. At the extremes, 
we have:

$$
\begin{array}{ll}
F_{H} T_{w}\left(w_{T}\right)=T & \text { starting position } \\
F_{H T}\left(w_{T-1}\right)<\infty & \text { for sufficiently large } n \text {, because } w_{t} \stackrel{\text { a.s. }}{\longrightarrow} w^{*} .
\end{array}
$$

Clearly, $F H T_{w}\left(w_{T-1}\right) \leq F H T_{w}\left(w^{*}\right)$. However, the number of background points $n$ required to satisfy $F_{H} T_{w}\left(w_{T-1}\right)<T+n$ will depend on five factors: the distribution $f_{B}$ and penalty $p$ (since they determine the distribution of update values $H$ ), the size of estimated background set at time $T$ (as it determines the relevant $\gamma_{t}$ ), and $w_{T-1}$ and $w_{T}$.

In practice, $n$ is bounded by the available data, so there is a non-zero probability that, over the segment-background cycles indexed by $i$ :

$$
\max _{i} F_{w} T_{w}\left(w_{T_{i}-1}\right)>T_{i}+n
$$

In that case, define $b=\min \left\{a: \forall i, F H T_{w}(a) \leq T_{i}+n\right\}$; the final estimate of $w_{i}^{\prime}$ will be bound by $\left[w^{*}, b\right]$. As $n$ increases, $P\left(\left|w^{*}-b\right|>\epsilon\right) \rightarrow 0$ for any $\epsilon>0$.

Similar reasoning applies when $\mu_{S}<w_{T}<w_{T-1}<w^{*}$.

\section{B Proof of Theorem 2 (Consistency)}

Proof. Some general consistency results for changepoint detection by penalised cost methods are given in Fisch et al. (2018). In particular, an equivalent of our Theorem 2 is established for any algorithm that detects changepoints by exactly minimising a $\operatorname{cost} F\left(n ;\left\{s_{i}, e_{i}\right\}, \theta, \hat{\mu}, \hat{\sigma}\right)$, where $\hat{\bullet}$ marks robust estimates of background parameters. While the original statement uses the median and interquartile range of $x_{0: n}$ for $\hat{\mu}$ and $\hat{\sigma}$, the proof only requires that the estimates satisfy certain upper bounds on deviations from the true values. Therefore, we will first show that the online estimates produced by Algorithm 1 are within these bounds, and then follow the rest of the proof from Fisch et al. (2018).

Noting again that Algorithm 1 is effectively a stochastic gradient descent procedure, with each data point seen precisely once, we can use the error bound on estimates produced by such algorithms as provided in Theorem 7.5 of Harvey et al. (2019):

Theorem 3 ((Harvey et al., 2019)). Let function $f(w)$ be 1-strongly convex and 1-Lipschitz. A stochastic gradient algorithm for minimising this function runs for $T$ cycles, and at each cycle updates the estimate as in (S12) with $\gamma_{t}=1 / t, \mathbb{E} H=\nabla f(w)$. 
Then:

$$
P\left(\left\|w_{t}-w^{*}\right\|^{2} \leq O\left(\frac{\log (1 / \delta)}{t}\right)\right) \geq 1-\delta
$$

Using $\delta=n^{-\epsilon}$, and assuming without loss of generality that $\sigma_{0}=1$, we can establish an upper bound on the error of background parameters estimated by Algorithm 1 after $n$ cycles:

$$
\begin{aligned}
& P\left(\left(\hat{\mu}-\mu_{0}\right)^{2} \leq O\left(\frac{\log \left(n^{\epsilon}\right)}{n}\right)\right)=P\left(\left|\hat{\mu}-\mu_{0}\right| \leq O\left(\sqrt{\epsilon} \sqrt{\frac{\log n}{n}}\right)\right) \geq 1-n^{-\epsilon} \\
& P\left(\left|\hat{\sigma}^{2}-\sigma_{0}^{2}\right| \leq O\left(\sqrt{\epsilon} \sqrt{\frac{\log n}{n}}\right)\right) \geq 1-n^{-\epsilon} .
\end{aligned}
$$

Application of Boole's inequality leads to:

$$
P\left(\left|\hat{\mu}-\mu_{0}\right| \leq D_{1} \sigma_{0} \sqrt{\frac{\log (n)}{n}},\left|\frac{\hat{\sigma}^{2}}{\sigma_{0}^{2}}-1\right| \leq D_{2} \sqrt{\frac{\log (n)}{n}}\right) \geq 1-C_{1} n^{-\epsilon}
$$

for some constants $C_{1}, D_{1}, D_{2}$ and sufficiently large $n$. Since the objective function $f$ in our algorithm is the Gaussian log-likelihood (i.e., the updates $\mathbb{E} H$ approximate its gradient), for any given segmentation it is 1-strongly convex. For other functions, overall consistency can still be achieved similarly, but the convergence rate may be slower than $n^{-\epsilon}$.

Having established the bound on estimate errors, we can use Lemma 9 from Fisch et al. (2018) and the proof method reported there.

First, introduce an event $E$ based on a combination of bounds limiting the behaviour of Gaussian data $x_{1: n}$, which for any $\epsilon>0$, occurs with probability $P(E)>1-C_{2} n^{-\epsilon}$, with some constant $C_{2}$ and sufficiently large $n$ (Lemmas 1 and 2 in Fisch et al. (2018)). Conditional on this event, the following lemma holds for the epidemic cost $F$ defined as in the main text, eq. 5:

Lemma 2 ((Fisch et al., 2018)). Let $\{\tau\}$ be the set of true segment positions $\left\{\left(s_{i}, e_{i}\right)\right\}$, and $\theta$ the vector of true segment means. Assume $E$ holds, and some $\hat{\mu}, \hat{\sigma}$ are available for which the event in (S25) holds. Then, there exist constants $C_{3}$ and $n_{1}$ such that when $n>n_{1}$,

$$
F(n ;\{\tau\}, \theta, \hat{\mu}, \hat{\sigma})-F\left(n ;\{\tau\}, \theta, \mu_{0}, \sigma_{0}\right)<C_{4} \log n
$$

This lemma, together with results established for classical changepoint detection, can be used to show that the cost of any inconsistent solution will exceed the 
cost based on true segment positions and parameters (Proposition 8 in Fisch et al. (2018)):

Proposition 2 ((Fisch et al., 2018)). Define $\left\{\tau^{\prime}\right\}$ to be any set of segments $\left\{\left(s_{i}, e_{i}\right)\right\}$ that are not consistent (as defined in the main text, eq. 7). Let $\tilde{\theta}=\operatorname{argmin}_{\theta} F(n ; \theta)$ be the parameters estimated by minimising the cost for a given segmentation (i.e. the vector of means and/or variances of $x_{s_{i}: e_{i}}$ for each $i$ ). Assume E holds. Then there exist constants $C_{4}$ and $n_{2}$ such that, when $n>n_{2}$ :

$$
F\left(n ;\left\{\tau^{\prime}\right\}, \tilde{\theta}, \hat{\mu}, \hat{\sigma}\right) \geq F(n ;\{\tau\}, \theta, \hat{\mu}, \hat{\sigma})+C_{3} \log (n)^{1+\delta / 2}
$$

See the original publication for a detailed proof of these results.

Finally, for a given set of changepoints, using fitted maximum-likelihood parameters by definition results in minimal cost:

$$
F(n ;\{\tau\}, \theta, \hat{\mu}, \hat{\sigma}) \geq F(n ;\{\tau\}, \tilde{\theta}, \hat{\mu}, \hat{\sigma})
$$

Thus, when Proposition 2 holds, we have:

$$
F\left(n ;\left\{\tau^{\prime}\right\}, \tilde{\theta}, \hat{\mu}, \hat{\sigma}\right)>F(n ;\{\tau\}, \tilde{\theta}, \hat{\mu}, \hat{\sigma})
$$

and an exact minimisation algorithm will always find a solution in the consistent set. The overall probability of the events required for Proposition 2 is a combination of $P(E)$, established before, and (S25), which by Boole's inequality is:

$$
P>1-C_{5} n^{-\epsilon}
$$

for any $\epsilon>0, n>n_{3}$ and some constants $n_{3}, C_{5}$.

\section{Pruning of Algorithm 1}

Much of the improvement in performance of change estimation algorithms comes from pruning of the search space. So far, the cost function was only required to be block-additive (see (2.2) in the maintext). Most lihelihood-based costs are also superadditive over points: for all $a \leq b<c$,

$$
C\left(x_{a: c}\right) \geq C\left(x_{a: b}\right)+C\left(x_{b+1: c}\right) .
$$


In that case, standard pruning (Killick et al., 2012) can be applied to Algorithm 1, and the resulting solution will still be optimal, as shown in Proposition 3.

To implement this, the search set for $F_{S}$ in step 4 would be changed to:

$$
F_{S}=\min _{t^{\prime} \in K}\left(F\left(t^{\prime}\right)+C\left(x_{t^{\prime}+1: t}\right)+\beta\right)
$$

with $K$ initialized to $\{0\}$ and updated with a new step 11a:

$$
\text { 11a. } K=K \cap\left\{s: F(s)+C\left(x_{s+1: t}\right)<F(t), t+1-l \leq s<t\right\} \cup\{t\} \text {. }
$$

Proposition 3. Assume a cost function $C$ such that (S26) applies. If, for some $s>t-l$ :

$$
F(s)+C\left(x_{s+1: t}\right) \geq F(t),
$$

then $s+1$ will not be a segment start point in the optimal solution, and can be excluded from consideration for all subsequent $t^{\prime}>t$.

Proof. For all $t^{\prime} \leq s+l$, the proof applies as for other pruned algorithms (Killick et al., 2012):

$$
F(t)+C\left(x_{t+1: t^{\prime}}\right)+\beta \leq F(s)+C\left(x_{s+1: t}\right)+C\left(x_{t+1: t^{\prime}}\right)+\beta \leq F(s)+C\left(x_{s+1: t^{\prime}}\right)+\beta .
$$

For $t^{\prime}>s+l$, segment $\left(s+1, t^{\prime}\right)$ will exceed the length constraint and thus cannot be part of the final segmentation.

\section{Proof of Proposition 1 (Pruning of Algorithm 2)}

Proof. Denote the true start and end of a nuisance segment as $s_{j}, e_{j}$. Consider the case when $s_{j} \in(t-A(n) ; t]$. Pruning at time $t$ will not remove this point (i.e. $\left.s_{j} \notin \boldsymbol{k}_{p r, t}\right)$ iff:

$$
C^{0}\left(x_{t-A(n): s_{j}-1}\right)+C^{N}\left(x_{s_{j}: t}\right)<C^{0}\left(x_{t-A(n): m-1}\right)+C^{N}\left(x_{m: t}\right)+\alpha \log (n)^{1+\delta}
$$

with $m$ such that the right hand side is minimised and $m \neq s_{j}$.

Denote by $C\left(x_{a: b} ; \hat{\mu}, \hat{\sigma}\right)$ the Gaussian cost calculated with MLE estimates of the parameters (i.e. mean and variance of $\left.x_{a: b}\right)$. Note that since $C^{0}\left(x_{a: b}\right)=C\left(x_{a: b} ; \mu_{0}, \sigma_{0}\right)$ 
and $C^{N}\left(x_{a: b}\right)=C\left(x_{a: b} ; \hat{\mu}, \sigma_{N}\right)$, the required event to preserve $s_{j}$ can be stated as

$$
\begin{aligned}
& C\left(x_{t-A(n): s_{j}-1} ; \mu_{0}, \sigma_{0}\right)+C\left(x_{s_{j}: t} ; \hat{\mu}, \sigma_{N}\right)- \\
& C\left(x_{t-A(n): m-1} ; \mu_{0}, \sigma_{0}\right)-C\left(x_{m: t} ; \hat{\mu}, \sigma_{N}\right)<\alpha \log (n)^{1+\delta}
\end{aligned}
$$

We can establish the probability of this using the following bound (Proposition 4 in Fisch et al. (2018)):

Lemma 3. Let $x_{1: n}$ be piecewise-Gaussian data. Choose any subset $x_{i: j}, 1 \leq i<j \leq n$, with a true changepoint at $s$, i.e., we have $x_{t} \sim \mathscr{N}\left(\mu_{1}, \sigma_{1}\right)$ for $t \in[i ; s-1]$, and $x_{t} \sim \mathscr{N}\left(\mu_{2}, \sigma_{2}\right)$ for $t \in[s ; j]$. Then, for any candidate changepoint $\tau$ and any $\epsilon>0$, there exist constants $B, n_{0}, K_{1}$ such that:

$$
C\left(x_{i: s-1} ; \mu_{1}, \sigma_{1}\right)+C\left(x_{s: j} ; \mu_{2}, \sigma_{2}\right)-C\left(x_{i: \tau-1} ; \hat{\mu}, \hat{\sigma}\right)-C\left(x_{\tau: j} ; \hat{\mu}, \hat{\sigma}\right) \leq K_{1} \log (n)
$$

is true for all $i, j$ with $P \geq 1-B n^{-\epsilon}$ when $n>n_{0}$.

Now take $i=s_{j}-A(n)+1, j=s_{j}+A(n)-1$. Note that there is one and only one changepoint within $x_{i: j}$ because of the required distance between changepoints. Applying Lemma 3 to such $x_{i: j}$ states that, conditional on an event with probability $P \geq 1-B n^{-\epsilon}$, the following is true for all $t \in\left[s_{j}, s_{j}+A(n)\right)$ :

$$
\begin{aligned}
& C\left(x_{t-A(n): s_{j}-1} ; \mu_{0}, \sigma_{0}\right)+C\left(x_{s_{j}: t} ; \hat{\mu}, \sigma_{N}\right)-C\left(x_{t-A(n): m-1} ; \mu_{0}, \sigma_{0}\right)-C\left(x_{m: t} ; \hat{\mu}, \sigma_{N}\right) \\
& \leq C\left(x_{t-A(n): s_{j}-1} ; \mu_{0}, \sigma_{0}\right)+C\left(x_{s_{j}: t} ; \mu_{N}, \sigma_{N}\right)-C\left(x_{t-A(n): m-1} ; \hat{\mu}, \hat{\sigma}\right)-C\left(x_{m: t} ; \hat{\mu}, \hat{\sigma}\right) \\
& \leq K_{1} \log (n)<\alpha \log (n)^{1+\delta}
\end{aligned}
$$

where we also used the fact that $\hat{\mu}, \hat{\sigma}=\operatorname{argmin}_{\mu, \sigma} C(x ; \mu, \sigma)$.

Therefore, with the same probability, $s_{j} \notin \bigcup_{t=s_{j}}^{s_{j}+A(n)-1} \boldsymbol{k}_{p r, t}$. Also, $s_{j} \notin \bigcup_{t=s_{j}+A(n)}^{n} \boldsymbol{k}_{p r, t}$ because then $s_{j} \leq t-A(n)$ and is not considered in the pruning scheme, and clearly $s_{j} \notin \bigcup_{t=1}^{s_{j}-1} \boldsymbol{k}_{p r, t}$. The case for $e_{j}$ follows by symmetry, and obviously no true changepoint can be pruned out if $s_{j}, e_{j}=\emptyset$, so the overall probability of retaining a true changepoint remains at $P \geq 1-B n^{-\epsilon}$.

\section{E Details of simulations and experiments}

\section{Algorithm 1 estimates the background level consistently}

In the first experiment we tested the performance of the algorithm with a fixed background parameter. Datasets were generated under three different scenarios: 
One segment. Gaussian with one signal segment: $n$ data points were drawn as $x_{t} \sim \mathcal{N}\left(\theta_{t}, 1\right)$, with $\theta_{t}=3$ at times $t \in(0.3 n ; 0.5 n]$, and 0 otherwise (background level).

Multiple. Gaussian with multiple signal segments: $n$ data points were drawn as $x_{t} \sim \mathscr{N}\left(\theta_{t}, 1\right)$, with:

$$
\theta_{t}=\left\{\begin{array}{l}
-1 \text { when } t \in(0.2 n ; 0.3 n] \cup(0.7 n ; 0.8 n] \\
1 \text { when } t \in(0.5 n ; 0.6 n] \\
0 \text { otherwise }
\end{array}\right.
$$

Heavy tail. Heavy tailed data with one signal segment: $n$ data points were drawn from the generalized $t$ distribution as $x_{t} \sim T(3)+\theta_{t}$, with $\theta_{t}=2$ at times $t \in(0.2 n ; 0.6 n]$ and 0 otherwise.

We generated time series for each of the three scenarios with values of $n$ between 30 and 750, with 500 replications for each $n$. Note that the segment positions remain the same for all sample sizes, so the increase in $n$ could be interpreted as denser sampling of the underlying function.

Each time series was analysed using Algorithm 1 to estimate $\theta_{0}$. The maximum segment length was set to $l=0.5 n$ and the penalty to $\beta=3 \log (n)^{1.1}$. The cost was computed using the Gaussian log-likelihood function with known variance. This means that the cost function was mis-specified in Scenario 3, and so provides a test of the robustness of the algorithm (although the variance was set to 3 , as expected for $T(3))$.

For comparison, in each replication we also retrieved the median of the entire time series $x_{1: n}$, which is used as the estimate of $\theta_{0}$ by Fisch et al. (2018). We also computed the quantiles of $\mathscr{N}\left(0, \sigma^{2} / \sqrt{n_{B}}\right)$ as the oracle efficiency limit based on the CLT, with $n_{B}$ the total number of background points: $n_{B}=0.8 n$ for one segment, $0.7 n$ for multiple, and $0.6 n$ for the heavy tail scenarios.

\section{Segment positions are accurately estimated by Algorithm 1}

The same simulation setup was used to evaluate the consistency of estimated segment number and positions. Data were generated and analysed with Algorithm 1 as in Section E, but we also retrieved the changepoint positions that were estimated in step 12. This corresponds to the online usage of the algorithm. We extracted the mean number of segments reported by the algorithm and also calculated the true positive rate (TPR) as the fraction of simulations in which the algorithm reported 
at least 1 changepoint within $0.05 n$ points of each true changepoint.

In each iteration, we also calculated the cost $F(n)$ (i.e. log-likelihood of a Gaussian changepoint model) to evaluate if the algorithm achieves the stated optimization objective. The cost was calculated using the segmentation and background mean estimated by the algorithm, or using the true positions of segments, and a penalty $\beta=3 \log (n)^{1.1}$ for each segment was added. The minimum reached by Algorithm 1 was as good as, or even smaller than, the cost based on true segmentation (Supplementary Table S4 in Supplementary Material F). Very little differences between the full and online versions of the algorithm were seen.

\section{Algorithm 2 recovers true segments under interference}

We generated time series under three different scenarios. In each, points $x_{1: n}$ were drawn from a Gaussian distribution with changes in mean. Series were generated for $n$ between 30 and 220, in 500 replications at each $n$.

Scenario 1. A signal segment overlapping a nuisance segment: $x_{t} \sim \mathcal{N}\left(\theta_{t}^{S}+\right.$ $\left.\theta_{t}^{N}, 1\right)$, with $\theta_{t}^{S}=2$ when $t \in(0.3 n ; 0.5 n], 0$ otherwise, and $\theta_{t}^{N}=2$ when $t \in$ $(0.2 n ; 0.7 n], 0$ otherwise.

Scenario 2. A nuisance segment and two non-overlapping signal segments: $x_{t} \sim \mathcal{N}\left(\theta_{t}^{S}+\theta_{t}^{N}, 1\right)$, with:

$$
\begin{aligned}
& \theta_{t}^{N}=1.5 \text { when } t \in(0.2 n ; 0.4 n], 0 \text { otherwise } \\
& \qquad \theta_{t}^{S}=\left\{\begin{array}{l}
3 \text { when } t \in(0.5 n ; 0.6 n] \\
-3 \text { when } t \in(0.7 n ; 0.8 n] \\
0 \text { otherwise }
\end{array}\right.
\end{aligned}
$$

Scenario 3. Many weak signal segments: $x_{t} \sim \mathcal{N}\left(\theta_{t}^{S}, 1\right), \theta_{t}^{S}=\theta_{j}$ when $t / n \in$ $(0.1 j ; 0.1 j+0.05]$ for $j=1, \ldots, 9 ; 0$ otherwise. Segment means are random, $\theta_{j} \sim$ Unif(-4, 4).

Each series was analysed by five methods. Algorithm 2 (proposed) was run with penalties $\beta=\beta^{\prime}=3 \log (n)^{1.1}$ as before. For classical detection of epidemic changes in mean, we used the R package anomaly (Fisch et al., 2018); the implementation allows separating segments of length 1 , but we treated them as standard segments and set all penalties to $3 \log (n)^{1.1}$. In both methods, background parameters were set to $\mu_{0}=0, \sigma_{0}=1$. We implemented the profile version of aPELT (Zhao and Yau, 2019) by adding an optimisation loop over the possible $\mu_{0}$ values to a PELT-style detector. The original paper also allows for series starting in a segment, which we 
did not implement as it is not the case in our simulations. The same penalty was used as before. The sparse method is a detector designed for sparse segments with known maximum length (Jeng et al., 2010). It performs a greedy search over all possible intervals up to length $l$. Background parameters are required, which we set to $\mu_{0}=0, \sigma=1$, and the threshold for segment acceptance was set to $\sqrt{2 \log (n l)}$, as in the original paper. All of the above methods allow a maximum segment length, which we set to $l=0.33 n$ in scenario $1,0.15 n$ in scenario $2,0.2 n$ in scenario 3 .

As an example of a different approach, we included the narrowest-over-threshold detector implemented in $\mathrm{R}$ package not, with default parameters. This is a nonepidemic changepoint detector that was shown to outperform most comparable methods (Baranowski et al., 2019). Since it does not include the backgroundsignal distinction, we define signal segments as regions between two successive changepoints where the mean exceeds $\mu_{0} \pm \sigma_{0}$.

As before, evaluation metrics were the mean number of segments and the TPR. For the proposed method, we required accurate segment type detection as well, i.e., a true positive is counted when the detector reported a signal start/end within $0.05 n$ of each signal changepoint. In scenario 1 , we also extracted the estimate of $\theta$ corresponding to the detected signal segment (or segment closest to $(0.3 n ; 0.5 n$ ] if multiple were detected). The average of these over the 500 replications is reported as $\hat{\theta}^{S}$.

\section{ChIP-seq data analysis}

Two ChIP-seq datasets of histone modifications in human immune cells were used in this section.

Broad Institute H3K27ac data was obtained from UCSC Genome Browser, GEO accession GSM733771, as mean read coverage in non-overlapping windows of 25 bp. We also retrieved an input control track for the same cell line from UCSC (GEO accession GSM733742). We analysed a window near the centromere of chromosome 1 , between $120,100,000$ to $120,700,000 \mathrm{bp}$. To improve runtime, data was downsampled to approximately 1000 points, each corresponding to mean coverage in a window of $500 \mathrm{bp}$.

UCI/McGill dataset was obtained from https://archive.ics.uci.edu/ml/ datasets/chipseq. Mean read coverage at $1 \mathrm{bp}$ resolution is provided, as well as peak annotations based on visual inspection. From this data, we used the H3K36me3 modification in monocyte sample ID McGill0104, AM annotation. Around the labels $L=\left\{\left(s_{i}, e_{i}\right)\right\}$ in each chromosome, we extracted read coverage for the window between $s-(e-s)$ and $e+(e-s) \mathrm{bp}, s=\min s_{i}, e=\max e_{i}$, and downsampled to 
about 1000 points as before. Based on visual inspection of the coverage, we chose a group of labels in chromosome 12, which provides a variety of annotations and a structure that appears to contain both nuisance shifts and signal peaks.

The datasets were analysed by the method proposed here (Algorithm 2), an epidemic detector from package anomaly, and the non-epidemic detector not. The length of the signal segments was limited to $50 \mathrm{kbp}$ in anomaly and the proposed method. As estimates of global $\mu_{0}$ and $\sigma_{0}$, the median and standard deviation of the unsegmented data were used. As before, penalties were set to $3 \log (n)^{1.1}$. Only segments with estimated $\theta>\mu_{0}$ are shown, as we are a priori interested in regions of increased binding.

We also used GFPOP, implemented in R package PeakSegDisk (Hocking et al., 2018): this detector has been developed specifically for ChIP-seq data processing, and models a change in Poisson rate parameter. It is intended as a supervised method, with the penalty value $\lambda$ chosen based on the best segmentation provided by the training data. Therefore, for the Broad Institute dataset, we repeated the segmentation with $\lambda \in\left\{10^{1}, 10^{2}, 10^{3}, 10^{4}, 10^{5}, 10^{6}\right\}$, and show the $\lambda$ that produces between 2 and 10 segments.

To evaluate the results quantitatively, we calculated the SIC based on each method's segmentation. We used Gaussian likelihood with parameters matching the detection status (i.e., estimated mean for the points in each segment, and $\hat{\mu}_{0}$ for points outside segments). The number of parameters was set to $3 k+2$ : a mean and 2 endpoints for each of the $k$ segments reported, and 2 for the background parameters.

\section{European mortality data analysis}

We analysed Eurostat data of weekly deaths in Spain over a three year period between 2017 and 2020. Data was retrieved from https://ec.europa. eu Data Explorer. We use the 60-64 years age group in which these trends are visually clear and thus provide a ground truth.

Four methods were used to analyse this data. For the proposed and anomaly methods, we used the median and standard deviation of the first 52 weeks of the dataset as estimates of $\mu_{0}$ and $\sigma_{0}$, respectively. Penalties in both were set to $3 \log (n)^{1.1}$, as previously. The maximum length of signal segments was set to 10 weeks, to separate seasonal effects. In addition, not was used with default parameters, defining signal as regions where the mean exceeds $\mu_{0} \pm \sigma_{0}$, and PeakSegDisk with a basic grid search to select the penalty as before. 


\section{F Supplementary tables}

Table S3: Consistency of the number and position of estimated changepoints. Time series simulated in three different scenarios were analysed using Algorithm 1, in 500 replications for each $n$. The mean number of reported segments and the TPR (fraction of replications when a changepoint was detected within $0.05 n$ of each true changepoint) are shown. The number of true segments was 1,3 , and 1 for scenarios one segment, multiple, heavy tail respectively. The same time series were also analysed in an online manner, i.e., without Step 13 of the algorithm; the results are shown on the right of the table.

\begin{tabular}{lcrrrr}
\hline & \multicolumn{3}{c}{ (Full algorithm) } & \multicolumn{2}{c}{ (without step 13) } \\
Scenario & $n$ & Mean \# segm. & TPR & Mean \# segm. & TPR \\
\hline one segm. & 30 & 1.112 & 0.932 & 1.138 & 0.924 \\
& 90 & 1.040 & 0.998 & 1.056 & 0.998 \\
& 180 & 1.054 & 1.000 & 1.060 & 1.000 \\
& 440 & 1.032 & 1.000 & 1.040 & 1.000 \\
& 750 & 1.028 & 1.000 & 1.034 & 1.000 \\
\hline multiple & 30 & 0.552 & 0.000 & 0.584 & 0.000 \\
& 90 & 1.096 & 0.008 & 1.050 & 0.004 \\
& 180 & 1.762 & 0.086 & 1.720 & 0.080 \\
& 440 & 2.900 & 0.824 & 2.854 & 0.782 \\
& 750 & 3.010 & 0.992 & 3.008 & 0.988 \\
\hline heavy tail & 30 & 0.644 & 0.142 & 0.662 & 0.132 \\
& 90 & 1.426 & 0.592 & 1.416 & 0.570 \\
& 180 & 1.930 & 0.878 & 1.932 & 0.866 \\
& 440 & 2.798 & 0.998 & 2.798 & 0.996 \\
& 750 & 3.692 & 1.000 & 3.664 & 1.000 \\
\hline
\end{tabular}


Table S4: Final cost corresponding to segmentations obtained by Algorithm 1 (either full or excluding step 13), or the true segmentation. Cost is the log-likelihood of a Gaussian epidemic changepoint model with the corresponding segmentation. Given numbers are mean \pm SD over 500 iterations for each setting.

\begin{tabular}{lc|r|r|r}
\hline Scenario & $\mathrm{n}$ & Full algorithm & Without step 13 & True \\
\hline one segm. & 30 & $53.4 \pm 5.4$ & $53.6 \pm 5.6$ & $52.9 \pm 3.9$ \\
& 90 & $142.0 \pm 7.9$ & $142.1 \pm 8.0$ & $141.7 \pm 6.7$ \\
& 180 & $270.9 \pm 10.3$ & $270.9 \pm 10.3$ & $270.8 \pm 9.7$ \\
& 440 & $642.9 \pm 15.1$ & $643.1 \pm 15.2$ & $643.1 \pm 14.8$ \\
& 750 & $1084.7 \pm 19.0$ & $1084.7 \pm 19.0$ & $1084.9 \pm 18.8$ \\
\hline multiple & 30 & $48.5 \pm 7.3$ & $48.6 \pm 7.4$ & $73.9 \pm 3.5$ \\
& 90 & $145.3 \pm 10.2$ & $145.1 \pm 10.2$ & $170.3 \pm 6.6$ \\
& 180 & $286.2 \pm 12.9$ & $286.0 \pm 12.7$ & $304.9 \pm 9.5$ \\
& 440 & $676.5 \pm 14.6$ & $676.1 \pm 14.9$ & $682.7 \pm 14.2$ \\
& 750 & $1121.4 \pm 19.9$ & $1121.4 \pm 19.9$ & $1126.9 \pm 19.9$ \\
\hline heavy tail & 30 & $63.1 \pm 8.4$ & $63.2 \pm 8.4$ & $68.5 \pm 13.5$ \\
& 90 & $185.2 \pm 14.1$ & $185.1 \pm 14.1$ & $191.4 \pm 34.9$ \\
& 180 & $361.1 \pm 19.8$ & $361.4 \pm 20.0$ & $367.6 \pm 40.6$ \\
& 440 & $866.4 \pm 37.2$ & $866.6 \pm 37.1$ & $906.7 \pm 385.3$ \\
& 750 & $1468.1 \pm 51.3$ & $1468.4 \pm 51.4$ & $1507.7 \pm 303.1$ \\
\hline
\end{tabular}

Table S5: Comparison of Algorithm 2 detections without pruning (Full) or with global pruning (Pruned). Of the 7500 simulation runs, all runs where the two options produced any difference in segments were identified. All detections from these runs are shown here. Segment types are $\mathrm{N}$ - nuisance, $\mathrm{S}$ - signal.

\begin{tabular}{|c|c|c|c|c|c|c|c|c|}
\hline \multirow[b]{2}{*}{ Scenario } & \multirow[b]{2}{*}{$n$} & \multirow[b]{2}{*}{ Run number } & \multicolumn{3}{|c|}{ (Full) } & \multicolumn{3}{|c|}{ (Pruned) } \\
\hline & & & Segm. type & Start & End & Segm. type & Start & End \\
\hline \multirow[t]{9}{*}{2} & 30 & 28 & $\mathrm{~N}$ & 7 & 27 & $\mathrm{~S}$ & 7 & 9 \\
\hline & 30 & 28 & S & 14 & 15 & $S$ & 16 & 18 \\
\hline & 30 & 28 & S & 21 & 24 & $S$ & 21 & 24 \\
\hline & 30 & 268 & $\mathrm{~N}$ & 7 & 25 & $\mathrm{~N}$ & 7 & 12 \\
\hline & 30 & 268 & S & 13 & 15 & S & 16 & 18 \\
\hline & 30 & 268 & S & 21 & 24 & $S$ & 21 & 24 \\
\hline & 60 & 252 & $S$ & 31 & 36 & $S$ & 31 & 36 \\
\hline & 60 & 252 & $\mathrm{~N}$ & 43 & 59 & $S$ & 43 & 48 \\
\hline & 60 & 252 & S & 49 & 56 & $S$ & 58 & 59 \\
\hline
\end{tabular}




\section{Bibliography}

Baranowski, R., Chen, Y. and Fryzlewicz, P. (2019) Narrowest-over-threshold detection of multiple change points and change-point-like features. Journal of the Royal Statistical Society: Series B (Statistical Methodology), 81, 649-672.

Bottou, L. (1998) Online algorithms and stochastic approximations. In Online Learning and Neural Networks (ed. D. Saad). Cambridge, UK: Cambridge University Press.

Fisch, A. T. M., Eckley, I. A. and Fearnhead, P. (2018) A linear time method for the detection of point and collective anomalies. arXiv preprint: 1806.01947.

Harvey, N. J. A., Liaw, C., Plan, Y. and Randhawa, S. (2019) Tight analyses for non-smooth stochastic gradient descent. In Proceedings of the Thirty-Second Conference on Learning Theory (eds. A. Beygelzimer and D. Hsu), vol. 99 of Proceedings of Machine Learning Research, 1579-1613. Phoenix, USA: PMLR.

Hocking, T. D., Rigaill, G., Fearnhead, P. and Bourque, G. (2018) Generalized functional pruning optimal partitioning (GFPOP) for constrained changepoint detection in genomic data. arXiv preprint: 1810.00117.

Jeng, X. J., Cai, T. T. and Li, H. (2010) Optimal sparse segment identification with application in copy number variation analysis. Journal of the American Statistical Association, 105, 1156-1166.

Killick, R., Fearnhead, P. and Eckley, I. A. (2012) Optimal detection of changepoints with a linear computational cost. Journal of the American Statistical Association, 107, 15901598.

Purkayastha, S. (1998) Simple proofs of two results on convolutions of unimodal distributions. Statistics \& Probability Letters, 39, 97-100.

Zhao, Z. and Yau, C. Y. (2019) Alternating pruned dynamic programming for multiple epidemic change-point estimation. arXiv preprint: 1907.06810.

Zidek, J. V. and van Eeden, C. (2003) Uncertainty, entropy, variance and the effect of partial information. In Mathematical statistics and applications: Festschrift for Constance van Eeden (eds. M. Moore, S. Froda and C. Léger), vol. 42 of Lecture Notes-Monograph Series, 155-167. Institute of Mathematical Statistics. 


\title{
A changepoint prefilter for sound event detection in long-term bioacoustic recordings
}

\author{
Julius Juodakis ${ }^{1}$, Stephen Marsland, Nirosha Priyadarshani
}

Published in The Journal of the Acoustical Society of America, 150, 2469-2478 (2021), reproduced here with minor revisions

\begin{abstract}
Long-term soundscape recordings are useful for a variety of applications, most notably in bioacoustics. However, the processing of such data is currently limited by the ability to efficiently and reliably detect target sounds, which are often sparse and overshadowed by environmental noise. This paper proposes a sound detector based on changepoint theory applied to a wavelet representation of the sound. In contrast to existing methods, in this framework theoretical analysis of the detector's performance and optimality for downstream applications can be made. The relevant statistical and algorithmic developments to support these claims are presented. The method is then tested on a real task of detecting two bird species in acoustic surveys. Compared to commonly-used alternatives, the proposed method consistently produced a lower false alarm rate and improved survey efficiency, as measured by the precision of the inferred population size. Finally, we demonstrate how the method can be combined with a simple classifier to detect cat sounds in domestic recordings, an example from DCASE 2018. The resulting performance is comparable to state-of-the-art deep learning models, while requiring much less

\footnotetext{
${ }^{1}$ Corresponding author. Email: julius. juodakis@sms .vuw. ac.nz. J. Juodakis, S. Marsland and N. Priyadarshani at School of Mathematics and Statistics, Victoria University of Wellington, New Zealand.
} 
training data.

\section{Introduction}

Over the last decade, long-term sound recordings have emerged as an inexpensive way to monitor various urban and natural environments. In bioacoustics, autonomous recorders are already routinely used for monitoring populations of endangered species (e.g. Loeb et al., 2015; Robertson and Colbourne, 2017; Wright et al., 2018), and more broadly in researching animal behaviour (Gibb et al., 2018). Many more applications are being explored, for example, elephant warning systems (Zeppelzauer et al., 2015), digital beekeeping (Zgank, 2019), and a range of ideas for assisted living (Droghini et al., 2017; Castillo-Escario et al., 2019).

A major bottleneck for implementing such sensors in practice is the ability to identify target events in the vast amounts of data produced. Standard tools for automated sound analysis perform poorly on long-term recordings. This difference was observed directly in the BirdCLEF challenge (Goëau et al., 2018): in the task of identifying bird species from targeted clips, the best solutions showed 70-80\% mean average precision, but on long-term data this plummeted to below $20 \%$ for the same algorithms. This variation is because the target events in real problems are usually sparse, distant, and overshadowed by dynamic environmental noise (Barker et al., 2018; Priyadarshani et al., 2018). The noise not only degrades the target sounds, but also creates false positive detections when analysing such data. Developing noise-robust analysis tools could greatly improve the effectiveness of acoustic monitoring.

Modern sound event detectors typically include a classifier that views short windows of sound and classifies each as target/non-target (monophonic detection) or lists all events present (polyphonic). Most commonly, convolutional neural networks (CNNs) viewing spectrograms are used, but other approaches are also possible (Hayashi et al., 2018). However, for most practical purposes, such as inference based on event counts or extraction of further features of the events, the window labels need to be combined into segments, each representing a single event. This can be done by post-processing, or by a prefilter (also known as an activity detector). Pre-filtering algorithms use simple rules to define segment boundaries before classification, thus reducing the computational load and also making the latter task easier (Phan et al., 2017). In small-data situations where CNNs are not available, particularly in bioacoustics, prefilters are combined with simple classifiers or used 
on their own, followed by human review of the segments (Priyadarshani et al., 2018).

Both post-processing and prefiltering workflows are currently chosen ad hoc and rarely compared. Post-processing typically consists of various smoothing and trimming steps (Serizel et al., 2018; Hayashi et al., 2018). Pre-filtering algorithms start by detecting energy peaks in time domain (Ramli and Jaafar, 2016; Liu et al., 2018) or high-energy bins in spectrogram or wavelet representation (Lasseck, 2013; Priyadarshani et al., 2020). These are then combined by smoothing, joining gaps, and trimming. To combine spectrogram peaks, morphological image processing operations are commonly used (Lasseck, 2016; Wang et al., 2021). Composition of these steps can give rise to complex procedures, such as the manatee detector from Merchan et al. (2019), which uses RMS of the autocorrelation of a wavelet transform, which is then smoothed, peaks selected, trimmed to relevant time and frequency, and interpolated to produce segments. Alternatively, neural networks can be trained to specifically learn event boundaries (Pankajakshan et al., 2019), providing that one has large amounts of data. Overall, the empirical, theoretical and computational differences between these methods are mostly unknown, so it is not clear if they are optimal, and how they generalize to different sounds and environments. Improvements here may not only increase detection performance, but also ensure accurate inference and more efficient downstream processing.

In this paper, we propose a prefiltering algorithm for monophonic detection of sound events, based on changepoint theory. This framework provides a strong statistical basis, which enables guarantees about detector performance and failure modes, as well as computational efficiency (Niu et al., 2016; Truong et al., 2020). Further, to impart some noise robustness, our method uses segment duration to separate transient target events from slow-changing noise. We have previously shown that this principle can be used to robustly detect changes in the mean of a time series (Juodakis and Marsland, 2020). We reason that it can also be applied to sound, because some knowledge of the expected duration is often available or easy to learn, and can be informative even when the signal and noise are similar in nature, such as for detecting vocal activity over babble noise (Barker et al., 2018).

Specifically, we extend the method from Juodakis and Marsland (2020) to detect changes in the energy of a wavelet coefficient series. We provide proofs that this approach consistently detects the number and position of events under Gaussian noise, and discuss its robustness to parameter values. We then compare it experimentally with other detectors in two tasks. In bird surveys, the proposed method is 
applied as a stand-alone detector, and provides the best event detection ( $F_{1}$ score) and highest overall monitoring efficiency compared to commonly-used methods. For a task of detecting cat sounds (DCASE 2018 Task 4 data), the method is used as a prefilter for a simple classifier, and comparable performance to state-of-the-art CNN methods is achieved, while using much less training data.

\section{Detection theory and new developments}

\subsection{Changepoint detection framework}

Consider a time series $x_{0: n}$ generated by a parametric distribution $f$ that changes at points $t_{1}, \ldots, t_{m}$. Let $\theta_{i}$ be the value of the changing parameter, attained at $t_{i}$. We will define a piecewise model using set notation, so e.g. the points $\left[t_{i}, t_{i+1}\right)$ comprise a segment, and $\bigcup_{i=1}^{m}\left[t_{i}, t_{i+1}\right)$ are all the points in $m$ segments. Then the probability density function corresponding to this classical changepoint model is:

$$
p\left(x_{j}\right)=\sum_{i=0}^{m} f\left(x_{j} \mid \theta_{i}\right) \mathbb{1}\left(j \in\left[t_{i}, t_{i+1}\right)\right) .
$$

We adopt the convention $t_{0}=0, t_{m+1}=n$ to simplify notation when useful, and use $\mathbb{1}$ to denote the indicator function.

A variation of this framework is the so-called epidemic changepoint model. Segments are defined by pairs of changepoints $T=\left(s_{1}, e_{1}\right), \ldots,\left(s_{m}, e_{m}\right)$. Outside segments the data follows a shared background model. The overall data distribution then is:

$$
p\left(x_{j}\right)=\sum_{i=1}^{m} f\left(x_{j} \mid \theta_{i}\right) \mathbb{1}\left(j \in\left[s_{i} ; e_{i}\right]\right)+f\left(x_{j} \mid \theta_{0}\right) \mathbb{1}\left(j \notin \bigcup_{i=1}^{m}\left[s_{i}, e_{i}\right]\right) .
$$

The background parameter $\theta_{0}$ may be known a priori or estimated from the data.

When the data is an appropriately chosen representation of sound, the $(s, e)$ pairs correspond to onset and offset times of acoustic events. Specifically, we expect sound events to appear as periods of larger energy, i.e., higher variance, of $x_{j}$. We will show in Section 2.5 that this is the case when $x_{j}$ is obtained by wavelet transform. This makes it possible to use the extensive literature on changepoint theory (reviewed in Aminikhanghahi and Cook (2016); Niu et al. (2016); Truong et al. (2020)) to guide our design and analysis of sound event detectors. 


\subsection{Estimation of changepoints}

Our goal is to obtain consistent estimates of positions $\hat{s}_{i}, \hat{e}_{i}$, as well as an accurate estimate of the number of events $\hat{m}$, in the sense that $\left|\hat{s}_{i}-s_{i}\right| / n \rightarrow 0,\left|\hat{e}_{i}-e_{i}\right| / n \rightarrow$ $0,|\hat{m}-m| \rightarrow 0$ as the number of data points $n$ increases. It has been shown that when $f$ is Gaussian such consistency is achieved by estimators based on $L_{0}$-penalized likelihood (Yao and Au, 1989; Zheng et al., 2019; Fisch et al., 2018). Define a segment $\operatorname{cost} C\left(x_{a: b} ; \theta\right)=-2 \sum_{i=a}^{b} \log f\left(x_{i} \mid \theta\right)$. Then the cost of a proposed segment set $\hat{T}=\left(\hat{s}_{1}, \hat{e}_{1}\right), \ldots,\left(\hat{s}_{\hat{m}}, \hat{e}_{\hat{m}}\right)$ is:

$$
\hat{C}\left(x_{0: n}, \hat{T}, \beta\right)=\sum_{i=1}^{\hat{m}} \min _{\theta} C\left(x_{\hat{s}_{i}: \hat{e}_{i}} ; \theta\right)+\sum_{j \notin \cup[\hat{s}, \hat{e}]} C\left(x_{j} ; \theta_{0}\right)+\hat{m} \beta .
$$

This is exactly the likelihood of model (2) with an added penalty term $\hat{m} \beta$ that controls the number of changepoints. The desired estimates are obtained by minimising this cost over $\hat{T}$, with a user-chosen $\beta$.

Finding efficient ways to minimise $\hat{C}$ is a major challenge, as directly testing all possible $\hat{T}$ s is computationally infeasible. A variety of algorithms have been proposed, differing in computational complexity, guarantees of finding the correct solution, and assumptions made about the signal process. Given known background parameter $\theta_{0}$, exact solutions can be obtained efficiently using dynamic programming and pruning (Killick et al., 2012). When $\theta_{0}$ is to be estimated, exact solutions may still be achieved, but at the cost of intensive computations (Zhao and Yau, 2021) or assuming small fraction of non-background points (Fisch et al., 2018). Alternatively, approximate solvers can be used, such as WBS by Fryzlewicz (2014). In Juodakis and Marsland (2020) we proposed a fast algorithm based on stochastic gradient descent that can estimate epidemic changes in mean when $f(\cdot \mid \theta)$ is a symmetric distribution (Algorithm 1). It uses dynamic programming to minimise $F(n)$, which approximates $\hat{C}\left(x_{0: n}, \hat{T}, \beta\right)$.

To apply this algorithm for changes in variance, the main challenge is to show that the estimates of background variance produced by it converge to $\theta_{0}$, as in Theorem 1.

Theorem 1. Consider the problem of epidemic changes in variance, i.e., data $x_{0: n}$ generated from model (2), with $f(x \mid \theta)=1 / \sqrt{2 \pi \theta^{2}} \exp \left(-\frac{x^{2}}{2 \theta^{2}}\right)$. The background variance $\theta_{0}^{2}$ is unknown. Denote by $w_{t}$ the estimate of $\theta_{0}$ obtained by analysing $x_{0: t}$ using Algorithm 1. The sequence $\left\{w_{t}\right\}$ converges to $\left(\theta_{0}-\epsilon, \theta_{0}+\epsilon\right)$ almost surely, with $\epsilon \rightarrow 0$ as the number of background points between successive segments $n \rightarrow \infty$. 


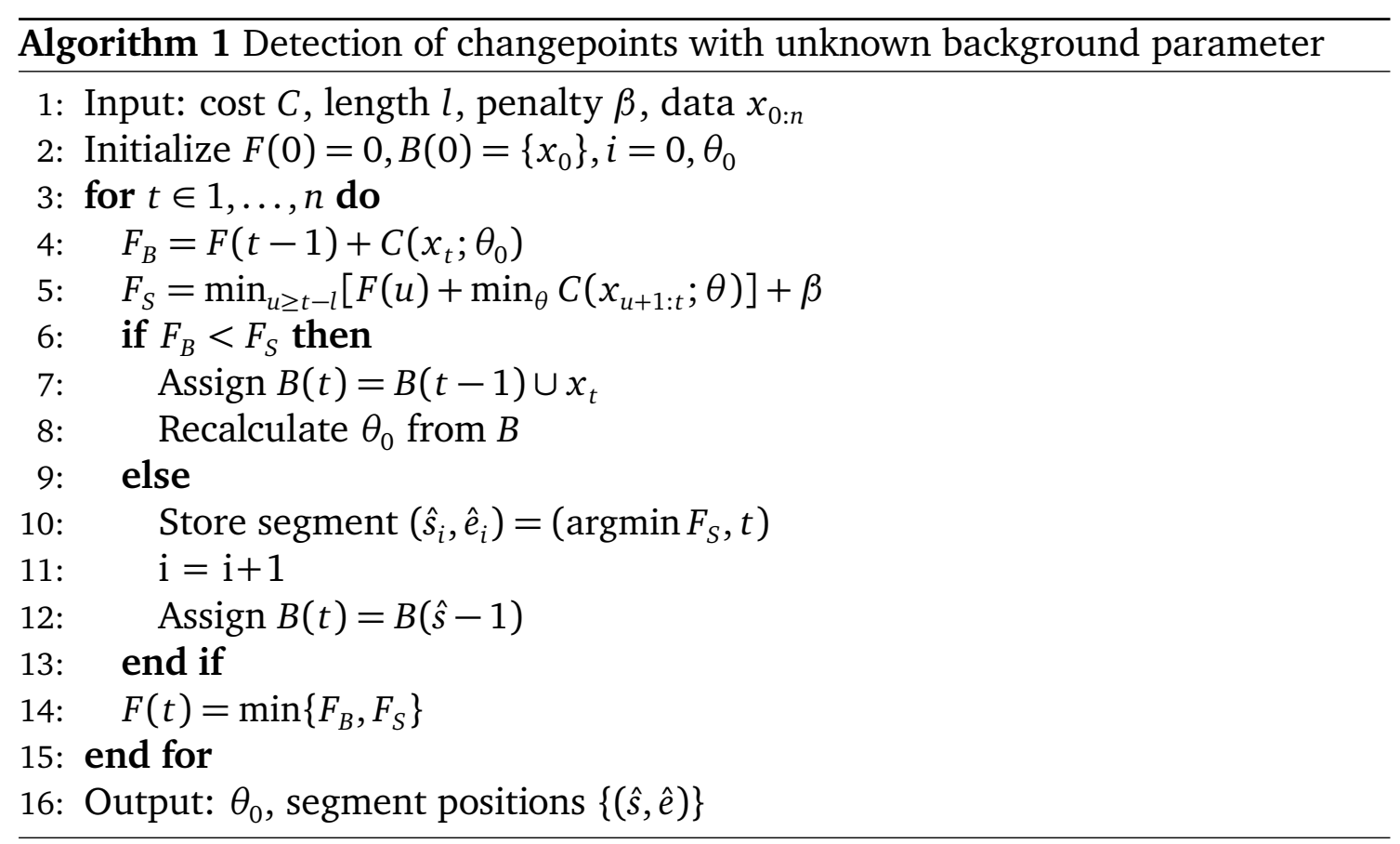

The proof is given in Supplementary Material A. It is based on convergence results for stochastic gradient descent-like algorithms. As shown in Juodakis and Marsland (2020), given convergence, algorithms of this type will estimate epidemic changepoints consistently.

\subsection{Detection in the presence of nuisance changes}

The changepoint models introduced so far assume a stable background level. To model a dynamic background, in Juodakis and Marsland (2020) we introduced nuisance states: these are represented by segments $\left(s_{i}^{N}, e_{i}^{N}\right), i=1, \ldots, k$, in which the background parameter shifts to $\theta_{i}^{N}$. Target segments $(s, e)$ may overlap with the background or these nuisance states, leading to the following model:

$$
\begin{aligned}
\theta^{S} & =\sum_{i=1}^{m} \mathbb{1}\left(j \in\left[s_{i} ; e_{i}\right]\right) \theta_{i}, \\
\theta^{N} & =\sum_{i=1}^{k} \mathbb{1}\left(j \in\left[s_{i}^{N} ; e_{i}^{N}\right]\right) \theta_{i}^{N}+\mathbb{1}\left(j \notin \cup\left[s^{N}, e^{N}\right]\right) \theta^{0}, \\
p\left(x_{j}\right) & =f\left(x_{j} \mid h\left(\theta^{S}, \theta^{N}\right)\right) .
\end{aligned}
$$

The function $h$ determines the interaction between the overlapping processes: for example, when additivity is assumed, it may be chosen so that $f\left(\cdot \mid h\left(\theta^{S}, \theta^{N}\right)\right)=f_{X+Y}$ for $X$ and $Y$ independent, $f_{X}=f\left(\cdot \mid \theta^{S}\right), f_{Y}=f\left(\cdot \mid \theta^{N}\right)$. We assumed that in every 
case $x$ still follows a distribution from the family $f$, but even that is not necessary in general.

To ensure that this model is identifiable, it is assumed that target segments are shorter than the nuisance ones, and also that the target segments are either entirely within or totally outside a nuisance segment:

$$
\begin{gathered}
\max _{i}\left(e_{i}-s_{i}\right)<\min _{i}\left(e_{i}^{N}-s_{i}^{N}\right) \\
\forall i, j:\left[s_{i}, e_{i}\right] \cap\left(s_{j}^{N}, e_{j}^{N}\right)=\emptyset \text { or }\left[s_{i}, e_{i}\right] .
\end{gathered}
$$

When two segments overlap, we will also restrict their change directions to match:

$$
\theta^{S}>\theta^{N}>\theta^{0} \text { or } \theta^{S}<\theta^{N}<\theta^{0}
$$

While changepoints can be located without this assumption, it ensures identifiability of the segment types. This restriction is mild when $\theta$ is a scale parameter, since it follows naturally unless the noise and signal processes are negatively correlated.

Similarly to (3), we can define a penalized cost for any proposed set of target and nuisance segments $\hat{T}$. Let $X_{S}=\bigcup_{i} x_{\hat{s}_{i}: \hat{e}_{i}}$ be all target points, $X_{N}=\bigcup_{j} x_{\hat{s}_{j}^{N}: \hat{e}_{j}^{N}}$ all nuisance points. Then:

$$
\begin{aligned}
& \hat{C}^{N S}\left(x_{0: n}, \hat{T}, \beta\right)=\sum_{x_{j} \notin X_{S} \cup X_{N}} C\left(x_{j} ; \theta_{0}\right)+\min _{\theta}\left[\sum_{i=1}^{m} C\left(x_{\hat{s}_{i}: \hat{e}_{i}} \backslash X_{N} ; \theta_{i}^{S}\right)+\right. \\
& \left.+\sum_{j=1}^{k} C\left(x_{\hat{s}_{j}^{N}: \hat{e}_{j}^{N}} \backslash X_{S} ; \theta_{j}^{N}\right)+\sum_{j=1}^{k} \sum_{i=1}^{m} C\left(x_{\hat{s}_{i}: \hat{e}_{i}} \cap x_{\hat{s}_{j}^{N}: \hat{e}_{j}^{N}} ; h\left(\theta_{i}^{S}, \theta_{j}^{N}\right)\right)\right]+(k+m) \beta .
\end{aligned}
$$

This cost can be minimized using a method based on Algorithm 1. Compared to the epidemic model in (2), the main challenge is that the two kinds of segments may overlap: this creates an epidemic changepoint problem within each proposed nuisance segment. The complete process is presented as Algorithm 2 in Supplementary Material D. The segment type (nuisance or not) is determined by its length; the boundary $l$ is set by the user.

The consistency of changepoints estimated this way has not been shown previously. We present this result next. 


\subsection{Consistency results}

We focus on the case of detecting variance changes in Gaussian noise. Specifically, assume data $x_{0: n}$ is generated from the changepoint model with additive nuisances in (4), $f$ is Gaussian, and $\theta$ is its $\sigma$ parameter. Segments are estimated by minimising the cost $\hat{C}^{N S}$ with a penalty of the form $\beta=\alpha \log (n)^{1+\delta} ; \alpha, \delta>0$.

In order to meaningfully discuss the probability of detecting all events in a dataset, a minimum signal strength that is exceeded by every event must be set (see Niu et al. (2016) for analysis of such bounds in various detectors). Following Fisch et al. (2018), define the following measure of change strength at changepoint $t_{i}$ :

$$
\Delta_{i}=\log \left(\frac{\gamma_{i}}{2 \gamma_{i+1}}+\frac{\gamma_{i+1}}{2 \gamma_{i}}\right)
$$

with $\gamma_{i}=h\left(\theta_{i}^{S}, \theta_{i}^{N}\right)$ denoting the parameter corresponding to data range $t_{i-1}: t_{i}$. We require that all pairs of subsequent changepoints $t_{i}, t_{i+1}$ satisfy the following, with some $\delta_{2}>\delta$ :

$$
t_{i+1}-t_{i} \geq \frac{\log (n)^{1+\delta_{2}}}{\min \left(\Delta_{i}, \Delta_{i}^{2}, \Delta_{i+1}, \Delta_{i+1}^{2}\right)} .
$$

We prove two consistency results. For space reasons, full statements and proofs are written out in the Supplementary Material (Theorems 2 and 3), and we provide a summary of the results here:

Result 1. If the length bound $l$ is chosen accurately, so that $l-\max \left|s_{i}-e_{i}\right| \geq$ $\log (n)^{1+\delta_{2}}$ and $\min \left|s_{j}^{N}-e_{j}^{N}\right|-l \geq \log (n)^{1+\delta_{2}}$, then minimising the penalized cost $\hat{C}^{N S}$ will consistently estimate the number, type and positions of all changepoints. The error in the position estimates is bounded by $\mathscr{O}\left(\log (n)^{1+\delta}\right)$ with $P \rightarrow 1$.

Result 2. If the chosen length bound $l$ is overestimated, so $l \geq \min \left|s_{j}^{N}-e_{j}^{N}\right|-$ $\log (n)^{1+\delta_{2}}$, then minimising the penalized cost $\hat{C}^{N S}$ will consistently estimate all non-nuisance changepoints, with the same error bound.

Thus, the proposed detection method - minimising the penalized cost in (5) - produces good estimates of the target segment positions $s_{i}, e_{i}$ and their number, and is robust to nuisance changes. It requires some knowledge of maximum segment length $l$, but is not sensitive to its overestimation. The penalty parameter $\alpha$ determines the detection threshold, and can be set for a particular problem in a training stage. 


\subsection{Wavelet coefficient series}

So far, we have discussed the analysis of a hypothetical uncorrelated Gaussian process $x_{0: n}$. Natural sound recordings, however, have a strong correlation structure, and naïvely applying changepoint detection to them could be ineffective: it was shown recently that the number of events can be over-estimated by as much as 1000-fold this way (Hubert et al., 2019).

To allow changepoint analysis of sound, we transform such series into a wavelet packet tree (WPT) representation. Denote the original signal as $\left\{w_{0,0, t}\right\}_{t=0, \ldots, n}$; this is the root node of the WPT. The remaining nodes for $j=1, \ldots, J$ and $k=1, \ldots, 2^{j}$ store the sequences produced by iteratively applying a pair of orthogonal filters determined by the chosen wavelet. Thus, each node $\left\{w_{j, k, t}\right\}_{t \geq 0}$ is a band-limited and transformed component of the original signal. It can be used to reconstruct a bandpassed signal, or subjected to frequency-domain analysis directly.

Now sound events can be detected by applying changepoint detection to the wavelet coefficient series $\left\{w_{j, k, t}\right\}_{t \geq 0}$. The particular node $(j, k)$ can be chosen based on prior knowledge, or selected in a training stage. If the node corresponds to a narrow frequency band, then the resulting bandpassing can already impart some specificity to the detection. The main benefit of WPT is that it decorrelates the sound: a wide variety of noise processes, including $1 / f$ noise, will result in wavelet coefficients that are close to iid Gaussian (Wornell, 1993; Chang and Stein, 2013). Notably, the transformation with orthogonal wavelets preserves energy (Mallat, 2009), so events in the original series will also appear as changes of variance in $\left\{w_{j, k, t}\right\}_{t \geq 0}$, provided that node $(j, k)$ covers the relevant frequency band. This makes the wavelet coefficients suitable for changepoint detection.

\section{Experimental methods}

\subsection{Implementation}

The proposed changepoint prefilter algorithm was implemented in C and Python in the AviaNZ software (Marsland et al., 2019). Each analysed file is first converted to a WPT, and wavelet coefficients $w_{j, k, t}$ corresponding to a chosen node $(j, k)$ are extracted. As the main input to the detection stage (Algorithm 2), we use the RMS energy of these coefficients over non-overlapping windows of size $S$ : $x_{\tau}=\sqrt{\sum_{i=0}^{S-1} w_{j, k, \tau S+i} / S}$. This allows the user to reduce the computational time by setting $S$ to a resolution that is relevant to their analysis. It also smoothes out 
impulsive noise. Multiple nodes can be specified, in which case each is analysed individually, and any overlapping detections then merged. The detector parameters thus consist of a set of nodes $\{(j, k)\}$, window size $S$, maximum length of the event $l$, and penalty $\alpha$.

We estimate the global background $\theta_{0}$ using the 10th percentile of $x_{\tau}$, since such an estimate is robust to a large fraction of non-background sounds, or - when the fraction is small - the method can adjust to the true background by including large nuisance segments.

Two alternative prefiltering algorithms are used for comparison:

1. Wavelet filter. A wavelet energy detector previously described in Priyadarshani et al. (2020). The signal is reconstructed from selected wavelet nodes, smoothed, energy peaks detected with an adaptive threshold and postprocessed into segments. It was shown to outperform a variety of other machine learning classifiers (Priyadarshani et al., 2020).

2. Median clipping. The algorithm used in the winning solutions to previous CLEF challenges (Lasseck, 2013, 2016). It detects spectrogram peaks and converts them to segments using image processing techniques. To utilize the frequency information, we first reconstructed the signal from selected wavelet nodes, antialiased as described in Priyadarshani et al. (2020), and then created a spectrogram and analysed it with median clipping.

All three methods require selecting the wavelet nodes and a detection threshold, which controls the tradeoff between false negatives and false positives. These parameters were tuned by a grid search on a small manually-annotated training dataset. We set the main threshold in all methods to produce approximately $75 \%$ recall on this data (ratio of true positives to manually labelled positives, here measured at $1 \mathrm{~s}$ resolution). This way, we expect the methods to differ mainly in their false alarm rate. Additionally, the changepoint method needed a parameter $l$, which we estimated as the maximum call length observed in the training set. Window size $S$ was set a priori to around 1/10th of expected shortest call length for each detector.

The outputs of each method are putative call timestamps, which can then be used for further classification, human review, or as input to inference models. (The changepoint detector also reports nuisance segments which we do not analyse further.) An example illustrating these outputs and the effects of different parameters is shown in Figure 1. The sound clip and filter settings used in this example are taken from the kiwi survey described further below. 


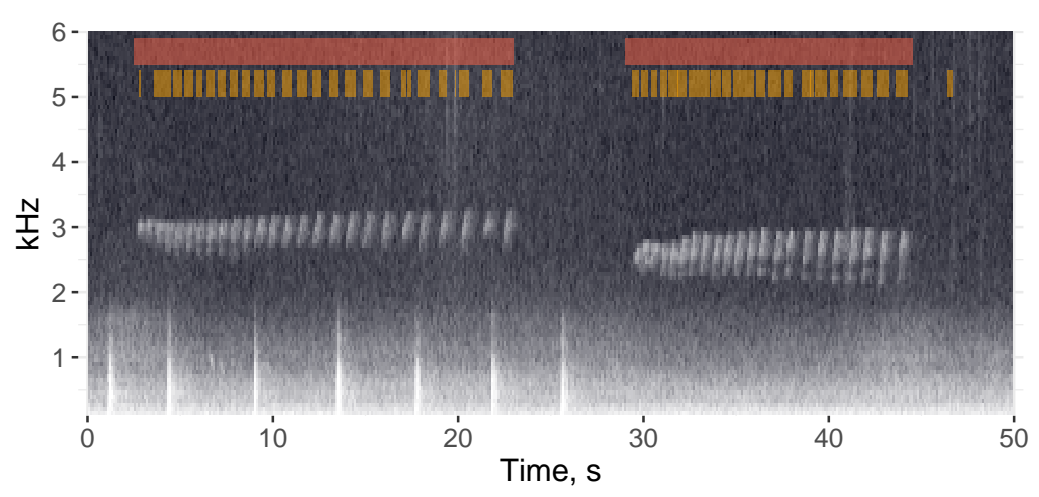

Figure 1: An example spectrogram showing two calls of little spotted kiwi from the Zealandia survey. Analysing this file with the proposed changepoint detector, trained for kiwi, produced two segments (timestamps), shown as red bars above. The same detector can be easily adapted to detect syllables instead, by reducing the maximum duration parameter $l$ (from 45 to $0.5 \mathrm{~s}$ ) and the smoothing window $S$ (from 0.5 to $0.05 \mathrm{~s}$ ). The resulting detections are shown in orange. Note the low frequency noises caused by nearby fireworks.

\subsection{Application to bioacoustic surveys}

To test the proposed algorithm in a real bioacoustics situation, we analysed two acoustic bird surveys. The methods were evaluated by reviewing the detections, fitting a population size spatial capture-recapture (SCR) model, and comparing the resulting precision of its estimates. This way, various detection errors and their downstream effects are summarised in a single value. We also present event-based $F_{1}$ scores, based on comparing the algorithm detections to manual annotations. This metric is commonly used in acoustic challenges (Serizel et al., 2018) and allows easy comparison between methods, although it does not always reflect the primary goals when the detections are used in bioacoustics (see Juodakis et al. (2021) for further discussion of these metrics).

The SCR framework is used in ecology to account for imperfect detection when observing animals (Dawson and Efford, 2009; Borchers et al., 2015). When applied to acoustic surveys, SCR consists of two components: a call emission model (usually a spatial Poisson process with intensity $D$ ), and a detection function $g(d)$, which defines the probability of detecting a call emitted at distance $d$ from the recorder. Parameters of both components are estimated from the observed call data across recorders. The density $D$ is a proxy for the true number of sound sources in the area (i.e., number of calling animals), and is therefore the main target of inference in ecology, while the detection function reflects microphone sensitivity, acoustic properties of the landscape, detection algorithm used, etc. (Stevenson et al., 2015). This separation means that, for example, if two surveys are conducted with differ- 
ent equipment, these differences will be captured in the detection parameters, and density estimates $\hat{D}$ remain comparable.

However, changes in methods will impact the precision (standard error) of $\hat{D}$. Low sensitivity, i.e., high numbers of missed calls means that fewer data points are available for estimation and thus the precision suffers. Other factors, such as higher equipment cost or false alarms produced by a detector, increase the effort or budget needed per true detection. Since the total effort of a survey is typically fixed, this reduces the sample size that can be obtained, again impacting the precision (Juodakis et al., 2021). Thus, the standard error (SE) of $\hat{D}$ summarises various differences between sound processing methods in a single value. Further discussion on this is presented in Juodakis et al. (2021).

We applied this framework to compare the detection methods for the two bird surveys. For each method, we considered an equal number of raw detections (by selecting the annotations within the first $p_{i}=n_{i} / \max n \times 100 \%$ of each 5-minute window, where $n_{i}$ is the number of detections for method $i$ ). This equalizes the review effort across all methods. Clocks were post-hoc synchronized across the recorders using the method described in Juodakis et al. (2021), and contiguous detections merged into a single call. We assume that any calls that overlap in time across multiple recorders are recaptures of a single cue.

An SCR model was then fitted to the detections, using R package ascr (Stevenson et al., 2015). We used the default half-normal detection function $g(d)=$ $g_{0} \exp \left(-d^{2} / 2 \sigma^{2}\right)$. Recorder positions were supplied in New Zealand Transverse Mercator projection (LINZ, 2008), and an integration mask was constructed as $1200 \mathrm{~m}$ discs around each recorder. The survey length was set to $p_{i}$ for each method: this adjusts the density estimates and their SEs for the smaller number of annotations. Standard errors for the estimated parameters were calculated from 500 bootstrap iterations, in which detection histories are generated assuming $D=\hat{D}$, and models fitted repeatedly (Stevenson et al., 2015).

As a second metric, we used event-based $F_{1}$ score (Serizel et al., 2018). All recordings were annotated manually, and compared with all detections from each algorithm. Detections that overlap a manual annotation were counted as true positives, while detections that did not overlap any annotation, or multiple ones overlapping a single annotation, were counted as false positives, etc. The $F_{1}$ score and recall (true pos./annotated pos.) were calculated from these using standard definitions (Serizel et al., 2018). 


\section{Bittern survey}

The Australasian bittern (Botaurus poiciloptilus) is an endangered bird species, ranked as nationally critical in New Zealand. Its populations are monitored acoustically, as the birds are very difficult to sight, but the males produce a distinctive low frequency (around $150 \mathrm{~Hz}$ ) 'boom' call, typically repeated in trains of several 'booms'. A bittern survey was conducted near Lake Ellesmere, New Zealand, on 6 and 7 December 2019. Between 22:00-23:00 and 04:00-05:00, a recording containing bittern calls was played on two speakers to solicit responses, and 7 acoustic recorders (AR4, Department of Conservation) were deployed in the surrounding area (Supplementary Figure S3).

Three prefilter methods were trained as described above, using approx. 30 min of audio from other surveys, and applied to this data. Since the main unit for our downstream analysis is a train of 'booms', which may last for several seconds, we merged any consecutive detections with gaps under $3 \mathrm{~s}$. We reviewed the calls by spectrogram inspection and playback in AviaNZ; segments were split into $3 \mathrm{~s}$ fragments for this process. The SCR and $F_{1}$ metrics for each method were then calculated as described earlier.

\section{Little spotted kiwi survey}

The little spotted kiwi (Apteryx owenii) is a near-threatened bird species, confined to several intensively managed areas. The male call is a sequence of $10-30$ short whistling syllables at around $2 \mathrm{kHz}$ to $3 \mathrm{kHz}$ pitch (see also Figure 1). About 200 individuals are present in Zealandia, a 225 ha wildlife sanctuary in Wellington, New Zealand. A passive acoustic survey was conducted in this sanctuary using 8 SM-2 recorders (Wildlife Acoustics, Inc.), placed as shown in Supplementary Figure S4. We use recordings made on the night of November 5-6, 2018, between 22:0000:00 and 02:00-04:00, when the kiwi call rate is presumed higher.

The data was analysed similarly as for bittern: three prefilters were used to detect the male calls, with approximately $30 \mathrm{~min}$ of audio data collected in other surveys used for training their parameters. Detections shorter than $5 \mathrm{~s}$ were removed from all outputs. Since the kiwi call is relatively long, this greatly reduces the review effort by excluding other events of similar pitch, such as kaka (a parrot, Nestor meridionalis) calls. The remaining detections were manually reviewed and used for evaluation by SCR, or compared with the ground truth annotations for $F_{1}$ scores. 


\subsection{Application to DCASE data}

As an example of bioacoustic detection on a standard benchmark dataset, we use DCASE 2018 Task 4 data (Serizel et al., 2018). It consists of 10-second audio clips from Youtube videos with several event classes labelled. The goal is to accurately detect both the class and the timestamp of each event.

We tested the compared methods on the "Cat" class, as it represents biophonic tonal sounds, which is the typical use case of our method (in contrast to other classes in this dataset such as "Frying"). In the original task, training used a large, but weakly labelled subset of clips. To train our method, we manually timestamped 12 clips from this set: 5 were used to select the wavelet nodes and maximum call length $l$, and 7 clips were used to tune the detection threshold parameter to obtain around $90 \%$ recall (at $1 \mathrm{~s}$ resolution). The clips were selected randomly, but manually verified to contain typical cat meowing sounds. When applying the wavelet filter, the window size was set to $0.1 \mathrm{~s}$ instead of the $1 \mathrm{~s}$ defined in the original publication, because the DCASE metric requires more precise annotation positions.

The data also prominently features human speech, which occupies similar frequency and time range as the target cat sounds. To distinguish these events, we supplement our detector with a simple classifier. The classifier uses frame-level features - 24 mel-frequency cepstral coefficients (MFCCs) and zero-crossing rate (ZCR) as a proxy of pitch. These were extracted using the librosa library (McFee et al., 2015) with default parameters. The first MFCC was dropped, and all features standardised to zero mean, unit SD for each audio file, and minimal smoothing over time applied (3-frame uniform filter). These features and all pairwise interactions between them were used to build a logistic regression with LASSO penalty, which classifies each frame as cat or non-cat. This model was trained on the 12 cat clips that we annotated earlier, plus 10 non-cat clips from the same DCASE training dataset (note that for these the weak labels are sufficient to determine the frame-level ground truth).

We processed the entire DCASE evaluation dataset with this workflow: the clips were first segmented using one of the detection methods (median clipping or wavelet changepoints), and each segment was then retained if the logistic regression classified at least $10 \%$ of its frames as positive. (As a sensitivity analysis, we replaced the acceptance rule with "average predicted log-odds above 0"; the results were similar and are not shown here.) We report the results obtained with the a priori segment-level threshold of 0 , but also repeated the analysis with different thresholds and generated a precision-recall curve, shown in Supplementary Figure 
S5. The results were evaluated as defined in the DCASE task, i.e. by event-based $F_{1^{-}}$ score, where true positives require both the onset and offset of a reported segment to be within $0.2 \mathrm{~s}$ of the provided timestamps. For comparison, $F_{1}$ scores of other submissions on the same data were retrieved from the official task leaderboard.

\section{Experimental results}

\subsection{Bioacoustic surveys}

\section{Bittern}

In the 14 hours of audio analysed, the three methods produced varying numbers of detections for review. The smallest number of detections (1505) was produced by the proposed changepoint method. The wavelet filter reported 3469 detections, so only $43 \%$ of these were processed further to equalize the review effort, and median clipping reported 2307 detections (65\% retained). After review and postprocessing, 204 trains of 'booms' were identified in the annotations from the proposed method. The other two methods resulted in fewer cues (138 for median clipping and 93 for wavelet filter), approximately corresponding to the fraction of annotations processed. The proposed method is also computationally efficient, producing outputs faster than either of the other two methods.

The results of fitting an SCR model to this data are shown in Table 1. As expected, the estimated detection parameters were similar for all methods (the estimated detection radius $g_{0}$ was 1 in every case). The density estimates were also within 1 SE of each other. However, the precision clearly reflects the differences in efficiency of the tested methods, with changepoint detection providing a two-fold improvement in SE, compared to the worst of the three methods (which differed in the two cases).

The proposed method is also ranked best by $F_{1}$ score (Table 1, bottom). Detailed comparison of manual and automatic annotations revealed that all methods had similar recall, detecting close to $90 \%$ of the true calls, and differed mainly in the number of false alarms (most of these were non-target auditory events, such as farm animal sounds or human speech). 


\section{Little spotted kiwi}

In the survey of little spotted kiwi, the proposed method again produced the smallest number of detections to be reviewed: 1168, compared to 1346 using the wavelet filter, of which $87 \%$ were reviewed, and 2479 using median clipping, 47\% reviewed. In this data, the review found 116 kiwi call instances for the proposed method, 100 calls for wavelet filter and 52 calls for the median clipping. Note that these methods are segmenting every sound, so it would be expected that most sounds are not the target species.

The SCR results are shown in Table 1. The proposed changepoint method again produced the highest precision, with about two-fold smaller SE of the density estimate than the other methods. Estimates of the parameter $\sigma$ suggest that median clipping may be slightly more sensitive to distant calls, but any benefit was negated by a much higher false alarm rate. Estimates of the density $\hat{D}$ were again similar across all methods.

The same ranking is produced by comparing the $F_{1}$ scores (Table 1 , bottom). The recall for all methods was similar, between 81-89\%. The differences were mainly in the number of false alarms, which is captured by both metrics. Overall, the $F_{1}$ scores were much lower than for the bittern survey, but this does not indicate that this call is more complex: as kiwi calls were rarer, the number of true positives was small, while the amount of interference was similar in both datasets. This highlights the broader issue that standard metrics may not capture applicationspecific nuances that are important in practice.

Table 1: Precision of acoustic surveys using different call detection methods: wavelet filter (WF), median clipping (MC), and the changepoint detection method (CD) proposed in this paper. The density is the intensity $D$ of the call emission process per hectare (ha), while $\sigma$ is a parameter controlling the detection function, as defined in Methods 3.2. Also shown is the event-based $F_{1}$ score based on matches between manual annotations and each method's output. The detector with the best metric values (lowest SE, or highest $F_{1}$ ) is highlighted for each survey.

\begin{tabular}{l|rrr|rrr}
\hline & \multicolumn{3}{|c|}{ Bittern } & \multicolumn{3}{c}{ Kiwi } \\
& WF & MC & CD & WF & MC & CD \\
\hline$\sigma(\mathrm{m})$ & 290 & 293 & 265 & 216 & 290 & 209 \\
SE of $\sigma$ & 97.5 & 99.6 & 90.6 & 18.7 & 48.7 & 15.6 \\
Density (calls/ha) & 0.59 & 0.45 & 0.53 & 0.69 & 0.61 & 0.59 \\
Density SE & 0.68 & 0.40 & $\mathbf{0 . 2 9}$ & 0.14 & 0.26 & $\mathbf{0 . 1 0}$ \\
$F_{1}(\%)$ & 33.5 & 45.6 & $\mathbf{6 3 . 7}$ & 19.8 & 12.5 & $\mathbf{2 3 . 5}$ \\
\hline
\end{tabular}




\subsection{DCASE data}

In the DCASE evaluation data, we tested the use of the wavelet filter, median clipping and changepoint detector as prefiltering algorithms. On their own these methods accurately detected between $19.9 \%$ (WF) and $37.0 \%$ (CD) of the 246 cat vocalizations present, but also produced many false positives (these include mostly speech, but also cat sounds that were marked with $>0.2 \mathrm{~s}$ error at the boundaries). Some of the false negatives were unusual cat sounds, highlighting the limitations of using a small training set. The resulting $F_{1}$ scores are shown in Table 2 . When these prefilters are combined with a basic classifier, most of the false positives were removed, with little loss in recall (18-31\%). The proposed changepoint method was the best of the three prefilter options, achieving an $F_{1}$ score of $19.6 \%$.

While the scores achieved by the tested method appear low, comparison with the DCASE leaderboard reveals that this was a difficult task (Table 2). Out of the 51 submissions in the competition, the best score achieved for the cat class was $25 \%$, while the competition winner and the organizers' baseline achieved only about $3 \%$ $F_{1}$ score and are outperformed by the proposed method, even without any classifier. Note that all the benchmark methods are complex CNN models, trained on a much larger set of 1600 weakly labelled and 14,000 unlabelled clips. This suggests that modern sound event detection can still benefit from improved methods for defining segment boundaries.

Table 2: Performance of three prefiltering methods (changepoint detection CD, wavelet filter WF, median clipping MC), prefilters combined with an MFCC classifier, and benchmark methods on the DCASE evaluation dataset. $F_{1}(c a t)$ is the event-level $F_{1}$ score for the "Cat" class labels. Benchmark methods are the top scoring submissions in DCASE 2018, based on reported results over all classes, or for the cat class.

\begin{tabular}{lc}
\hline Method & $F_{1}($ cat $)$ \\
\hline DCASE2018 baseline & $2.9 \%$ \\
LJK_PSH_task4_4 (1st overall) & $3.6 \%$ \\
Liu_USTC_task4_4 (2nd overall) & $20.3 \%$ \\
PELLEGRINI_IRIT_task4_2 (1st in class) & $25.3 \%$ \\
Liu_USTC_task4_1 (2nd in class) & $23.1 \%$ \\
WF & $4.8 \%$ \\
MC & $4.5 \%$ \\
CD (proposed) & $4.9 \%$ \\
WF + MFCC & $8.7 \%$ \\
MC + MFCC & $9.6 \%$ \\
CD + MFCC (proposed) & $19.6 \%$ \\
\hline
\end{tabular}




\section{Discussion}

Our results demonstrate that detection of bioacoustic sound events can be considerably improved by changepoint theory. The proposed method adapts a nuisancerobust changepoint detector, introduced in Juodakis and Marsland (2020), to analyse a wavelet representation of sound. The theoretical results presented here show that this method is consistent and robust to parameter mis-specification. These benefits were confirmed experimentally: in the field data, the proposed method produced fewer false positives and substantially improved acoustic survey efficiency, compared to other detectors used in bioacoustics. In more complex tasks such as the DCASE challenge, combining the proposed method with a simple classifier led to performance almost equal to state-of-the-art detectors while using much less training data (22 instead of 1600 clips, albeit with timestamped annotations).

A major challenge in developing sound analysis tools is generalization to different types of signals and noises (Priyadarshani et al., 2018). We observe this in our survey data as well: while the wavelet filter worked well on kiwi, for which it was originally developed (Marsland et al., 2019), median clipping was much more successful for detecting the short, contiguous calls of bittern. Huge variability is also seen for more advanced detectors in DCASE 2018 results, with leaders scoring almost $50 \% F_{1}$ for some classes and $4 \%$ for others (Serizel et al., 2018); fully relying on deep learning makes it is especially difficult to analyse the causes of such differences. Even for seemingly similar noises, such as rain recorded in different locations, the performance of detectors can vary dramatically (Metcalf et al., 2020). Thus, a very large and diverse validation dataset would be needed to empirically demonstrate a consistent advantage for any method. Instead, by focusing on the theoretical performance analysis, we have demonstrated that our method can accurately detect any events that appear as a change of scale in wavelet coefficients.

However, the theoretical changepoint framework makes some strong assumptions which can limit its validity in practice. Increase in energy, while still commonly used as the main feature for prefiltering of cues in bioacoustics (e.g. Barlow et al. (2021)), will not capture all sounds, especially in complex acoustic scenes where the background may change even during a signal event. Secondly, many non-target events will appear similar when using only simple features, as seen for example with wind noise in the field surveys here. Appropriate choice of sound representation is also necessary to satisfy the statistical model of the background process. We use a wavelet basis as it allows some frequency band selectivity and reduces 
correlations in the noise (Wornell, 1993). This probably does not fully satisfy the assumptions, but it appears that changepoint models are robust and still work with clearly non-Gaussian data, at least for stronger changes (Juodakis and Marsland, 2020). Alternatively, representations such as mel-frequency cepstral coefficients could be used, or the recordings can be initially denoised to help with these issues, for example, by applying spectral subtraction (Nelke and Vary, 2015). For tasks where our chosen model is severely inaccurate, the framework presented here can be a starting point for further method development and also help understand the strengths and weaknesses of different detectors.

Evaluating the success of detection empirically is another challenge, and various metrics could be used. Measures such as the $F_{1}$ score directly capture the agreement between the detections and expert annotations. However, maximizing these metrics does not always lead to more efficient monitoring, and the SCR metric used in the survey data was specifically developed to address that (Juodakis et al., 2021). By measuring the precision of the final estimate of interest, it captures the complete effects of various differences between methods that may be relevant in practice. Depending on the application, other measures can be used as well, such as detection range (Barlow et al., 2021). In any case, since the methods in our study were calibrated to differ mainly in their false alarm rates, most common metrics should rank them similarly.

The proposed changepoint prefilter can be extended to different applications with some simple changes. We used it as a monophonic method in our experiments. The training procedure is designed for homogeneous data representing a single type of sound, which allows it to identify the parameters efficiently from only several examples. Polyphonic detection can be obtained simply by analysing each recording with several detectors, trained on different sounds. For instance, the recall of the presented cat sound detection could be increased by adding detectors specific to purring or other types of vocalizations that were not represented in the current training set. The method can also be applied to non-tonal (impulsive) sounds, but with less selectivity, as it uses tonal properties to help distinguish different sounds. This nonetheless can be useful in tasks like bat click detection (Loeb et al., 2015), where fewer interfering noises are present.

In practice, it is likely that such prefilters will be followed by further classification of the outputs. This could be achieved by neural networks, but they require large amounts of annotated training data, which is often expensive to obtain. Investigating how various types of neural network can be informed by a better pre- 
filtering stage is an interesting avenue for future work. Finally, changepoint theory is developing rapidly, and may lead to other methods, even better suited to audio data; we hope that our findings will encourage further application of such principled methods to sound analysis.

\section{Software and data availability}

The proposed detector and the comparison methods are implemented as part of the open-source sound analysis software AviaNZ. The software and other analysis code are available at https://github.com/smarsland/AviaNZ/releases/ tag/CHP_v1. The settings used in the experiments in AviaNZ-compatible JSON format, as well as the audio data and produced annotations, are deposited at https : //doi.org/10.5281/zenodo. 4655178.

\section{Bibliography}

Aminikhanghahi, S. and Cook, D. J. (2016) A survey of methods for time series change point detection. Knowledge and Information Systems, 51, 339-367.

Barker, J., Watanabe, S., Vincent, E. and Trmal, J. (2018) The fifth 'CHiME' speech separation and recognition challenge: Dataset, task and baselines. In Proc. Interspeech 2018, 1561-1565.

Barlow, J., Fregosi, S., Thomas, L., Harris, D. and Griffiths, E. T. (2021) Acoustic detection range and population density of cuvier's beaked whales estimated from near-surface hydrophones. The Journal of the Acoustical Society of America, 149, 111-125.

Borchers, D. L., Stevenson, B. C., Kidney, D., Thomas, L. and Marques, T. A. (2015) A unifying model for capture-recapture and distance sampling surveys of wildlife populations. Journal of the American Statistical Association, 110, 195-204.

Castillo-Escario, Y., Ferrer-Lluis, I., Montserrat, J. M. and Jane, R. (2019) Entropy analysis of acoustic signals recorded with a smartphone for detecting apneas and hypopneas: A comparison with a commercial system for home sleep apnea diagnosis. IEEE Access, 7, 128224-128241.

Chang, X. and Stein, M. L. (2013) Decorrelation property of discrete wavelet transform under fixed-domain asymptotics. IEEE Transactions on Information Theory, 59, 80018013.

Dawson, D. K. and Efford, M. G. (2009) Bird population density estimated from acoustic signals. Journal of Applied Ecology, 46, 1201-1209. 
Droghini, D., Principi, E., Squartini, S., Olivetti, P. and Piazza, F. (2017) Human fall detection by using an innovative floor acoustic sensor. In Multidisciplinary Approaches to Neural Computing, 97-107. Springer International Publishing.

Fisch, A. T. M., Eckley, I. A. and Fearnhead, P. (2018) A linear time method for the detection of point and collective anomalies. arXiv preprint: 1806.01947.

Fryzlewicz, P. (2014) Wild binary segmentation for multiple change-point detection. The Annals of Statistics, 42, 2243-2281.

Gibb, R., Browning, E., Glover-Kapfer, P. and Jones, K. E. (2018) Emerging opportunities and challenges for passive acoustics in ecological assessment and monitoring. Methods in Ecology and Evolution, 10, 169-185.

Goëau, H., Kahl, S., Glotin, H., Planqué, R., Vellinga, W.-P. and Joly, A. (2018) Overview of BirdCLEF 2018: monospecies vs. soundscape bird identification. In CLEF (Working Notes), vol. 2125 of CEUR Workshops Proceedings. Avignon, France: CEUR-WS.org.

Hayashi, T., Komatsu, T., Kondo, R., Toda, T. and Takeda, K. (2018) Anomalous sound event detection based on WaveNet. In 26th European Signal Processing Conference (EUSIPCO). IEEE.

Hubert, P., Killick, R., Chung, A. and Padovese, L. R. (2019) A Bayesian binary algorithm for root mean squared-based acoustic signal segmentation. The Journal of the Acoustical Society of America, 146, 1799-1807.

Juodakis, J., Castro, I. and Marsland, S. (2021) Precision as a metric for acoustic survey design using occupancy or spatial capture-recapture. Environmental and Ecological Statistics, 28, 587-608.

Juodakis, J. and Marsland, S. (2020) Epidemic changepoint detection in the presence of nuisance changes. arXiv preprint: 2008.08240.

Killick, R., Fearnhead, P. and Eckley, I. A. (2012) Optimal detection of changepoints with a linear computational cost. Journal of the American Statistical Association, 107, 15901598.

Lasseck, M. (2013) Bird song classification in field recordings: Winning solution for NIPS4B 2013 competition. In Proceedings of 'Neural Information Processing Scaled for Bioacoustics: From Neurons to Big Data - NIP4B', 176-181.

- (2016) Improving bird identification using multiresolution template matching and feature selection during training. In CLEF2016 Working Notes, vol. 1609, 490-501. CEURWS.org.

LINZ (2008) Standard for New Zealand Geodetic Datum 2000 Projections (LINZS25002). Office of the Surveyor-General, Wellington, New Zealand.

Liu, Y. L., Yan, J., Song, Y. and Du, J. (2018) USTC-NELSLIP system for DCASE 2018 challenge Task 4. Tech. rep., DCASE2018 Challenge.

Loeb, S. C., Rodhouse, T. J., Ellison, L. E., Lausen, C. L., Reichard, J. D., Irvine, K. M., Ingersoll, T. E., Coleman, J. T., Thogmartin, W. E., Sauer, J. R., Francis, C. M., Bayless, M. L., 
Stanley, T. R. and Johnson, D. H. (2015) A plan for the North American Bat Monitoring Program (NABat). Tech. Rep. SRS-208, U.S. Department of Agriculture Forest Service, Southern Research Station.

Mallat, S. (2009) A Wavelet Tour of Signal Processing. Elsevier, Oxford.

Marsland, S., Priyadarshani, N., Juodakis, J. and Castro, I. (2019) AviaNZ: A future-proofed program for annotation and recognition of animal sounds in long-time field recordings. Methods in Ecology and Evolution, 10, 1189-1195.

McFee, B., Raffel, C., Liang, D., Ellis, D. P., McVicar, M., Battenberg, E. and Nieto, O. (2015) librosa: Audio and music signal analysis in python. In Proceedings of the 14th Python in Science conference, vol. 8, 18-25.

Merchan, F., Echevers, G., Poveda, H., Sanchez-Galan, J. E. and Guzman, H. M. (2019) Detection and identification of manatee individual vocalizations in Panamanian wetlands using spectrogram clustering. The Journal of the Acoustical Society of America, 146, 17451757.

Metcalf, O. C., Lees, A. C., Barlow, J., Marsden, S. J. and Devenish, C. (2020) hardRain: An $\mathrm{R}$ package for quick, automated rainfall detection in ecoacoustic datasets using a threshold-based approach. Ecological Indicators, 109, 105793.

Nelke, C. M. and Vary, P. (2015) Wind noise short term power spectrum estimation using pitch adaptive inverse binary masks. In 2015 IEEE International Conference on Acoustics, Speech and Signal Processing (ICASSP). IEEE.

Niu, Y. S., Hao, N. and Zhang, H. (2016) Multiple change-point detection: A selective overview. Statistical Science, 31, 611-623.

Pankajakshan, A., Bear, H. L. and Benetos, E. (2019) Polyphonic sound event and sound activity detection: A multi-task approach. In 2019 IEEE Workshop on Applications of Signal Processing to Audio and Acoustics (WASPAA). IEEE.

Phan, H., Koch, P., Katzberg, F., Maass, M., Mazur, R., McLoughlin, I. and Mertins, A. (2017) What makes audio event detection harder than classification? In 2017 25th European Signal Processing Conference (EUSIPCO). IEEE.

Priyadarshani, N., Marsland, S. and Castro, I. (2018) Automated birdsong recognition in complex acoustic environments: a review. Journal of Avian Biology, 49, jav-01447.

Priyadarshani, N., Marsland, S., Juodakis, J., Castro, I. and Listanti, V. (2020) Wavelet filters for automated recognition of birdsong in long-time field recordings. Methods in Ecology and Evolution, 11, 403-417.

Ramli, D. A. and Jaafar, H. (2016) Peak finding algorithm to improve syllable segmentation for noisy bioacoustic sound signal. Procedia Computer Science, 96, 100-109.

Robertson, H. and Colbourne, R. (2017) Kiwi best practice manual. Wellington, New Zealand: Department of Conservation.

Serizel, R., Turpault, N., Eghbal-Zadeh, H. and Shah, A. P. (2018) Large-scale weakly labeled semi-supervised sound event detection in domestic environments. In Proceed- 
ings of the Detection and Classification of Acoustic Scenes and Events 2018 Workshop (DCASE2018), 19-23.

Stevenson, B. C., Borchers, D. L., Altwegg, R., Swift, R. J., Gillespie, D. M. and Measey, G. J. (2015) A general framework for animal density estimation from acoustic detections across a fixed microphone array. Methods in Ecology and Evolution, 6, 38-48.

Truong, C., Oudre, L. and Vayatis, N. (2020) Selective review of offline change point detection methods. Signal Processing, 167, 107299.

Wang, X., Jiang, J., Duan, F., Liang, C., Li, C., Sun, Z., Lu, R., Li, F., Xu, J. and Fu, X. (2021) A method for enhancement and automated extraction and tracing of Odontoceti whistle signals base on time-frequency spectrogram. Applied Acoustics, 176, 107698.

Wornell, G. W. (1993) Wavelet-based representations for the $1 / \mathrm{f}$ family of fractal processes. In Proceedings of the IEEE, vol. 81, 1428-1450. IEEE.

Wright, D. L., Berchok, C. L., Crance, J. L. and Clapham, P. J. (2018) Acoustic detection of the critically endangered North Pacific right whale in the northern Bering Sea. Marine Mammal Science, 35, 311-326.

Yao, Y.-C. and Au, S. T. (1989) Least-squares estimation of a step function. Sankhyā (Statistics). The Indian Journal of Statistics. Series A, 51, 370-381.

Zeppelzauer, M., Hensman, S. and Stoeger, A. S. (2015) Towards an automated acoustic detection system for free-ranging elephants. Bioacoustics, 24, 13-29.

Zgank, A. (2019) Bee swarm activity acoustic classification for an IoT-based farm service. Sensors, 20, 21.

Zhao, Z. and Yau, C. Y. (2021) Alternating pruned dynamic programming for multiple epidemic change-point estimation. Journal of Computational and Graphical Statistics, 30, 808-821.

Zheng, C., Eckley, I. A. and Fearnhead, P. (2019) Consistency of a range of penalised cost approaches for detecting multiple changepoints. arXiv preprint: 1911.01716. 


\section{Supplementary material}

\section{A Convergence when estimating variance}

Our goal is to show that Algorithm 1 converges when estimating the variance parameter of a Gaussian distribution in data with changepoints. We have previously showed that this method can be used to estimate the location parameter of a symmetric distribution (Juodakis and Marsland, 2020). The proof here follows a similar structure. We will show that when short segments are considered, the expected direction of the iterative updates points towards the true parameter value. This can then be generalized to longer segments as described in Juodakis and Marsland (2020).

The estimation of background variance in the Algorithm can be expressed as the following recursive update, with $s_{t}^{2}=\sum_{i=1}^{t} x_{i}^{2} / t$ :

$$
s_{t+1}^{2}=s_{t}^{2}+\frac{1}{t+1}\left(x_{t+1}^{2}-s_{t}^{2}\right)
$$

This is a special case of the general online algorithm form:

$$
w_{t+1}=w_{t}-\gamma_{t} H\left(x_{t+1}, w_{t}\right)
$$

where $\gamma_{t}=1 /(t+1)$ is called the learning rate, and $H(x, w)=\left(w-x^{2}\right)$ is the update. Bottou (1998) analysed the convergence of such algorithms and established three conditions that are sufficient:

1. "convexity" - the expected value of the updates always points towards a single optimum $w^{*}$ :

$$
\forall \epsilon>0, \inf _{\left(w-w^{*}\right)^{2}>\epsilon}\left(w-w^{*}\right) \mathbb{E} H(x, w)>0 ;
$$

2. learning rate growth:

$$
\sum_{i=1}^{\infty} \gamma_{t}=\infty, \sum_{i=1}^{\infty} \gamma_{t}^{2}<\infty
$$

3. bounded variance of the updates:

$$
\mathbb{E} H(x, w)^{2} \leq A+B\left(w-w^{*}\right)^{2}, A, B \geq 0 .
$$

We will show that these conditions are satisfied for Algorithm 1 with $w^{*}=\theta_{0}^{2}$ 
(the true background variance), and therefore it converges towards this value. The following lemmas will be needed to replace Lemma 1 from Juodakis and Marsland (2020).

Lemma 1. Let $Y \sim \chi^{2}(1)$. Consider the variable $\tilde{Y}$, obtained by truncating $Y$ to an interval $(l ; u)$. Then, $\mathbb{E} \tilde{Y}=1$ if and only if $l$, u satisfy $f_{\chi^{2}}(l ; 3)=f_{\chi^{2}}(u ; 3)$.

Proof. We will use the shorthand $F$ and $f$ for $F_{\chi^{2}}$ and $f_{\chi^{2}}$, respectively. A formula for the $\alpha$-th moment of a (central) $\chi^{2}(p)$ variable truncated to $(l, u)$ is provided in Marchand (1996):

$$
\mathbb{E}(\tilde{Y})^{\alpha}=2^{\alpha} \frac{\Gamma(p / 2+\alpha)}{\Gamma(p / 2)} \frac{F(u ; p+2 \alpha)-F(l ; p+2 \alpha)}{F(u ; p)-F(l ; p)} .
$$

Marchand (1996) also provides the following recursion property, for $p \geq 3$ :

$$
F(y ; p)=F(y ; p-2)-2 f(y ; p)
$$

Substituting this into (S10), and applying the definition of the gamma function, we can obtain this special case for $\alpha=1, u \neq l$ :

$$
\begin{aligned}
\mathbb{E}(\tilde{Y}) & =p \frac{F(u ; p+2)-F(l ; p+2)}{F(u ; p)-F(l ; p)} \\
& =p \frac{F(u ; p)-2 f(u ; p+2)-F(l ; p)+2 f(l ; p+2)}{F(u ; p)-F(l ; p)} \\
& =p\left(1+2 \frac{f(l ; p+2)-f(u ; p+2)}{F(u ; p)-F(l ; p)}\right) .
\end{aligned}
$$

Clearly, when the truncation interval satisfies $f(l ; p+2)=f(u ; p+2)$, the fraction is 0 and $\mathbb{E}(\tilde{Y})=p$. The case for $p=1$ follows immediately.

Lemma 2. Assume $Y$ follows a distribution in the one-parameter exponential family, i.e., its pdf can be expressed in the form $f(y \mid \theta)=h(y) e^{\eta(\theta) T(y)-\psi(\theta)}$, or equivalently $f(y \mid \eta)=h(y) e^{\eta T(y)-\psi(\eta)}$. Then, for any interval $(l ; u)$ such that $l, u$ do not depend on $\theta$, the expected value of $Y$ truncated to this interval is increasing in $\eta$ :

$$
\frac{d \mathbb{E}(Y \mid Y \in(l ; u) ; \eta)}{d \eta}>0
$$

Proof. For the exponential family distributions:

$$
\frac{d \mathbb{E}(Y \mid \eta)}{d \eta}=\psi^{\prime \prime}(\eta)=\operatorname{Var}(Y \mid \eta) \geq 0
$$


with equality holding only for degenerate distributions.

The truncated variable $\tilde{Y}$ has the following pdf, supported over the interval $(l ; u)$ :

$$
\begin{aligned}
f(\tilde{y} \mid \theta) & =\frac{1}{F(u \mid \theta)-F(l \mid \theta)} h(\tilde{y}) e^{\eta T(\tilde{y})-\psi(\eta)} \\
& =h(\tilde{y}) \exp (\eta T(\tilde{y})-\psi(\eta)-\log (F(u \mid \theta)-F(l \mid \theta))) \\
& =h(\tilde{y}) \exp (\eta T(\tilde{y})-\tilde{\psi}(\eta))
\end{aligned}
$$

Therefore, the truncated variable distribution also belongs to the exponential family, and (S11) applies.

Now, we can prove the following result.

Theorem 1. Consider the problem of epidemic changes in variance, i.e., data $x_{0: n}$ generated from model (2), with $f(x \mid \theta)=1 / \sqrt{2 \pi \theta^{2}} \exp \left(-\frac{x^{2}}{2 \theta^{2}}\right)$. The background variance $\theta_{0}^{2}$ is unknown. Denote by $w_{t}$ the estimate of $\theta_{0}$ obtained by analysing $x_{0: t}$ using Algorithm 1. The sequence $\left\{w_{t}\right\}$ converges to $\left(\theta_{0}-\epsilon, \theta_{0}+\epsilon\right)$ almost surely, with $\epsilon \rightarrow 0$ as the number of background points between successive segments $n \rightarrow \infty$.

Proof. W.l.o.g. we will assume $\mu_{0}=0, \sigma_{0}^{2}=1$.

We will first show the case when maximum segment length $l=1$, and assuming no signal points are misclassified as background. At time $t$, the point $x_{t+1}$ will be accepted as background, and thus used to update $s^{2}$, if it is in this region:

$$
A_{1}=\left\{x_{t+1}: C\left(x_{t+1} ; s_{t}^{2}\right)<\min _{\theta} C\left(x_{t+1} ; \theta\right)+\beta\right\}
$$

Based on the definition of $C$, the acceptance rule can be expressed as:

$$
\begin{gathered}
-\log f_{N}\left(x_{t+1} ; 0, s_{t}^{2}\right)<-\log f_{N}\left(x_{t+1} ; 0, x_{t+1}^{2}\right)+\beta \\
\Rightarrow f_{N}\left(x_{t+1} ; 0, s_{t}^{2}\right)>f_{N}\left(x_{t+1} ; 0, x_{t+1}^{2}\right) e^{-\beta},
\end{gathered}
$$

where $f_{N}$ denotes the Gaussian pdf. Define $y=\left(x_{t+1} / s_{t}\right)^{2}$. Now, the acceptance rules can be expressed in terms of $f_{\chi^{2}}(\cdot ; p)$, the pdf of a $\chi^{2}$-distributed r.v. with $p$ 
degrees of freedom:

$$
\begin{aligned}
f_{N}\left(x_{t+1} ; 0, s_{t}^{2}\right) & =\frac{1}{\left|s_{t}\right|} f_{N}(\sqrt{y} ; 0,1) \\
& =\frac{1}{\left|s_{t}\right|} \sqrt{y} f_{\chi^{2}}(y ; 1) \\
& =\left|\frac{\sqrt{y}}{s_{t}}\right| \frac{1}{2^{1 / 2} \Gamma(1 / 2)} y^{-1 / 2} e^{-y / 2} \\
& =\left|\frac{\sqrt{y}}{s_{t}}\right| \frac{1}{2^{3 / 2} \Gamma(3 / 2)} y^{1 / 2-1} e^{-y / 2} \\
& =\left|\frac{1}{s_{t}}\right| y^{-1 / 2} f_{\chi^{2}}(y ; 3)
\end{aligned}
$$

Here we used change of variables to obtain the first two equalities, and the definition of $f_{\chi^{2}}$ for the rest. Also, we have:

$$
\begin{aligned}
f_{N}\left(x_{t+1} ; 0, x_{t+1}^{2}\right) & =\frac{1}{\left|x_{t+1}\right|} f_{N}(1 ; 0,1) \\
& =\frac{c}{\left|x_{t+1}\right|}, \text { with some } c>0 .
\end{aligned}
$$

Substituting these two results into (S12), the acceptance condition is, with some $c_{2}>0$ :

$$
\begin{aligned}
A_{1} & =\left\{y: \frac{y^{-1 / 2}}{\left|s_{t}\right|} f_{\chi^{2}}(y ; 3)>\frac{c_{2}}{\left|x_{t+1}\right|}\right\} \\
& =\left\{y: f_{\chi^{2}}(y ; 3)>c_{2}\right\} .
\end{aligned}
$$

Since $f_{\chi^{2}}$ is unimodal, this means that the acceptance region on $y$ is an interval $(l ; u)$ with endpoints that satisfy $f_{\chi^{2}}(l ; 3)=f_{\chi^{2}}(u ; 3)=c_{2}$.

Recall that the "convexity" condition (S7) requires that:

$$
\mathbb{E}\left(s_{t}^{2}-x_{t+1}^{2} \mid x_{t+1} \in A_{1}\right)=s_{t}^{2}\left(1-\mathbb{E}\left(y \mid x_{t+1} \in A_{1}\right)\right)>0,
$$

or equivalently

$$
\mathbb{E}\left(y \mid x_{t+1} \in A_{1}\right)<1
$$

when $s_{t}^{2}>\sigma_{0}^{2}$, and vice versa.

Since $x$ is Gaussian, $y$ is distributed as $\chi^{2}(1)$ when $s_{t}^{2}=\theta_{0}^{2}$, or in the general case $\Gamma\left(1 / 2, s_{t}^{2} / 2\right)$ using the rate parameterization (i.e. $\left.\alpha=1 / 2, \beta=s_{t}^{2} / 2\right)$. Thus, when $s_{t}^{2}=\theta_{0}^{2}$, Lemma 1 can be applied with the truncation limits established earlier, and 
we have:

$$
\mathbb{E}\left(y \mid x_{t+1} \in A_{1}\right)=1 .
$$

So it suffices to show that:

$$
\frac{d \mathbb{E}\left(y \mid x_{t+1} \in A_{1}\right)}{d s^{2}}<0
$$

for any $s^{2}$.

Using the canonical form of the gamma distribution, $\eta(\beta)=-\beta=-s^{2} / 2$, so:

$$
\frac{d \mathbb{E}\left(y \mid x_{t+1} \in A_{1}\right)}{d s^{2}}=\frac{d \mathbb{E}\left(y \mid y \in A_{1} ; \eta\right)}{d \eta} \frac{d \eta}{d s^{2}}=-\frac{1}{2} \frac{d \mathbb{E}\left(y \mid y \in A_{1} ; \eta\right)}{d \eta}<0
$$

by Lemma 2 . This proves (S15) and in turn (S7).

We see from the formula of the recursive update that the learning rate is $\gamma_{t}=$ $1 /(t+1)$, and therefore condition (S8) is satisfied. Because $A_{1}$ is bounded, the variance of the updates is finite, i.e. condition (S9) holds. Therefore, for $l=1$, over background points $w_{t} \rightarrow \theta_{0}^{2}$.

The rest of the proof is identical to that of Theorem 1 in Juodakis and Marsland (2020) and not reproduced here. Briefly, using properties of the exponential distribution family, the convergence obtained so far can be extended to the general case of longer segments $(l>1)$. Then, to account for misclassifications at times $T_{1}, T_{2}, \ldots$, define a new sequence $\left\{w_{i}^{\prime}\right\}=\left\{w_{T_{1}-1}, w_{T_{2}-1}, \ldots\right\}$. Over the background points between these misclassifications, the convergence result applies, and therefore the estimates recover from the perturbation. At finite $n$, the recovery is limited by some $\epsilon$ determined by the misclassification strength and frequency. Thus, $\left\{w_{i}^{\prime}\right\}$ satisfies the convergence conditions (S7)-(S9), and therefore converges to a region $\left(\theta_{0}^{2}-\epsilon, \theta_{0}^{2}+\epsilon\right)$. As $n \rightarrow \infty, \epsilon \rightarrow 0$.

\section{B Consistency of nuisance-robust detection}

To discuss the consistency of a segmentation method, it is convenient to consider separately the positions where a changepoint (of any type) was detected, and the reported types of segments. Therefore, we will use more explicit notation than in the main text: we will denote the true segmentation with a pair $T, E$, and its estimate (such as produced by the detection algorithm) with $\hat{T}, \hat{E}$. The first element contains only the positions of the changes, i.e. $\hat{T}=\operatorname{sort}\left(\hat{s} \cup \hat{e} \cup \hat{s}_{N} \cup \hat{e}_{N}\right)$. The pairing of these changes and the type of each segment (signal or nuisance) are stored in 
$\hat{E}:$

$$
\left.\hat{E}=\left\{\underset{0 \leq i \leq|T|+1}{\operatorname{argmin}}\left|t_{i}-\hat{s}\right|, \underset{0 \leq j \leq|T|+1}{\operatorname{argmin}}\left|t_{i}-\hat{e}\right|, \mathbb{1}(\hat{e}-\hat{s}<l) \mid \hat{s}, \hat{e}\right)\right\} .
$$

Thus for example $(1,2,0)$ indicates a segment starting close to $t_{1}$, ending close to $t_{2}$, of nuisance type; see also Figure S2. Since the start and end of the data series may also be used as segment start/end points, $t_{0}=0$ and $t_{|T|+1}=n$ are also added.

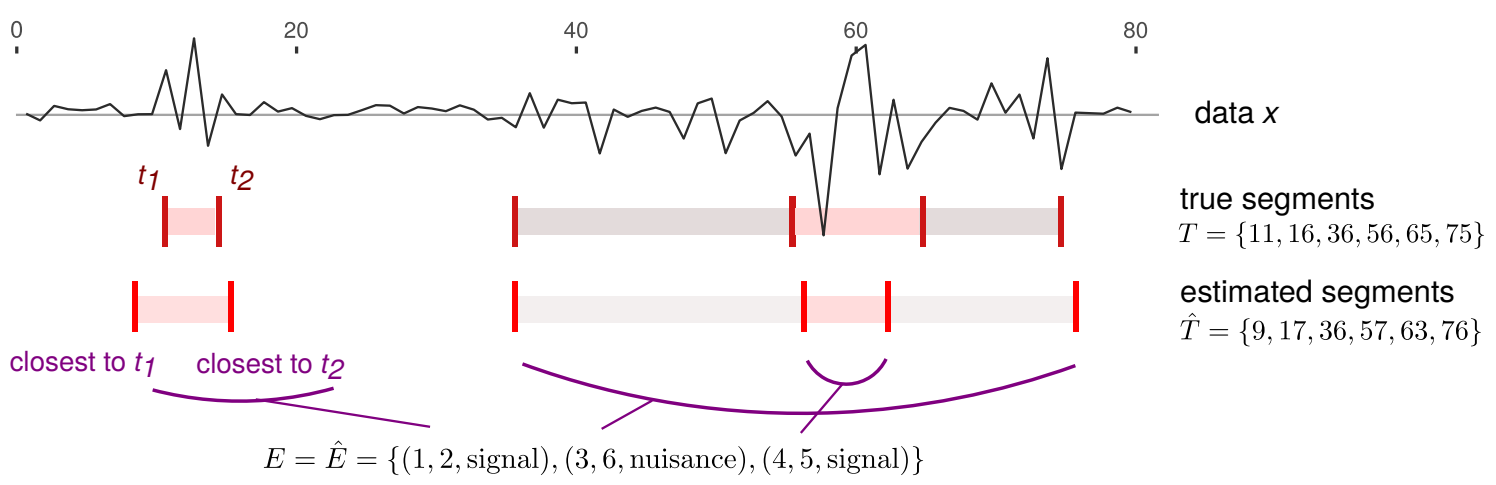

Figure S2: Visualizing the segment notation used in the consistency proofs. Assume the data contains three true segments: a signal segment at $(11,16)$, a nuisance segment at $(36,75)$, which is overlapped by another signal segment $(56,65)$. This corresponds to six changepoints (red bars). A detection algorithm outputs three pairs of segments, shown below. In our notation, $T$ contains only the changepoint positions, while $E$ indicates their assignment into segments. In a consistent segmentation, segment assignment must be accurate $(\hat{E}=E)$, but the positions $T$ and $\hat{T}$ are allowed to differ slightly.

In addition to the fitted cost of an epidemic model $\hat{C}^{N S}(x, T, E, \beta)$ defined in (5), we will denote by $C^{N S}(x, T, E, \beta)$ the analogous cost with true $\theta$ values $\left(\hat{C}^{N S}\right.$ is based on realised segment variances). We will also need the cost $C^{c l}\left(x_{0: n}, T, \beta\right)$ corresponding to the classical changepoint model with positions $T$ and true values of $\theta$ :

$$
C^{c l}\left(x_{0: n}, T, \beta\right)=\sum_{i=0}^{|T|} C\left(x_{t_{i}: t_{i+1}} ; \theta_{i}\right)+|T| \beta
$$

and its fitted analog $\hat{C}^{c l}\left(x_{0: n}, T, \beta\right)$. (Again using the convention $t_{0}=0, t_{|T|+1}=n$.)

We assume that the penalty in all cost functions takes the strengthened BIC or SIC form: $\beta=\alpha \log (n)^{1+\delta}$, with some user-chosen constants $\alpha>0, \delta>0$. Finally, we assume in this section that the global background parameter $\theta_{0}$ is known.

Our proof is based on consistency of classical changepoint detection, established previously. Specifically, we will use the following results:

Lemma 3 (Theorem 1, (Fisch et al., 2018)). Let $x_{0: n}$ be Gaussian data with true changepoints at positions $T=\left\{t_{1}, t_{2}, \ldots\right\}$. Assume (6) holds. We say that a segmen- 
tation $\hat{T}=\left\{\hat{t}_{1}, \hat{t}_{2}, \ldots\right\}$ is consistent if:

$$
|\hat{T}|=|T|, \forall i:\left|\hat{t}_{i}-t_{i}\right|<\frac{A}{\min \left(\Delta_{i}, \Delta_{i}^{2}\right)} \log (n)^{1+\delta}
$$

for some constant A. Denote by $\theta$ the vector of true parameters for each segment, and $\hat{\theta}$ their estimates. There exists an event $\mathscr{A}$ with $P(\mathscr{A}) \geq 1-B n^{-\epsilon}$ such that given $\mathscr{A}$ and sufficiently large $n$, for any inconsistent segmentation $T^{\prime}$ :

$$
\hat{C}^{c l}\left(x_{0: n}, T^{\prime}, \beta\right)>C^{c l}\left(x_{0: n}, T, \beta\right)+K_{1} \alpha \log (n)^{1+\delta} .
$$

We also have, as a corollary of consistency:

Lemma 4 ((Fisch et al., 2018)). There exists a constant $K_{2}$, such that given $\mathscr{A}$, for all $n>n_{0}$ :

$$
\hat{C}^{c l}\left(x_{i: j}, T, \beta\right)>C^{c l}\left(x_{i: j}, T, \beta\right)-K_{2} \log (n)
$$

We can now prove the consistency theorem.

Theorem 2. Let data $x_{0: n}$ be generated from the nuisance-signal changepoint model in (4), with $f(x \mid \theta)=1 / \sqrt{2 \pi \theta^{2}} \exp \left(-\frac{x^{2}}{2 \theta^{2}}\right)$, and additive signal and noise: $h\left(\theta^{S}, \theta^{N}\right)=$ $\sqrt{\left(\theta^{S}\right)^{2}+\left(\theta^{N}\right)^{2}}$. Set $\beta=\alpha \log (n)^{1+\delta}$. Assume that the length bound $l$ is chosen so that $l-\max \left|s_{i}-e_{i}\right| \geq \log (n)^{1+\delta_{2}}$ and $\min \left|s_{j}^{N}-e_{j}^{N}\right|-l \geq \log (n)^{1+\delta_{2}}$ for some $\delta_{2}>\delta$. If (6) holds, then minimisation of $\hat{C}^{N S}$ gives consistent estimates $\hat{T}, \hat{E}$, i.e., there exist constants $A, B, \epsilon$ and $n_{0}$, such that for $n>n_{0}$ :

$$
P\left(\hat{E}=E, \forall i \in 1, . .,|T|:\left|\hat{t}_{i}-t_{i}\right|<\frac{A}{\min \left(\Delta_{i}, \Delta_{i}^{2}\right)} \log (n)^{1+\delta}\right) \geq 1-B n^{-\epsilon}
$$

Proof. We will first show the consistency of estimated positions $\hat{T}$, and then the accuracy of assignments $\hat{E}$.

Consistency of changepoint positions. Note that the cost defined by our model can be compared to a classical changepoint model cost with a different penalty value. Using $\hat{\theta}$ to indicate the parameter values fitted under constraints imposed 
by $\hat{E}$ :

$$
\begin{aligned}
\hat{C}^{N S}\left(x_{0: n}, \hat{T}, \hat{E}, \beta\right) & =\sum_{i=0}^{|\hat{T}|} C\left(x_{t_{i}: t_{i+1}} ; \hat{\theta}_{i}\right)+\frac{|\hat{T}|}{2} \beta \\
& \geq \sum_{i=0}^{|\hat{T}|} \min _{\theta} C\left(x_{t_{i}: t_{i+1}} ; \theta\right)+|\hat{T}| \beta / 2 \\
& \geq \hat{C}^{c l}\left(x_{0: n}, \hat{T}, \beta / 2\right)+\frac{\beta}{2} \sum_{i=0}^{|\hat{T}|} \mathbb{1}\left(t_{i}+1=t_{i+1}\right) .
\end{aligned}
$$

Similarly,

$$
\hat{C}^{N S}\left(x_{0: n}, T, E, \beta\right) \leq C^{N S}\left(x_{0: n}, T, E, \beta\right)=C^{c l}\left(x_{0: n}, T, \beta / 2\right),
$$

because using the maximum likelihood $\theta$ estimates can only reduce the likelihoodbased cost.

Combining (S17) and (S18), we have:

$$
\hat{C}^{N S}\left(x_{0: n}, \hat{T}, \hat{E}, \beta\right)-C^{N S}\left(x_{0: n}, T, E, \beta\right) \geq \hat{C}^{c l}\left(x_{0: n}, \hat{T}, \beta / 2\right)-C^{c l}\left(x_{0: n}, T, \beta / 2\right)+K_{3} \beta
$$

for some constant $K_{3} \geq 0$.

Assume that $\hat{T}$ is inconsistent. Then, by Lemma 3, there exists an event $\mathscr{A}$, with $P(\mathscr{A}) \geq 1-B n^{-\epsilon}$, and $n_{0}$ such that for $n>n_{0}$ :

$$
\hat{C}^{c l}\left(x_{0: n}, \hat{T}, \beta / 2\right)-C^{c l}\left(x_{0: n}, T, \beta / 2\right)>0 .
$$

Therefore, with the same probability:

$$
\hat{C}^{N S}\left(x_{0: n}, \hat{T}, \hat{E}, \beta\right)>\hat{C}^{N S}\left(x_{0: n}, T, E, \beta\right),
$$

i.e., there is at least one segmentation $(T)$ with a cost lower than that of any segmentation with inconsistent positions. This contradicts the definition of $\hat{T}$ as the segmentation with minimal cost, and so $\hat{T}$ must be consistent.

Accuracy of segment type assignment. We will complete the proof by showing that when $\hat{T}$ is consistent, inaccurate assignment of these changepoints into pairs would also incur a high cost, and therefore $\hat{E}=E$.

Let $\operatorname{deg}(i)$ be the number of segments starting or ending near $t_{i}$ : $\operatorname{deg}(i)=$ $\sum\{\mathbb{1}(a=i \cup b=i) \mid(a, b, \cdot) \in \hat{E}\}$. (This notation is to emphasize that the segment 
pairs resemble edges on a graph over $0, \ldots,|T|+1$.)

Lemma 5. In the $\hat{E}$ that is estimated by minimising $\hat{C}^{N S}, \operatorname{deg}(i)=1$ for all $i \in$ $1, \ldots,|T|$, and $\operatorname{deg}(0)=0, \operatorname{deg}(|T|+1)=0$.

Proof. Firstly, choosing sufficiently large $n$ ensures that $\operatorname{deg}(i)>0$ for each $i \in$ $1, \ldots,|T|$ : otherwise, if $\hat{t}_{i}$ is closest not to $t_{i}$ but to $t_{j}, j \neq i$, we can choose $n$ large enough that $\left|\hat{t}_{i}-t_{i}\right|$ would violate the consistency of $\hat{T}$. Since $E$ by construction is just a partition of elements in $T$ into pairs, $|E|=|T| / 2$. Now, assume that $\operatorname{deg}(0)>0$, $\operatorname{deg}(|T|+1)>0$, or $\exists i: \operatorname{deg}(i)>1$. In either case, $|\hat{E}| \geq|T| / 2+1$, since at least one additional segment is needed, compared to the true segmentation.

But then applying Lemma 4, there exists $n_{0}$, such that for $n>n_{0}$ :

$$
\begin{aligned}
\hat{C}^{N S}\left(x_{0: n}, \hat{T}, \hat{E}, \beta\right)>C^{c l}\left(x_{0: n}, T, \beta / 2\right)+\beta(|\hat{E}|-|T| / 2)-K_{2} \log (n)> & \\
& >C^{N S}\left(x_{0: n}, T, E, \beta\right) \geq \hat{C}^{N S}\left(x_{0: n}, T, E, \beta\right),
\end{aligned}
$$

because $\beta>\mathscr{O}(\log (n))$. This contradicts the definition that $\hat{T}, \hat{E}$ has minimal cost, therefore $\operatorname{deg}(0)=\operatorname{deg}(|T|+1)=0, \operatorname{deg}(i) \leq 1$.

Lemma 6. Let $p_{2}=x_{\hat{t}_{a}: t_{b}}$ be a background piece, i.e., $(a, b, \cdot) \notin E$. Then any $E^{\prime}$ where $p_{2}$ is classified as a segment overlapping a nuisance, i.e., $(a, b, \cdot) \in E^{\prime},\left(a^{\prime}, b^{\prime}, \cdot\right) \in$ $E^{\prime}, a^{\prime}<a<b<b^{\prime}$, will not be optimal:

$$
\hat{C}^{N S}\left(x_{0: n}, T, E^{\prime}, \beta\right)>\hat{C}^{N S}\left(x_{0: n}, T, E, \beta\right)
$$

Proof. This could be derived from the construction of $\mathscr{A}$ presented in Fisch et al. (2018), but we will show it as a corollary of the already established consistency results. We will assume $\hat{\theta}_{1}>\theta_{0}$, (recall that $\theta_{0}$ is the background parameter), as the other case is symmetrical.

First, note that because the likelihood of $f$ is unimodal, the segment cost $C$ based on that likelihood is increasing in $|\hat{\theta}-\theta|$.

Denote $p_{1}=\left(a^{\prime}, b^{\prime}\right) \backslash p_{2}$, and let $\hat{\theta}_{1}, \hat{\theta}_{2}$ be the realized parameters of $p_{1}, p_{2}$. Assume for now $\hat{\theta}_{2}<\hat{\theta}_{1}$.

Then the cost of $T, E^{\prime}$ exceeds the cost of an inconsistent segmentation $T_{-p 2}$ 
which has no changepoints at $\hat{t}_{a}, \hat{t}_{b}$ :

$$
\begin{aligned}
\hat{C}^{N S}\left(x_{0: n}, T, E^{\prime}, \beta\right) & =\hat{C}^{N S}\left(x_{0: a^{\prime}}, T, E^{\prime}, \beta\right)+C\left(p_{1} ; \hat{\theta}_{1}\right)+\min _{\theta \geq \hat{\theta}_{1}} C\left(p_{2} ; \theta\right)+2 \beta+\hat{C}^{N S}\left(x_{b^{\prime}: n}, T, E^{\prime}, \beta\right) \\
& =\hat{C}^{N S}\left(x_{0: a^{\prime}}, T, E^{\prime}, \beta\right)+C\left(p_{1} \cup p_{2} ; \hat{\theta}_{1}\right)+2 \beta+\hat{C}^{N S}\left(x_{b^{\prime}: n}, T, E^{\prime}, \beta\right) \\
& \geq \hat{C}^{N S}\left(x_{0: a^{\prime}}, T, E^{\prime}, \beta\right)+\min _{\theta} C\left(p_{1} \cup p_{2} ; \theta\right)+2 \beta+\hat{C}^{N S}\left(x_{b^{\prime}: n}, T, E^{\prime}, \beta\right) \\
& =\hat{C}^{N S}\left(x_{0: n}, T_{-p 2}, E^{\prime \prime}, \beta\right)+\beta,
\end{aligned}
$$

with some pairing $E^{\prime \prime}$. But since $T_{-p 2}$ is inconsistent, given $\mathscr{A}$ and $n>n_{0}$, we know that $\hat{C}^{N S}\left(x_{0: n}, T_{-p 2}, E^{\prime \prime}, \beta\right)>\hat{C}^{N S}\left(x_{0: n}, T, E, \beta\right)$.

We complete the proof by showing that $\hat{\theta}_{2} \geq \hat{\theta}_{1}$ is not permitted given $\mathscr{A}$. Monotonicity of $C$, together with Lemma 4 , means that given $\mathscr{A}$ and some $n>n_{0}$, there exists $K(n)$ such that $\hat{\theta}_{2} \in\left(\theta_{0}-K(n), \theta_{0}+K(n)\right)$. Recall that under these conditions, we also have consistency:

$$
\hat{C}^{c l}\left(x, T_{-p 2}, \beta\right)>\hat{C}^{c l}(x, T, \beta)
$$

Assume that $\left\{\hat{\theta}_{2}=\hat{\theta}_{1}\right\}$ is possible given $\mathscr{A}$. Then $\min _{\theta} C\left(p_{1} ; \theta\right)+\min _{\theta} C\left(p_{2} ; \theta\right)=$ $\min _{\theta} C\left(p_{1} \cup p_{2} ; \theta\right)$, so $\hat{C}^{c l}\left(x, T_{-p 2}, \beta\right)=\hat{C}^{c l}(x, T, \beta)-2 \beta<\hat{C}^{c l}(x, T, \beta)$, which is a contradiction. Therefore, $\left\{\hat{\theta}_{2}=\hat{\theta}_{1}\right\} \notin \mathscr{A}$, so $\hat{\theta}_{1} \geq \theta_{0}+K(n)$, and $\left\{\hat{\theta}_{2} \geq \hat{\theta}_{1}\right\} \Longrightarrow$ $\left\{\hat{\theta}_{2} \geq \theta_{0}+K(n)\right\}$, which is not possible given $\mathscr{A}$.

Lemma 7. Any $E^{\prime}$ where a non-background piece $p_{1}$ is classified as background will not be optimal:

$$
\hat{C}^{N S}\left(x_{0: n}, T, E^{\prime}, \beta\right)>\hat{C}^{N S}\left(x_{0: n}, T, E, \beta\right)
$$

Proof. Following the same approach as the previous Lemma.

The full Theorem can now be proven by induction. Assume the tuples in the minimum-cost segmentation $\hat{E}$ are ordered by the time of the smaller element (estimated $\hat{s}$ ) in each pair.

Take any $j$ which should be paired to some $j_{e}$, i.e. $\left(j, j_{e}\right) \in E$. Assume all pairs including points $0,1, \ldots, j-1$ have already been assigned correctly. Since $\operatorname{deg}(j)=$ 1 , there must be a pair $(j, t) \in \hat{E}$.

If $t<j$, the pair cannot be in $\hat{E}$, because then $\operatorname{deg}(t)>1$, violating Lemma 5 . Any $j<t<j_{e}$ is also not permitted, because then either $\operatorname{deg}(t)>1$, or $x_{t: t+1}$, which is not background, is classified as background and $\hat{E}$ cannot have the minimum cost 
by Lemma 7. Finally, $t>j_{e}$ is not permitted, because then $x_{j_{e}: j_{e}+1}$, which is background, is assigned to non-background and such $\hat{E}$ would not have the minimum cost by Lemma 6. Therefore, $j$ will be paired up correctly, $t=j_{e}$.

When $j=1$, all preceding points are assigned correctly (because 0 cannot be used by Lemma 5). By induction, all pairs in $\hat{E}$ will be correct, $\hat{E}=E$.

Segment type assignments also follow from consistency. For any true signal segment $s, e$, inaccurate assignment can be obtained if $|\hat{s}-\hat{e}|>l$. But for any consistent $\hat{T}, \hat{E}$ :

$$
|\hat{s}-\hat{e}|<\left|s+\mathscr{O}\left(\log (n)^{1+\delta}\right)-\left(e-\mathscr{O}\left(\log (n)^{1+\delta}\right)\right)\right|<l
$$

by definition of $l$. Similarly, for any nuisance segment $s_{N}, e_{N}$, given consistent segmentation and large $n,\left|\hat{s}_{N}-\hat{e}_{N}\right|>\left|s_{N}-e_{N}\right|-\mathscr{O}\left(\log (n)^{1+\delta}\right)>l$. Therefore, for sufficiently large $n$, any consistent $\hat{T}, \hat{E}$ will also have accurate segment type assignment.

Remark. The proof can be adapted to show that consistency also holds when $\theta_{0}$ is estimated with precision $\mathscr{O}(\sqrt{\log (n) / n})$; see Lemma 9 in Fisch et al. (2018).

\section{Overestimation of segment length}

Lemma 8 ((Fisch et al., 2018)). There exists a constant $A_{2}(\alpha)$, such that for any interval $x_{a: b}$ that contains a single changepoint $t_{i}$ and satisfies:

$$
b-t_{i} \geq t_{i}-a \geq \frac{A_{2}(\alpha)}{\min \left(\Delta_{i}, \Delta_{i}^{2}\right)} \log (n)^{1+\delta}
$$

the cost of missing the changepoint satisfies the following:

$$
\hat{C}\left(x_{a: b}\right)-\hat{C}\left(x_{a: t_{i}}\right)-\hat{C}\left(x_{t_{i}: b}\right) \geq \alpha \log (n)^{1+\delta} .
$$

Theorem 3. Assume the same set-up as in Theorem 2, but let the segment length be overestimated: $l^{\prime} \geq \min \left|s_{j}^{N}-e_{j}^{N}\right|-\log (n)^{1+\delta_{2}}>l \geq \max \left|s_{i}-e_{i}\right|+\log (n)^{1+\delta_{2}}$. By minimising $\hat{C}^{N S}$, all non-nuisance changepoints will still be estimated consistently:

$$
P\left(\forall s_{i}, e_{i}, \exists \hat{s}_{i}, \hat{e}_{i}: \max \left(\left|\hat{s}_{i}-s_{i}\right|,\left|\hat{e}_{i}-e_{i}\right|\right)<\frac{A_{1}}{\min \left(\Delta_{i}, \Delta_{i}^{2}\right)} \log (n)^{1+\delta}\right) \geq 1-B n^{-\epsilon},
$$

for some constants $A_{1}, B, \epsilon, n>n_{1}$. 
Proof. Consider $x_{i: j}$ such that $x_{i}, x_{j}$ are true background points. By additivity of $C^{N S}$, consistency over such blocks will imply consistency over $x_{0: n}$. Let $\mathscr{T}_{l}$ be the set of all segmentations $T$ permissible when the segment length parameter is set to $l$. Denote by $T^{0}$ the true segmentation, and by $T^{\prime}$ a segmentation that is inconsistent for a segment, i.e., there exists a true signal segment $s, e \in T^{0}, i<s<j<e$ for which:

$$
\forall s \text { or } e \in T^{\prime}:|\hat{s}-s| \geq \frac{A_{1}}{\min \left(\Delta_{i}, \Delta_{i}^{2}\right)} \log (n)^{1+\delta} \text { or }|\hat{e}-e| \geq \frac{A_{1}}{\min \left(\Delta_{i}, \Delta_{i}^{2}\right)} \log (n)^{1+\delta} .
$$

We will show that there always exists a consistent solution $T$ :

$$
\begin{gathered}
T \in \mathscr{T}_{l^{\prime}} \\
\forall T^{\prime} \in \mathscr{T}_{l^{\prime}}, C(T)<C\left(T^{\prime}\right) .
\end{gathered}
$$

Case 1. When there are no nuisance segments $s_{N}, e_{N}$ such that $i<s_{N}<s<e<$ $e_{N}<j,\left|s_{N}-e_{N}\right|<l^{\prime}$. (This covers any cases where signal and nuisance segments do not overlap, or the nuisance segments are long enough to be classified correctly.) Then $T=T^{0}, E=E^{0}$ satisfies (S20). Since any $T^{\prime}$ must have $\left|T^{\prime}\right| \neq\left|T^{0}\right|$, or inconsistent estimation of at least one changepoint, (S21) follows from consistency.

Case 2. When there exists a nuisance segment with overlapping signal segment(s), and $\left|s_{N}-e_{N}\right|<l^{\prime}$. Consider inconsistent segmentations of the type $T^{\prime}=$ $\left\{\left(s_{N}, e_{N}^{\prime}\right),\left(s, e^{\prime}\right)\right\}$ with $e^{\prime}>e+\frac{A_{2}(2 \alpha)}{\min \left(\Delta_{i}, \Delta_{i}^{2}\right)} \log (n)^{1+\delta}$, and $e_{N}^{\prime}-s_{N}>l^{\prime}$. Choose $T^{0^{\prime}}=$ $\left\{\left(s_{N}, e_{N}^{\prime}\right),(s, e),\left(e, e^{\prime}\right)\right\}$, which satisfies (S20). The second condition is satisfied by applying Lemma 8 with $A_{2}(2 \alpha)$ :

$$
\hat{C}^{N S}\left(x_{i: j}, T^{\prime}, \beta\right)-\hat{C}^{N S}\left(x_{i: j}, T^{0^{\prime}}, \beta\right)=\hat{C}\left(x_{s: e^{\prime}}\right)-\hat{C}\left(x_{s: e}\right)-\hat{C}\left(x_{e: e^{\prime}}\right)-\beta>2 \beta-\beta>0
$$

When $e^{\prime}<e-\frac{A_{2}(2 \alpha)}{\min \left(\Delta_{i}, \Delta_{i}^{2}\right)} \log (n)^{1+\delta}$, we can apply a similar argument, with $T^{0^{\prime}}=$ $\left\{\left(s_{N}, e_{N}^{\prime}\right),(s, e)\right\}$ :

$$
\begin{aligned}
\hat{C}^{N S}\left(x_{i: j}, T^{\prime}, \beta\right)-\hat{C}^{N S}\left(x_{i: j}, T^{0^{\prime}}, \beta\right) & =\hat{C}\left(x_{s_{N}: s \cup e^{\prime}: e_{N}}\right)+\hat{C}\left(x_{s: e^{\prime}}\right)-\hat{C}\left(x_{s_{N}: s \cup e: e_{N}}\right)-\hat{C}\left(x_{s: e}\right) \\
& >\hat{C}\left(x_{s: e^{\prime}}\right)+\hat{C}\left(x_{e^{\prime}: e}\right)-\hat{C}\left(x_{s: e}\right)+\beta \\
& >0
\end{aligned}
$$

The case for inconsistent $s^{\prime}$ is identical. Similarly, using previous consistency results, it is easy to show that $\hat{C}^{N S}\left(x_{i: j}, T^{\prime}, \beta\right)$ for any $T^{\prime}:\left|T^{\prime}\right| \neq|T|$ is not minimal. 
Remark. When there exists a nuisance segment $s_{N}, e_{N}: i<s_{N}<e_{N}<j, \mid s_{N}-$ $e_{N} \mid<l^{\prime}$, but no signal segments overlap it (no $s, e: s_{N}<s<e<e_{N}$ ). Choose $T$ that has the same changepoint positions as $T^{0}$, but any segment $\left|s_{N}-e_{N}\right|<\hat{l}$ is assigned to the segment type. This satisfies (S20), and since $C(T)=C\left(T^{0}\right)$, (S21) holds. In other words, such short nuisance segments are still detected, but appear as false positives (signal segments) when the length cutoff is overestimated.

Remark. The standard epidemic segmentation without overlapping segments $T^{E}=\left\{\left(s_{N}, s_{1}\right),\left(s_{1}, e_{1}\right), \ldots,\left(e_{k}, e_{N}\right)\right\}$ is always in $\mathscr{T}_{l^{\prime}}$. When $l^{\prime}>>\left|e_{N}-s_{N}\right|$, any overlapping segmentation incurs a high inconsistency penalty, and $T^{E}$ becomes the optimal segmentation. This shows that, as expected, with $l^{\prime}=n$ our method reduces to the standard epidemic detector. 


\section{Nuisance-robust segmentation algorithm}

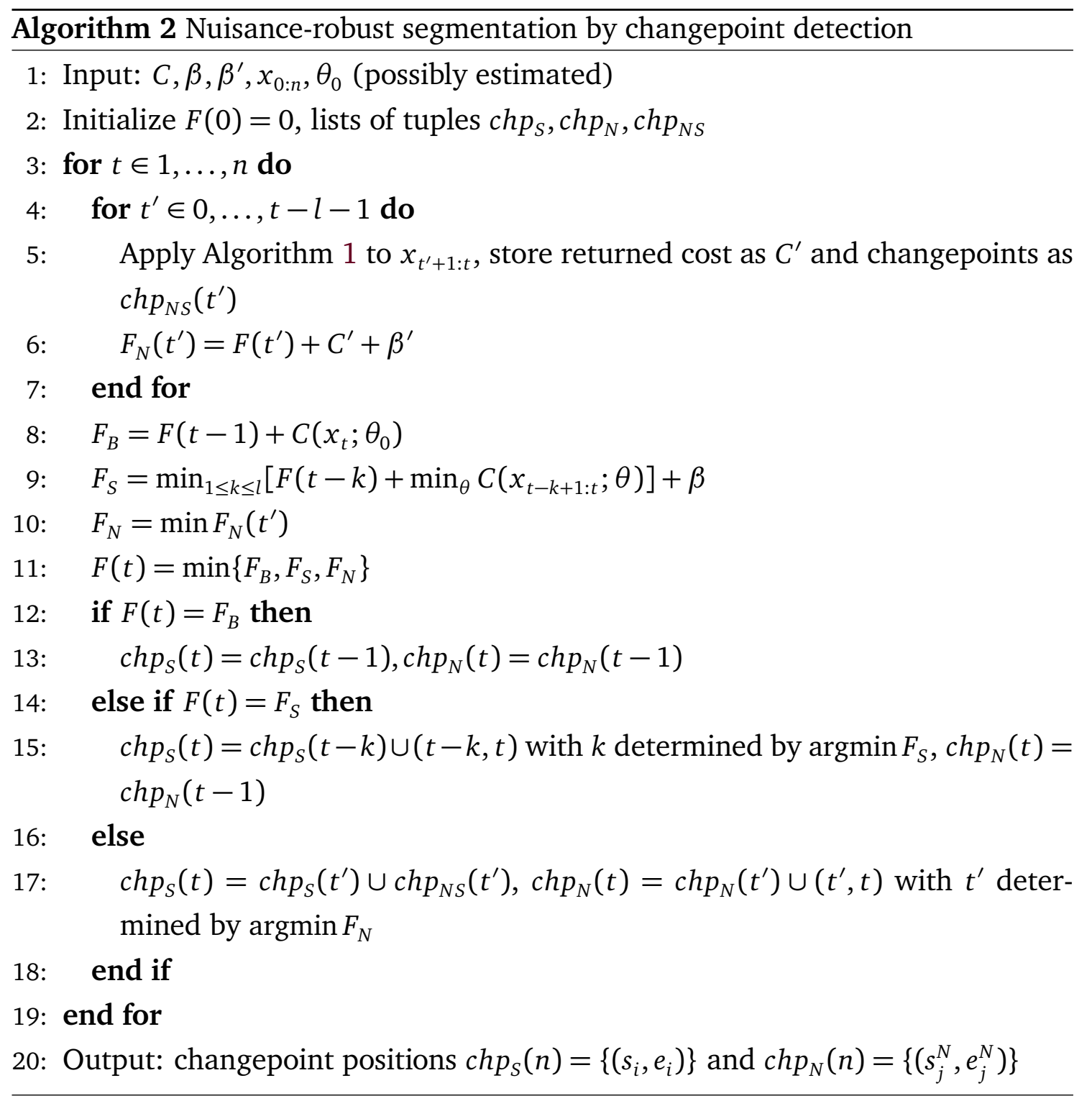




\section{E Supplementary figures}

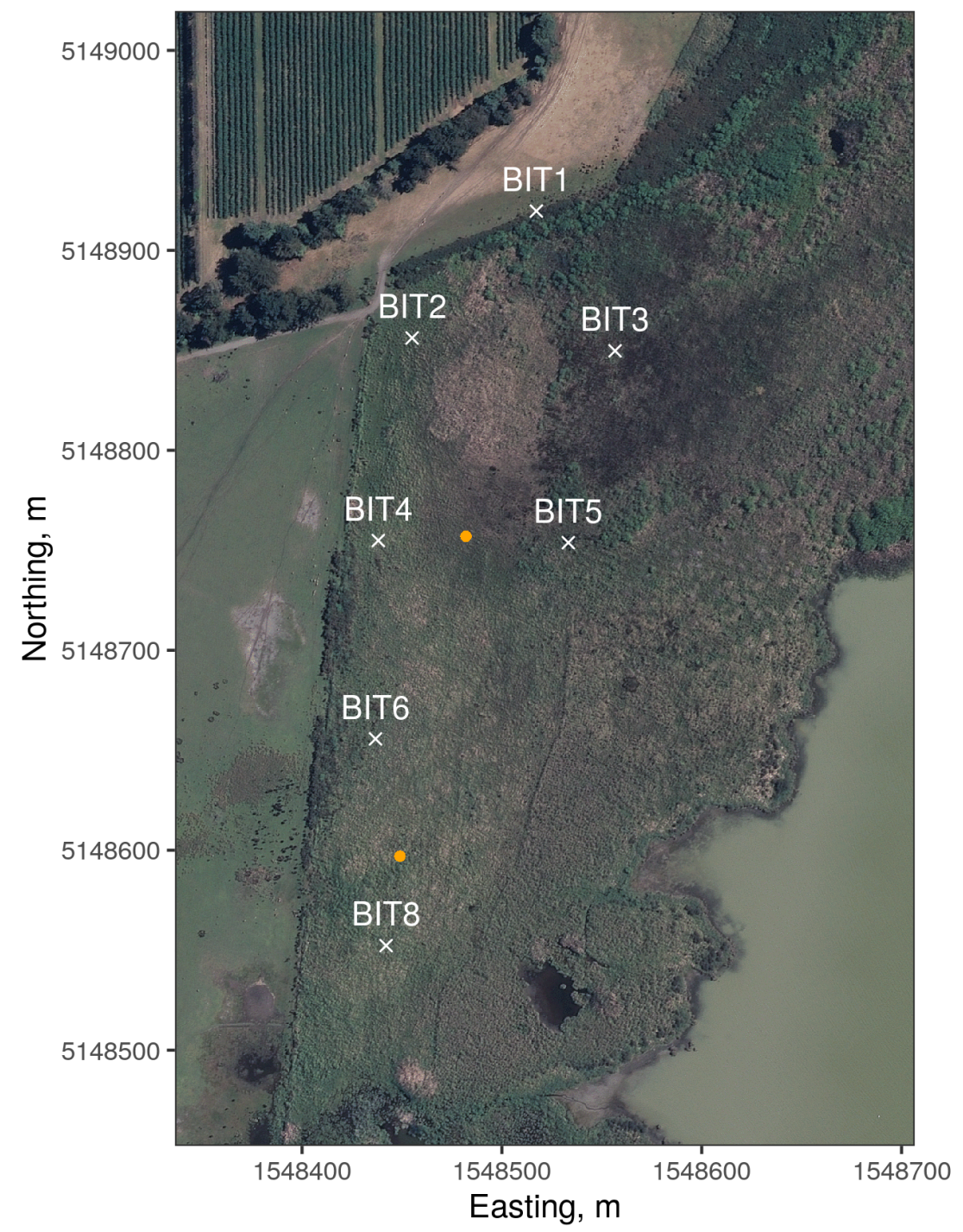

Figure S3: Map of bittern survey area near Lake Ellesmere, New Zealand. Crosses: positions of recorders, orange dots: positions of playback speakers. Aerial photography from LINZ Data Service, projection NZTM2000. 


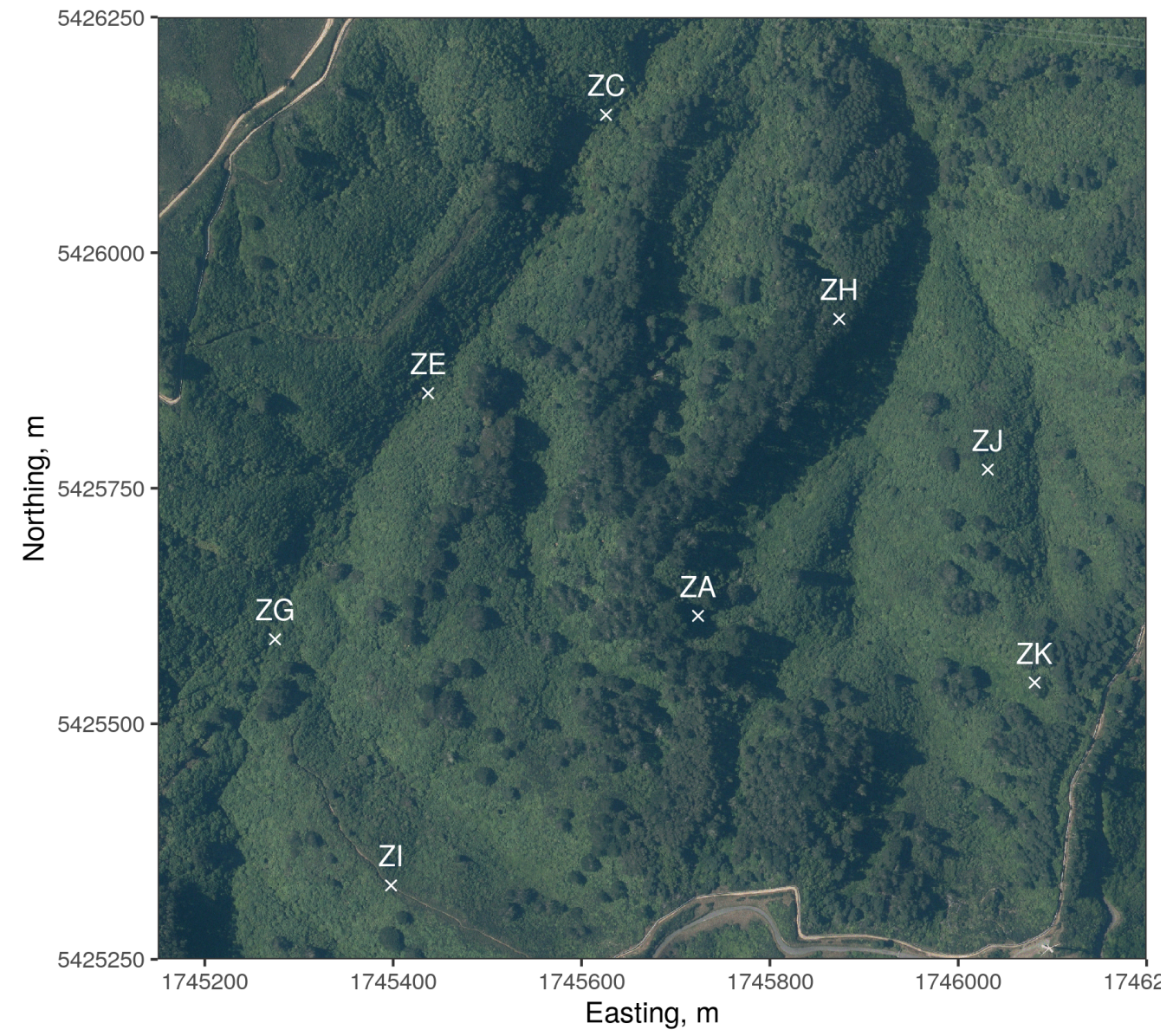

Figure S4: Map of little spotted kiwi survey area in Zealandia sanctuary, Wellington, New Zealand. Crosses: positions of recorders. Aerial photography from LINZ Data Service, projection NZTM2000. 


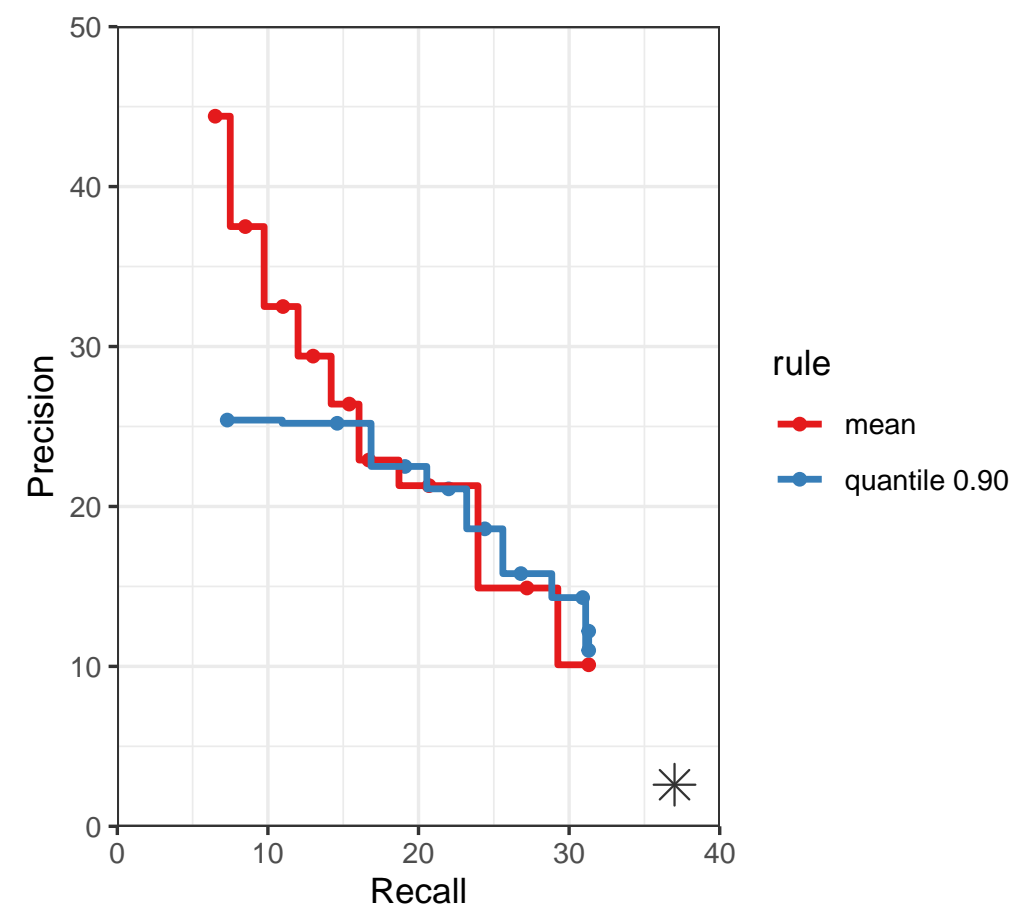

Figure S5: Precision-recall curve for the changepoint detector with MFCC classifier, obtained on DCASE data. Two different rules were tested to aggregate per-frame classifier output into segment-level decisions: mean exceeding a threshold, or 90th percentile exceeding a threshold. Each point corresponds to a different value of the corresponding threshold. The star marks the precision and recall values obtained with changepoint detector only (no subsequent classifier).

\section{Bibliography}

Bottou, L. (1998) Online algorithms and stochastic approximations. In Online Learning and Neural Networks (ed. D. Saad). Cambridge, UK: Cambridge University Press. Revised Oct 2012.

Fisch, A. T. M., Eckley, I. A. and Fearnhead, P. (2018) A linear time method for the detection of point and collective anomalies. arXiv preprint: 1806.01947.

Juodakis, J. and Marsland, S. (2020) Epidemic changepoint detection in the presence of nuisance changes. arXiv preprint: 2008.08240 .

Marchand, E. (1996) Computing the moments of a truncated noncentral chi-square distribution. Journal of Statistical Computation and Simulation, 54, 387-391. 


\title{
Wind-robust sound event detection and denoising for bioacoustics
}

\author{
Julius Juodakis ${ }^{1}$, Stephen Marsland
}

Manuscript submitted for publication

\section{Abstract}

1. Sound recordings are used in various ecological studies, including wildlife monitoring by acoustic surveys. Such surveys require automatic detection of target sound events in the large amount of data produced. However, current detectors, especially those relying on band-limited energy as the main feature, are severely impacted by wind, which causes transient energy increases. The rapid dynamics of this noise invalidate standard noise estimators, and no satisfactory method for dealing with it exists in bioacoustics, where simple training and generalization between conditions are important.

2. We propose to estimate the transient noise level by fitting short-term spectrum models to a wavelet packet representation. This estimator is then combined with log-spectral subtraction to stabilize the background level. The resulting adjusted wavelet series can be analysed by standard energy detectors. We use real data from long-term monitoring to tune this workflow, and test it on two acoustic surveys of birds. Additionally, we show how the estimator can be incorporated in a denoising method to restore sound.

3. The proposed noise-robust workflow greatly reduced the number of false alarms in the surveys, compared to unadjusted energy detection. As a result, the acoustic survey efficiency (precision of the estimated call density)

\footnotetext{
${ }^{1}$ Corresponding author. Email: julius.juodakis@sms.vuw.ac.nz. J. Juodakis and S. Marsland at School of Mathematics and Statistics, Victoria University of Wellington, New Zealand.
} 
improved for both species. Denoising was also more effective when using the short-term estimate, whereas standard wavelet shrinkage with a constant noise estimate struggled to remove the effects of wind.

4. In contrast to existing methods, the proposed estimator can adjust for transient broadband noises without requiring additional hardware or extensive tuning to each species. It improved the detection workflow based on very little training data, making it particularly attractive for detection of rare species.

\section{Introduction}

In recent years, acoustic surveys based on long-term recordings have emerged as a powerful tool in ecology. Such surveys can cover large scales in both time and space, making them invaluable for monitoring animal species in conservation and behaviour research (see reviews by Shonfield and Bayne (2017); Sugai et al. (2018)). Many further applications for such monitoring at the human-wildlife interface have been proposed, such as poaching detectors (Astaras et al., 2017), warning systems for elephant approach (Zeppelzauer et al., 2015), or farm animal welfare monitoring (Mcloughlin et al., 2019).

A key step in most of these tasks is the detection of target sounds in the recordings. The resulting annotations can then be used in various inference models, population size estimation (Dawson and Efford, 2009), source localization (Rhinehart et al., 2020), or for other purposes. Since the amounts of data recorded often total in the thousands of hours, and calls are intermittent within them, automatic detection is necessary, and choosing the right methods can have a large impact on survey efficiency (Juodakis et al., 2021a). Thus, developing detectors that can be applied to natural soundscapes is an important and active area of research.

A major obstacle for current bioacoustic sound detectors is environmental noise, in particular wind (Priyadarshani et al., 2018). Wind interaction with microphones creates noise in the form of transient peaks, with higher power in lower frequencies (Walker and Hedlin, 2009; Nelke, 2016). Detection in bioacoustics, at least in initial stages, typically identifies sound events as increases in energy, possibly band-filtered (e.g., Prince et al. (2019)), transformed (Priyadarshani et al., 2020) or in the spectrogram representation (Lasseck, 2013). Wind peaks can appear as such increases, and therefore create false positives, thus greatly reducing the detection performance. More complex recognisers are also impaired by wind: Digby et al. (2013) used a decision tree based on handcrafted species-specific features that 
performed considerably worse in windy conditions, while Znidersic et al. (2021) observed similar issues when estimating call counts based on acoustic indices of 1minute frames. While the exact mechanism of this effect is not clear, rapid changes in background energy and degradation of target sound features are likely causes, and methods robust to these factors are needed to allow detection in natural conditions.

Various approaches to wind noise suppression have been developed for different tasks. Classic denoising methods such as the Wiener or MMSE filters are not applicable to wind because of its rapid dynamics. Neural networks have been successfully used for speech denoising, e.g., Keshavarzi et al. (2018), and in public competitions (Kahl et al., 2019). However, their adoption in bioacoustic practice has been limited, primarily because they require large quantities of training data, which is rarely available for wildlife. In addition, the black box nature of deep learning makes it unclear if such models would generalize to different surveys, as similar geophonic noise sources in different areas can have different noise profiles (Metcalf et al., 2020). For example, in a recent study Vickers et al. (2021) observed that denoising by neural networks does not help subsequent call detection with unseen types of noise. Subsequent ecological inference often also makes some assumptions about the detection probability (e.g., smooth decrease with distance, Dawson and Efford (2009)) that are difficult to verify with such methods, so more transparent wind-robust detectors are needed.

Some simpler methods for wind denoising have been developed in other fields, but are not applicable to bioacoustics. For example, the signal centroids method (Nelke et al., 2014) relies on the target having high dominant frequency, which is simply not true for many vocalizing species. Other methods require pitch estimation (Nelke and Vary, 2015), which is itself a complex task for distant and noisy sounds in natural environments. Another distinct research area is noise mitigation by shielding, mechanical integration, or multi-microphone coherence (Walker and Hedlin, 2009). We will not consider these options in this study, as they require physical modifications to hardware, complicate recorder deployment, and do not help analyse historical or ongoing survey data.

Therefore, we propose a new procedure for single-microphone estimation of transient broadband noise. We use it to improve the noise-robustness of an acoustic event detection method. We will first describe the theoretical basis of this method, and then demonstrate its usage on two surveys of birds. We also show how this estimate can be incorporated in a denoising method to restore clean sound for listening 
or visualization. The proposed noise estimator is found to considerably improve the efficiency of acoustic surveys, and is easily adaptable to different species and noise profiles.

\section{Materials and methods}

\subsection{Overview of the proposed detector}

The main method proposed in this paper is a wind-robust energy detector. It detects signals in a target frequency band using these steps:

1. Sound is converted to a wavelet packet tree (WPT) representation, and node(s) corresponding to the target frequencies are chosen;

2. The noise level in the chosen node is interpolated using a log-log line, fitted to the energies in non-target nodes by least-squares or quantile regression;

3. The estimated noise level is used in log-scale spectral subtraction to adjust the target band energy;

4. Adjusted energies are analysed by a changepoint detection algorithm, presented previously (Juodakis et al., 2021b), to detect increases, which are assumed to be calls.

We will now present each of these components in more detail, starting with the final detection stage which is used to guide the other parts of the method.

\subsection{Energy-based signal detection}

In the energy detection framework, the sequence of observations $Y_{t}$ is modelled as the sum of a stationary noise process $N_{t}$ and signal $S_{t}$ :

$$
Y_{t}=N_{t}+S_{t}
$$

The signal is transient, and its presence is detected based on the observed energy $y_{t}^{2}$. This is motivated by assuming that both $N, S$ are independent white Gaussian processes:

$$
N_{t} \sim \mathscr{N}\left(0, \sigma_{N}^{2}\right), S_{t} \sim \mathscr{N}\left(0, \sigma_{S}^{2}(t)\right)
$$


Then, testing $y_{t}^{2}$ against a fixed threshold is a generalized likelihood ratio test for the hypotheses:

$$
\begin{aligned}
& H_{0}: \sigma_{S}^{2}(t)=0 \\
& H_{1}: \sigma_{S}^{2}(t)>0
\end{aligned}
$$

and its properties, such as error rates, can be determined theoretically (Chen, 2010). For example, false alarm rates can be controlled at rate $\alpha$ by setting the threshold to $\lambda=\sigma_{N}^{2} F^{-1}(1-\alpha)$, where $F$ is the CDF of $\chi_{1}^{2}$ distribution, and $\sigma_{N}^{2}$ is estimated utilising the stationarity assumption, e.g., from quiet frames. Larger intervals $[s, e]$ can be tested using windowed statistics such as $\sum_{i=s}^{e} y_{i}^{2}$ or $\max _{i \in[s, e]} y_{i}^{2}$. The energy can also be compared at many positions, to locate the start and end of signal activity (changepoint detection; Page (1954)). We will use a variation of this changepoint procedure, presented in Juodakis et al. (2021b), as the main detector in this study, but our results apply to any method that uses the energy $Y_{t}^{2}$ for detection.

In all the above methods, the stationary noise model (2) is key to detection. Wind, and transient broadband noises in general, violate this assumption and harm the performance. For example, if the noise is Gaussian with transient increases in power:

$$
Y_{t}=N_{t}+S_{t}, N_{t} \sim \mathscr{N}\left(0, \sigma_{N}^{2}+\sigma^{2}(t)\right),
$$

then the test $y_{t}^{2} \lessgtr \sigma_{N}^{2} F^{-1}(1-\alpha)$, established on quiet periods as before, will have a false alarm rate greater than $\alpha$. In fact, without assuming any further features distinguishing the wind and signal processes, the situations $Y_{t}=S_{t}$ and $Y_{t}=N_{t}$ are not identifiable: this can be seen in an example of a (band-limited) energy series where both a bird call and a wind gust correspond to a transient increase (Figure 1). Therefore, no conclusions about the performance of energy detection under these conditions can be made, in contrast to the stationary background model (1)-(2).

\subsection{Wavelet packet representation}

Energy detection is typically applied to a band-limited representation of the recorded sound, in order to reduce the impact of non-target signals. In this paper, we will use sound frequency subbands obtained from a wavelet packet tree (WPT). Bioacoustic detectors using WPT have been used previously (Zhang and Li, 2015; Priyadarshani et al., 2020; Juodakis et al., 2021b). It is a multiscale decomposition, defined at each scale $j=1, \ldots, J$ by a set of orthogonal filters $\left\{\psi_{j, k}\right\}_{k \in 1, \ldots, 2^{j}}$, which approxi- 

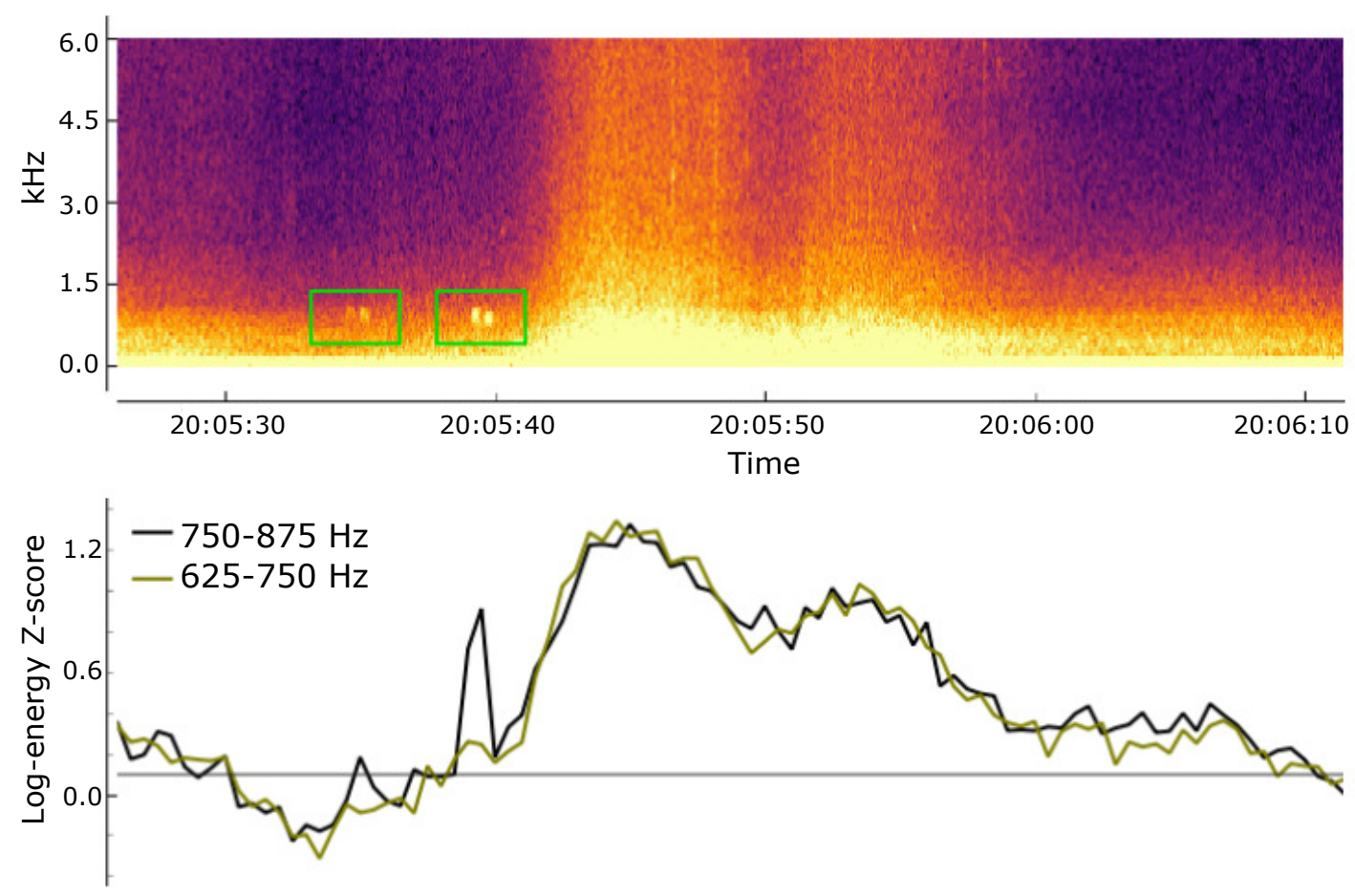

Figure 1: Top: a spectrogram showing two calls of a morepork (New Zealand owl, Ninox novaeseelandiae) surrounded by wind noise. Bottom: wavelet coefficient log-energy for two frequency bands, extracted from the same clip in $0.5 \mathrm{~s}$ windows and standardized. The target band (black) shows two energy peaks corresponding to the calls, but a threshold that would capture these peaks (marked in grey) is also exceeded by the wind gust seen here. However, the wind energies in this band and a non-target band (green) appear correlated, suggesting that they could be estimated by an appropriate interpolation across frequencies.

mately bandpass the signal to the frequency range $\left[F_{N}(k-1) / 2^{j}, F_{N} k / 2^{j}\right]$. Here $F_{N}$ is the Nyquist frequency, equal to half of the sampling rate. Denoting by $X_{t}$ the original sound waveform, each node $(j, k)$ of the WPT thus contains the series of coefficients:

$$
Y_{j, k, t}=\sum_{i=1}^{n} \psi_{j, k}(t-i) X_{i}
$$

obtained by applying the filter $\psi_{j, k}$.

Before application, the user selects a node $\left(j_{0}, k_{0}\right)$ likely to contain the target signal, i.e., the signal is detected in $Y_{t}:=Y_{j_{0}, k_{0}, t}$. The choice can be made a priori based on the expected frequency range of the target, or by a training process, for example as described in Priyadarshani et al. (2020). (We will assume that a single target node is chosen for simplicity of notation, but the method proposed here directly applies with several nodes as well.)

In addition to removing out-of-band interference, the wavelet transform decor- 
relates various types of noise (Wornell, 1993), thus allowing methods derived under white Gaussian noise assumptions to be applied to more diverse problems. Increases in energy, caused by either in-band signal or transient broadband noises, are preserved by this transform (Figure 1).

\subsection{Interpolating noise level}

To allow detection in windy conditions, we propose a new method for estimating the level of transient broadband noise. The main idea is, at each time point, to fit a regression line to subband energies, and use that to interpolate the energy in the target band.

We will assume for now that the following model consisting of only wind and target signal, and later discuss relaxing it to allow other signals and noises.

Assumption 1. For any WPT node $j, k$ :

$$
Y_{j, k, t}= \begin{cases}N_{j, k, t}+S_{j, k, t} & \text { if } k=k_{0}(\text { target node) } \\ N_{j, k, t} & \text { otherwise }\end{cases}
$$

where $N_{j, k, t}$ and $S_{j, k, t}$ are the wavelet-transformed wind noise and signal components, respectively.

We wish to estimate $N_{j, k_{0}, t}$ (or the noise power $\left|N_{j, k_{0}, t}\right|^{2}$ ), while for all other bands the $N_{j, k, t}=Y_{j, k, t}$ are directly observed.

We start with the following formula of short-term wind power spectral density, which was derived from fluid dynamics and has since been verified empirically (Walker and Hedlin, 2009; Nelke, 2016):

$$
P S D(f, t)=c(t) / f^{\alpha},
$$

where $f$ is the frequency, $\alpha$ a constant, and $c(t)$ a factor that depends on the wind strength at time $t$ and microphone properties. This is an example of the $1 / f$ class of processes, for which decorrelation by wavelet transform has been extensively studied: the resulting wavelet coefficients are almost uncorrelated within and between nodes (Wornell, 1993). In addition, their distribution is Gaussian if the original $1 / f$ process is Gaussian, also known as fractional Brownian noise (proven trivially, by linearity of the transform), or for other processes converges to Gaussian with sufficiently large $j$ (Atto and Pastor, 2010), or by averaging multiple coefficients (Serroukh et al., 2000). 
Thus, we will continue assuming that at each time point, the wind WPT coefficients vector $\mathbf{N}_{j, t}=\left[N_{j, 1, t}, \ldots, N_{j, 2^{j}, t}\right]$ is multivariate Gaussian:

$$
\mathbf{N}_{j, t} \sim \mathscr{N}\left(0, \operatorname{diag}\left(\sigma_{j, 1}^{2}(t), \ldots, \sigma_{j, 2^{j}}^{2}(t)\right)\right)
$$

The variance of the coefficients is close to the PSD at each node's centre frequency $f_{j, k}=F_{N}(k-1 / 2) / 2^{j}$ (Atto et al., 2010; Moulines et al., 2007):

$$
\sigma_{j, k}^{2}(t) \approx P S D\left(f_{j, k}, t\right)=c(t) / f_{j, k}^{\alpha}
$$

Their energy is the square of a Gaussian and thus $\chi^{2}$-distributed:

$$
\left|N_{j, k, t}\right|^{2} \sim c(t) f_{j, k}^{-\alpha} \chi_{1}^{2} \Rightarrow \log \left|N_{j, k, t}\right|^{2} \sim-\alpha \log f_{j, k}+\log c(t)+\log \chi_{1}^{2}
$$

In other words, there is a linear relationship between $\log \left|N_{j, k, t}\right|^{2}$ and $\log f_{j, k}$. This suggests that the target band noise level $\log \left|N_{j, k_{0}, t}\right|$ could be interpolated using an OLS regression of $\mathbf{x}_{k}=\left[1, \log f_{j, k}\right]$ and $\mathbf{y}_{k}=\log \left|Y_{j, k, t}\right|^{2}$ :

$$
\hat{N}^{O L S}:=\mathbf{x}_{k_{0}} \hat{\beta}, \hat{\beta}=\underset{\beta}{\operatorname{argmin}} \sum_{k \neq k_{0}}\left(\mathbf{y}_{k}-\mathbf{x}_{k} \beta\right)^{2} .
$$

Furthermore, we can use the properties $\mathbb{E} \log \chi_{n}^{2}=\psi(n / 2)+\log 2$, where $\psi$ is the digamma function, and $\operatorname{Var}\left(\log \chi_{n}^{2}\right)=\zeta(2, n / 2)$ with $\zeta$ being the generalized Riemann (Hurwitz) zeta function (Veitch and Abry, 1999), to obtain:

$$
\begin{aligned}
\mathbb{E} \log \left|N_{j, k, t}\right|^{2} & =-\alpha \log f_{j, k}+\log c(t)+\psi(1 / 2)+\log 2 \\
\operatorname{Var}\left(\log \left|N_{j, k, t}\right|^{2}\right) & =\zeta(2,1 / 2)
\end{aligned}
$$

In other words, the error term is homoscedastic, so standard OLS results can be applied to show the estimation properties. In particular, we have that, under Assumption 1 and wind model (4), it is consistent: $\hat{N}^{O L S} \stackrel{p}{\rightarrow} \mathbb{E} \log \left|N_{j, k_{0}, t}\right|^{2}$.

Note also that instead of individual coefficients, short-term sums $\log \sum_{i=s}^{e}\left|Y_{j, k, i}\right|^{2}$ could be used as the $\mathbf{y}_{k}$ in the regression. If the wind strength factor $c(t)$ remains the same over this window, repeating the analysis above shows that the only change is smaller variance of the error term, as $\zeta(2,(e-s) / 2)$ decreases for longer windows.

Relaxing Assumption 1. While the $1 / f$ model covers a variety of noise processes, noise spectra obtained in field conditions may deviate from this model, due to frequency response of the microphone, shielding, and the recording device. 
Other noises may also be present. For example, white noise can be captured by the model as the special case with $\alpha=0$, but if both white and wind noise are present at comparable power, the resulting spectrum will no longer be $1 / f$. To allow adaptation to these issues, we propose including higher polynomial degrees of $\log f_{j, k}$ in the regression (5). We investigate possible choices of the polynomial degree on a pilot dataset in a later section.

Alternatively, Achard and Coeurjolly (2010) proposed some estimators designed to specifically reduce bias for estimation of contaminated $1 / f$ processes. However, these estimators only outperform the basic $\hat{N}^{O L S}$ if the contamination model is specified correctly, and even then require sufficient sample size, so we do not explore them further here.

Further, other narrow-band signals may be present, causing local deviations from the $N_{j, k, t}$ model. We then model the noise power as a contaminated distribution

$$
\left|N_{j, k, t}\right|^{2} \sim\left\{\begin{array}{l}
c(t) f_{j, k}^{-\alpha} \chi_{1}^{2} \text { with probability } 1-\epsilon \\
Z_{j, k, t} \text { with probability } \epsilon
\end{array}\right.
$$

where $Z_{j, k, t}$ is a random variable representing the power of contaminating signal and noise, and $\epsilon$ is the rate of contamination. If the contamination is sufficiently loud, so most of the mass of $Z_{j, k}$, is above $c(t) f_{j, k}^{-\alpha}$, the distribution of $\left|N_{j, k, t}\right|^{2}$ can be highly asymmetric, and thus estimation by OLS is significantly biased, especially if higher order terms are included.

An intuitive solution is to replace the square loss (5) used in regression with an asymmetric loss function, such as:

$$
L_{\tau}(\beta)=\sum_{k: k \neq k_{0}, \mathbf{y}_{k} \geq \mathbf{x}_{k} \beta} \tau\left|\mathbf{y}_{k}-\mathbf{x}_{k} \beta\right|+\sum_{k: k \neq k_{0}, \mathbf{y}_{k}<\mathbf{x}_{k} \beta}(1-\tau)\left|\mathbf{y}_{k}-\mathbf{x}_{k} \beta\right|
$$

and then estimate the noise level by:

$$
\hat{N}^{Q, \tau}:=\mathbf{x}_{k_{0}} \hat{\beta}, \hat{\beta}=\underset{\beta}{\operatorname{argmin}} L_{\tau}(\beta) .
$$

This procedure is in fact identical to quantile regression (QR), so $\hat{N}^{Q, \tau}$ estimates the $\tau$-quantile of the (contaminated) noise level given $\mathbf{x}_{k_{0}}$ (Koenker and Bassett, 1978). This allows us to use the known properties of quantile regression to analyse this estimator.

It can be shown that if sufficiently low quantile $\tau$ is chosen, then $\hat{N}^{Q, \tau}$ estimates the $\tau /(1-\epsilon)$-quantile of the uncontaminated noise distribution (Supplementary 
Material A). Thus the interpolation of the noise level is biased, but a bias adjustment factor can be obtained if $\hat{\epsilon}$ is available. Alternatively, note that by averaging the energy statistic over many windows, arbitrary variance reduction of the noise can be achieved, with all quantiles approaching $\mathbb{E}\left(\log \left|N_{j, k, t}\right|^{2}\right)$, and thus $\hat{N}^{Q, \tau}$ can be made consistent.

\subsection{Proposed adjustment method: log spectral subtraction}

Once an estimate of the noise level is obtained, it can be used to estimate the clean signal $\tilde{Y}_{t}$ from $Y_{t}$. A common method for this is spectral subtraction (Vaseghi, 2009), stated in its power form as:

$$
\left|\tilde{Y}_{t}^{2}\right|=\max \left(0,\left|Y_{t}^{2}\right|-\left|\hat{N}_{t}^{2}\right|\right)
$$

where $\hat{N}_{t}$ is an estimate of $N_{t}$. This results in strong suppression in low SNR conditions, which is desirable for many applications.

However, this adjustment does not work well for signal detection. So far, we have assumed that the noise power $N_{t}^{2}$ is a $\chi^{2}$-distributed random variable, and interpolation can at best provide some estimate of its expectation $\left|\hat{N}_{t}^{2}\right|=\sigma_{j, k}^{2}(t)$. The distribution of $\tilde{Y}_{t}^{2}$, produced by spectral subtraction, will then be a left-censored and shifted $\chi^{2}$ distribution (Figure 2A-B). Furthermore, we show that this adjusted distribution will still depend on $\sigma_{j, k}^{2}(t)$ (Supplementary Material B). Both of these issues violate the stationary Gaussian model (2), and so energy-based detectors applied after spectral subtraction even with perfect estimates will still not have the expected performance.

We propose that spectral subtraction for detection should be carried out on log scale, or equivalently:

$$
\left|\tilde{Y}_{t}^{2}\right|=\max \left(1,\left|Y_{t}^{2}\right| /\left|\hat{N}_{t}^{2}\right|\right)
$$

In contrast to (9), this log spectral subtraction will produce $\tilde{Y}_{t}^{2}$ distributed as $\chi_{1}^{2}$ (Figure 2C). Given an accurate estimate $\left|\hat{N}_{t}^{2}\right|=\sigma_{j, k}^{2}(t)$, the adjusted estimate will match model (2) with $\sigma_{N}^{2}=1$, and optimal performance of the detection methods derived under this model can be expected.

\subsection{Validating the noise spectrum fit on pilot data}

To investigate whether field recording data matches the $1 / f$ spectrum profile, we conducted a pilot experiment on a set of short clips randomly selected from a larger 

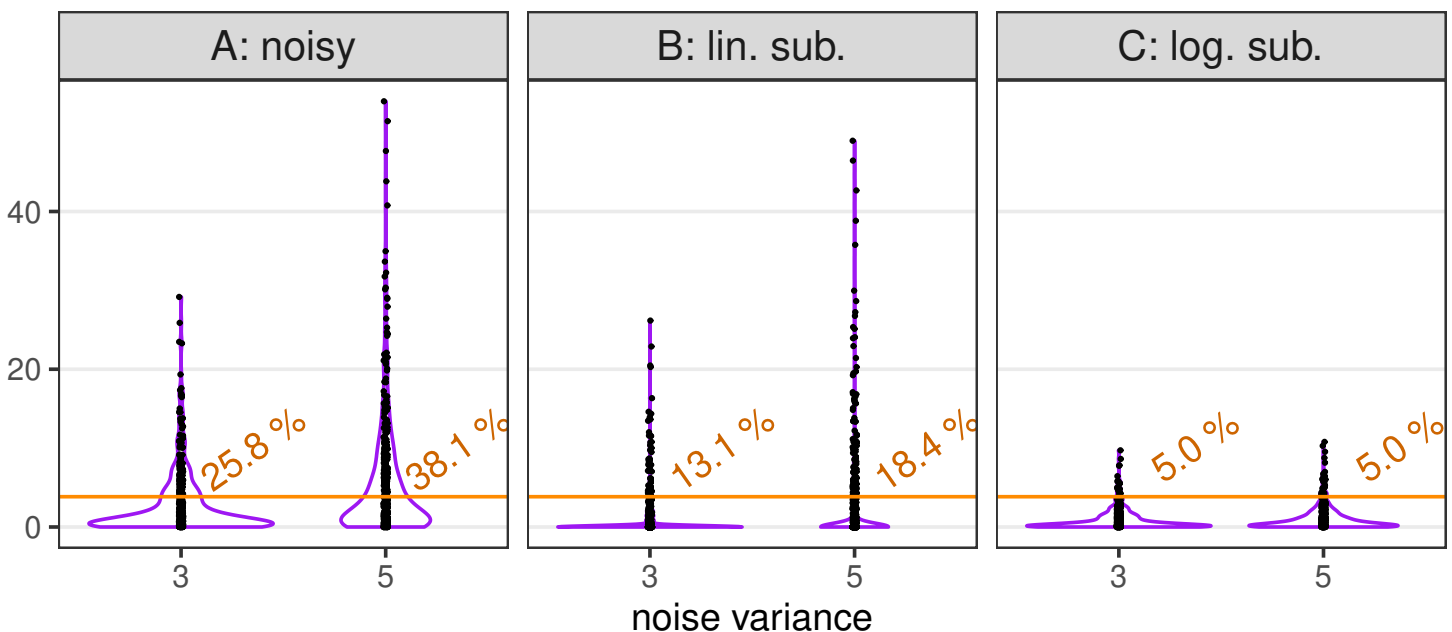

Figure 2: Violin plots showing the distribution of energy of: (A) Gaussian noise with $\sigma^{2}=$ 3 or 5 , (B) same noise after applying spectral subtraction with the true $\sigma^{2}$, (C) same noise after $\log$ spectral subtraction with the true $\sigma^{2}$. Dots are 500 simulated points and purple lines are kernel density estimates. The numbers indicate the probability of exceeding the threshold $\lambda=3.84$ (5th percentile of $\chi_{1}^{2}$, orange line) in each case. See Supplementary Material B for calculation details.

monitoring project.

Over 2018-2019, nightly acoustic monitoring was conducted with passive recorders in Zealandia sanctuary, Wellington, New Zealand. We selected five nights of recordings from this data, obtained over various months using two devices (SM2, Wildlife Acoustics). Recorders were attached to trees at about 1-1.5 m above ground, with one located in a relatively exposed position on a hilltop, and the other in a sheltered valley. We extracted 3 audio clips of 0.1 seconds from each night and device, starting within one minute of 23:00, and manually verified that no distinct animal calls are heard in these clips. For comparison with a different hardware, we extracted 3 similar clips from one night from a different monitoring project, conducted in 2021 in Ponui island, New Zealand, using an AR4 recorder (Department of Conservation). All clips were resampled to $16000 \mathrm{~Hz}$.

The clips were subject to WPT using two different wavelets: discrete Meyer, which approximates ideal bandpassing, and order 8 Symlet, based on its estimation performance in Atto et al. (2010). Energy within each node $\left(\left|Y_{j, k, t}\right|^{2}\right.$ using the notation above) was averaged over the $0.1 \mathrm{~s}$ clip and shown as the spectrum estimate at node centre frequency $f_{j, k}$. For comparison, we also plotted the spectra obtained from the clips by periodogram, Daniell-smoothed with a 7-bin kernel and downsampled.

OLS regression models were then fitted to the log frequencies vs. log energies, as 
in (5). We used either all nodes between 150-7500 Hz ("full spectrum", excludes only edge bands that have filtering effects) or nodes between $150-6000 \mathrm{~Hz}$, to focus on the more likely wind range. A series of models were fitted, from linear to 6th degree polynomial, and evaluated by the small-sample corrected Akaike criterion: AICc $=-2 \log L+2 k n /(n-k-1)$.

\subsection{Case study: applying the proposed noise-robust detection}

We demonstrate the proposed wind-robust detection method on two bird surveys. The first is a survey of Australasian bittern (Botaurus poiciloptilus), conducted near Lake Ellesmere, New Zealand. The male bitterns emit 'boom' calls at low frequency (around $150 \mathrm{~Hz}$ ), meaning that their detection is particularly affected by wind. The survey was conducted for 2 hours using 7 recorders at $8 \mathrm{kHz}$ sampling rate. Playback was used to solicit calls, and for the purpose of method evaluation we count both playback and responses as true calls. The second survey is of little spotted kiwi (Apteryx owenii) in Zealandia wildlife sanctuary, New Zealand. Male kiwi calls are a sequence of around 20 repeated syllables in the $2-3 \mathrm{kHz}$ band. Eight recorders with 6 hours of sound from each were used. These surveys were previously used to evaluate sound detection methods in Juodakis et al. (2021b), and further details about this data are provided therein.

The recordings were analysed using the changepoint detector from Juodakis et al. (2021b). Briefly, a training process uses a small number of annotated files to characterize the wavelet nodes and duration of each species calls. The survey files are then analysed to detect periods of increased energy in these nodes. The detector can adapt to long-term changes in background level, but transient events such as wind are not removed and cause false positives (Juodakis et al., 2021b). The windadjusted analyses use the same detectors with the same parameter settings, but reduce noise level by log-spectral subtraction as described here. The analysis was repeated using either the OLS or QR noise estimate. The same window length is used for both detection and fitting of the noise spectrum models. For the quantile estimate $\hat{N}^{Q, \tau}$, we set $\tau=0.2$, and in the case of bittern the estimate was adjusted upwards by 0.4 (this factor, based on (6), is typically negligible and only used here because of the low sampling rate of the bittern recordings).

Evaluation is based on the precision of a spatial capture-recapture model (SCR), as proposed previously in Juodakis et al. (2021a). SCR is a general framework for inferring population density from imperfectly detected cues (Dawson and Efford, 2009). Its key component is a detection function $p(d)$, modelling the probability of 
detecting calls emitted at distance $d$ from the recorder. In the grid-based SCR, as used here, this probability is estimated from calls simultaneously detected by more than one recorder. Another option is to calibrate $p(d)$ from external data, in which case the SCR reduces to the distance sampling model (Borchers et al., 2015). The density of animal calls, assumed proportional to the density of animals, is estimated using this $p(d)$. As this density is the main target of ecological interest, we use its standard error (SE) to evaluate the detection methods.

After applying the detection algorithms, equal number of reported segments from each method were reviewed manually. The verified detections were used to fit an SCR model, and the density SE estimated by bootstrap (Stevenson et al., 2015) is reported, as well as the coefficient of variation $S E(\hat{D}) / \hat{D}$ to allow differences in

the density estimate $\hat{D}$. We refer the reader to Borchers et al. (2015); Stevenson et al. (2015) for a full introduction to acoustic SCR, and to Juodakis et al. (2021a) or Juodakis et al. (2021b) for details on formatting data for this type of model.

\subsection{Using the noise estimate to restore clean sound}

The proposed estimator of broadband noise level $\left(\hat{N}^{O L S}\right.$ or $\left.\hat{N}^{Q, \tau}\right)$ can also be combined with other sound analysis methods, not only detectors. We demonstrate how it can be used for restoring clean sound by wavelet shrinkage.

Wavelet shrinkage by soft-thresholding is a popular denoising method (Donoho, 1995). The soft-thresholding modifies the WPT coefficients $Y_{j, k, t}$ by translating them towards 0:

$$
\tilde{Y}_{j, k, t}=\operatorname{sign}\left(Y_{j, k, t}\right) \max \left(0,\left|Y_{j, k, t}\right|-\lambda \sigma_{j, k, t}\right)
$$

where $\sigma_{j, k, t}$ is some estimate of the noise SD in the node $j, k$, and $\lambda$ tunes the strength of the thresholding. This is based on the assumption that target signal energy will be concentrated in only a few coefficients after the wavelet transform, and so shrinking all coefficients will mostly reduce noise. The adjusted WPT is then inverted to reconstruct a denoised sound waveform (see e.g., (Wornell, 1993)), which is a simple process compared to inverting a spectrogram.

As a test, we create noisy files by mixing 2 min clips of windy background with bird sound examples. Background clips (5 files) were selected from Zealandia monitoring data. Bird sounds were 6 clips from the xeno-canto database and 6 clips of rich soundscapes from Zealandia monitoring. The xeno-canto examples were chosen to have a clear foreground and low background noise, because evaluating the denoising requires clean reference sounds. The Zealandia examples were taken 
from dawn or dusk choruses, to capture rich soundscapes that are difficult to denoise, although they have non-negligible background noise, which may impact the subsequent denoising metrics. The clips were mixed at $+12 \mathrm{~dB}, 0 \mathrm{~dB}$, or -12 SNR (the latter was only used with xeno-canto examples, as the soundscapes are too quiet to produce audible residual signal then), producing $300 \mathrm{~min}$ of noisy sound in total.

Each file was then analysed by constructing the WPT, and for each time window noise level estimates $\hat{N}^{O L S}, \hat{N}^{Q, \tau}$ were obtained by fitting a cubic polynomial to the WPT as described above. Since these values estimate the log-energies of noise, we can obtain adaptive estimates of $\sigma_{j, k, t}$ as $\sqrt{\exp \left(\hat{N}^{O L S}\right)}$ or $\sqrt{\exp \left(\hat{N}^{Q, \tau}\right)}$. We use these, with $\lambda=1$, in (10) to obtain OLS-denoised or QR-denoised coefficients. For comparison, we test a constant threshold with $\lambda=3$ and $\sigma_{j, k, t}=$ $\sum_{i=1}^{n}\left|Y_{j, k, i}-\operatorname{median}\left(Y_{j, k, i}\right)\right| /(0.6745 n)$; this is a robust estimate of the noise SD, commonly recommended for wavelet shrinkage, and leading to various optimal theoretical properties (Donoho, 1995). The resulting adjusted WPT was inverted to reconstruct the denoised sound file following standard wavelet methods (Donoho, 1995).

The success of denoising was evaluated by estimating the SNR improvement in $\mathrm{dB}$ :

$S N R_{\text {impr. }}=S N R_{\text {denoised }}-S N R_{\text {noisy }}=10 \log _{10} \sum_{t} X_{t}^{2} /\left(X_{t}-\hat{X}_{t}\right)^{2}-10 \log _{10} \sum_{t} X_{t}^{2} /\left(X_{t}-X_{t}^{N}\right)^{2}$,

where $X_{t}, X_{t}^{N}$ and $\hat{X}_{t}$ are the clean, noisy and denoised waveforms of a file. We also calculate the SI-SDR, which is a robust modification of SNR that is invariant to scale changes introduced during denoising (Roux et al., 2019).

\section{Results}

\subsection{Field recordings indicate non-linear background spectra}

The pilot dataset revealed the presence of a variety of background noise spectra (Figure 3A). Overall, the noise power was higher in lower frequencies, higher in the January and March nights when more wind gusts were audible, and more variable for the recorder in an exposed location, which is in line with the $1 / f$ model (note that some files have an additional peak corresponding to strong cicada noise, at around $3000 \mathrm{~Hz}$ ). Spectra taken close in time to each other show little variation, 
suggesting that the estimation is precise in stable conditions.

However, over longer periods, spectral shapes varied considerably, deviating from the predicted log-log line. Even within the same device and same minute, wind gusts caused some considerable changes in spectrum shape (see top lines in Figure 3A, "windy" recorder). Similar spectra were obtained with a different wavelet, or by a smoothed periodogram (Supplementary Figure S6), indicating that the shape variation is not caused by the chosen estimation method.

Linear models were also not supported by the fit statistics: when fitting the full-spectrum, average AICc for the linear model was 35.0, while the higher order polynomials had AICc between 17.0-23.6. Even if the frequency range is limited to $<6000 \mathrm{~Hz}$, the linear model is still insufficient (AICc 28.5, but 16.5-21.3 for higher order models). The optimal model degree by this criterion was 5 (for full spectrum) or 3 (for $<6000 \mathrm{~Hz}$ ). Some examples of 3rd degree and linear spectrum fits are shown in Figure 3B-C. Based on these results, we chose to use a 3rd degree model fitted to $<6000 \mathrm{~Hz}$ spectrum in the detector, as it seems to provide sufficient flexibility in the range where wind noise is the most prominent, without great sensitivity to interferences or large computational cost.
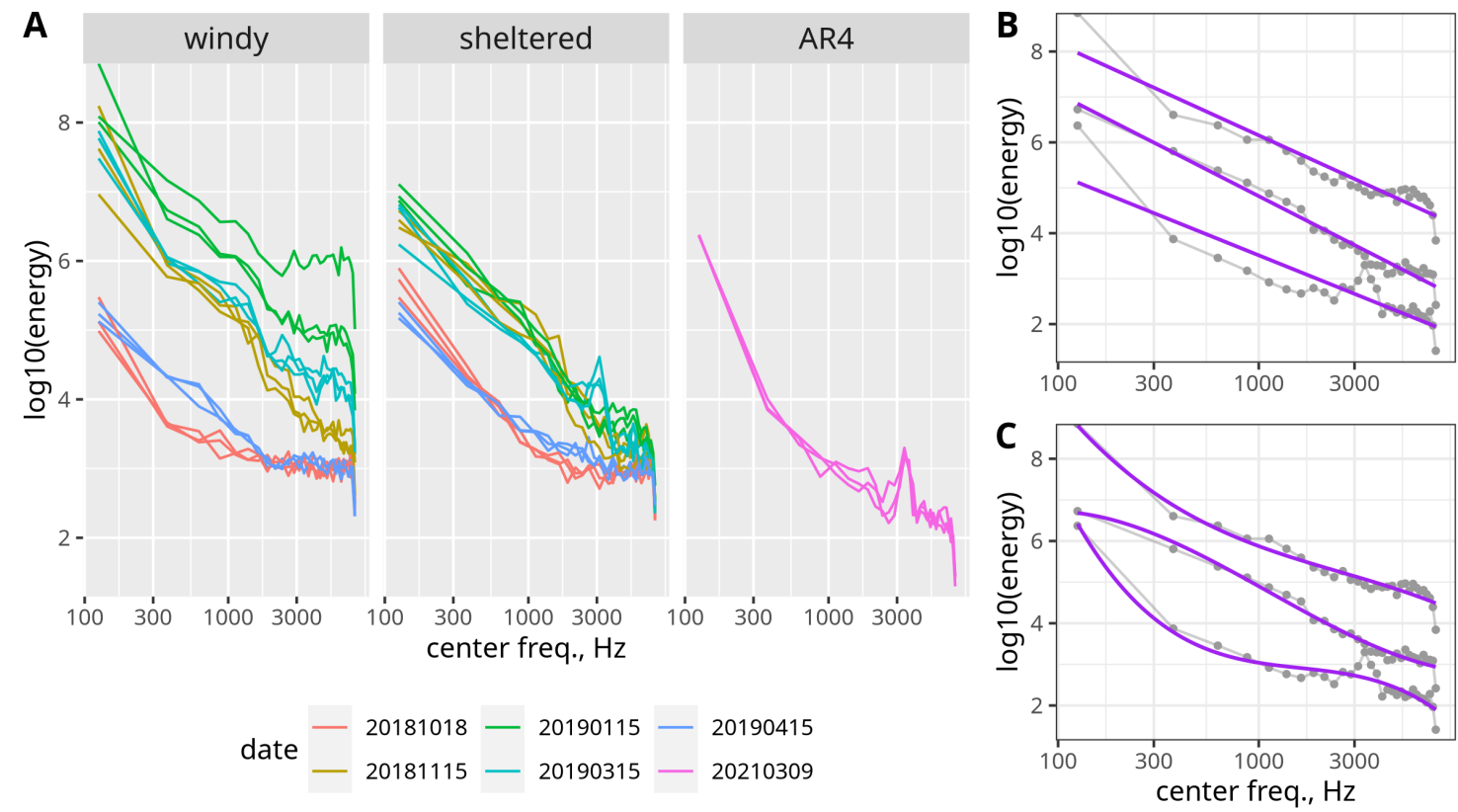

Figure 3: (A) Short-term spectra (energies in discrete Meyer wavelet packet nodes) of 0.1 $s$ clips from passive acoustic recordings. The log energy of each node is plotted against its centre frequency. Panels show clips from a recorder in a windy location, a sheltered location, and a different model recorder (AR4). (B) A subset of three example spectra (grey connected points) overlaid with linear fits. (C) Same example clips with cubic fits. 


\subsection{Evaluating robust detection on surveys}

In all tested settings, we observed that wind adjustment greatly reduced the number of false positives. In the bittern survey, 859 detections were obtained using the OLS-adjusted method. Without the adjustment, 1505 segments were reported, of which 57\% were reviewed to equalize the effort across both methods. Most of the additional false positives in this set were indeed wind or other broadband noises such as plane overflights, which were also removed by the proposed adjustment. After a review stage to remove false positives, fitting SCR models to the two sets of verified detections confirms that the adjustment greatly improves survey efficiency, with about two-fold lower coefficient of variation for the estimated density (Table 1).

An even greater contrast is seen in the kiwi survey. With the same thresholds, the adjusted detection resulted in so few false positives that it required extreme downsampling of the unadjusted data (SCR models could not be reliably fitted). Therefore the results shown here use a two times smaller threshold for the adjusted detector. This produced 323 detections, mostly true positives, with the rest caused by sounds of other species in the target bands, such as the kaka parrot (Nestor meridionalis). In comparison, the unadjusted detector produced 1315 detections (25\% reviewed), and 4 times less precise density estimates after human review (CV $52.5 \%$ vs $12.2 \%$, Table 1 ).

Table 1: Detection results from two bird surveys, obtained using a wavelet changepoint detector with or without a wind noise adjustment. The adjustment uses the OLS spectrum fit presented in this paper. The main evaluation metrics are highlighted: standard error (SE) or the coefficient of variation (CV) of the survey density estimate. Also shown are the estimates of the density itself and of the detection radius parameter $\sigma$.

\begin{tabular}{l|rr|rr}
\hline & \multicolumn{2}{|c|}{ Bittern } & \multicolumn{2}{c}{ Kiwi } \\
& no adj. & OLS adj. & no adj. & OLS adj. \\
\hline Total detections & 1505 & 859 & 1315 & 323 \\
$\sigma(\mathrm{m})$ & 329 & 263 & 290 & 232 \\
Density (calls/ha) & 0.36 & 0.52 & 1.08 & 1.03 \\
Density SE & 0.35 & 0.27 & 0.55 & 0.13 \\
Density CV (\%) & 99.5 & $\mathbf{5 2 . 5}$ & 51.1 & $\mathbf{1 2 . 2}$ \\
\hline
\end{tabular}

When estimating wind noise by quantile regression instead of OLS, slightly more false positives were produced, with 1025 total detections for bittern and 360 for kiwi. The detected segments mostly matched those reported by OLS, so we do not analyse these further here. 


\subsection{Incorporating the wind estimator into denoising}

The proposed wind noise level estimators are useful for denoising as well. Wavelet shrinkage with wind-adaptive thresholds, either estimated by OLS or by QR, considerably improved the SNR (Figure 4). In contrast, the same denoising method with a constant threshold led to very little improvement: while it decreased the overall background noise, most of the noise energy in these examples came from wind gusts, which this method could not remove. Note that in some cases SNR even apparently decreased: because some white noise was present in the "clean" recordings as well, removing that decreased the measured match between the reference and denoised files, and was thus counted as a loss of signal by this metric. (This effect also contributed to the lower denoising performance seen when using the soundscape references, which had more residual noise than the xeno-canto clips.)

Similar results are seen when using the SI-SDR metric (Supplementary Figure S7). As this metric is invariant to the initial mixing SNR, it produces more uniform measures over the tested files, removing the spurious improvement peak seen at 0 $\mathrm{dB}$ with the xeno-canto examples. The difference between OLS and QR estimation methods was very small in the metrics used here, although in favour of $Q R$ in every case.

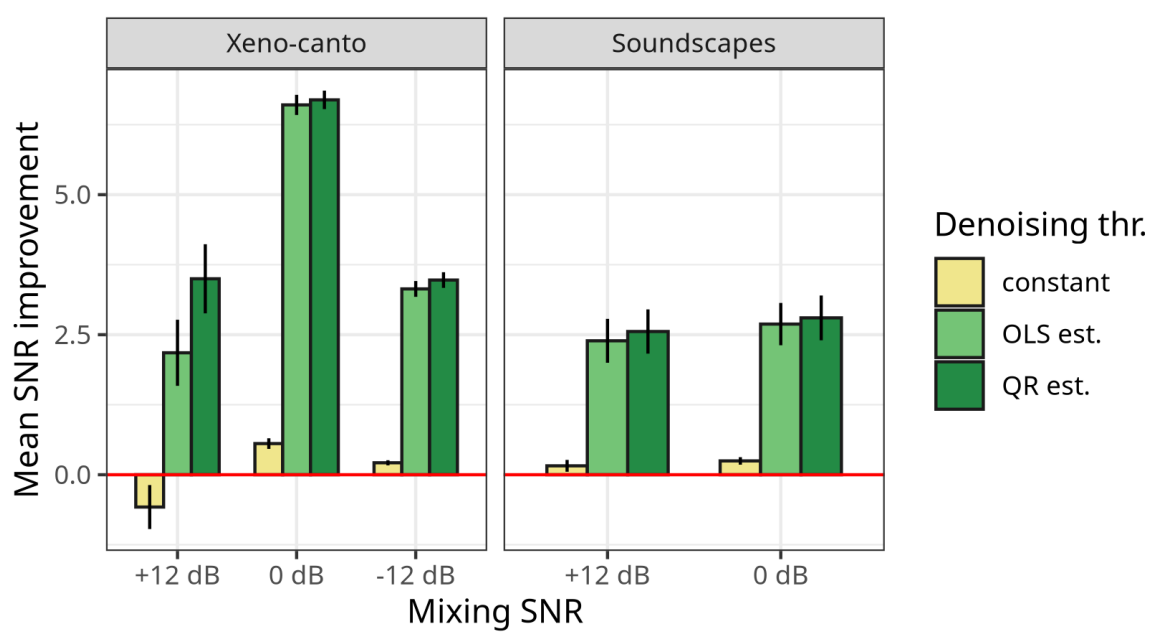

Figure 4: Average SNR improvement obtained with different wavelet denoising thresholds: constant, or based on the time-varying OLS or QR spectrum models, as presented here. Either high-SNR recordings of individual birds (xeno-canto) or dense soundscapes from passive monitoring data were mixed with wind noise at different SNRs, and denoised by wavelet shrinkage. Error bars show $\pm 1 \mathrm{SE}$.

To gain some insight into the working of each method, we show spectrograms of the denoised outputs from one example clip in Figure 5. Denoising with a constant 
threshold successfully removed the more stable parts of the background noise, as indicated by uniform grey areas in Figure 5A; however, it had very little effect on the wind gust seen around $10 \mathrm{~s}$ from the start of the clip. Both of the time-varying estimators successfully modelled this gust, which led to its removal. The main differences between the estimation by OLS and QR is seen during the time periods when loud calls are present: as predicted, these calls affect the OLS fit more, and cause over-adjustment (grey gaps) or under-adjustment (green residual nosie) in the $0-2 \mathrm{kHz}$ frequency range (Fig. 5B). The QR estimate was robust to these effects (Fig. 5C).

\section{Discussion}

\subsection{Summary and alternative design considerations}

In this study, we proposed a new noise estimator based on fitting a polynomial model to wavelet packet node energies. This estimator was combined with log spectral subtraction to stabilize the noise level. In our case study this adjustment greatly reduced the number of false positive detections and led to more efficient acoustic surveys. Additionally, we showed that the estimator can be incorporated in a wavelet denoising method to restore sound polluted by broadband noise.

Although our initial motivation was wind noise, which in theory is associated with a specific (log-)linear spectrum shape, the pilot experiment indicated that a more flexible model was needed. The resulting polynomial estimator now also captures more general broadband noises besides wind. This is useful for our surveys, but in other cases the target signal may be broadband, such as insect stridulations (Field and Rind, 1992). Our method is thus limited to signals with characteristic frequency bands. However, choosing other filterbanks instead of the wavelet packet, such as the Mel, gammatone or Greenwood (Zeppelzauer et al., 2015), may concentrate different sounds better, thus allowing analysis of a wide variety of tasks.

We proposed to fit the spectrum using quantile regression to account for asymmetric contamination when other signals are present. Nonetheless, the standard OLS fitting appeared surprisingly robust, although choosing $Q R$ is safer when the soundscape is particularly rich, or precise noise estimation around calls is important. This may be a useful precursor step for automatic analysis of dawn choruses, in which the high density of calls presents a challenge for current detection soft- 

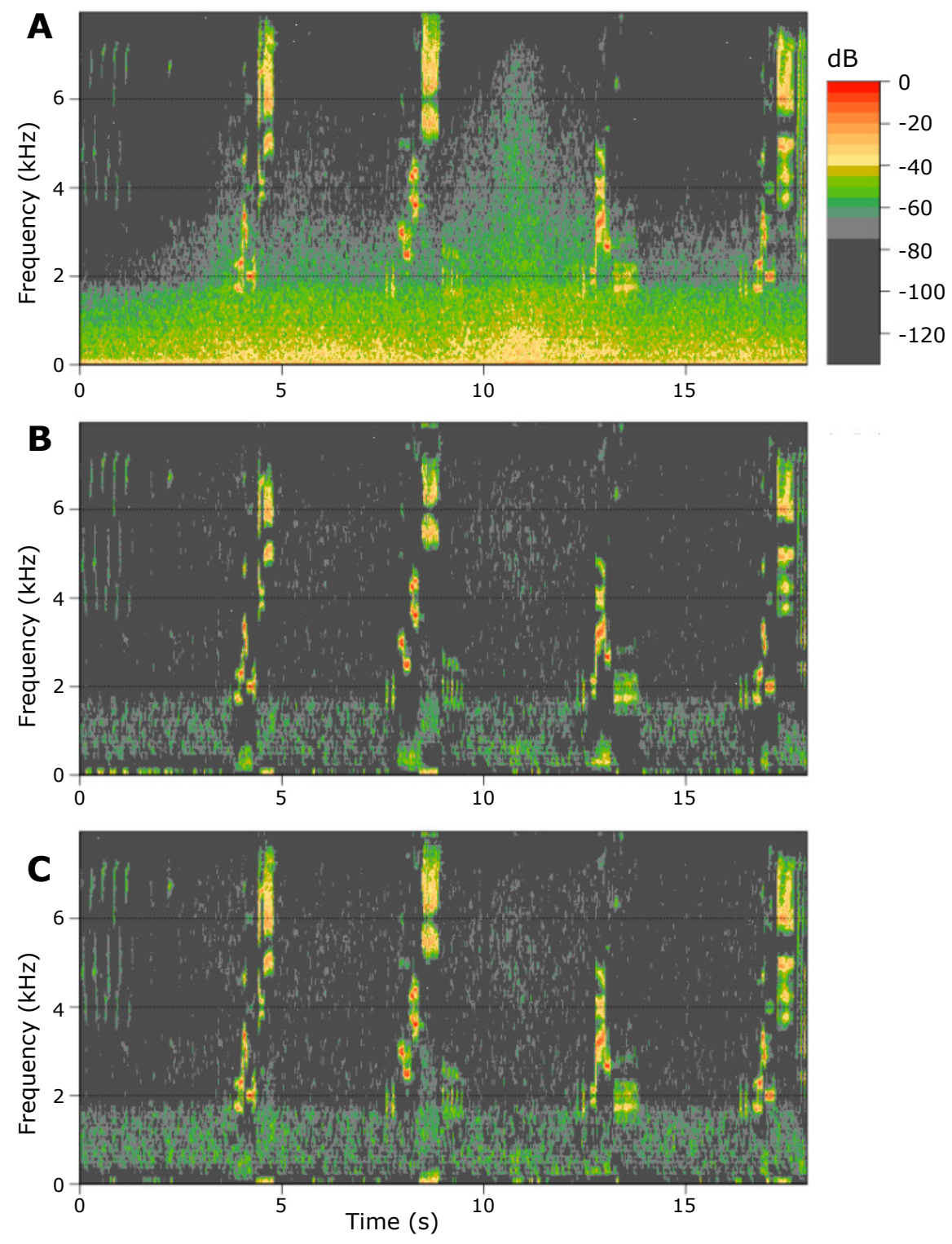

Figure 5: Recording of a wood thrush Hylocichla mustelina, xeno-canto ID XC561864, mixed with wind noise at $+12 \mathrm{~dB}$, and denoised by wavelet shrinkage with different thresholds: (A) constant threshold, (B) adaptive threshold estimated by OLS, (C) adaptive threshold estimated by QR.

ware (Brooker et al., 2020).

\subsection{Differences from other noise estimators}

The surveys analysed here highlight some of the issues with applying other noise estimation methods in bioacoustics. Since our spectrum model uses short time windows (on the order of $0.1 \mathrm{~s}$ ) and is not smoothed over time, it can adapt to fast 
transients, while methods such as PCEN (Lostanlen et al., 2019) or MMSE-STSA (Brown et al., 2018) critically rely on the noise changing more slowly than the signal, so would be unusable with the c. 30-second-long kiwi call. Methods designed to remove low-frequency noises, such as presented in Nelke et al. (2014), cannot be applied to the $150 \mathrm{~Hz}$ bittern sounds, and in general require knowledge of the other signals expected in the environment. In contrast, the proposed noise estimator needs very little tuning to be applied to different species: the main parameter is the frequency range of the target, which is retrieved from the detection stage. Furthermore, it can often be used even without specifying the signal bands at all, as shown in the denoising examples.

Thus, the proposed framework is designed for low training data situations that are common in wildlife research, where recording collection and expert annotation is expensive. In our survey analysis workflow, only the wavelet energy detector needs training, which has a simple structure and so can be trained with less than an hour of data (Priyadarshani et al., 2020; Juodakis et al., 2021b). Neural networks could be used to create noise-robust detectors that outperform our results if given sufficient data, but this likely means at least thousands of clips, as in e.g., Vickers et al. (2021). Options to reduce this requirement, such as by transfer learning or using weak labels (Serizel et al., 2018), are actively researched. Even with that, our method will still remain useful, as it provides a fast and robust initial screening step at very little cost in terms of missed calls, and its output can be verified by a more sophisticated procedure if available.

Additionally, we have used a wavelet transform throughout all stages of the sound analysis. This transform can be easily inverted, as we have done here to recreate the denoised audio files, whereas most other methods produce spectrograms, inverting which would be more complicated (Zhu et al., 2007).

\subsection{Evaluating the improvements in practice}

The metrics chosen to evaluate the proposed methods may not represent all practical needs. For the detection stage, we conducted a grid-based survey in the SCR framework and measured its efficiency as proposed in Juodakis et al. (2021a). Alternative measures, such as the F-score, are common in the acoustic detection community (see e.g., Priyadarshani et al. (2020)). In contrast to these, our SCR metric directly measures the precision of the estimate of interest, and thus the power to conduct ecologically relevant comparisons. To the best of our knowledge, this study is the first to explicitly demonstrate that survey efficiency is gained by using 
noise-robust sound analysis methods. The metric is also quite general, as various bioacoustic survey designs can be expressed as special cases of SCR (Borchers et al., 2015). Ultimately, in the present case, the robust detector showed much lower false alarm rate with almost no loss in true detections, so it should be identified as an improvement by most metrics.

It is yet more complicated to evaluate the benefits of denoising in bioacoustics. If the cleaned sound is used for human listening, presence of perceptual artefacts such as musical noise may be more important than SNR (Vaseghi, 2009). Metrics such as PESQ have been designed to capture the subjective quality of sound (Rix et al., 2001), but they rely on speech-specific properties and do not directly apply to other species. In the context of ecological monitoring, the primary application of denoising currently is to improve the classification of calls by neural networks, as in e.g., Vickers et al. (2021). Deep learning is also suggested for more holistic ecoacoustic assessments, outside traditional surveys, and removing noise is also of interest there (Fairbrass et al., 2018). Because of the black-box nature of these methods and variety in the network and training setups, it is not clear whether SNR, PESQ or other metrics would actually be predictive of their performance. Standardizing the protocols of training and applying neural networks in bioacoustics would allow one to investigate this relationship, and to further develop denoising methods that are beneficial in ecology practice.

\section{Bibliography}

Achard, S. and Coeurjolly, J.-F. (2010) Discrete variations of the fractional Brownian motion in the presence of outliers and an additive noise. Statistics Surveys, 4, 117-147.

Astaras, C., Linder, J. M., Wrege, P., Orume, R. D. and Macdonald, D. W. (2017) Passive acoustic monitoring as a law enforcement tool for Afrotropical rainforests. Frontiers in Ecology and the Environment, 15, 233-234.

Atto, A. and Pastor, D. (2010) Central limit theorems for wavelet packet decompositions of stationary random processes. IEEE Transactions on Signal Processing, 58, 896-901.

Atto, A. M., Pastor, D. and Mercier, G. (2010) Wavelet packets of fractional Brownian motion: Asymptotic analysis and spectrum estimation. IEEE Transactions on Information Theory, 56, 4741-4753.

Borchers, D. L., Stevenson, B. C., Kidney, D., Thomas, L. and Marques, T. A. (2015) A unifying model for capture-recapture and distance sampling surveys of wildlife populations. Journal of the American Statistical Association, 110, 195-204.

Brooker, S. A., Stephens, P. A., Whittingham, M. J. and Willis, S. G. (2020) Automated 
detection and classification of birdsong: An ensemble approach. Ecological Indicators, 117, 106609.

Brown, A., Garg, S. and Montgomery, J. (2018) Automatic and efficient denoising of bioacoustics recordings using MMSE STSA. IEEE Access, 6, 5010-5022.

Chen, Y. (2010) Improved energy detector for random signals in Gaussian noise. IEEE Transactions on Wireless Communications, 9, 558-563.

Dawson, D. K. and Efford, M. G. (2009) Bird population density estimated from acoustic signals. Journal of Applied Ecology, 46, 1201-1209.

Digby, A., Towsey, M., Bell, B. D. and Teal, P. D. (2013) A practical comparison of manual and autonomous methods for acoustic monitoring. Methods in Ecology and Evolution, 4, 675-683.

Donoho, D. (1995) De-noising by soft-thresholding. IEEE Transactions on Information Theory, 41, 613-627.

Fairbrass, A. J., Firman, M., Williams, C., Brostow, G. J., Titheridge, H. and Jones, K. E. (2018) CityNet-deep learning tools for urban ecoacoustic assessment. Methods in Ecology and Evolution, 10, 186-197.

Field, L. and Rind, F. (1992) Stridulatory behaviour in a New Zealand weta, Hemideina crassidens. Journal of Zoology, 228, 371-394.

Juodakis, J., Castro, I. and Marsland, S. (2021a) Precision as a metric for acoustic survey design using occupancy or spatial capture-recapture. Environmental and Ecological Statistics, 28, 587-608.

Juodakis, J., Marsland, S. and Priyadarshani, N. (2021b) A changepoint prefilter for sound event detection in long-term bioacoustic recordings. The Journal of the Acoustical Society of America, 150, 2469-2478.

Kahl, S., Stöter, F.-R., Goëau, H., Glotin, H., Planque, R., Vellinga, W.-P. and Joly, A. (2019) Overview of BirdCLEF 2019: Large-Scale Bird Recognition in Soundscapes. In CLEF 2019 Working Notes, vol. 2380 of CEUR Workshop Proceedings, 1-9. Cappellato, L. and Ferro, N. and Losada, D. E. and Müller, H., Lugano, Switzerland: CEUR.

Keshavarzi, M., Goehring, T., Zakis, J., Turner, R. E. and Moore, B. C. J. (2018) Use of a deep recurrent neural network to reduce wind noise: Effects on judged speech intelligibility and sound quality. Trends in Hearing, 22.

Koenker, R. and Bassett, G. (1978) Regression quantiles. Econometrica, 46, 33.

Lasseck, M. (2013) Bird song classification in field recordings: Winning solution for NIPS4B 2013 competition. In Proceedings of 'Neural Information Processing Scaled for Bioacoustics: From Neurons to Big Data - NIP4B', 176-181.

Lostanlen, V., Salamon, J., Cartwright, M., McFee, B., Farnsworth, A., Kelling, S. and Bello, J. P. (2019) Per-channel energy normalization: Why and how. IEEE Signal Processing Letters, 26, 39-43. 
Mcloughlin, M. P., Stewart, R. and McElligott, A. G. (2019) Automated bioacoustics: methods in ecology and conservation and their potential for animal welfare monitoring. Journal of The Royal Society Interface, 16, 20190225.

Metcalf, O. C., Lees, A. C., Barlow, J., Marsden, S. J. and Devenish, C. (2020) hardRain: An $\mathrm{R}$ package for quick, automated rainfall detection in ecoacoustic datasets using a threshold-based approach. Ecological Indicators, 109, 105793.

Moulines, E., Roueff, F. and Taqqu, M. S. (2007) On the spectral density of the wavelet coefficients of long-memory time series with application to the log-regression estimation of the memory parameter. Journal of Time Series Analysis, 28, 155-187.

Nelke, C. (2016) Wind noise reduction: signal processing concepts. Ph.D. thesis, RWTH Aachen University, Germany.

Nelke, C. M., Chatlani, N., Beaugeant, C. and Vary, P. (2014) Single microphone wind noise PSD estimation using signal centroids. In 2014 IEEE International Conference on Acoustics, Speech and Signal Processing (ICASSP). IEEE.

Nelke, C. M. and Vary, P. (2015) Wind noise short term power spectrum estimation using pitch adaptive inverse binary masks. In 2015 IEEE International Conference on Acoustics, Speech and Signal Processing (ICASSP). IEEE.

Page, E. S. (1954) Continuous inspection schemes. Biometrika, 41, 100.

Prince, P., Hill, A., Covarrubias, E. P., Doncaster, P., Snaddon, J. and Rogers, A. (2019) Deploying acoustic detection algorithms on low-cost, open-source acoustic sensors for environmental monitoring. Sensors, 19, 553.

Priyadarshani, N., Marsland, S. and Castro, I. (2018) Automated birdsong recognition in complex acoustic environments: a review. Journal of Avian Biology, 49, jav-01447.

Priyadarshani, N., Marsland, S., Juodakis, J., Castro, I. and Listanti, V. (2020) Wavelet filters for automated recognition of birdsong in long-time field recordings. Methods in Ecology and Evolution, 11, 403-417.

Rhinehart, T. A., Chronister, L. M., Devlin, T. and Kitzes, J. (2020) Acoustic localization of terrestrial wildlife: Current practices and future opportunities. Ecology and Evolution, 10, 6794-6818.

Rix, A., Beerends, J., Hollier, M. and Hekstra, A. (2001) Perceptual evaluation of speech quality (PESQ)-a new method for speech quality assessment of telephone networks and codecs. In 2001 IEEE International Conference on Acoustics, Speech, and Signal Processing. Proceedings (Cat. No.01CH37221). IEEE.

Roux, J. L., Wisdom, S., Erdogan, H. and Hershey, J. R. (2019) SDR - half-baked or well done? In ICASSP 2019 - 2019 IEEE International Conference on Acoustics, Speech and Signal Processing (ICASSP). IEEE.

Serizel, R., Turpault, N., Eghbal-Zadeh, H. and Shah, A. P. (2018) Large-scale weakly labeled semi-supervised sound event detection in domestic environments. In Proceed- 
ings of the Detection and Classification of Acoustic Scenes and Events 2018 Workshop (DCASE2018), 19-23.

Serroukh, A., Walden, A. T. and Percival, D. B. (2000) Statistical properties and uses of the wavelet variance estimator for the scale analysis of time series. Journal of the American Statistical Association, 95, 184-196.

Shonfield, J. and Bayne, E. (2017) Autonomous recording units in avian ecological research: current use and future applications. Avian Conservation and Ecology, 12.

Stevenson, B. C., Borchers, D. L., Altwegg, R., Swift, R. J., Gillespie, D. M. and Measey, G. J. (2015) A general framework for animal density estimation from acoustic detections across a fixed microphone array. Methods in Ecology and Evolution, 6, 38-48.

Sugai, L. S. M., Silva, T. S. F., Ribeiro, J. W. and Llusia, D. (2018) Terrestrial passive acoustic monitoring: Review and perspectives. BioScience, 69, 15-25.

Vaseghi, S. V. (2009) Advanced Digital Signal Processing and Noise Reduction. Wiley.

Veitch, D. and Abry, P. (1999) A wavelet-based joint estimator of the parameters of longrange dependence. IEEE Transactions on Information Theory, 45, 878-897.

Vickers, W., Milner, B., Risch, D. and Lee, R. (2021) Robust North Atlantic right whale detection using deep learning models for denoising. The Journal of the Acoustical Society of America, 149, 3797-3812.

Walker, K. T. and Hedlin, M. A. (2009) A review of wind-noise reduction methodologies. In Infrasound Monitoring for Atmospheric Studies, 141-182. Springer Netherlands.

Wornell, G. W. (1993) Wavelet-based representations for the $1 / \mathrm{f}$ family of fractal processes. In Proceedings of the IEEE, vol. 81, 1428-1450. IEEE.

Zeppelzauer, M., Hensman, S. and Stoeger, A. S. (2015) Towards an automated acoustic detection system for free-ranging elephants. Bioacoustics, 24, 13-29.

Zhang, X. and Li, Y. (2015) Adaptive energy detection for bird sound detection in complex environments. Neurocomputing, 155, 108-116.

Zhu, X., Beauregard, G. T. and Wyse, L. L. (2007) Real-time signal estimation from modified short-time Fourier transform magnitude spectra. IEEE Transactions on Audio, Speech and Language Processing, 15, 1645-1653.

Znidersic, E., Towsey, M. W., Hand, C. and Watson, D. M. (2021) Eastern black rail detection using semi-automated analysis of long-duration acoustic recordings. Avian Conservation and Ecology, 16. 


\section{Supplementary material}

\section{A Estimation of quantiles with contamination}

Let $N$ be a random variable following a contaminated mixture distribution, i.e., its CDF is $F_{N}=(1-\epsilon) F_{X}+\epsilon F_{Z}$, with a contaminating variable $Z$ that is concentrated at larger values than $X$. Specifically, denoting the median of $X$ as $\mu_{X}$, define this requirement as:

$$
F_{Z}(x) \ll F_{X}(x) \text { for all } x \leq \mu_{X} .
$$

Then its $\tau$-quantile for $\tau /(1-\epsilon)<0.5$ is:

$$
\begin{aligned}
F_{N}^{-1}(\tau) & =\inf \left(x: F_{N}(x) \geq \tau\right) \\
& =\inf \left(x:(1-\epsilon) F_{X}(x)+\epsilon F_{Z}(x) \geq \tau\right) \\
& \approx \inf \left(x:(1-\epsilon) F_{X}(x)+0 \geq \tau\right. \\
& =\inf \left(x: F_{X}(x) \geq \tau /(1-\epsilon)\right) \\
& =F_{X}^{-1}(\tau /(1-\epsilon))
\end{aligned}
$$

The $N, X$ and $Z$ variables correspond to $\left|N_{j, k, t}\right|^{2}, c(t) f_{j, k}^{-\alpha} \chi_{1}^{2}$ and $Z_{j, k, t}$ from (7). Thus, the quantile regression estimate $\hat{N}^{Q, \tau}$, which converges to $F_{N}^{-1}(\tau)$ under standard regression conditions, also converges to the $\tau /(1-\epsilon)$ quantile of the "clean" distribution $c(t) f_{j, k}^{-\alpha} \chi_{1}^{2}$.

Naturally, neither $\epsilon$ nor the exact range of $\tau$ over which the requirement (S11) holds are known in advance. However, choosing a small quantile such as $\tau=0.2$ should work for most situations in practice: even under $50 \%$ contamination, this corresponds to $\tau /(1-\epsilon)=0.4<\mu_{X}$, and we can reasonably expect that most signals will significantly exceed the median of the background.

\section{B Tail probabilities of spectral subtraction estimates}

Let $X_{t}^{2}$ be a random process with $X_{t}^{2} \sim c(t) \chi_{1}^{2}$, where the noise strength $c(t)$ varies with time. Assume the expected value of the process at each time $t$, i.e., $\mathbb{E} X_{t}^{2}=c(t)$ is known exactly. We wish to find the tail probabilities of $\tilde{X}_{t}^{2}$, obtained by standard (power) spectral subtraction (9):

$$
\tilde{X}_{t}^{2}=\max \left(0, X_{t}^{2}-c(t)\right)
$$


This is by definition a left-censored variable, with pdf

$$
f_{\tilde{X}_{t}^{2}}(x)= \begin{cases}f_{X_{t}^{2}-c(t)}(x)=f_{X_{t}^{2}}(x+c(t)) & \text { if } x>0 \\ F_{X_{t}^{2}-c(t)}(0) & \text { if } x=0 .\end{cases}
$$

The tail probability for any $\lambda>0$ is

$$
\begin{aligned}
P\left(\tilde{X}_{t}^{2}>\lambda\right) & =\int_{\lambda}^{\infty} f_{X_{t}^{2}}(u+c(t)) d u \\
& =\int_{\lambda+c(t)}^{\infty} f_{X_{t}^{2}}(x) d x \\
& =1-F_{X_{t}^{2}}(\lambda+c(t)) \\
& =1-F_{\chi_{1}^{2}}\left(\frac{\lambda+c(t)}{c(t)}\right) .
\end{aligned}
$$

Note that in the denoising context, this "denoised" distribution still depends on the noise strength $c(t)$.

Under log spectral subtraction, we immediately have that $X_{t}^{2} / c(t) \sim c(t) / c(t) \chi_{1}^{2}=$ $\chi_{1}^{2}$, and thus the tail probabilities for any $\lambda>1$ are $1-F_{\chi_{1}^{2}}(\lambda)$. The rectification $\max (1, \cdot)$ can be omitted if the distribution properties for $\lambda<1$ are also relevant. 


\section{Supplementary figures}
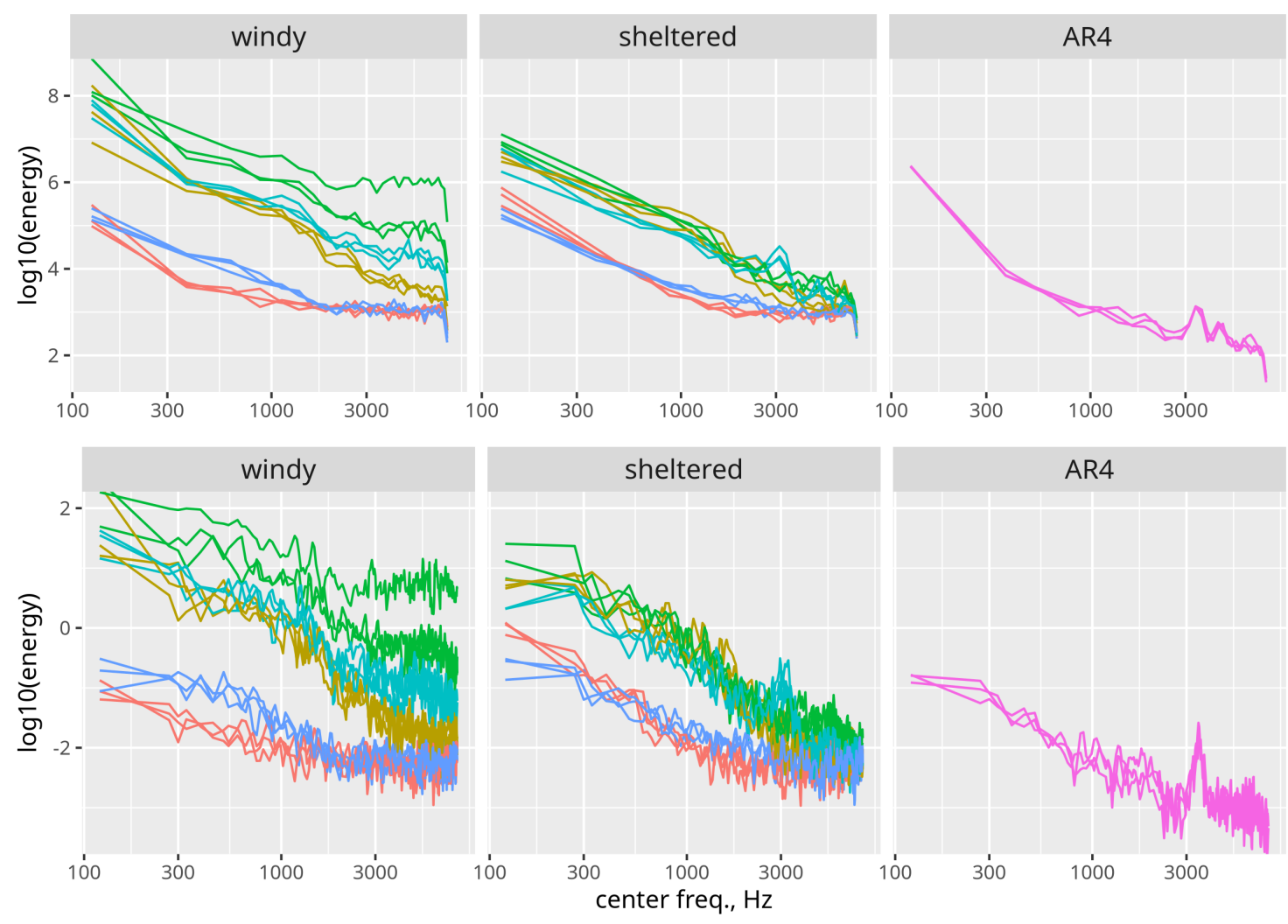

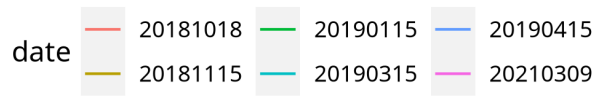

Figure S6: (Top row) Short-term spectra obtained using a Symlet(8) wavelet packet on $0.1 \mathrm{~s}$ clips from passive acoustic recordings. The log energy of each node is plotted against its centre frequency. Panels show clips from a recorder in a windy location, a sheltered location, and a different model of recorder (AR4). (Bottom row) Short-term spectra of the same clips estimated by periodogram, with Daniell smoothing over 7 bins. Note the considerably higher variance of this estimator, compared to wavelet packet spectra. 


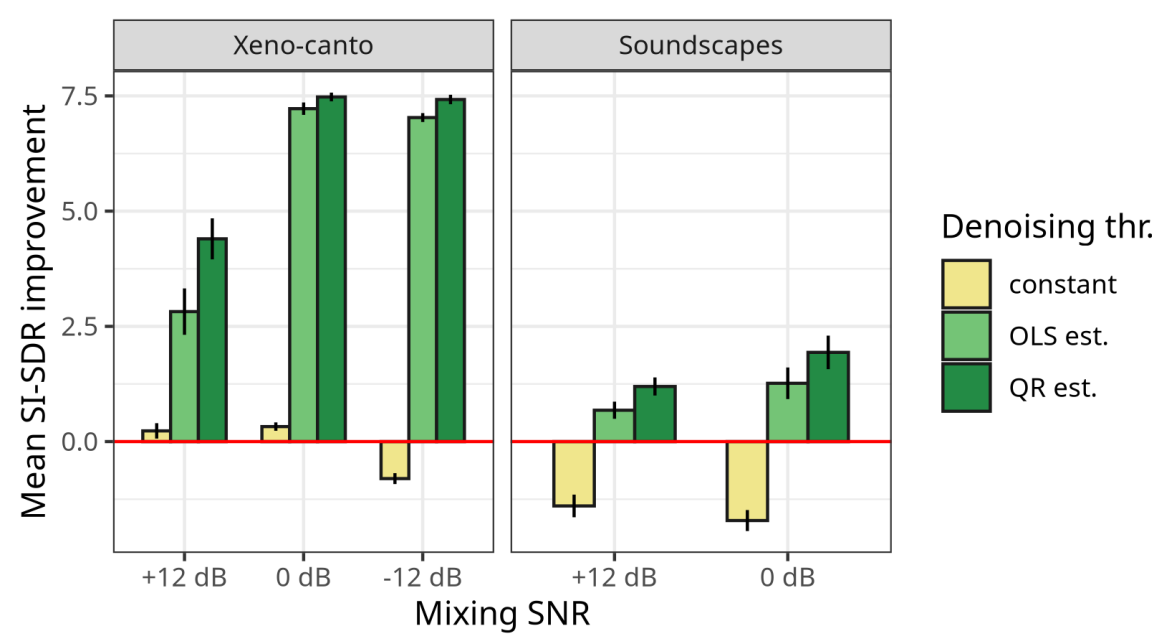

Figure S7: Average improvement in scale-invariant SDR obtained with different wavelet denoising thresholds: constant, or based on the time-varying OLS or QR spectrum models, as presented here. Either high-SNR recordings of individual birds (xeno-canto) or dense soundscapes from passive monitoring data were mixed with wind noise at different SNRs, and denoised by wavelet shrinkage. Error bars show $\pm 1 \mathrm{SE}$. 


\section{Conclusions}

\section{Summary of the results}

We showed in this thesis that a statistically principled approach can improve bioacoustic studies in various ways.

The objective of Study I (Chapter 2) was to establish a framework for evaluating various methodological improvements in bioacoustics. Explicitly defining the design goals in terms of estimate precision revealed various problems with different metrics, and led us to choose SCR as the most appropriate framework. We also established the baseline performance of available tools, and used that to measure the improvements made throughout this thesis. While our primary motivation was evaluating new automated analysis methods, the results are much broader and applicable to various other changes in experiment design and data processing.

Secondly, we set out to design an improved sound event detection method. We identified that epidemic changepoint detection provides a framework that could include this task, with some modifications: Study II (Chapter 3) introduced a way to incorporate background dynamics, and Study III (Chapter 4) combined it with a wavelet decomposition to produce a frequency-specific sound detector. Choosing the changepoint framework allowed us to use existing theory and algorithms, as well as insights from stochastic gradient descent, to design and analyse our methods. The resulting detector is fast, more accurate than detectors manually crafted for particular species, and can be analysed in a theoretical framework that also suggests possible limitations and improvements.

The final objective was to mitigate the effects of transient broadband noise, which was a major source of interference in the previous studies. We achieved that in Study IV (Chapter 5), by using short-term spectrum regression models and a spectral-subtraction-like adjustment. The choice of this approach was suggested by the changepoint model used in detection, which is designed for constant-level sounds, and by the overall inference goals set by SCR. This denoising considerably improved the performance of sound detection, especially in windy conditions, and opened possibilities for further analyses based on the reconstructed clean sound.

The processing tools developed in this thesis have been incorporated into the 
AviaNZ software and are already being used to tackle ecological questions. The final pipeline allows efficient, almost fully automated analysis of natural soundscapes, especially for more distinctive species: in our experience with kiwi, several hours of data can be processed with only a few minutes of manual effort. Achieving this required a combination of statistical insights, but also an in-depth understanding of practical needs to engage the broader conservation community. We hope that this thesis encourages other researchers to adopt this approach, and to continue developing statistically principled sound processing.

\section{Future directions}

During this work, several issues have emerged that will be relevant to bioacoustic analysis in the near future, in particular:

- adapting the detection methods to complex sounds that cover a wide frequency range;

- understanding how the segmentation and other pre-processing interacts with neural network classification;

- creating similar tools for inference frameworks that do not require call timestamps.

In this section, we will summarise some of our insights and pilot results that may contribute to investigating these topics.

\subsection{Multivariate wavelet pre-filter}

In studies II and III, we have described and analysed changepoint models for univariate series, specifically representing the energies of a single frequency band in the case of sound. Many calls are more complex than that, occupying several noncontiguous frequency bands at the same time (harmonics), or jumping between bands during the call (melodic motion). Such cases correspond to the multivariate changepoint detection problem, in which the observations at each time $t$ are a vector of energies over $p$ target bands, $\mathbf{x}_{t}=\left[x_{t, 1}, \ldots, x_{t, p}\right]$. In effect, the current procedure determines the presence of segment $[t, T]$ in each series $j$ by testing a statistic $C_{t}^{j}$, based on comparing the $x_{1: t, j}$ to $x_{t+1: T, j}$ (such as the CUSUM for mean changes). When $p>1$, we process each series independently this way, and merge 
any overlapping segments to simplify the review. Instead, methods specifically designed for multivariate detection could be applied; these combine the raw statistics instead of the segments, reasoning that this should improve the power, as shown in Zhang et al. (2010).

Specifically, several different ways of combining the band-level statistics could be useful for different purposes. Zhang et al. (2010) define $Z_{t}^{\mathrm{SUM}}=\sum_{j=1}^{p}\left(C_{t}^{j}\right)^{2}$, which is optimised for a simultaneous change across many bands (i.e. harmonics). Melodic sounds correspond to sparse changes, as at each time only one band is occupied, and therefore statistics based on extremum values would be more appropriate, such as $Z_{t}^{\mathrm{MAX}}=\max _{j \in 1, \ldots, p}\left(C_{t}^{j}\right)^{2}$ (Jirak, 2015). In our workflow, the $p$ target bands are still a small subset of the possible frequencies, selected during a training stage for each analysed species. Modern changepoint literature has also tackled problems where $p$ is large, and the relevant dimensions need to be automatically selected for each event: for example, in Cho (2016), the change statistics at each time point are treated as a series $\mathbf{C}_{t}=\left[C_{t}^{1}, \ldots, C_{t}^{p}\right]$, and subjected to another round of CUSUM to detect the changing bands. Such methods could be used to create a non-specific sound event detector that bypasses the training of bands entirely.

\subsection{What helps neural networks?}

An active direction of current research is investigating how various pre-processing or denoising options help (or impede) further sound analysis by neural networks (see, for example, Knight et al. (2019); Merchan et al. (2020); Vickers et al. (2021)). While such comparisons are entirely feasible within our SCR framework from Study I, they are laborious, and would require empirically testing the effects across the many available neural network types, species, training and testing conditions. This would be easier if proxy metrics were available that determine how well a sound clip captures the target's features that are important for perceptual or CNN-based distinction. Such metrics would also help develop denoising methods - we encountered this issue in Study IV, where we used basic SNR because of lack of better tools.

To solve the same issue in speech processing, metrics such as PESQ (Rix et al., 2001) have been developed, which use a complex combination of features to approximate the perceived sound quality. However, they do not seem to generalise outside of speech: Figure 1 shows these metrics, calculated on sound files from Study IV (clear bird recordings with artifically added noise). It can be seen that PESQ scores are not consistently related to the quality of these sounds. In partic- 


\section{CONCLUSIONS}
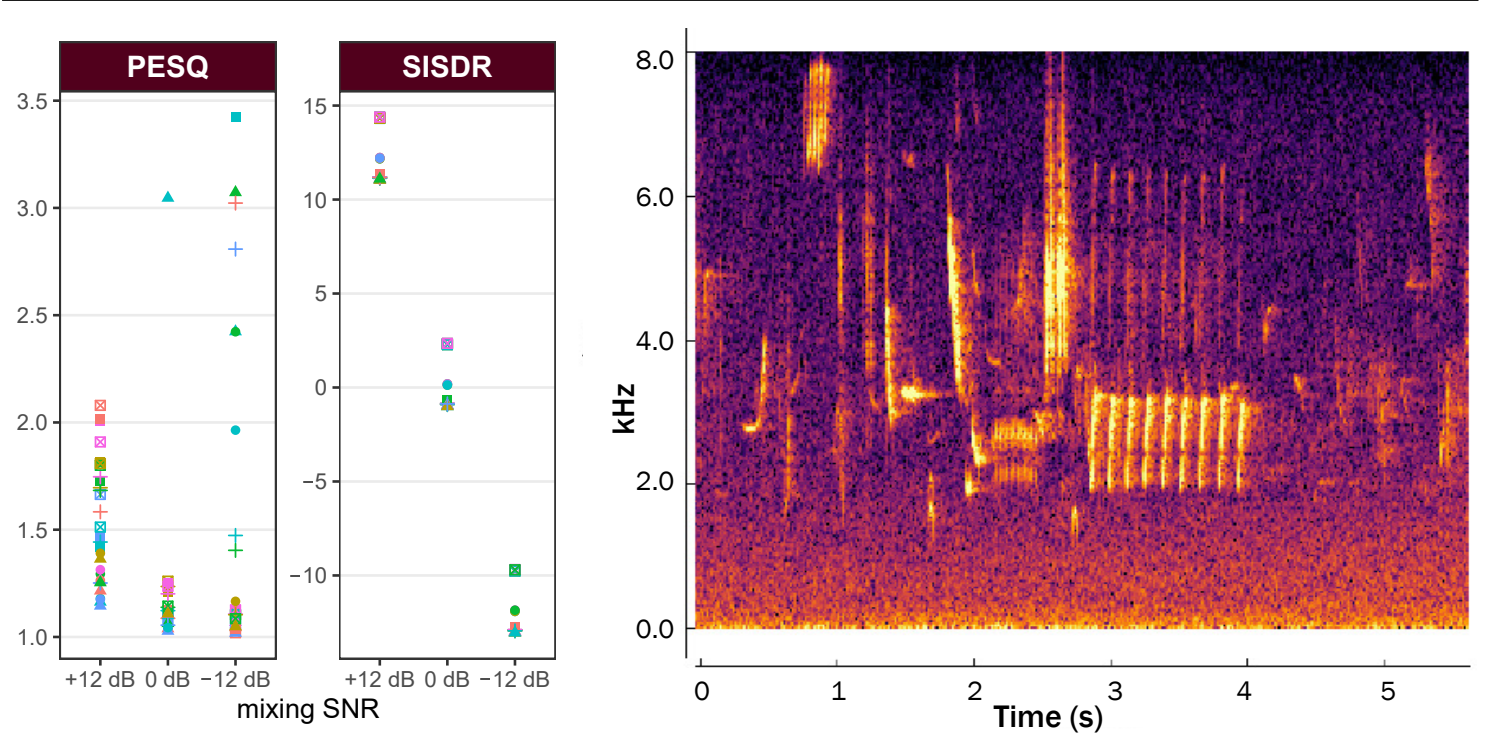

Figure 1: Left: PESQ and SISDR quality metrics of 90 files, obtained by mixing 6 bird recordings from xeno-canto (different colors) with 5 different noises (different shapes) at different SNR. Right: spectrogram showing part of a noisy file that has a PESQ of less than 1.5 .

ular, in low-SNR situations the scores seem to fluctuate wildly, without any relation to the strength or type of the signal. For comparison, the figure also shows scale-invariant signal-to-distortion ratio (SISDR, Roux et al. (2019)), which mostly matches the original mixing SNR. Even for loud and clear signals in this data, PESQ is reporting scores in the 1-2 range, indicating very low quality (Figure 1, right). Similar results can be seen in Brown et al. (2018), where composite scores, combined from PESQ and other speech metrics, have been used to measure bioacoustic sound quality. Possibly a more fruitful approach for bioacoustics could be to measure call similarity based on experimentally determined perception mechanisms (Bregman et al., 2016) or by distance in data-driven projection spaces (Sainburg et al., 2020).

\subsection{Spectrogram normalisation}

One source of variation that complicates spectrogram-based recognition is call loudness. More precisely, variable distance from the recorder, sound pressure at the source, and environmental attenuation contribute to create a large variation in the received signals; this affects not only the strength of the spectrogram patterns, but also the presence of harmonics and other call features (Priyadarshani et al., 2018). Furthermore, if we treat each column of the spectrogram as a windowed periodogram $\hat{I}(f)$ estimating the underlying spectral density $I(f)$, its sampling vari- 
ance $\operatorname{Var}(\hat{I}(f))$ also depends on these factors, and can be large - it is asymptotically equivalent to $I^{2}(f)$ (Priestley, 1982). Thus, spectrograms of the same call can appear very different visually, which likely impedes the recognition by CNNs and related methods.

To illustrate, we show the variation seen in recordings of a single morepork call, from the data in Priyadarshani et al. (2018). Two sets of clips were selected: 20 recordings, captured at a fixed distance from the source, and 20 recordings captured at distances of 20-120 m. We aligned the spectrograms in time, and for each time-frequency bin (i.e. pixel) calculated the sample variance of intensities at that pixel over the set. The unequal-distance group reveals a considerable variation in most parts of the call, especially higher harmonics, which are lost in the more distant recordings (Figure 2).

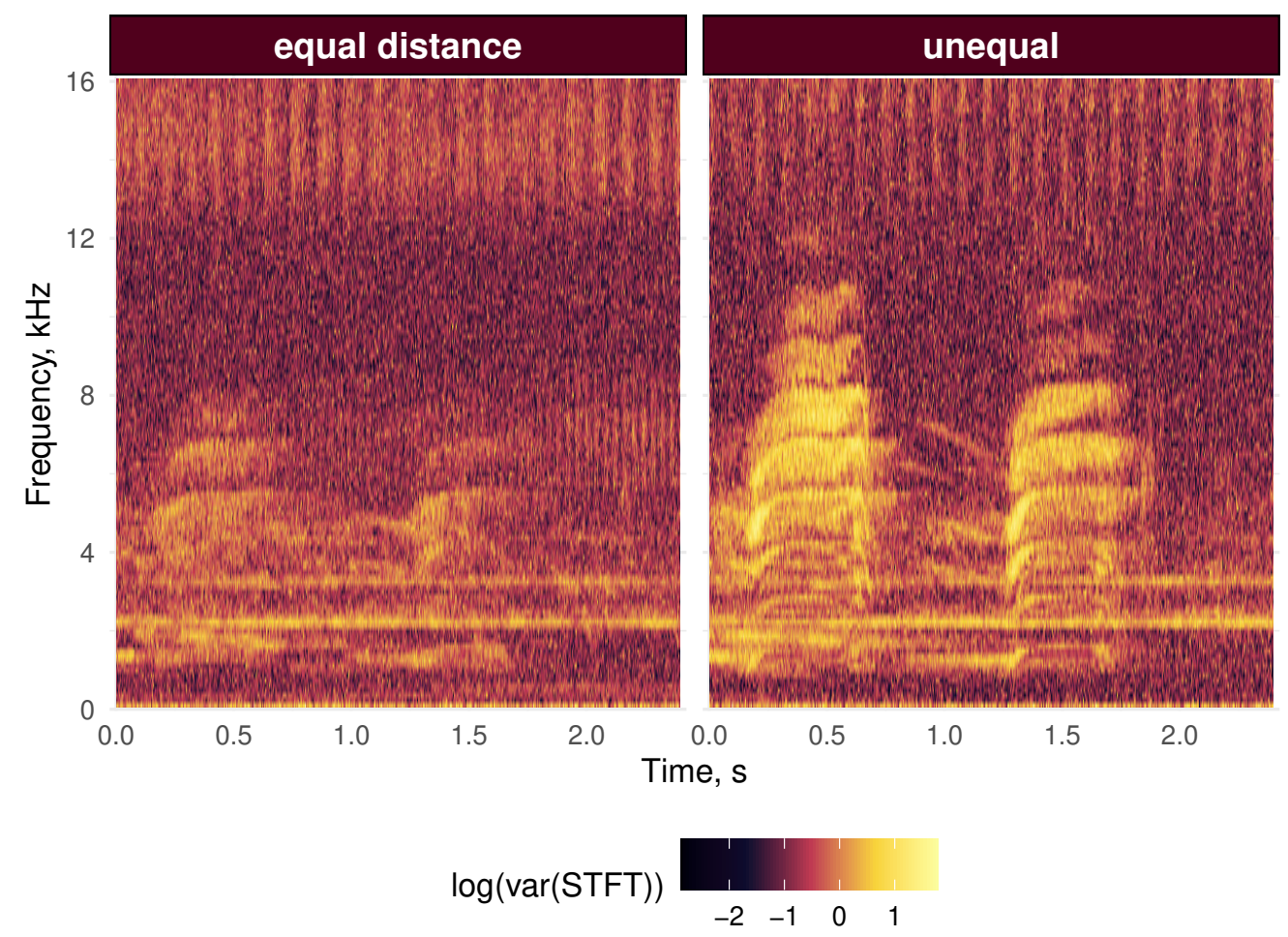

Figure 2: "Spectrograms" showing the variance of each pixel over two sets of spectrograms: 20 spectrograms from sounds recorded at equal distance to the source (left), and $20 \mathrm{spec}-$ trograms covering a range of distances (right). The source in all examples is playback of a morepork (Ninox novaeseelandiae) trill-type call.

While such mappings can certainly be learned with enough data, the task could be made simpler by appropriately standardising the spectrograms before analysis. For example, log-transforming the spectrogram magnitudes would map multiplicative changes to additive, and stabilise their sampling variance. Such scale changes 
in pre-processing have been shown to make a slight difference on classification performance in Knight et al. (2019) and Merchan et al. (2020). Lostanlen et al. (2019) showed that smoothing of the spectrogram over longer periods is beneficial. However, the adjustments presented in these studies were general, not adaptive to the loudness of each call. The methods developed in this thesis produce estimates of signal strength for each event (Studies II and III, Chapters 3-4) and of noise strength in short windows (Study IV, Chapter 5). Using these estimates, highly adaptive, short-term adjustments could be devised, to aid both CNNs and humans viewing the spectrograms. Simple metrics, such as the pixel variance used in Figure 2 , could be used to capture the loudness effects directly, without requiring full classification experiments.

\subsection{Extensions to different inference frameworks}

The methods presented in this thesis are not bound to a particular inference model. While we evaluate and analyse them mostly assuming inference by SCR, similar performance goals are also shared by other frameworks, as discussed in Chapter 1. Automatic characterisation of calls, or occupancy models that use individual call counts, would benefit from increased timestamp accuracy and fewer false positives obtained with the methods proposed here. With minor modifications, our detection and denoising algorithms can be converted to online forms, in the sense that segments at time $t$ can be detected without requiring many observations arriving after $t$. Combined with their computational simplicity, this makes our tools useful for creating real-time, low-latency detection systems as well.

However, minimal occupancy models that only use presence-absence do not require such performance properties, and therefore may benefit less from the proposed methods. We highlighted the complexity of evaluating improvements in the occupancy framework in Study I (Chapter 2). For these models, alternative statistical formulation of sound processing may lead to better tools.

Specifically, the presence-absence problem directly corresponds to the composite hypothesis setup (Chapter 1 ), with $H_{0}$ of target absence in a tested site and visit, and $H_{1}$ of presence. Tools that accurately distinguish between these would improve the subsequent analysis. However, connecting the call-level detection models to site-level properties is complicated under either of these hypotheses. One reason is that birds and mammals produce calls in complex sequences that have temporal structure and also depend on interactions between individuals (Miller et al., 2004). In other cases, marking separate calls may be difficult, but broad conclusions about 
relative vocal activity can still be made, such as when measuring seabird colony sizes (Oppel et al., 2014). New summary features could be designed to capture longer activity units of the target animal, possibly using acoustic indices, long-term spectrograms (Towsey et al., 2018), hidden Markov models for automatic description of call sequences (Bartcus et al., 2015), or unsupervised clustering of calls to individuals. Such features could give rise to new, finely detailed and statistically grounded models for inference at the site level.

\section{Bibliography}

Bartcus, M., Chamroukhi, F. and Glotin, H. (2015) Hierarchical Dirichlet Process Hidden Markov Model for unsupervised bioacoustic analysis. In 2015 International Joint Conference on Neural Networks (IJCNN). IEEE.

Bregman, M. R., Patel, A. D. and Gentner, T. Q. (2016) Songbirds use spectral shape, not pitch, for sound pattern recognition. Proceedings of the National Academy of Sciences, 113, 1666-1671.

Brown, A., Garg, S. and Montgomery, J. (2018) Automatic and efficient denoising of bioacoustics recordings using MMSE STSA. IEEE Access, 6, 5010-5022.

Cho, H. (2016) Change-point detection in panel data via double CUSUM statistic. Electronic Journal of Statistics, 10.

Jirak, M. (2015) Uniform change point tests in high dimension. The Annals of Statistics, 43.

Knight, E. C., Hernandez, S. P., Bayne, E. M., Bulitko, V. and Tucker, B. V. (2019) Preprocessing spectrogram parameters improve the accuracy of bioacoustic classification using convolutional neural networks. Bioacoustics, 29, 337-355.

Lostanlen, V., Salamon, J., Cartwright, M., McFee, B., Farnsworth, A., Kelling, S. and Bello, J. P. (2019) Per-channel energy normalization: Why and how. IEEE Signal Processing Letters, 26, 39-43.

Merchan, F., Guerra, A., Poveda, H., Guzmán, H. M. and Sanchez-Galan, J. E. (2020) Bioacoustic classification of Antillean manatee vocalization spectrograms using deep convolutional neural networks. Applied Sciences, 10, 3286.

Miller, P., Shapiro, A., Tyack, P. and Solow, A. (2004) Call-type matching in vocal exchanges of free-ranging resident killer whales, Orcinus orca. Animal Behaviour, 67, 1099-1107.

Oppel, S., Hervias, S., Oliveira, N., Pipa, T., Silva, C., Geraldes, P., Goh, M., Immler, E. and McKown, M. (2014) Estimating population size of a nocturnal burrow-nesting seabird using acoustic monitoring and habitat mapping. Nature Conservation, 7, 1-13.

Priestley, M. B. (1982) Spectral Analysis and Time Series, chap. 6. Academic Press. 
Priyadarshani, N., Castro, I. and Marsland, S. (2018) The impact of environmental factors in birdsong acquisition using automated recorders. Ecology and Evolution, 8, 5016-5033.

Rix, A., Beerends, J., Hollier, M. and Hekstra, A. (2001) Perceptual evaluation of speech quality (PESQ)-a new method for speech quality assessment of telephone networks and codecs. In 2001 IEEE International Conference on Acoustics, Speech, and Signal Processing. Proceedings (Cat. No.01CH37221). IEEE.

Roux, J. L., Wisdom, S., Erdogan, H. and Hershey, J. R. (2019) SDR - half-baked or well done? In ICASSP 2019 - 2019 IEEE International Conference on Acoustics, Speech and Signal Processing (ICASSP). IEEE.

Sainburg, T., Thielk, M. and Gentner, T. Q. (2020) Finding, visualizing, and quantifying latent structure across diverse animal vocal repertoires. PLOS Computational Biology, 16, e1008228.

Towsey, M., Znidersic, E., Broken-Brow, J., Indraswari, K., Watson, D. M., Phillips, Y., Truskinger, A. and Roe, P. (2018) Long-duration, false-colour spectrograms for detecting species in large audio data-sets. Journal of Ecoacoustics, 2, 1-1.

Vickers, W., Milner, B., Risch, D. and Lee, R. (2021) Robust North Atlantic right whale detection using deep learning models for denoising. The Journal of the Acoustical Society of America, 149, 3797-3812.

Zhang, N. R., Siegmund, D. O., Ji, H. and Li, J. Z. (2010) Detecting simultaneous changepoints in multiple sequences. Biometrika, 97, 631-645. 